\title{
Oak Ridge National Laboratory Annual Progress Report for the Power Electronics and Electric Machinery Program
}

Prepared by:

Oak Ridge National Laboratory

Mitch Olszewski, Program Manager

Submitted to:

Energy Efficiency and Renewable Energy FreedomCAR and Vehicle Technologies Vehicle Systems Team

Susan A. Rogers, Technology Development Manager

November 2005 
OAK RIDGE

NATIONAL LABORATORY

MANAGED BY UT-BATTELLE

FOR THE DEPARTMENT OF ENERGY

\section{Oak Ridge National Laboratory Annual Progress Report for the Power Electronics and Electric Machinery Program}

Mitch Olszewski, Program Manager

November 2005

Prepared by the

OAK RIDGE NATIONAL LABORATORY

Oak Ridge, Tennessee 37831 managed by

UT-BATTELLE, LLC

for the

U.S. DEPARTMENT OF ENERGY

Under contract DE-AC05-00OR22725

UT-BATTELLE

ORNL-27 (4-00) 


\section{Contents}

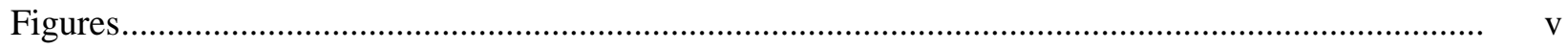

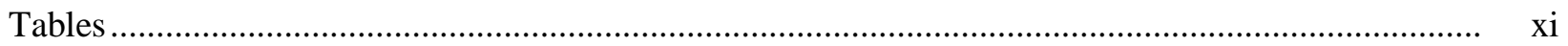

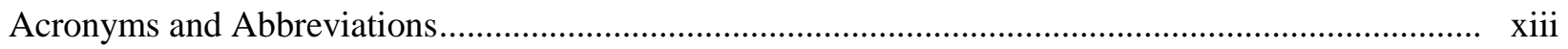

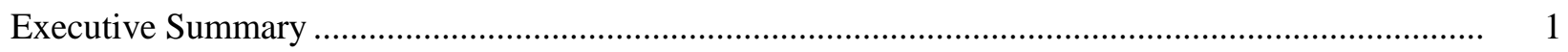

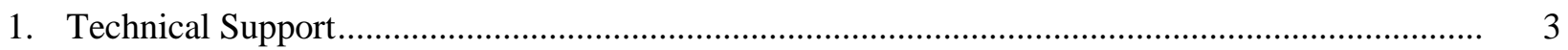

1.1 Power Electronics Crosscut Analysis ................................................................................... 3

1.2 Development of a Novel Bi-Directional Isolated Multiple-Input dc-dc Converter ................. 6

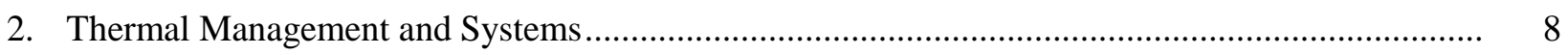

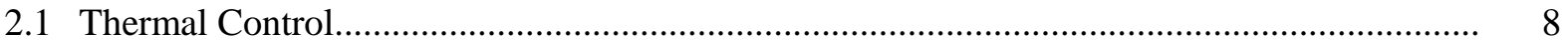

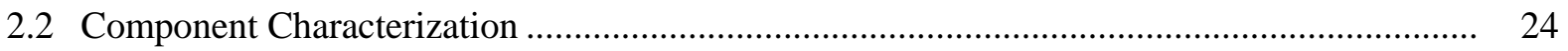

2.3 Benchmarking of Competitive Technologies ....................................................................... 34

3. Electric Machinery Research and Technology Development................................................. 45

3.1 Flux Weakening and CPSR Enhancement/Techniques ..................................................... 45

3.2 Electric Motor Research and Development ....................................................................... 93

3.3 Advanced Traction Motor Development (RFP) …............................................................. 105

4. Power Electronics Research and Technology Development .................................................. 108

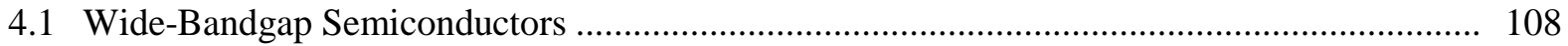

4.2 Integrated dc/dc Converter for Multi-Voltage Bus Systems .............................................. 128

4.3 Integrated Inverter for HEVs and Fuel Cell Powered Vehicles ............................................. 140

4.4 Z-Source Power Converter for Fuel Cell-Powered Vehicles ................................................ 154

4.5 dc-dc Converter for Fuel Cell and Hybrid Vehicle............................................................... 163

4.6 Semikron Automotive Integrated Power Module Testing ................................................. 171 



\section{Figures}

\subsection{Thermal Control}

1. Floating loop diagram (a); floating loop bench top apparatus with pump (b) ......................... 10

2. Revised floating loop attached to full-size sedan AC system in a cabinet ................................. 11

3. Floating loop/AC schematic with revisions ................................................................. 12

4. Conventional die mounting of inverter........................................................................... 12

5. Arrow indicates the conventional heat flux path of the conventional die-mounting inverter ....... 13

6. ORNL's cascaded die mounting concept ...................................................................... 13

7. Copper foam is used as an example for cascaded die mounting ............................................... 14

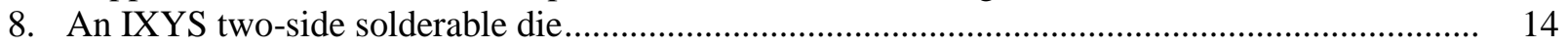

9. A two-side solderable die obtained from Rockwell Scientific .................................................. 14

10. (a) Prototype of copper-foam cascade die mounting, (b) two-phase cooling occurs

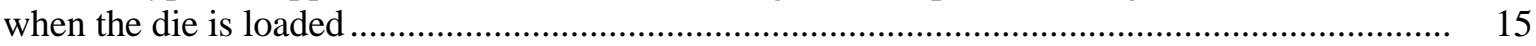

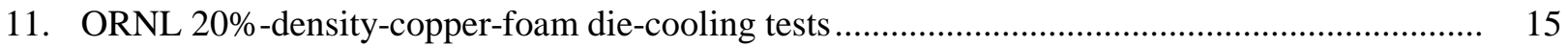

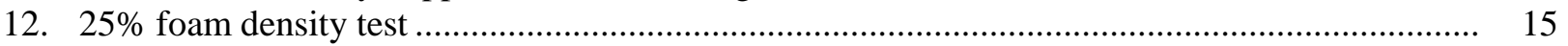

13. Example of an ORNL directly cooled film capacitor....................................................... 16

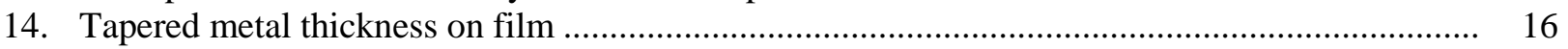

15. Photograph of Array B-2.4-MHz actuators ................................................................... 17

16. A schematic of the closed-loop WEG micro-jet array impingement cooling system for the Semikron inverter

17. ISR spray cooling system for the Semikron inverter....................................................... 18

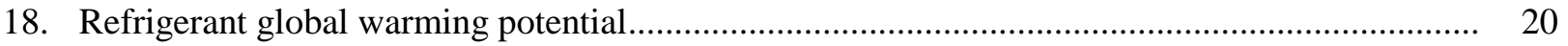

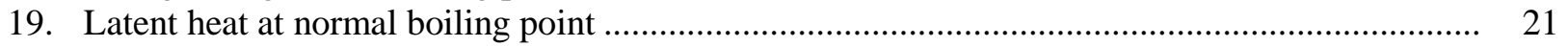

\subsection{Component Characterization}

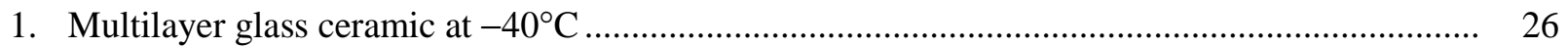

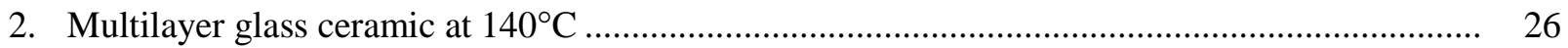

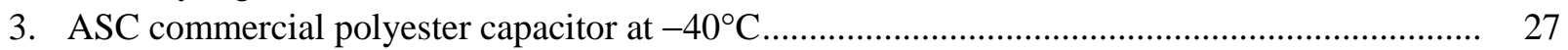

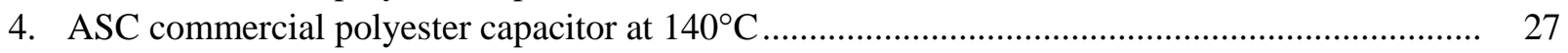

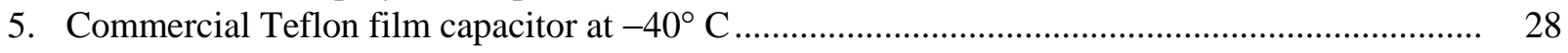

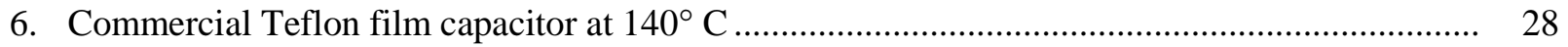

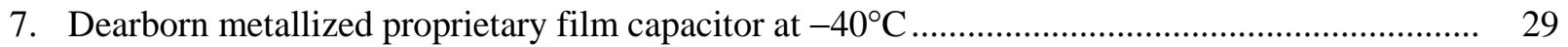

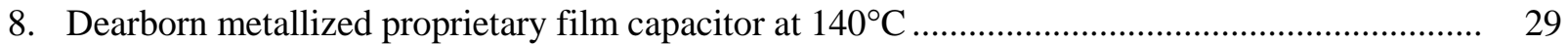

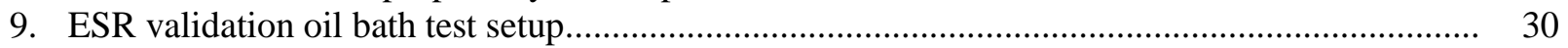

10. Walker Scientific AMH-40 Automatic Hysteresisgraph...................................................... 31

11. Ames 2205 bonded magnet demagnetization curves ........................................................... 32

12. Ames 60\% MPQ-O and 40\% PPS bonded magnet demagnetization curves ............................ 32

13. Argonne sintered magnet hysteresis graph..........................................................................

\subsection{Benchmarking of Competitive Technologies}

1. Test cell design, configuration, and actual layout ............................................................ 36

2. Upper-level flow diagram of Prius controller system ...................................................... 39

3. Voltage boost converter components and simplified schematic .......................................... 40

4. Extrapolation of generator back-EMF data for generator speeds of up to $10,000 \mathrm{rpm} \ldots \ldots \ldots \ldots \ldots . . . \ldots$

5. Drive cycle data from ANL showing voltage boost converter response .................................. 41

6. Drive cycle data set showing voltage boost at high speeds (ANL/ORNL data) ......................... 42 
7. Prius locked-rotor data showing torque vs rotor position

8. Prius controller/inverter system operating an IPM-reluctance motor with brushless field excitation.

\subsection{Field Weakening and CPSR Enhancement Techniques}

1. Field weakening and CPSR enhancement techniques........................................................ 49

2. Configuration of a Z-source inverter-driven FCV ….......................................................... 50

3. A configuration of an ICE-powered HEV driven by a Z-source inverter ................................. 51

4. Axial-gap consequent pole generator weakened and strengthened by external coil .................... 53

5. High-strength (magnetic) undiffused brushless axial-gap PM motor ...................................... 54

6. Variable flux PM motor-memory motor......................................................................... 55

7. Using the number of turns per slot to control CPSR ........................................................ 56

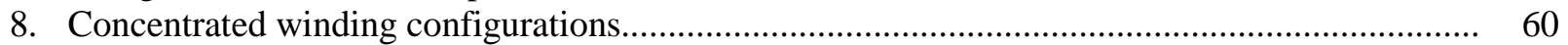

9. Basic repeating unit of the 6-kW SPM motor with fractional-slot concentrated windings ........... 61

10. Configuration of the ORNL model of a $6 \mathrm{~kW}$ fractional-slot PMSM motor made with concentrated windings.

11. One phase of the coil winding configuration for the ORNL model of a $6 \mathrm{~kW}$ fractional-slot PMSM motor with concentrated windings

12. Peak back-emf per phase of $6 \mathrm{~kW}$ motor at $800 \mathrm{rpm}$

13. Harmonic content in back-emf of $6 \mathrm{~kW}$ motor at $800 \mathrm{rpm}$

14. Per-phase equivalent circuit of $6 \mathrm{~kW}$ motor.

15. Relative permeance

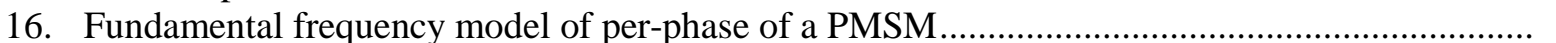

17. DMIC inverter topology

18. Per-phase fundamental frequency phasor model for constant power mode

19. RMS motor current vs. power output during high-speed operation of Motor 1 with 146.1-V and 203.7-V de supply voltages, when driven by CPA or by DMIC.

20. RMS motor current vs. power output during high-speed operation of Motor 1 and Motor 2 with 203.7 V dc supply

21. Per-phase model used to calculate average and rms device currents

22. Efficiency difference comparison of Motor 2 (with $208 \mathrm{~V} \mathrm{dc}$ supply voltage) minus Motor 1 (with $151 \mathrm{~V}$ dc supply voltage) when driven by CPA over the full torque speed envelope

23. Comparison of efficiency difference when Motor $1 \mathrm{dc}$ supply voltage is raised from 151 to $208 \mathrm{~V}$

24. Comparison of efficiency difference when Motor 1 is driven by DMIC and by CPA using inverter-grade thyristors from a $151-\mathrm{V}$ dc supply voltage

25. Efficiency difference between inverter-and converter-grade SCRs for Motor 1 driven by DMIC from a $151-\mathrm{V}$ dc supply voltage.

26. Standard RMS phasor diagram of a PM motor with flux weakening

27. Inset surface-mounted PM motors

28. V-shaped interior PM motors (type 4)

29. U-shaped interior PM motors (type 6)

30. Amount of PM material in terms of cross-sectional surface area as a function of $\beta \mathrm{M}$ or yl_1 for each of the three rotor configurations

31. Relationship between magnet pitch, $\beta \mathrm{M}$, speed, and percentage of reluctance torque for the inset PM motor.....

32. Magnet torque and total shaft torque of a four-pole inset reluctance-assisted PM motor for a range of magnet fractions. 


\subsection{Electric Motor Research and Development}

1. A sample RIPM-BFE prototype machine............................................................................. 94

2. RIPM-BFE rotor lamination............................................................................................. 95

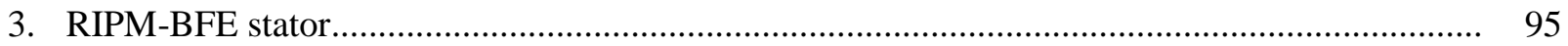

4. RIPM-BFE motor excitation coil ............................................................................... 96

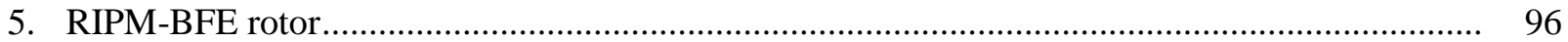

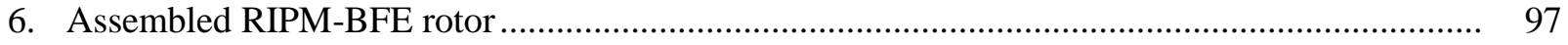

7. RIPM-BFE motor housing with excitation coil and spray ring ................................................. 97

8. Air-gap flux density distribution of RIPM-BFE 2.5-in. core-length motor. ............................... 98

9. Air-gap flux density distribution of THS II 3.3-in. core-length motor.......................................... 98

10. Machine design with side PMs to produce a strong fixed PM machine without field excitation.

11. RIPM-BFE locked-rotor torque vs rotor angular position without field enhancement/weakening .................................................................................... 99

12. RIPM-BFE locked-rotor torque vs rotor angular position with $5 \mathrm{~A}$ of field enhancement........... 100

13. Comparison of PM torque components and peak-torque components between a 2.5-in. ORNL machine and a 3.3-in. THSII machine ............................................................ 100

14. Corona initiating voltage test setup ................................................................................... 101

15. Clean trace without corona from 0 to $9000 \mathrm{Vrms}$ with silicone-sealed lead end .......................... 102

16. Corona initiating at 1638 Vrms for phase-to-phase and phase-to-ground test .............................. 102

\subsection{Advanced Traction Motor Development (RFP)}

1. Element mesh for Prius geometry and flux distribution.............................................................. 106

2. Predicted back-EMF waveform of the Toyota Prius traction drive motor ..................................... 107

\subsection{Wide-Bandgap Semiconductors}

1. Forward characteristics of SiC MOSFET at different temperatures ........................................... 109

2. On-resistance of SiC MOSFET at different temperatures.......................................................... 110

3. The gate and switching waveforms of the SiC MOSFET ………………………………........ 110

4. Energy loss plot of SiC MOSFET ............................................................................. 111

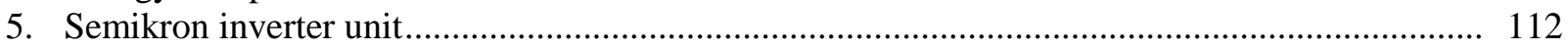

6. 75-A Schottky diodes developed by Cree …………………………………………………... 112

7. Measure (solid line) and simulated (dotted line) on-state waveforms of SiC Schottky diode at different temperatures........................................................................ 113

8. Measured (solid) and simulated (dotted) reverse-recovery waveforms of the SiC Schottky diode from Cree.

9. Comparison of peak-power output vs. efficiencies for hybrid and all-Si inverter inverter testing.

10. R-L load test efficiency curves for one of the load conditions.................................................. 115

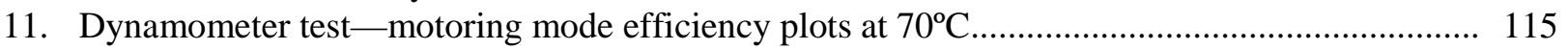

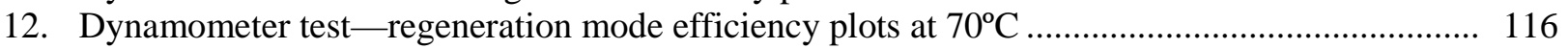

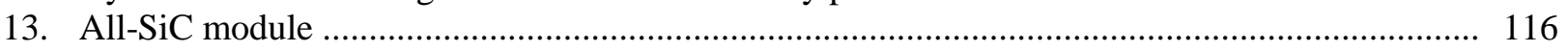

14. Rockwell SiC JFET measured (solid) and simulated (dotted) on-state waveforms at $250 \mathrm{C}$ for different gate voltages (Vgs ranging from $0 \mathrm{~V}$ to $-2.5 \mathrm{~V}$ ).

15. SiC JFET simulated (dashed) and measured (solid) turn-on waveforms at 25oC: (a) gate voltage, (b) drain voltage, and (c) drain current.

16. SiC JFET simulated (dashed) and measured (solid) turn-off waveforms at $25 \mathrm{oC}$ : (a) gate voltage, (b) drain voltage, and (c) drain current..................................................... 118

17. On-state validation of $\mathrm{SiC}$ diode at $25^{\circ} \mathrm{C}$ - measured (solid) and simulated (dashed) ................ 119

18. Reverse-recovery validation of $\mathrm{SiC}$ diode at $25^{\circ} \mathrm{C}$ - measured (solid) and simulated (dashed)...... 
19. Operating waveforms of SiC module for R-L load test.................................................... 120

20. Efficiencies comparison plot at $40 \mathrm{~Hz}$ operation for R-L load test ....................................... 120

21. Forward characteristics of IGBT and JFET in the $\mathrm{Si}$ and $\mathrm{SiC}$ modules .................................. 121

22. Forward characteristics of diodes in the $\mathrm{Si}$ and $\mathrm{SiC}$ modules .............................................. 121

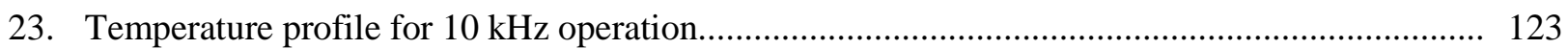

24. Comparison of static characteristics for Si IGBT and SiC JFET .......................................... 124

25. Temperature profile for diodes at different operating frequencies .......................................... 124

26. Temperature profile for switches at different operating frequencies ...................................... 125

27. Plot of temperature profile for power switches at different frequencies .................................. 125

\subsection{Integrated dc/dc Converter for Multi-Voltage Bus Systems}

1. A dc/dc converter interconnecting 14-V/42-V/high-voltage bus nets in HEVs and FCVs.......... 129

2. Schematic of the soft-switched bi-directional dc/dc converter ............................................... 130

3. Ideal transformer voltage and current waveforms illustrating power flow control between the $42-\mathrm{V}$ and high-voltage busses at $\mathrm{d}=1 / 3$.

4. Power v.s. phase shift angle at various levels of VHV $(n=8, L s=0.16 \mu \mathrm{H}$, $\mathrm{fsw}=40 \mathrm{kHz}, \mathrm{V} 42 \mathrm{v}=42 \mathrm{~V}$ ).....

5. Simulation results showing power is transferred from the low-voltage busses to the high-voltage net.

6. Simulation results illustrating power is transferred from the high-voltage net to the low-voltage busses

7. Photo of the prototype

8. Typical testing waveforms for $42-\mathrm{V}$ to $14-\mathrm{V}$ and high-voltage power transfer ...

9. Typical testing waveforms for high-voltage to $14-\mathrm{V}$ and $42-\mathrm{V}$ power transfer .

10. Typical testing waveforms for $14-\mathrm{V}$ to $42-\mathrm{V}$ and high-voltage power transfer.

11. Efficiency chart

12. Photo of the reduced-part count prototype.

13. Testing waveforms for $42-\mathrm{V}$ to $14-\mathrm{V}$ and high-voltage power transfer at $1.1 \mathrm{~kW}$

14. Testing waveforms for $42-\mathrm{V}$ to $14-\mathrm{V}$ and high-voltage power transfer at $1.4 \mathrm{~kW}$

15. Testing waveforms for high-voltage to $42-\mathrm{V}$ and $14-\mathrm{V}$ power transfer at $0.54 \mathrm{~kW}$

\subsection{Integrated Inverter for HEVs and Fuel Cell Powered Vehicles}

1. Existing configuration employing two separate three-phase inverter drives for traction and compressor motors in HEVs

2. Possible two-phase motor drive configurations .....

3. Proposed integrated inverter for driving a three-phase traction motor and a two-phase compressor motor ...

4. Equivalent circuits showing (a) inverter phase legs as voltage sources and

(b) the zero-sequence circuit of the main motor as the current return path

of the two-phase motor.

5. Dual inverter prototype without the gate driver and control boards...................................... 146

6. Dual inverter prototype — complete assembly ............................................................. 147

7. Dual inverter prototype connected for testing ................................................................. 148

8. Three-phase main PM motor mounted on the motor test bed with a 100-HP dyne .................... 148

9. Two-phase PM motor mounted on a portable 5-HP eddy current dyne motor test bed ............... 149

10. Modification of the three-phase motor into a two-phase one .................................................... 149

11. The main motor is not running while two-phase motor loaded with $10 \mathrm{~N} \cdot \mathrm{m}$ at $505 \mathrm{rpm} .10 \mathrm{~ms} / \mathrm{div}$.

12. The main motor is not running while two-phase motor loaded with $7.5 \mathrm{~N} \cdot \mathrm{m}$ at $1000 \mathrm{rpm} .5 \mathrm{~ms} / \mathrm{div}$. 
13. The two-phase motor is not running while the main motor is loaded with $40 \mathrm{~N} \cdot \mathrm{m}$ at $500 \mathrm{rpm} .10 \mathrm{~ms} / \mathrm{div}$

14. The two-phase motor is not running while the main motor loaded with $43 \mathrm{~N} \cdot \mathrm{m}$ at $1852 \mathrm{rpm} .2 \mathrm{~ms} / \mathrm{div}$

15. The main motor is loaded with $40 \mathrm{~N} \cdot \mathrm{m}$ at $750 \mathrm{rpm}$, and the two-phase motor is loaded with $12 \mathrm{~N} \cdot \mathrm{m}$ at $505 \mathrm{rpm} .10 \mathrm{~ms} / \mathrm{div}$

16. The main motor is loaded with $40 \mathrm{~N} \cdot \mathrm{m}$ at $1000 \mathrm{rpm}$, and the two-phase motor is loaded with $12 \mathrm{~N} \cdot \mathrm{m}$ at $800 \mathrm{rpm} .10 \mathrm{~ms} / \mathrm{div}$

4.4 Z-Source Power Converter for Fuel Cell-Powered Vehicles

1. System configuration using conventional PWM inverter

2. System configuration using $\mathrm{dc} / \mathrm{dc}$ boost $+\mathrm{PWM}$ inverter.

3. System configuration using the $\mathrm{Z}$ source inverter .............................................................. 156

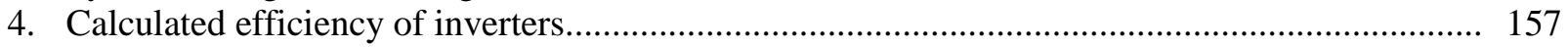

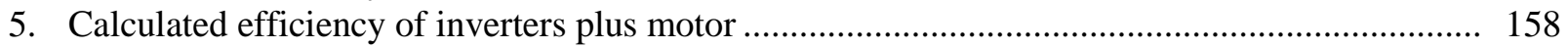

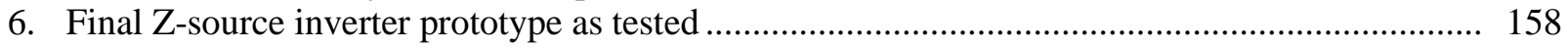

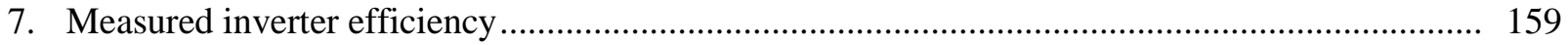

\section{5 dc-dc Converter for Fuel Cell and Hybrid Vehicle}

1. The novel interleaved dc-dc converter topology .....

2. A segmented preliminary $2.5-\mathrm{kW}$ dc-dc power module.................................................... 165

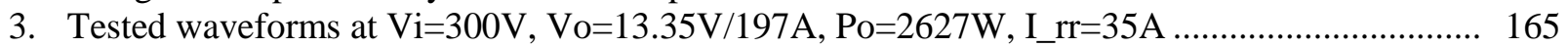

4. Efficiency mapping of the segmented dc-dc power module ................................................... 165

5. A 5-kW full functional dc-dc power module - alpha design ................................................. 166

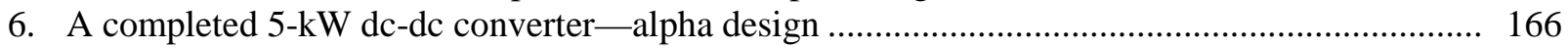

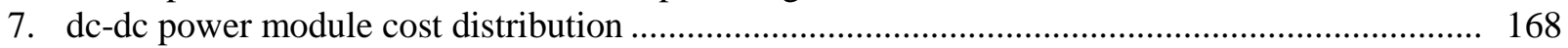

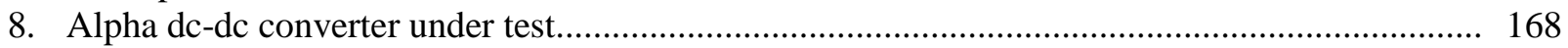

9. Alpha unit tested waveforms at $\mathrm{Vi}=300.6 \mathrm{~V}, \mathrm{Vo}=13.31 \mathrm{~V}, \mathrm{Po}=5.1 \mathrm{~kW}, \eta=93.3 \% \ldots \ldots \ldots \ldots \ldots \ldots . . . . . . . . .169$

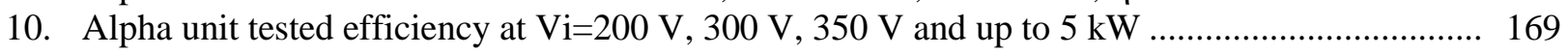

\subsection{Semikron Automotive Integrated Power Module Testing}

1. Top view of Semikron AIPM unit...

2. Control computer, power meter, power supply, and Semikron unit..................................... 172

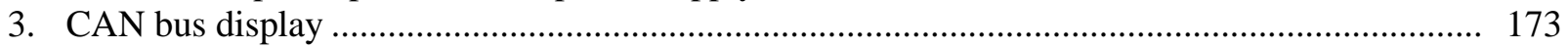

4. Inverter efficiency vs output power with $70^{\circ} \mathrm{C}$ coolant ................................................. 176

5. Power meter readings and current waveforms at end of continuous power test with $70^{\circ} \mathrm{C}$ coolant.

6. Power meter readings and current waveforms at end of peak power test with $70^{\circ} \mathrm{C}$ coolant....... 177

7. Inverter efficiency vs motor speed with $70^{\circ} \mathrm{C}$ coolant .................................................. 178

8. Semikron inverter efficiency vs speed at continuous power with $70^{\circ} \mathrm{C}$ coolant ........................ 180

9. Power meter readings and current waveforms during continuous power test on dyne with $70^{\circ} \mathrm{C}$ coolant. 



\section{Tables}

Page

\subsection{Thermal Control}

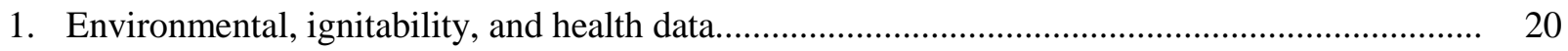

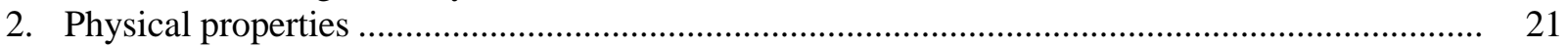

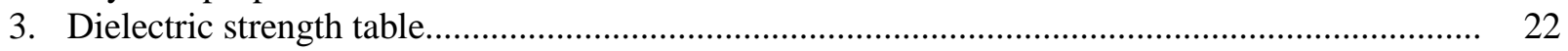

\subsection{Benchmarking of Competitive Technologies}

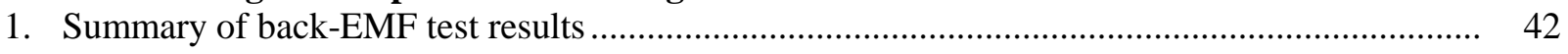

2. Summary of motor current and torque test results ...........................................................

\subsection{Field Weakening and CPSR Enhancement Techniques}

1. Stator dimensions and winding data for the 36-slot/30-pole SPM machine ........................... 61

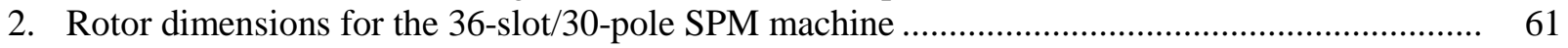

3. Calculated inductances for the 36-slot/30-pole SPM machine............................................. 61

4. Calculated current and magnet parameters for the 36-slot/30-pole SPM machine ..................... 62

5. Breakdown of material mass for the 36 -slot/30-pole SPM machine...................................... 62

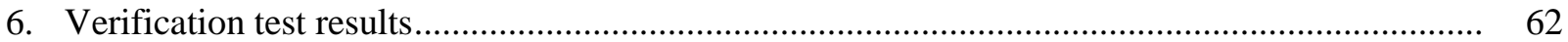

7. Comparison of $6 \mathrm{~kW}$ fractional-slot motor analyses ............................................................... 64

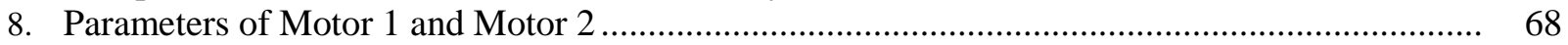

\subsection{Electric Motor Research and Development}

1. Summary of test results of corona

\subsection{Z-Source Power Converter for Fuel Cell-Powered Vehicles}

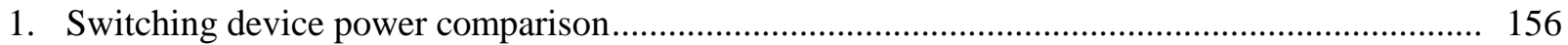

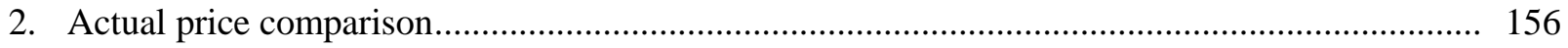

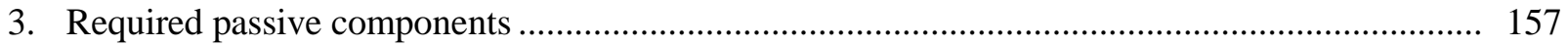

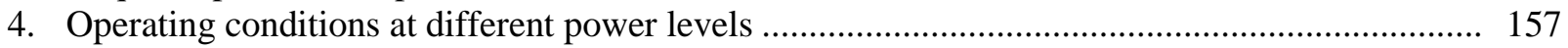

5. Comparison of the prototype and the FreedomCAR goals................................................... 160

\section{5 dc-dc Converter for Fuel Cell and Hybrid Vehicle}

1. Alpha dc-dc converter bill of material and cost estimation at $1000 \mathrm{~K}$ volume .......................... 167

2. dc-dc power module cost spreadsheet ............................................................................ 167

3. Up-to-date status summary from the alpha design ............................................................ 170

\subsection{Semikron Automotive Integrated Power Module Testing}

1. Short-duration testing of Semikron inverter with 200-Vdc link............................................. 173

2. Short-duration testing of Semikron inverter with $250-$ Vdc link ............................................... 173

3. Short-duration testing of Semikron inverter with 400- and 450-Vdc link ................................. 174

4. Short-duration testing of Semikron inverter with 325-Vdc link............................................ 175

5. Continuous power testing of Semikron inverter with 325-Vdc link ....................................... 177

6. Testing of Semikron inverter at greater than continuous power levels .................................... 177

7. Dynamometer testing of Semikron inverter—speed vs inverter efficiency ............................... 179

8. Specific power density summary ........................................................................... 181 



\section{Acronyms and Abbreviations}

\begin{tabular}{|c|c|}
\hline $\mathrm{AC}$ & air-conditioning \\
\hline $\mathrm{ac}$ & alternating current \\
\hline AIPM & automotive integrated power module \\
\hline ANL & Argonne National Laboratory \\
\hline BDCM & brushless direct current motor \\
\hline BFE & brushless field excitation \\
\hline CAN & controller area network \\
\hline CFC & chlorofluorocarbon \\
\hline $\mathrm{COP}$ & coefficient of performance \\
\hline $\mathrm{CPA}$ & conventional phase advance \\
\hline CPSR & constant power speed range \\
\hline CSI & current-source inverter \\
\hline DAC & data acquisition system \\
\hline d-axis & direct-axis \\
\hline DMIC & dual-mode inverter control \\
\hline DOE & U.S. Department of Energy \\
\hline DSP & digital signal processor \\
\hline EERE & Energy Efficiency and Renewable Energy \\
\hline emf & electromotive force \\
\hline EMI & electromagnetic interference \\
\hline ESR & equivalent series resistance \\
\hline $\mathrm{FC}$ & fuel cell \\
\hline FCV & fuel cell vehicle \\
\hline FEA & finite-element analysis \\
\hline FVCT & FreedomCAR and Vehicle Technologies \\
\hline $\mathrm{GaN}$ & gallium nitride \\
\hline GT & Georgia Institute of Technology \\
\hline GWP & global warming potential \\
\hline $\mathrm{HC}$ & hydrocarbon \\
\hline $\mathrm{HCFC}$ & hydrochlorofluorocarbon \\
\hline $\mathrm{HEV}$ & hybrid electric vehicle \\
\hline HFC & hydrofluorocarbon \\
\hline HFE & hydrofluoroether \\
\hline HSUPM & hybrid-secondary-uncluttered permanent magnet \\
\hline HVAC & heating, ventilating, and air-conditioning \\
\hline ICE & internal combustion engine \\
\hline IGBT & insulated gate bipolar transistor \\
\hline IM & induction motor \\
\hline IPM & integrated power module \\
\hline IPM & interior permanent magnet \\
\hline I-source & current-source \\
\hline ISR & Isothermal Systems Research \\
\hline JFET & junction field-effect transistor \\
\hline $\mathrm{JIC}$ & jet impingement cooling \\
\hline $\mathrm{mmf}$ & magneto-motive \\
\hline MOSFET & metal oxide semiconductor field-effect transistor \\
\hline MS & methylsiloxane \\
\hline MSU & Michigan State University \\
\hline
\end{tabular}


NASA

NTRC

ODP

OFCVTs

ORNL

PEEM

PEEMRC

PFC

PMDC

PMSM

PWM

q-axis

R\&D

$\mathrm{rad} / \mathrm{s}$

RFP

RIPM

rms

RSC

RTFC

SDPR

$\mathrm{Si}$

$\mathrm{SiC}$

SKAI

SMPM

SOC

SPM

THS II

toff

ton

UWM

VIBE

$\mathrm{V}$-source

VSI

WBG

WEG

ZSC

ZVS
National Aeronautics and Space Adminstration

National Transportation Research Center

ozone-depleting potential

Office of FreedomCAR and Vehicle Technologies

Oak Ridge National Laboratory

Power Electronics and Electric Machines

Power Electronics and Electric Machinery Research Center

perfluorocarbon

permanent magnet direct current

permanent magnet synchronous motor

pulse-width modulation

quadrature-axis

research and development

rotational speed

request for proposals

reluctance interior permanent magnet

root-mean-square

Rockwell Scientific Company

real time flux control

switching device power rating

silicon

silicon carbide

Semikron Advanced Integration

surface mounted permanent magnet

state-of-charge

surface-mounted PM motor

Toyota hybrid system (2004)

turn-off times

turn-on

University of Wisconsin, Madison

vibration-induced bubble ejection

voltage-source

voltage source inverter

wide bandgap

water-ethylene glycol

zero-sequence circuit

zero-voltage-switching 


\section{Executive Summary}

The U.S. Department of Energy (DOE) and the U.S. Council for Automotive Research (composed of automakers Ford, General Motors, and DaimlerChrysler) announced in January 2002 a new cooperative research effort. Known as FreedomCAR (derived from "Freedom" and "Cooperative Automotive Research"), it represents DOE's commitment to developing public/private partnerships to fund high-risk, high-payoff research into advanced automotive technologies. Efficient fuel cell technology, which uses hydrogen to power automobiles without air pollution, is a very promising pathway to achieve the ultimate vision. The new partnership replaces and builds upon the Partnership for a New Generation of Vehicles initiative that ran from 1993 through 2001.

The Vehicle Systems subprogram within the FreedomCAR and Vehicle Technologies Program provides support and guidance for many cutting-edge automotive and heavy truck technologies now under development.

Research is focused on understanding and improving the way the various new components of tomorrow's automobiles and heavy trucks will function as a unified system to improve fuel efficiency. This work also supports the development of advanced automotive accessories and the reduction of parasitic losses (e.g., aerodynamic drag, thermal management, friction and wear, and rolling resistance).

In supporting the development of hybrid propulsion systems, the Vehicle Systems subprogram has enabled the development of technologies that will significantly improve fuel economy, comply with projected emissions and safety regulations, and use fuels produced domestically.

The Vehicle Systems subprogram supports the efforts of the FreedomCAR and Fuel and the 21st Century Truck Partnerships through a three-phase approach intended to

- Identify overall propulsion and vehicle-related needs by analyzing programmatic goals and reviewing industry's recommendations and requirements, then develop the appropriate technical targets for systems, subsystems, and component research and development activities;

- Develop and validate individual subsystems and components, including electric motors, emission control devices, battery systems, power electronics, accessories, and devices to reduce parasitic losses; and

- Determine how well the components and subsystems work together in a vehicle environment or as a complete propulsion system and whether the efficiency and performance targets at the vehicle level have been achieved.

The research performed under the Vehicle Systems subprogram will help remove technical and cost barriers to enable technology for use in such advanced vehicles as hybrid and fuel-cell-powered automobiles that meet the goals of the FreedomCAR Program.

A key element in making hybrid electric vehicles practical is providing an affordable electric traction drive system. This will require attaining weight, volume, and cost targets for the power electronics and electrical machines subsystems of the traction drive system. Areas of development include

- Novel traction motor designs that result in increased power density and lower cost;

- Inverter technologies involving new topologies to achieve higher efficiency and the ability to accommodate higher-temperature environments;

- Converter concepts that employ means of reducing the component count and integrating functionality to decrease size, weight, and cost;

- More effective thermal control and packaging technologies; and

- Integrated motor/inverter concepts.

The Oak Ridge National Laboratory's (ORNL's) Power Electronics and Electric Machinery Research Center conducts fundamental research, evaluates hardware, and assists in the technical direction of the DOE Office of FreedomCAR and Vehicle Technologies Program, Power Electronics and Electric Machinery Program. In this role, ORNL serves on the FreedomCAR Electrical and Electronics Technical 
Team, evaluates proposals for DOE, and lends its technological expertise to the direction of projects and evaluation of developing technologies.

ORNL also executes specific projects for DOE. The following report discusses those projects carried out in FY 2004 and conveys highlights of their accomplishments. Numerous project reviews, technical reports, and papers have been published for these efforts, if the reader is interested in pursuing details of the work. 


\section{Technical Support}

\subsection{Power Electronics Crosscut Analysis}

Principal Investigator: Sam Nelson

Oak Ridge National Laboratory

National Transportation Research Center

2360 Cherahala Boulevard

Knoxville, TN 37932

Voice: 865-946-1327; Fax: 865-946-1262; E-mail: neslonsscjr@ornl.gov

DOE Technology Development Manager: Susan A. Rogers

Voice: 202-586-8997; Fax: 202-586-1600; E-mail: Susan.Rogers@ee.doe.gov

ORNL Program Manager: Mitch Olszewski

Voice: 865-946-1350; Fax: 865-946-1262; E-mail: olszewskim@ornl.gov

\section{Objectives}

The objective of the Energy Efficiency and Renewable Energy (EERE) Power Electronics Crosscut task is to organize and implement technology cross-cutting research tasks in power electronics. Potential areas of research will be identified that will be jointly funded by two or more offices within EERE leveraging PEEM research funds. Power electronics and electric machinery applications span many areas of research currently being funded by a number of agencies of the federal government.

\section{Approach}

The approach for this effort is to bring together Power Electronics and Electric Machines (PEEM) program managers and researchers from within FreedomCAR and Vehicle Technologies (FVCT) subprograms, other DOE programs and offices, government agencies, and industry partners who are interested in pursuing a common goal. The common goal is to improve the performance and reduce the cost, weight, and volume of PEEM components and systems. Strategic collaboration will be needed to identify key areas of expertise, gain insights for developing research goals and technical targets, and establish projects of mutual benefit.

To successfully complete the objectives of this task, several subtasks that define the overall approach to this task must be accomplished. They include

- identifying potential areas of focus

- $\quad$ selecting research and development $(\mathrm{R} \& \mathrm{D})$ projects

- identifying funding partners

- identifying a potential research organization to perform the $R \& D$

- establishing an agreement

- monitoring the R\&D

\section{Major Accomplishments}

During FY 2005, the Advanced Power Electronics and Electric Machines technology development manager funded the tasks required to forge partnerships within EERE, and the FVCT program manager funded the R\&D activity. A crosscut task was identified in silicon carbide (SiC) research to prepare a comprehensive study and report on the state-of-the-art technology in $\mathrm{SiC}$ power electronics and to assess $\mathrm{R} \& \mathrm{D}$ needs so that full advantage may be taken of this new material for power electronics devices. 
A workshop was held June 8, 2005, with a National Aeronautics and Space Adminstration (NASA) representative to present an overview of power research and technology at NASA's Glenn Research Center.

\section{Technical Discussion}

Discussions and interactions occurred among FCVT, Solar Energy Technology, Hydrogen Fuel Cells and Infrastructure Technology, Distributed Energy and Energy Reliability, Building Technologies, and other offices about common areas of R\&D in PEEM. Several projects were considered, and the SiC assessment task was selected as a task to be funded in FY 2006.

A workshop was held on June 8, 2005, in Washington, D.C. James F. Soeder, Chief of the Advanced Electrical Systems Branch at NASA Glenn Research Center, made a presentation to DOE entitled "Exploration and Power Systems at NASA." The presentation provided an overview of power research and technology at Glenn Research Center. NASA's traditional space power systems (i.e., space station, satellites, and shuttle) are low-power units (i.e., less than $10 \mathrm{~kW}$ ) and operate at low voltages (i.e., less than $160 \mathrm{~V}$ ) in an environment with moderate temperatures. Traditional space power systems are created from one-of-a-kind components and have limited growth potential. NASA's power systems for exploration distribute high power (i.e., 10 to $1000 \mathrm{~kW}$ ) and operate in harsh environments with wide temperature ranges. These systems present many challenges, but they represent growth opportunities for power electronics. Some technology areas in power electronics might present opportunities for cooperation in R\&D between NASA and DOE. In addition to advanced inverter/converter R\&D, SiC components and high-temperature capacitors are areas of focus for both agencies.

\section{Conclusion}

Evaluations have shown a potential for duplication of effort among the various agencies with an interest in power electronics research. Collaboration among organizations with high-risk, long-range PEEM projects is highly desired to maximize the leverage gained from working with other organizations pursuing similar research goals and objectives. The funding of research tasks by multiple funding sources will result in more effective utilization of research funds. Power electronics crosscut analysis has an objective of reducing the duplication of effort by identifying PEEM R\&D tasks of mutual interest that can be jointly funded by organizations with common research interests.

\section{Future Direction}

The R\&D areas of focus for this task will be identified by the interaction between the program managers, PEEM researchers, and other interested parties. Discussions and interaction will continue with DOE programs and offices to identify and fund PEEM research that will advance knowledge and capability in power electronics technologies.

Some potential candidate tasks for power electronics crosscut research are

- Z-source inverters

- high-temperature packaging for electronics

- $\mathrm{dc} / \mathrm{dc}$ converters

- enhanced inverter control techniques

- electric machines with brushless field excitation

- permanent magnet R\&D

- compressed powdered material for stator cores

- finite element analysis characterization of motor parameters

- improved cooling techniques for inverters and motors

- high-dielectric-strength capacitors 
This list is not all-inclusive, and there are additional areas of PEEM R\&D that should receive consideration for future research funds.

\section{References}

1. R. L. Smith and R. J. Kevala, Power Electronics Crosscut Activities for EERE and OETD, Final Report, U.S. Department of Energy, Washington, D.C., September 30, 2004. 


\title{
1.2 Development of a Novel Bi-Directional Isolated Multiple-Input dc-dc Converter
}

\author{
Principal Investigator:Laura Marlino \\ Oak Ridge National Laboratory \\ National Transportation Research Center \\ 2360 Cherahala Boulevard \\ Knoxville, TN 37932 \\ Voice: 865-946-1245; Fax:865-946-1262; E-mail:marlinold@ornl.gov \\ DOE Technology Development Manager: Susan A. Rogers \\ Voice: 202-586-8997; Fax: 202-586-1600; E-mail: Susan.Rogers@ee.doe.gov \\ ORNL Program Manager: Mitch Olszewski \\ Voice: 865-946-1350; Fax: 865-946-1262; E-mail: olszewskim@ornl.gov
}

\section{$\underline{\text { Objectives }}$}

The objective is to develop a low-cost, lightweight, high-power-density bi-directional dc-dc converter to interface multilevel energy storage elements for fuel cell vehicles (FCVs) in order to optimize the power flow between the fuel cell and on board energy storage elements such as the battery and ultracapacitors. This topology will result in a system with an increased life cycle, optimized size and weight for fuel cell and energy storage elements, and improved vehicle system dynamic behavior and efficiency.

\section{Approach}

The proposed converter technology utilizes magnetic flux linkage to provide a controllable way to combine energy from different input sources, and the transformer makes the converter capable of connecting three ports: the battery, the ultracapacitor, and the load, with galvanic isolation between the load and sources. The converter can also be viewed as an extension of a single-input soft-switched bidirectional dc-dc converter, and so maintains the advantages of soft-switching technologies, along with requiring a minimum number of devices, and a simple control methodology.

\section{Major Accomplishments}

- Analyzed the operating principles of the proposed converter and verified them with simulation results.

- Derived the design guidelines of the converter for fuel cell vehicle applications.

- Developed the computer software to design and size the proposed converter.

- Constructed the 5-kW hardware prototype of the proposed dc-dc converter.

- Tested the hardware prototype of the proposed dc-dc converter and derived experimental results to verify the steady-state power flow control.

\section{Technical Discussion}

The proposed converter has the following advantages:

- Electrical isolation can be achieved naturally.

- The magnitude of dc input voltages may be low and similar or dissimilar.

- The dc sources can deliver power individually, simultaneously, and bi-directionally.

- The soft-switching technology is achievable in a wide operating range. 
- The number of devices is minimal, and control is simple.

- It is light in weight and has high power density.

The size of this converter is $12 \times 15 \times 7 \mathrm{in}$. The converter is operated at a switching frequency of $20 \mathrm{kHz}$ or higher. The transformer-turns ratio is $1: 12$. The current implementation cost of this converter is $\$ 1,950$. The power density of the prototype reaches $4 \mathrm{~W} /$ in. $^{3}$ (continuous).

\section{Conclusion}

A low-cost multiple-input dc-dc converter has been proposed, analyzed, designed, built, and tested. It uses only six switching devices, leading to significant cost savings and high power density. It requires no auxiliary circuits or complex control to achieve soft switching. Flexible power flow is obtained by controllable leakage inductance and a phase shift angle control scheme. The analysis, simulation, and experimental results confirmed the operating principles of the proposed converter.

\section{Future Direction}

- The power flow from the high-voltage to the low-voltage side needs to be experimentally verified.

- The power flow control scheme during transients-including start-up, acceleration, and deceleration-needs to be developed to optimize the operation of the energy storage elements.

- The weight and power density can be further improved by advanced power electronics technology.

\section{$\underline{\text { Publications }}$}

Danwei Liu and Hui Li, "A Novel Multiple-Input ZVS Bidirectional dc- dc Converter," to be presented at IECON 2005.

\section{Patents}

To be filed soon.

\section{References}

1. Yaow-Ming Chen, "Multiple-input dc/dc Converter Based on the Multiwinding Transformer for Renewable Energy Application," in IEEE Trans. on Industry Applications, 38(4), 1096-1103 (July/August 2002),

2. L. Solero, A. Lidozzi, and J.A. Pomilio, "Design of Multiple-Input Power Converter for Hybrid Vehicles,” pp. 1145-1151 in Proc. IEEE APEC’04, 2004.

3. Fang Z. Peng, Hui Li, Gui-jia Su, and J. Lawler, "A New ZVS Bi-directional dc-dc Converter for Fuel Cell and Battery Applications," IEEE Transaction on Power Electronics, 19(1), 54-65 (January 2004). 


\section{Thermal Management and Systems}

\subsection{Thermal Control}

Principal Investigator: John S. Hsu

Team Members: Curtis Ayers, Steven Campbell, Jim Conklin, Chester Coomer, Michael Jenkins, Kirk Lowe, Marshall McFee, Larry Seiber, Michael Starke, Randy Wiles

Oak Ridge National Laboratory

National Transportation Research Center

2360 Cherahala Boulevard

Knoxville, TN 37932

Voice: 865-946-1325; Fax:865-946-1262 ; E-mail: hsujs@ornl.gov

DOE Technology Development Manager: Susan A. Rogers

Voice: 202-586-8997; Fax: 202-586-1600; E-mail: Susan.Rogers@ee.doe.gov

ORNL Program Manager: Mitch Olszewski

Voice: 865-946-1350; Fax: 865-946-1262; E-mail: olszewskim@ornl.gov

\section{Objectives}

The goal of this effort is to prove the effectiveness of using thermal methods to reduce the size of the current $600-\mathrm{V}$ Semikron SKAI inverter by $50 \%$ of its current size in 2006, and then reduce it to one-third of the original size in 2008 without negatively impacting power density, cost, life expectancy, and performance of the entire system. This should result in a cost savings for the production of the overall system.

The objectives for FY 2005 included

- assessment of emerging two-phase cooling technologies,

- development of floating-loop technologies using high- or low-pressure refrigerants that can be operated independently without a compressor,

- development of methods of die mounting for better heat transfer and to reduce the inverter size

- develoopment of means of capacitor cooling,

- development of inverter/converter packaging techniques, and

- development of motor cooling techniques.

\section{Approach}

During FY 2005, Oak Ridge National Laboratory (ORNL) assessed three cooling techniques to determine if they could be beneficial for power electronics in hybrid electric vehicles (HEVs). The technologies were vibration-induced bubble ejection (VIBE) cooling from Innovative Fluidics/Georgia Institute of Technology (GT), a jet impingement system from Rockwell Scientific Company (RSC), and a spray cooling system from Isothermal Systems Research (ISR).

A floating-loop cooling system for automotive applications was developed, and a prototype system was incorporated into an automotive AC system. This system has the capability of using high- or lowpressure refrigerants to cool motors, insulated gate bipolar transistors (IGBTs), diodes, and capacitors independently of the compressor. 


\section{Major Accomplishments}

- The floating-loop concept for cooling inverters and motors of HEVs and electric vehicles was validated through tests.

- ORNL's novel silicon die mounting and cooling techniques were prototyped and evaluated. These innovations will allow for potential cost and size reductions in the inverter. The heat flux of the copper-foam die mounting structure cooled by R134A refrigerant reached $170 \mathrm{~W} / \mathrm{cm}^{2}$.

- The work on a new direct cooling method for film capacitors was begun. It offers the possibility of a significant reduction in capacitor size required.

- The interim report on the evaluation of emerging two-phase cooling technologies was completed.

- The coolant fluid study (including dielectric strength) was completed.

\section{$\underline{\text { Technical Discussion }}$}

\section{Floating Refrigerant Loop Based on R-134a Refrigerant Cooling of High-Heat-Flux Electronics}

The proof-of-concept work conducted on the floating loop is detailed in the report, Floating Refrigerant Loop Based on R-134a Refrigerant Cooling of High Heat Flux Electronics, ORNL/TM2005/223. The concept was successfully proved. This loop (based on R-134a) is integrated with a vehicle's existing AC condenser, which dissipates waste heat to the ambient air. Because the temperature requirements for power electronics and electric machines are not as low as that required for passenger compartment air, this adjoining loop can operate on the high-pressure side of the existing AC system. This arrangement also allows the floating loop to run without the compressor and only needs a small pump to move the liquid refrigerant. For the design to be viable, the loop must not adversely affect the existing system. The loop should also provide a high coefficient of performance (COP), a flat-temperature profile, and low-pressure drop.

\section{Proof-of-concept}

To demonstrate the refrigerant cooling concept for power electronics, three main project goals were set. The first step was to show that a significant heat load could be removed with no compressor, and the refrigerant could be moved through the system with a liquid pump or vapor blower, requiring minimal input power. This would prove that a low-pressure drop, high-temperature, two-phase coolant loop would remove sufficient heat by itself.

The second step was to attach a similar loop to an automotive AC system where the loop and AC system would share the condenser. The objective of the second goal was to prove that the loop could share the condenser without adversely affecting the inventory behavior or performance of the automotive AC. In a full hybrid setup, the loop would operate constantly. The second configuration would realistically only come into question if the loop was used on an assist motor where the internal combustion engine runs all the time, and the electric assist is more intermittent.

The last goal was to operate the floating loop and AC simultaneously. This test would be indicative of normal operation of the floating loop in a full hybrid configuration. The loop would be cooling a wasteheat load from the power electronic components, while the AC system satisfied the passengers' compartment air temperature requirements.

\section{Prototype results}

The first goal was met with a bench-top setup consisting of a variable-speed pump, heat source, and condenser (Figures 1a and 1b). This cooling loop removed more than $2.1 \mathrm{~kW}$ of heat load with minimal input power. A vapor blower and pump were both tried with input power of 210 and $48 \mathrm{~W}$, respectively. 


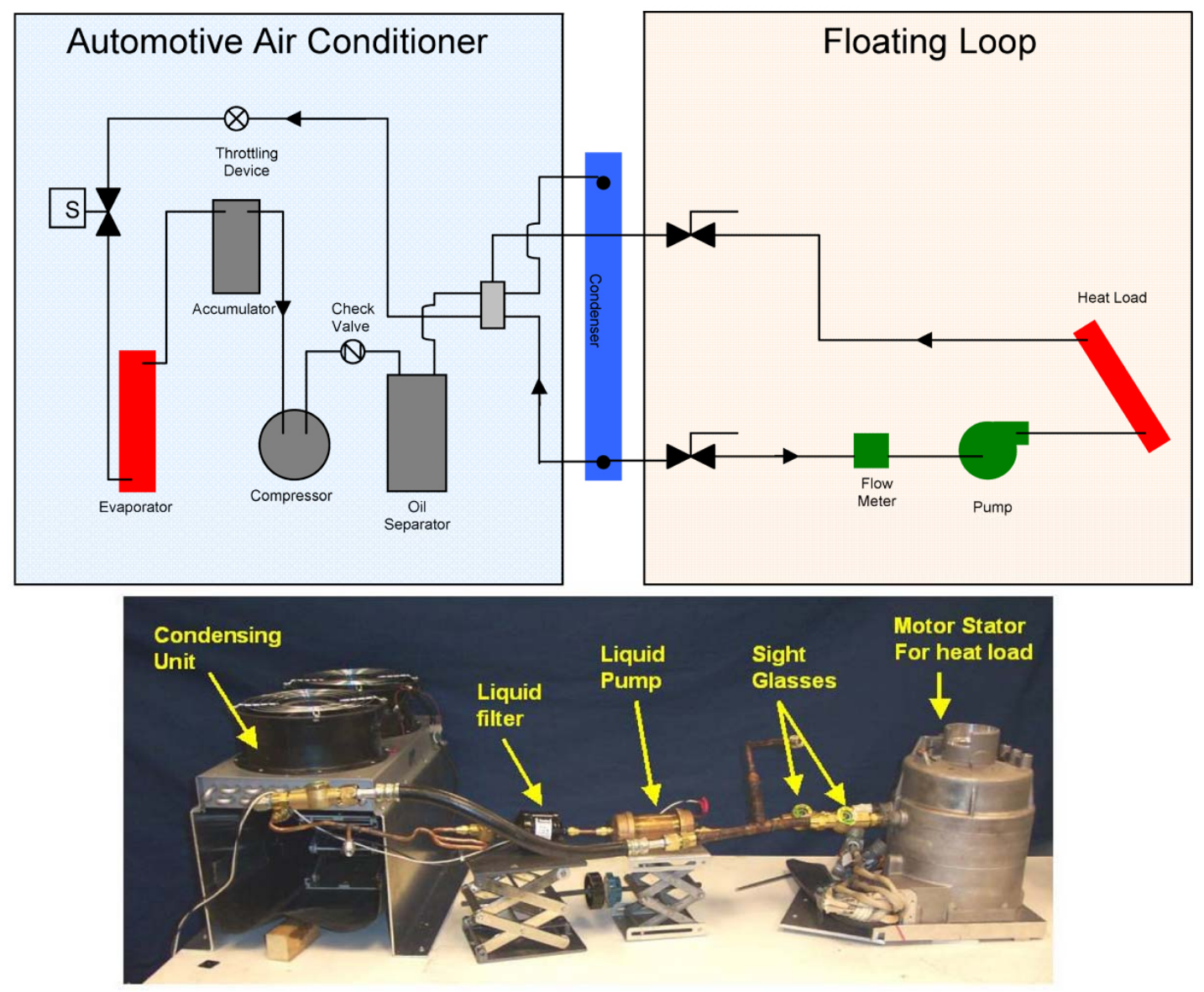

Figure 1. Floating loop diagram (a); floating loop bench top apparatus with pump (b).

Both components managed similar loads, but the pump did so with less input power, as expected. The cooling loop resulted in a COP of 44 with this configuration.

Another advantage of the pump was its compact size compared with the cumbersome fan housing. Limiting components on this bench-top setup included the size of the condenser and air flow across the condenser. Other broader issues that became apparent with this test were the flow passages through the motor housing and conduction-heat transfer from the motor windings to the refrigerant passages. These issues would need to be given consideration during motor-design stages when the R-134a loop-cooling method is being implemented.

For the second setup, a loop was attached to the high-pressure condenser section of a commercially available automotive AC system from a full-size sedan with a 9-kW capacity. The loop-inlet line was placed at the lowest elevation possible to ensure the pump inlet would remain flooded with liquid without undue increases in system inventory. A $1-\mathrm{kW}$ test load was initially used to prove loop operation. The loop maintained the test load near $30^{\circ} \mathrm{C}$. This specific system could also have handled a larger load, but these tests were not necessary for the proof-of-concept prototype.

After running, the loop was isolated from the stock AC system. The AC system produced adequate cooling after this test, which showed the loop had no adverse effect on AC system refrigerant-inventory behavior or refrigerant migration.

The final test involved operation of both systems simultaneously. The loop cooled the 1-kW test load and maintained it near $40^{\circ} \mathrm{C}$. This increase in temperature, as compared to the loop-only scenario, is a result of the increase in condenser-heat load when the compressor is running. 
When the loop runs by itself, the condenser pressure is around $650-720 \mathrm{kPa}$. When the AC system and loop are running simultaneously, the condenser pressure is $\sim 1 \mathrm{MPa}$. One key element in obtaining these stable results was to provide increased air flow across the condenser. For all tests, the condenser inlet air temperature was ambient laboratory temperature, $\sim 25^{\circ} \mathrm{C}$.

Overall, the floating-loop system met the initial design goals; however, these tests revealed several issues with the loop/AC dynamics. Oil traps and liquid refrigerant traps had been unintentionally created during the integration of the loop into the automotive AC system. Liquid migration and flash boiling during the cycling of the compressor was also discovered to be a problem. Dry-out of the load is considered to be a major issue when designing for direct cooling of power electronics. If the liquid level drops significantly in the load or dry-out occurs, loss of two-phase cooling occurs, and superheated vapor is produced, which results in significantly reduced heat transfer and rapidly increasing junction temperatures.

To correct these problems, four major revisions were implemented:

- Move the check valve downstream of the oil separator in a horizontal position.

- Move the solenoid valve closer to the condenser outlet.

- Move the evaporator fan to blow out of the case instead of through the condenser. Add dedicated condenser fans (Figure 2).

- Move the pump position within the loop to upstream of the filter and flow meter.

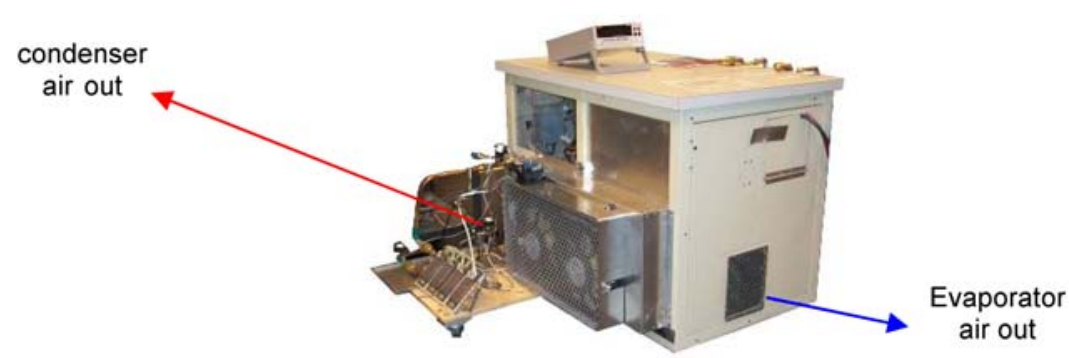

Figure 2. Revised floating loop attached to full-size sedan AC system in a cabinet.

During these changes, the original pump performance dropped due to unrelated pump motor electrical issues. One major design challenge had also been the electrical connections to the pump. A new pump was installed with commercially available high-pressure electrical terminals.

\section{Results}

After these changes were implemented, the loop shown in Figures 2 and 3 was retested at $1 \mathrm{~kW}$, $1.5 \mathrm{~kW}$, and $\sim 2 \mathrm{~kW}$. The $1-\mathrm{kW}$ load had similar results as previous tests. Repeated tests of the cooling loop by itself resulted in maintaining the load temperature around $35^{\circ} \mathrm{C}$ with $\sim 1.5 \mathrm{~kW}$ of heat.

The AC unit was incorporated with the loop-test load at $1 \mathrm{~kW}$ and at $1.5 \mathrm{~kW}$, and the load was maintained at 37 and $41^{\circ} \mathrm{C}$, respectively. This slightly higher temperature was expected because of the increase in operating pressure and, thus, higher saturation temperature. The flash-boiling effects were minimal during these tests, and no risk of load dry-out was obvious. The pump was at full power during these operations. When the $1.5-\mathrm{kW}$ load was decoupled from the AC system, the floating-cooling loop returned to $34^{\circ} \mathrm{C}$.

For test runs at up to $1960 \mathrm{~W}$, the floating-cooling loop maintained the load near $38^{\circ} \mathrm{C}$. When the $\mathrm{AC}$ was coupled to the loop, the system continued to run well with the load surface temperature at $49^{\circ} \mathrm{C}$ with full pump power. When the AC was turned off, the flash-boiling effects appeared to be minimal. 


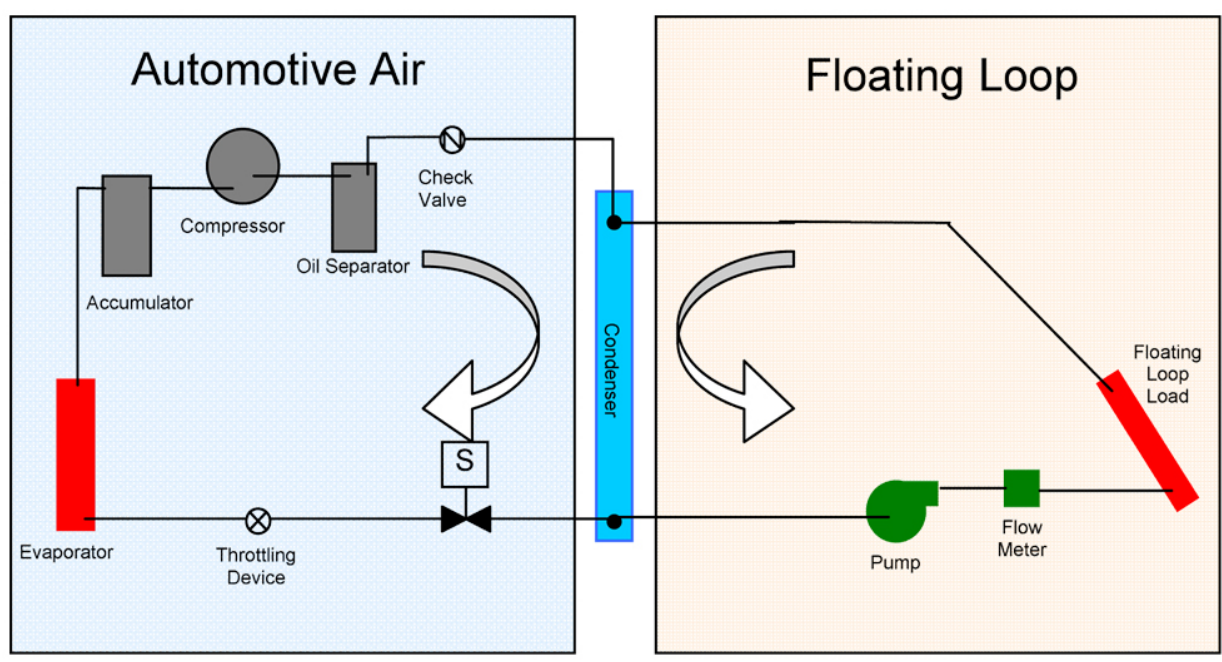

Figure 3. Floating loop/AC schematic with revisions.

\section{System dynamics}

To ensure a more robust design, the system instabilities previously mentioned were examined. When the pump was not at full power, several modes of system failure could arise: the pump would not supply enough liquid refrigerant to the loop in order to keep it wet, the differential pressure across the loop would collapse and cause backflow, or the cycling of the compressor would cause dry-out. During typical operation at $22^{\circ} \mathrm{C}$, four operating scenarios were encountered.

The four operating scenarios are steady operation of floating loop, steady operation with automotive $\mathrm{AC}$, transient start-up of automotive AC with loop, and the cycling off of the automotive air while maintaining loop operation. Each case presents unique operating requirements for which control methods are proposed and future design issues are discussed in the ORNL report.

\section{ORNL's Novel Silicon Die Mounting and New Capacitor Design}

The current status of the technology of conventional die mounting of the inverter is shown in Figure 4. This technology was chosen as the baseline for the development comparison. The heat flux path of the conventional die-mounted inverter is indicated by the arrow shown in Figure 5. There are multiple layers of thermal resistance in a conventional die-mounted inverter.

\section{Phase A \\ Phase C}

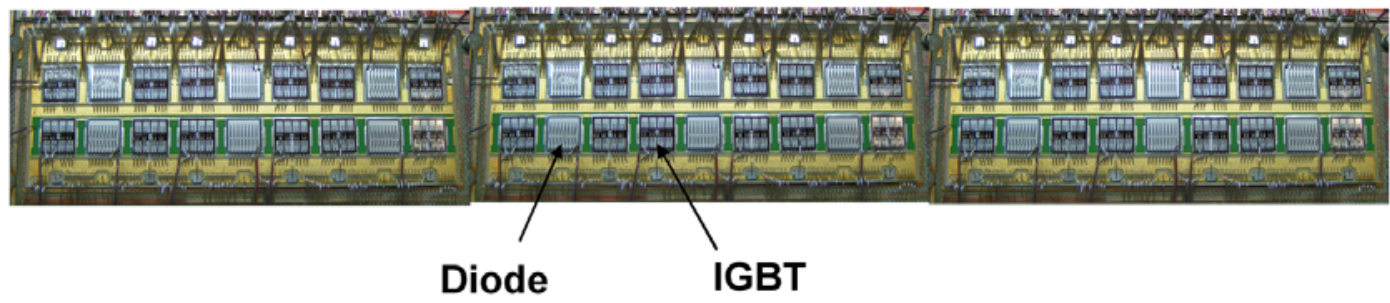

Figure 4. Conventional die mounting of inverter. 


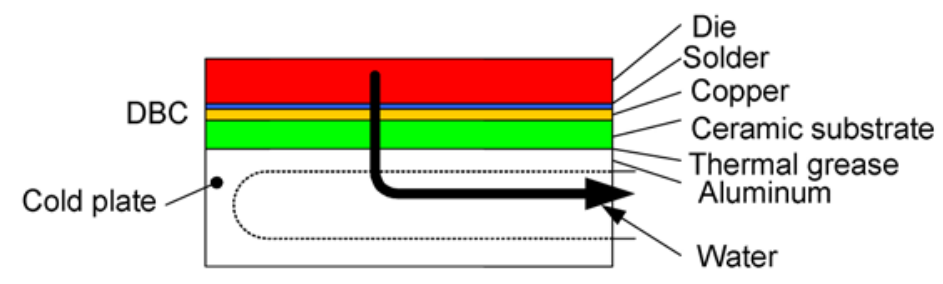

Figure 5. Arrow indicates the conventional heat flux path of the conventional die-mounting inverter.

The specific technical problem(s) addressed at ORNL for improving die cooling is to change the inverter packaging design for direct-contact, two-phase cooling and implement the design in the floating loop. The specific measurement at the end of the project is to reduce the inverter size to one-third of the 2005 Semikron inverter size with the same power rating.

The cascaded die mounting invented by ORNL is shown conceptually in Figure 6.

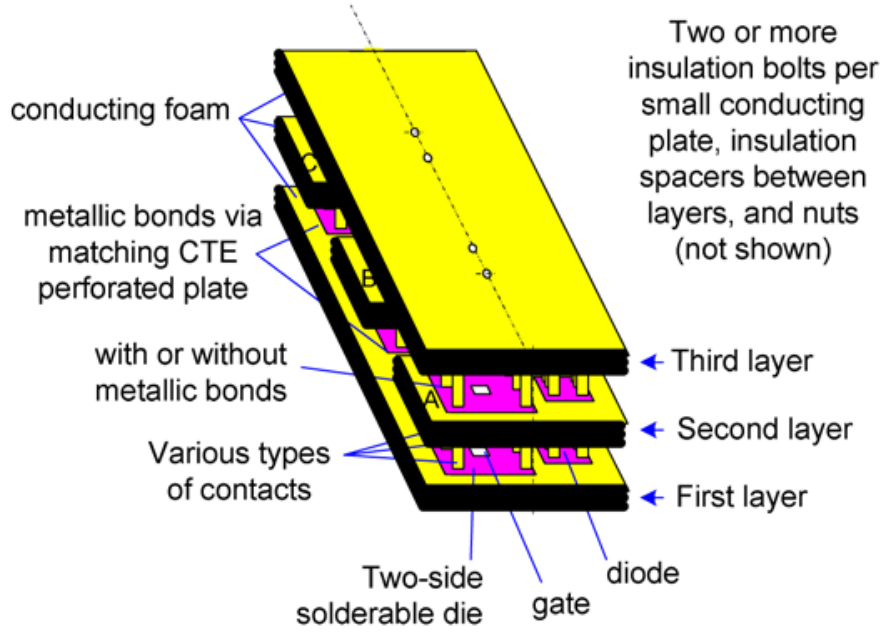

Figure 6. ORNL's cascaded die mounting concept.

Figure 7 shows copper foams used in the mounting. The reasons to use copper foam for the first prototype are (1) copper foam is a conductor without the need for heavy current wire bonds, (2) the dielectric cooling fluid can contact the die and the copper foam directly for two-phase heat transfer, and (3) it provides possibilities for thermal expansion control. This sample uses $25 \%$ density foam with 49 pores/in.

For the copper foam preliminary prototype, Figure 8 shows a two-side solderable silicon die (IXGD9265) obtained from IXYS Company. Its dimensions are $0.012 \times 0.284 \times 0.375$ in. A larger die obtained from Rockwell Scientific, shown in Figure 9, was also used for the copper foam die mounting.

Figure 10(a) shows the copper-foam cascaded die mounting inside a glass test tube filled with R134a refrigerant. Figure 10(b) shows that when the die is conducting electric current, two-phase cooling occurs. The heat flux of ORNL's preliminary copper-foam die mounting reaches $170 \mathrm{~W} / \mathrm{cm}^{2}$.

Figure 11 shows the ORNL 20\% density copper foam die cooling tests, and Figure 12 shows similar tests with $25 \%$ foam density. The heat flux reaches about $170 \mathrm{~W} / \mathrm{cm}^{2}$ in continuous operation. 

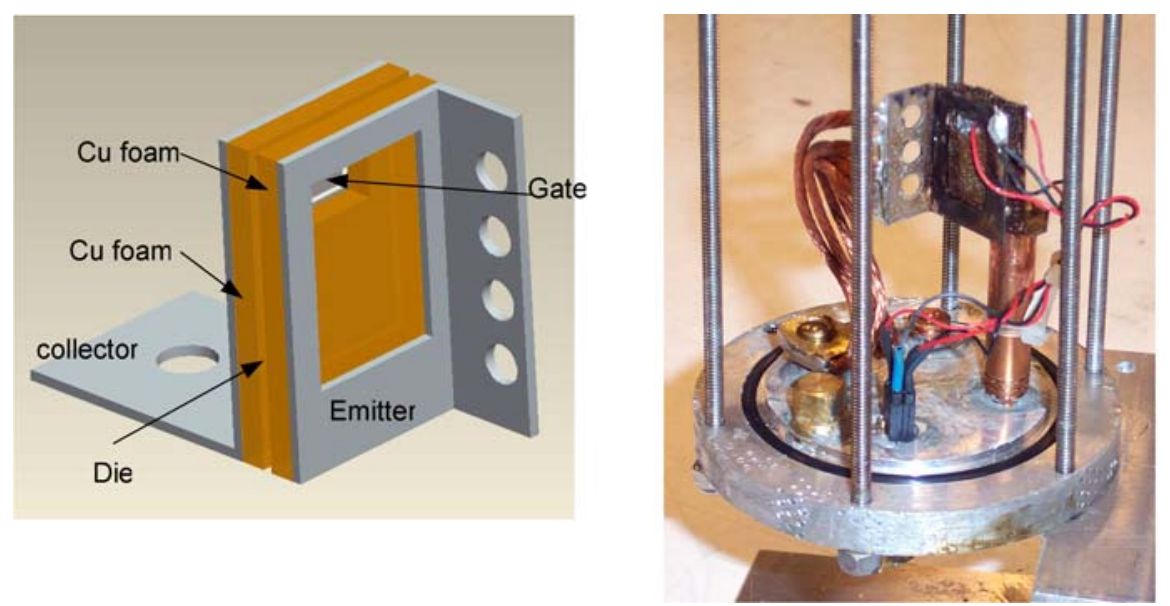

Figure 7. Copper foam is used as an example for cascaded die mounting.

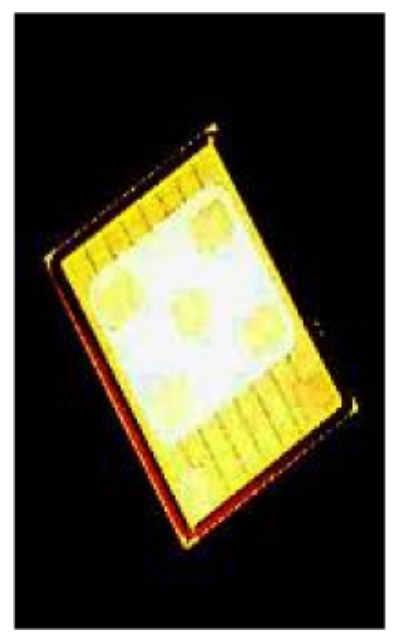

Figure 8. An IXYS two-side solderable die.

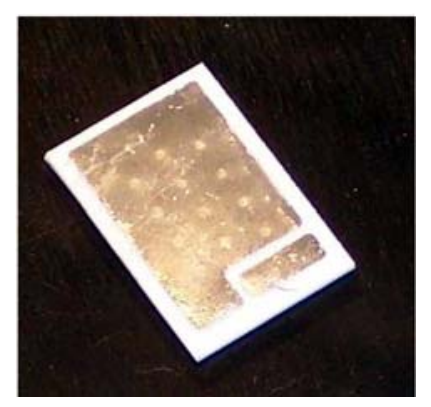

Figure 9. A two-side solderable die obtained from Rockwell Scientific. 
(a)

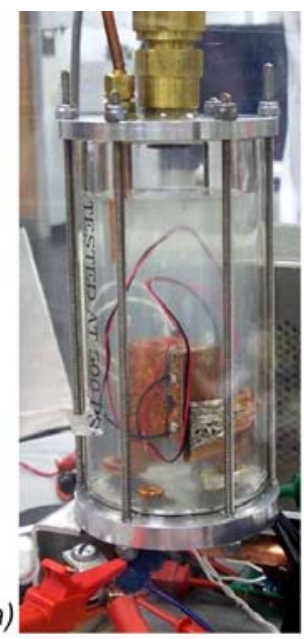

(b)

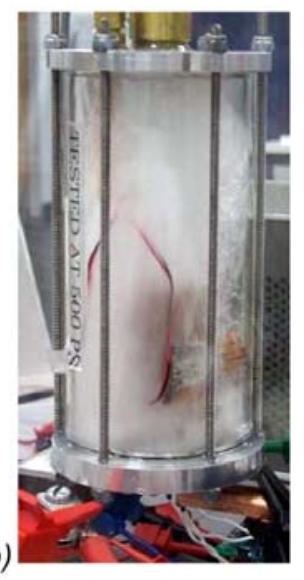

Figure 10. Prototype of copper-foam cascade die mounting (a). Two-phase cooling occurs when the die is loaded (b).

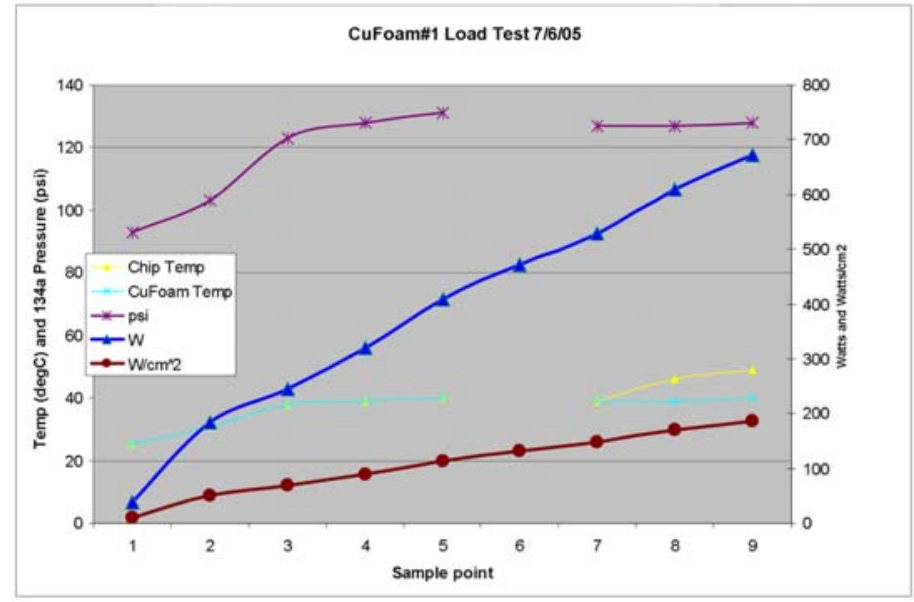

Figure 11. ORNL $20 \%$ density copper foam die cooling tests.

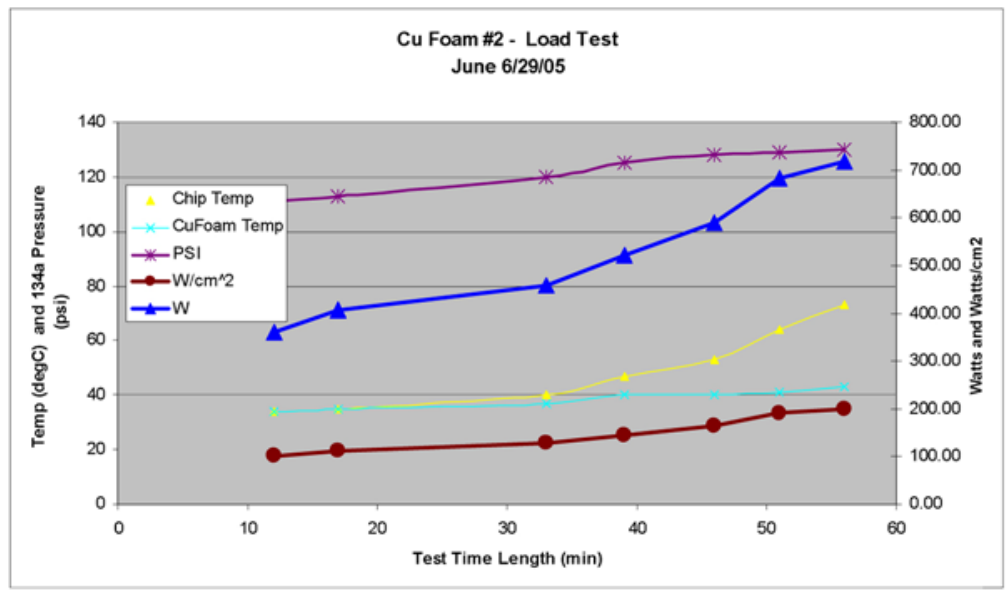

Figure $12.25 \%$ foam density test. 


\section{Film Capacitor Cooling}

It is known that the permissible ripple current of a film capacitor goes down when the ambient temperature of the capacitor goes up. Reducing the capacitor size cooling of the capacitor is important. ORNL has a patent pending on the direct cooling of film capacitors with refrigerants. The design of a new film capacitor prototype is shown in Figure 13.

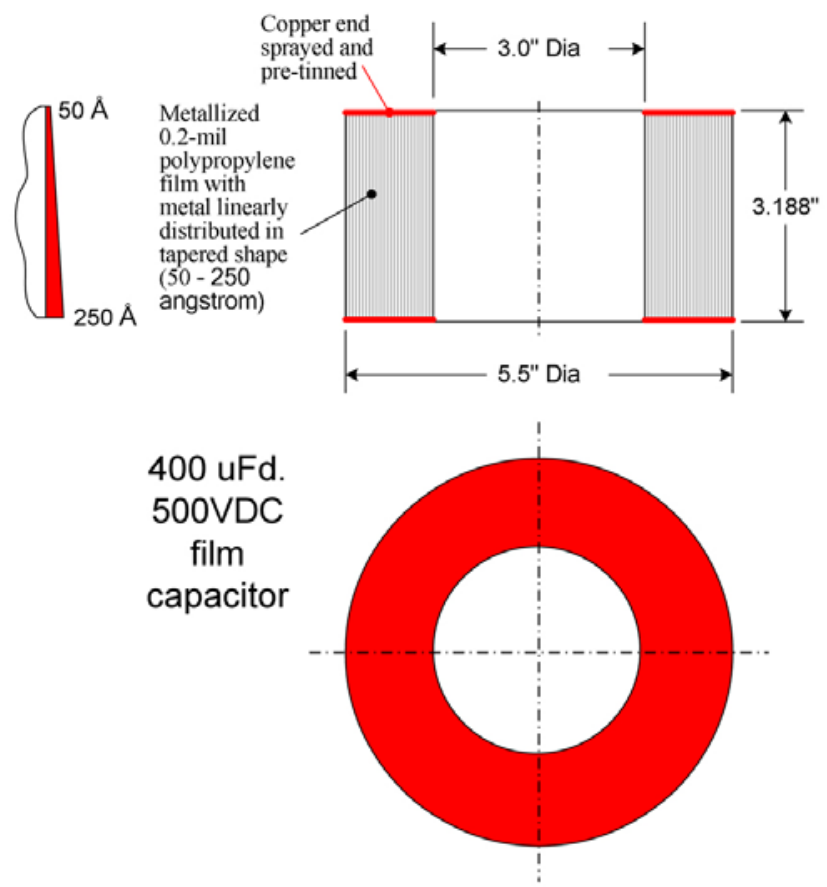

Figure 13. Example of an ORNL directly cooled film capacitor.

In this concept, the hollow center of the capacitor is the space available for the power electronic dies. The inverter assembly consisting of the capacitor, the dies, and other components are housed in a container allowing for direct refrigerant cooling. Figure 14 shows how the metal thickness on the film is linearly tapered with the thicker side positioned for external connections. Because the current is accumulating when it goes toward the connection side, this helps to make the current density more uniform in the metal.

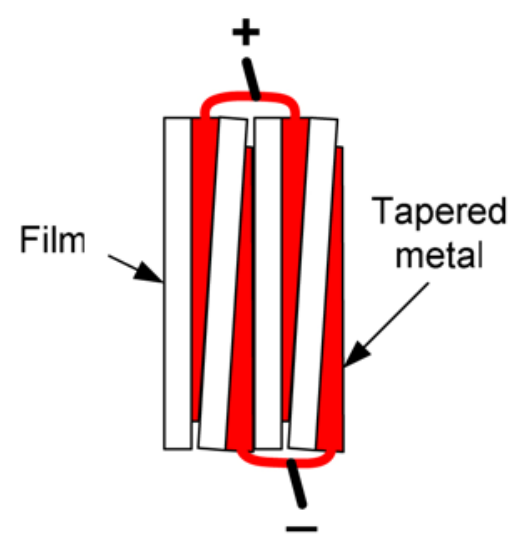

Figure 14. Tapered metal thickness on film. 


\section{Assessment of Emerging Two-Phase Cooling Technologies for Power Electronic Inverters}

Details of the assessment are presented in the Emerging Two-Phase Cooling Technologies for Power Electronic Inverters (Interim Report), ORNL/TM-2005/156. The report presents the findings of two single-loop, two-phase, water-ethylene-glycol (WEG) cooling systems and a double-loop, two-phase, transmission oil/dielectric fluid system through the tests of prototypes built by three subcontractors. The subcontractors are GT/Innovative Fluidics, RSC, and ISR. All three prototype cooling systems were implemented on a modified WEG coolant, single-phase, single-loop Semikron inverter. The $105^{\circ} \mathrm{C}$ WEG coolant was used in GT's VIBE system and in RSC's jet impingement cooling (JIC) system for their twophase, single-loop systems. ISR used $85^{\circ} \mathrm{C}$ transmission oil to cool a second-dielectric fluid loop for direct-die spray cooling. All three subcontractors' systems operate below 30-psi pressure.

In this investigation, the potentials and problems of different two-phase technologies proposed by the three subcontractors can be clearly seen. All test results presented in the report were witnessed by ORNL.

\section{ORNL's assessment of GT/Innovative Fluidics, single-loop, two-phase JIC system}

The prototype required roughly $760 \mathrm{VA}$ (720 VA plus fan motor) of apparent power to cool a 380-W loss during a dc test. Figure 15 shows severe erosion that appeared on the surfaces of the piezoelectric vibrators and on the metal-cooling chamber of the inverter after an hour of testing. Very little vaporization occurred due to the high-boiling temperature of the WEG and the low permissible junction temperature $\left(125^{\circ} \mathrm{C}\right)$ of the $\mathrm{Si}$ dies. The WEG boils at $106^{\circ} \mathrm{C}$ at atmosphere pressure. The boiling temperature increases as the pressure increases.

Although the VIBE technology failed to show its effectiveness from the tests, it may still be worth revisiting when the permissible junction temperature of the dies can be raised to allow more vaporization. However, to use this technology, the input power to the piezoelectric vibrators and the cooling fan would have to be reduced and the erosion problem solved.

\section{ORNL's assessment of RSC single-loop, two-phase JIC system}

RSC developed a closed-loop jet-impingement cooling (JIC) technique with $105^{\circ} \mathrm{C}$ WEG temperature. The targeted micro-jet array-impingement technique was implemented on a 450-V (dc link voltage) 400-A (rms current) Semikron module. Figure 16 shows the schematic of the closed-loop WEG micro-jet array impingement cooling system for the Semikron inverter.

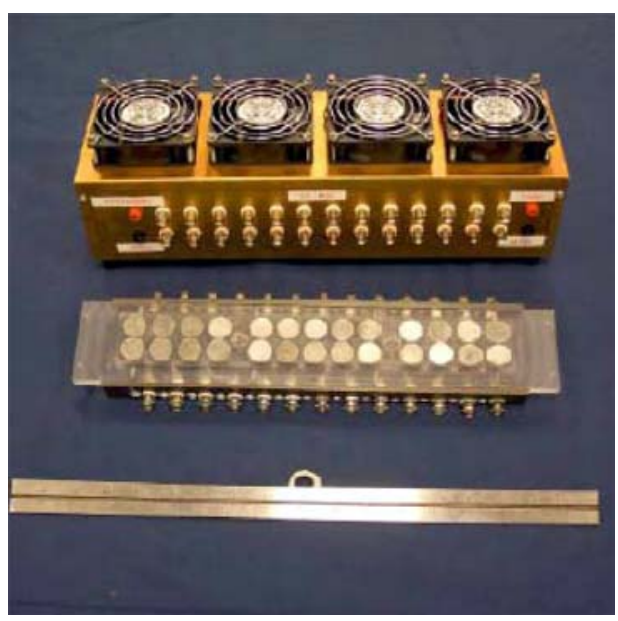

Figure 15. Photograph of Array B-2.4-MHz actuators. 


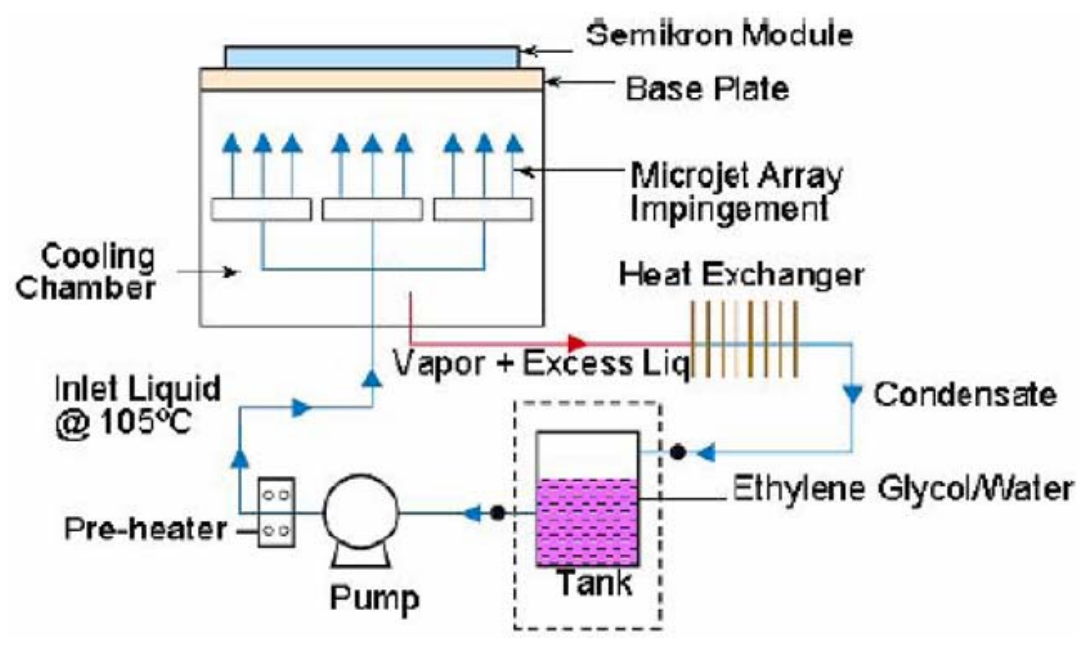

Figure 16. A schematic of the closed-loop WEG micro-jet array impingement cooling system for the Semikron inverter.

The dc testing of the module with $\sim 105^{\circ} \mathrm{C}$ coolant at 2.25 -gpm flow rate (corresponding pressure drop $24 \mathrm{psi}$ ) demonstrated $1623 \mathrm{~W}$ of heat dissipation at an allowable $20^{\circ} \mathrm{C}$ device temperature rise. This translates to a chip-level power dissipation density of $56 \mathrm{~W} / \mathrm{cm}^{2}$, an estimated $1.8 \times$ improvement over the state-of-the-art Semikron cold-plate thermal control scheme prorated to the same $105^{\circ} \mathrm{C}$ coolant temperature for comparison.

RSC also estimated that by cutting off part of the heat sink to expose the direct-bonded copper (DBC) underneath the die to the jets, the heat flux might potentially reach $90 \mathrm{~W} / \mathrm{cm}^{2}$.

\section{ORNL's assessment of ISR double-loop, two-phase spray cooling system}

Figure 17 shows the ISR double-loop, two-phase spray cooling system. It is designed to use an $85^{\circ} \mathrm{C}$ transmission oil loop to cool a second loop containing a dielectric liquid for spray cooling. The final ISR report and ORNL's assessment will be completed upon final testing of the ISR modules in early 2006

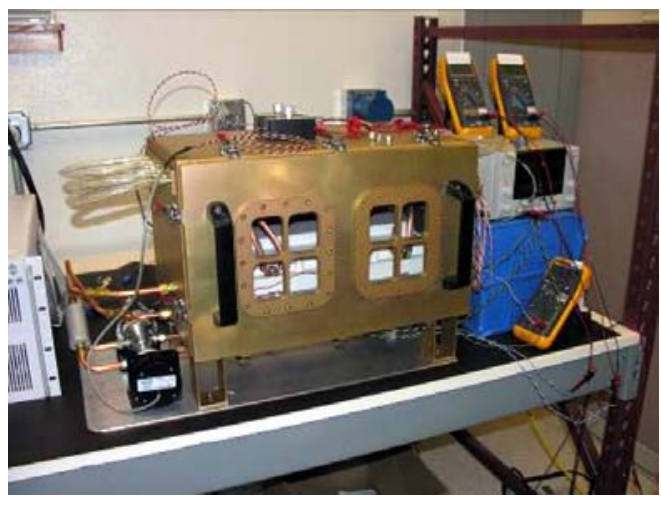

Figure 17. ISR spray cooling system for the Semikron inverter. 


\section{Refrigerant Properties}

Detailed basic refrigerant properties are included in the report Potential Refrigerants for Power Electronics Cooling, ORNL/TM-2005/219.

Cooling power electronic components in a refrigerant requires that the refrigerant meet certain constraints. First, the refrigerant must be environmentally friendly. Second, the refrigerant must be effective at two-phase cooling. This implies that the refrigerant has appropriate thermo-physical properties. Finally, the refrigerant must have high-dielectric strength. The major conclusions are outlined by the following tables.

A major issue with cooling power electronics directly with refrigerant is the dielectric strength. Highdielectric strength of a fluid enables it to resist current under high-voltage potential. Because power electronics are completely exposed in the refrigerant, the fluid must not permit the flow of current to short-circuit and damage power electronics and other components, or to cause malfunction of power electronics control circuits.

\section{Environmental impact}

Table 1 depicts data concerning environmental, flammability, and health concerns of the refrigerants under investigation. Chlorofluorocarbons (CFCs) have the worst environmental impact with a large ozone-depleting potential (ODP) and global-warming potential (GWP) (100 years), and has been eliminated from use through the Montreal Protocol. The only other refrigerants to have ODP are the hydrochlorofluorocarbons (HCFCs). Although HCFCs have a much lower ODP value, the Copenhagen Amendments to the Montreal Protocol call for ending production of ozone-depleting compounds by 2010 [1], eliminating the use of HCFCs in the future.

Figure 18 graphically represents the GWP of different refrigerants in reducing order. Clearly from Figure 18, CFCs and perfluorocarbons (PFCs) are undesirable refrigerants in terms of the environment with significantly larger GWP values compared with other refrigerants. A noteworthy observation is the low GWP values of hydrofluoroether (HFE), hydrocarbons (HC), and methylsiloxane (MS) refrigerants. Along with low values of GWP, these refrigerants have no ODP and low environmental impact. Still, water is the optimum refrigerant with no harmful effects to the environment.

\section{Two-phase cooling}

Figure 19 depicts the latent-heat values of the refrigerants in reducing order. These and other important physical properties of the refrigerants being examined are listed in Table 2. Based on observation of Figure 19, water has the highest two-phase heat-transfer potential, by more than a factor of 6 . For this reason, water is often used in indirect cooling because the water does not come in contact with the electronics. The next highest refrigerants with the greatest two-phase heat-transfer potential are the HC blends of Table 2. The lowest potential heat-transfer refrigerants are the HFE and PFC of Table 2 with latent-heat values smaller than $1 / 23$ that of water.

In terms of the normal-boiling point, the refrigerants most suited for cooling electronics are those with boiling-point temperatures in the range $20-80^{\circ} \mathrm{C}$. From Table 2, these refrigerants include PFCs and HFE type refrigerants. HCFC, hydrofluorocarbon (HFC), CFC, and HC refrigerants require a mediumpressure container to have both liquid and vapor phases due to the low values of the normal-boiling points. The MS refrigerants have high normal-boiling point temperatures requiring a slightly subatmospheric pressure on the container. This gives PFC and HFE refrigerants an advantage in terms of safety, operation, and cost. 
Table 1. Environmental, ignitability, and health data

\begin{tabular}{|c|c|c|c|c|c|c|c|c|c|}
\hline \multirow[t]{2}{*}{$\begin{array}{l}\text { Cooling Fluid } \\
\text { Description }\end{array}$} & \multirow[b]{2}{*}{ Company } & \multirow[b]{2}{*}{ Product Name } & \multirow{2}{*}{$\begin{array}{c}\text { Flammable? } \\
\text { Yes/No }\end{array}$} & \multirow{2}{*}{$\begin{array}{c}\begin{array}{c}\text { Auto } \\
\text { Ignition }\end{array} \\
\text { Point } \\
\left({ }^{\circ} \mathrm{C}\right)\end{array}$} & \multirow{2}{*}{$\begin{array}{c}\text { Lower Expl. } \\
\text { Limit }\end{array}$} & \multicolumn{2}{|c|}{ Enviornmental } & \multirow{2}{*}{ 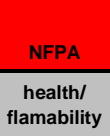 } & \multirow{2}{*}{$\begin{array}{c}\text { HMIS } \\
\text { health/ } \\
\text { flamability }\end{array}$} \\
\hline & & & & & & $\begin{array}{c}\text { ODP } \\
\text { (R11=1) }\end{array}$ & $\begin{array}{c}\text { GWP } \\
(100 \mathrm{yr})\end{array}$ & & \\
\hline $1,1,1,2$ tetra fluorethane & Various & HFC-134a & No & 750 & NA & 0 & 1300 & $1 / 0$ & $1 / 0$ \\
\hline $\begin{array}{l}\text { 1,1-dichloro-1- } \\
\text { fluoroethane }\end{array}$ & Various & HCFC-141b & Yes & 325 & 6 & 0.086 & 700 & $1 / 0$ & $1 / 0$ \\
\hline $\begin{array}{l}\text { 1-chloro-1,1- } \\
\text { difluoroethane }\end{array}$ & various & HCFC-142b & Yes & 632 & 9 & 0.043 & 2300 & $1 / 0$ & $1 / 0$ \\
\hline $\begin{array}{l}\text { Dichlorodifluoro } \\
\text { methane }\end{array}$ & Various & CFC-12 & No & $>750$ & NA & 0.82 & 10600 & $2 / 4$ & $2 / 4$ \\
\hline Chlorodifluoro methane & Various & HCFC-22 & No & NA & NA & 0.034 & 1700 & $2 / 1$ & $2 / 1$ \\
\hline perfluorocarbon & $3 \mathrm{M}$ & Fluoroinert FC-87 & No & NA & NA & 0 & 8900 & $3 / 0$ & $0 / 0$ \\
\hline perfluorocarbon & $3 \mathrm{M}$ & Fluoroinert FC-72 & No & NA & NA & 0 & 9000 & $3 / 0$ & $1 / 0$ \\
\hline perfluorocarbon & $3 \mathrm{M}$ & Fluoroinert FC-77 & No & NA & NA & 0 & 9000 & $3 / 0$ & $1 / 0$ \\
\hline hydrofluoroether & $3 \mathrm{M}$ & Novec HFE-7000 & No & 415 & NA & 0 & 400 & $3 / 1$ & $0 / 1$ \\
\hline hydrofluoroether & $3 \mathrm{M}$ & Novec HFE-7100 & No & 405 & NA & 0 & 320 & $3 / 1$ & $0 / 1$ \\
\hline hydrofluoroether & $3 \mathrm{M}$ & Novec HFE-7200 & No & 375 & 2.4 & 0 & 55 & $3 / 0$ & $1 / 0$ \\
\hline methylsiloxane & $\begin{array}{l}\text { Dow } \\
\text { Corning }\end{array}$ & Cleaning Agent 1 & Yes & 341.1 & 1.25 & 0 & $<10$ est & $1 / 3$ & \\
\hline $\begin{array}{c}\text { methylsiloxane } 60 \% \\
\text { w/alcohol } \\
\end{array}$ & $\begin{array}{c}\text { Dow } \\
\text { Corning } \\
\end{array}$ & Cleaning Agent 2 & Yes & 365 & 1.3 & 0 & $<10$ est & $1 / 3$ & \\
\hline De-lonized Water & & $\mathrm{H}_{2} \mathrm{O}$ & No & NA & NA & 0 & $<1$ est & & \\
\hline $\begin{array}{c}\text { (butane/isobutane/prop } \\
\text { ane) }\end{array}$ & Duracool & Duracool 12a & Yes & 891 & 1.95 & 0 & $\sim 20$ & $1 / 4$ & $1 / 4$ \\
\hline $\begin{array}{c}\text { hydrocarbon blend ( } \\
\text { propane/butane) }\end{array}$ & Enviro-safe & ES-12 & Yes & 863 & 1.9 & 0 & $\sim 20$ & $1 / 4$ & $1 / 4$ \\
\hline $\begin{array}{c}\text { fluorocarbon blend } \\
\text { (HFC-134a/HCFC-142b) }\end{array}$ & $\begin{array}{l}\text { Technical } \\
\text { Chemical } \\
\end{array}$ & Freeze 12 & No & $>150$ & $\begin{array}{c}\text { not } \\
\text { determined }\end{array}$ & 0.01 & 1500 & $2 / 2$ & $2 / 2$ \\
\hline
\end{tabular}

Global Warming Potential of Specified Refrigerants

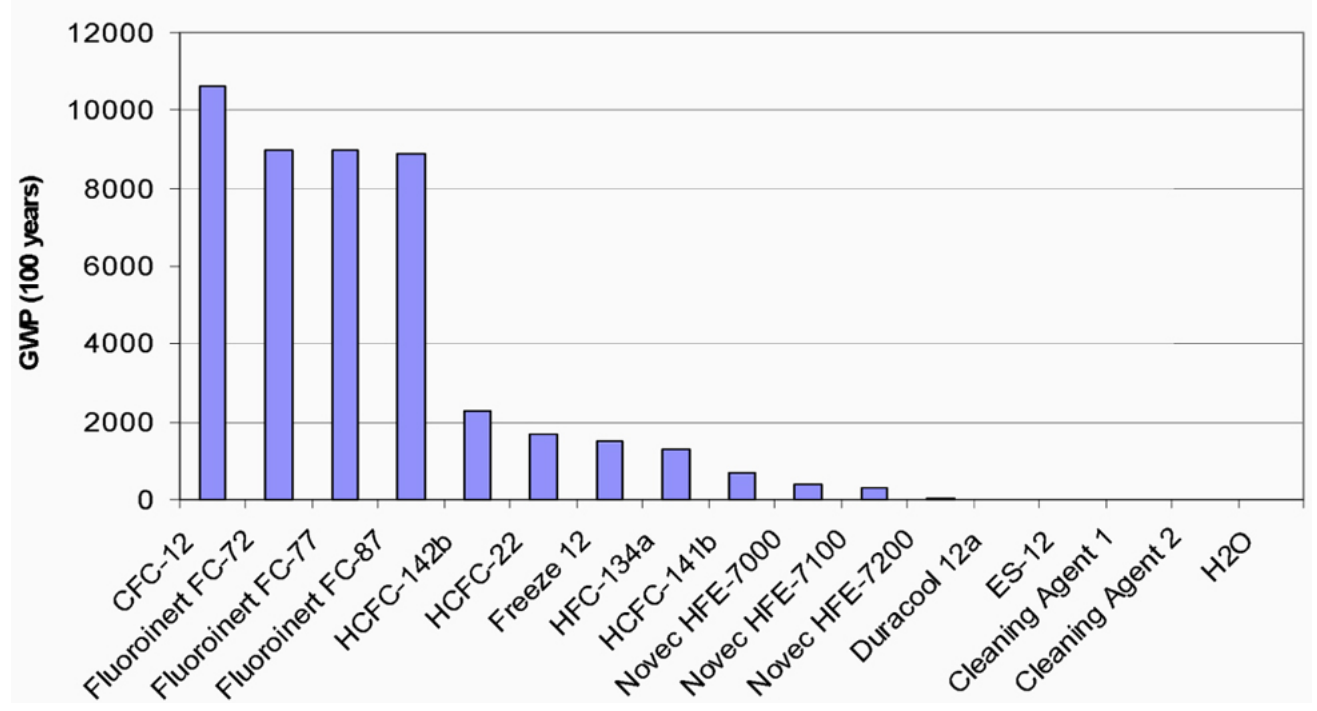

Figure 18. Refrigerant global warming potential. 
Latent Heat of Specified Refrigerants

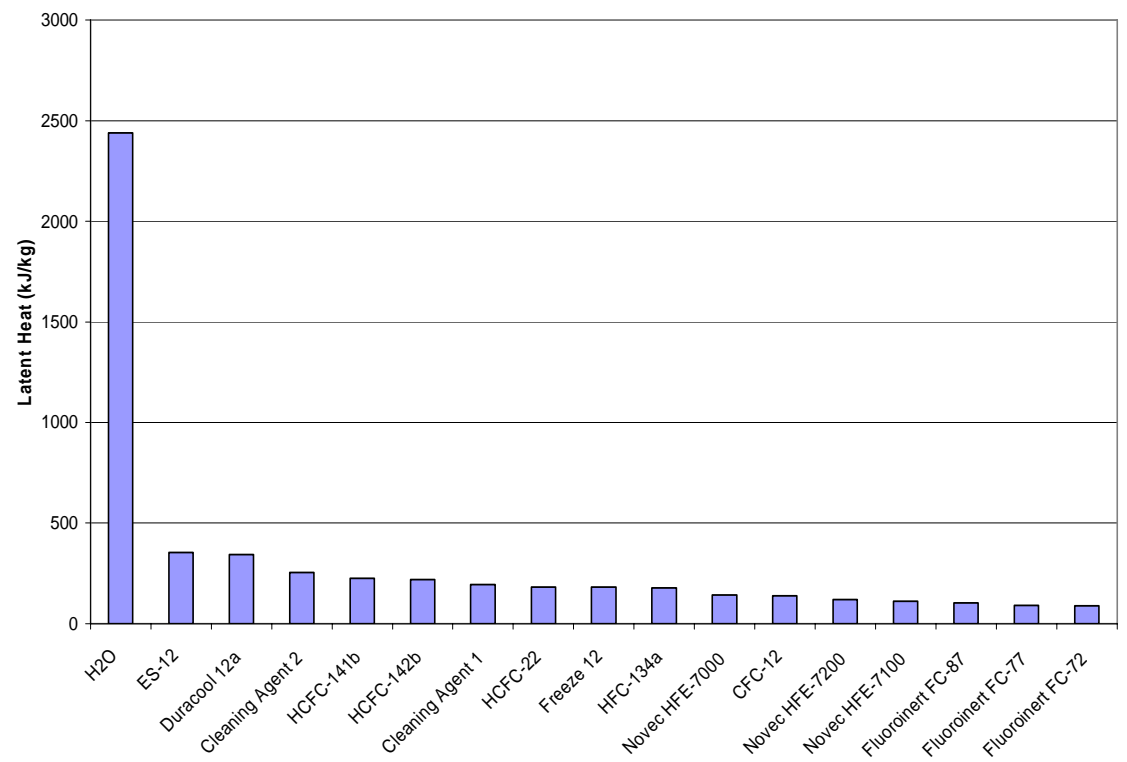

Figure 19. Latent heat at normal boiling point.

Table 2. Physical properties

\begin{tabular}{|c|c|c|c|c|c|c|}
\hline Cooling fluid description & & & $\begin{array}{c}\text { Latent } \\
\text { heat @ } \\
25^{\circ} \mathrm{C}\end{array}$ & $\begin{array}{c}\text { Specific } \\
\text { heat @ } 25^{\circ} \mathrm{C}\end{array}$ & $\frac{\text { Density @ }}{\underline{25^{\circ} \mathrm{C}}}$ & $\begin{array}{c}\text { Normal } \\
\text { boiling } \\
\text { point }\end{array}$ \\
\hline & Company & Product name & $\mathbf{k J} / \mathbf{k g}$ & $\begin{array}{c}\text { Liquid } \\
(\mathrm{kJ} / \mathbf{k g} / \mathrm{K})\end{array}$ & $\begin{array}{l}\text { liquid } \\
\mathbf{k g} / \mathbf{m}^{3}\end{array}$ & ${ }^{\circ} \mathrm{C}$ \\
\hline $1,1,1,2$ tetra fluorethane & Various & HFC-134a & 178 & 1.4 & 1210 & -26 \\
\hline 1,1-dichloro-1-fluoroethane & Various & HCFC-141b & 226 & 1.15 & 1234 & 32 \\
\hline 1-chloro-1,1-difluoroethane & various & HCFC-142b & 217.8 & 1.3 & 1.12 & -10 \\
\hline Dichlorodifluoro methane & Various & CFC-12 & 139 & 1 & 1311 & -29.8 \\
\hline Chlorodifluoro methane & Various & HCFC-22 & 182.7 & 1.26 & 1191 & -40.8 \\
\hline perfluorocarbon & $3 \mathrm{M}$ & Fluoroinert FC-87 & 103 & 1.1 & 1650 & 30 \\
\hline perfluorocarbon & $3 \mathrm{M}$ & Fluoroinert FC-72 & 88 & 1.1 & 1700 & 56 \\
\hline perfluorocarbon & $3 \mathrm{M}$ & Fluoroinert FC-77 & 89 & 1.1 & 1780 & 97 \\
\hline hydrofluoroether & $3 \mathrm{M}$ & Novec HFE-7000 & 142 & 1.3 & 1400 & 34 \\
\hline hydrofluoroether & $3 \mathrm{M}$ & Novec HFE-7100 & 112 & 1.17 & 1520 & 61 \\
\hline hydrofluoroether & $3 \mathrm{M}$ & Novec HFE-7200 & 119 & 1.22 & 1420 & 76 \\
\hline methylsiloxane & Dow Corning & Cleaing Agent 1 & 194 & 1.72 & 850 & 100 \\
\hline methylsiloxane $60 \%$ w/alcohol & Dow Corning & Cleaning Agent 2 & 255 & & 770 & 98 \\
\hline De-Ionized Water & & $\mathrm{H}_{2} \mathrm{O}$ & 2440 & 4.13 & 1002 & 100 \\
\hline $\begin{array}{c}\text { hydrocarbon blend } \\
\text { (butane/isobutane/propane) }\end{array}$ & Duracool & Duracool 12a & 343.5 & 2.56 & 526 & -31.5 \\
\hline $\begin{array}{l}\text { hydrocarbon blend } \\
\text { (propane/butane) }\end{array}$ & Enviro-safe & ES-12 & 354.2 & 2.574 & 530 & -30.4 \\
\hline $\begin{array}{c}\text { fluorocarbon blend } \\
\text { (HFC-134a/HCFC-142b) }\end{array}$ & $\begin{array}{l}\text { Technical } \\
\text { Chemical } \\
\end{array}$ & Freeze 12 & 180.7 & 1.4 & 1189 & -25 \\
\hline
\end{tabular}




\section{Dielectric Strength}

Because power electronics are to be cooled using two phases, both the liquid and vapor phases require dielectric-strength testing. The testing temperatures are based on the expected operating temperature of the two-phase mixtures. Saturated liquid and vapor denote the test pressure of the mixtures. To replicate the actual placement of power electronics in the mixtures, sharp electrodes are used in the dielectricstrength test. The dielectric strengths of the refrigerants are given in Table 3.

Table 3. Dielectric strength table

\begin{tabular}{|c|c|c|c|}
\hline & & Dielectric Strength & Dielectric Strength \\
\hline & & Liquid (kV/mil) & Vapor (kV/mil) \\
\hline Products & Product Name & $50^{\circ} \mathrm{C} /$ Saturated Liquid & $50^{\circ} \mathrm{C} /$ Saturated Vapor \\
\hline $1,1,1,2$ tetra fluorethane & HFC-134a & 7.2 & 6.7 \\
\hline & & $50^{\circ} \mathrm{C} /$ Saturated Liquid & $50^{\circ} \mathrm{C} /$ Saturated Vapor \\
\hline $\begin{array}{l}\text { 1-chloro-1,1- } \\
\text { difluoroethane }\end{array}$ & HCFC-142b & 5.9 & 5.4 \\
\hline & & $50^{\circ} \mathrm{C} /$ Saturated Liquid & $50^{\circ} \mathrm{C} /$ Saturated Vapor \\
\hline $\begin{array}{l}1,1 \text { - dichloro -1- } \\
\text { fluoroethane }\end{array}$ & HCFC-141b & 6.1 & 4.4 \\
\hline & & $50^{\circ} \mathrm{C} /$ Saturated Liquid & $50^{\circ} \mathrm{C} /$ Saturated Vapor \\
\hline hydrofluoroether & Novec HFE-7000 & 8.0 & 6.0 \\
\hline & & $80^{\circ} \mathrm{C} /$ Saturated Liquid & \\
\hline hydrofluoroether & Novec HFE-7100 & 8.1 & 7.1 \\
\hline & & $80^{\circ} \mathrm{C} /$ Saturated Liquid & $80^{\circ} \mathrm{C} /$ Saturated Vapor \\
\hline hydrofluoroether & Novec HFE-7200 & 7.7 & 3.8 \\
\hline & & $100^{\circ} \mathrm{C} /$ Saturated Liquid & $100{ }^{\circ} \mathrm{C} /$ Saturated Vapor \\
\hline methylsiloxane & Cleaning Agent 1 & 7.6 & 5.4 \\
\hline & & $100^{\circ} \mathrm{C} /$ Saturated Liquid & $100^{\circ} \mathrm{C} /$ Saturated Vapor \\
\hline $\begin{array}{l}\text { methylsiloxane } 60 \% \\
\text { w/alcohol }\end{array}$ & Cleaning Agent 2 & 6.3 & 4.4 \\
\hline
\end{tabular}

\section{Conclusions}

The concept of the floating refrigerant loop based on R-134a refrigerant cooling of high-heat-flux electronics was proved through experimental tests. For operation as part of a 9-kW automobile cooling system, the ORNL floating-loop system has been proven to function well at $2 \mathrm{~kW}$, with a very attractive COP of $>40$. The system capacity is easily scalable for larger loads. The operation of the floating cooling loop results in four major running scenarios. Each one has the potential to run stably and reliably, subject to appropriate component sizing and controls. A solenoid valve located close to the loop supply/condenser outlet to isolate the passenger AC components is crucial to the stable operation of the floating-cooling loop during AC compressor shutoff. Methods of increasing liquid inventory in the condenser and floating loop are required to maintain a wet load during compressor transients.

The ORNL cascaded die mounting that uses copper foam can achieve $170-\mathrm{W} / \mathrm{cm}^{2}$ heat flux value.

The work on direct cooling of film capacitors began, and a design for incorporation into the reduced size inverter was developed. 
The assessment of emerging two-phase cooling technologies for power electronic inverters gave objective conclusions through test data. Comparing with pure water, it is relatively harder to cool an inverter with WEG. The $105^{\circ} \mathrm{C}$ WEG was used for the GT's VIBE system as well RSC's JIC system for their two-phase, single-loop systems. The $85^{\circ} \mathrm{C}$ transmission oil was used by ISR to cool a seconddielectric fluid loop for direct-die spray cooling. All three subcontractors' systems operate below 30-psi pressure. The VIBE prototype took more energy to cool than its ability to extract. The JIC prototype reached $55 \mathrm{~W} / \mathrm{cm}^{2}$ heat flux. The ISR spray system will be tested in 2006.

The refrigerant property study indicates that the HFC (R134-a) remains a good candidate for hybrid power electronics cooling with good mechanical properties, strong dielectric strength, nonflammability, and wide acceptance in the auto industry. The HFE (Novec) fluids show good general properties for power electronics cooling, but are not presently used in automotive applications. The MS materials show excellent mechanical properties, but exhibit high flammability in conjunction with a high normal-boiling point at low system pressure and moderate dielectric strength. This creates the need for care in designing a safe oxygen-free system. The HCFC materials show moderate to poor dielectric strengths and are slated to be removed from production in the near future due to significant ODP and GWP.

\section{Future Direction}

The engineering improvements on the floating loop technology will be conducted for dynamic conditions as issues arise. The Venturi effect will be introduced into the cascaded die mounting for further increase of the heat flux. The specially made film capacitor will be tested with the reduced-size inverter. The ISR spray cooling prototype will be tested.

\section{Publications}

Floating Refrigerant Loop Based on R-134a Refrigerant Cooling of High Heat Flux Electronics, ORNL/TM-2005/223.

Report On Toyota Prius Motor Thermal Management, ORNL/TM-2005/33.

\section{Patents}

The available ORNL patents on thermal control are as follows:

- "Cascaded Die Mountings with Spring-Loaded Contact-bond Options," U.S. Patent 6,930,385, August 16, 2005.

- "Total Thermal Management System for Hybrid and Full Electric Vehicles," U.S. Patent 6,772,603, August 10, 2004.

- "Refrigerant Directly Cooled Capacitors," Patent pending No. 11/166,502.

- "Floating Loop System for Cooling Integrated Motors and Inverters," Patent pending No. 10/926,205.

- "Motor Frame and Cooling with Refrigerant and Liquid," Patent pending No. 60/565,461.

\section{$\underline{\text { Reference }}$}

1. The Challenge: Moving Away From HCFC Refrigerants, HRAI, The Heating, Refrigerating, and Air Conditioning Institute of Canada, 2005 (http://www.hrai.ca/hcfcphaseout/). 


\title{
2.2 Component Characterization
}

\author{
Principal Investigator: Larry Seiber \\ Oak Ridge National Laboratory \\ National Transportation Research Center \\ 2360 Cherahala Boulevard \\ Knoxville, TN 37932 \\ Voice: 865-946-1334; Fax:865-946-1400; E-mail: seiberle@ornl.gov \\ DOE Technology Development Manager: Susan A. Rogers \\ Voice: 202-586-8997; Fax: 202-586-1600; E-mail: Susan.Rogers@ee.doe.gov \\ ORNL Program Manager: Mitch Olszewski \\ Voice: 865-946-1350; Fax: 865-946-1262; E-mail: olszewskim@ornl.gov
}

\section{Objective}

As capacitor and magnet technologies mature, it is important to ascertain their limitations by subjecting the components to standardized tests to evaluate their capabilities. This testing will determine their reliability, degradation tendencies, and level of robustness when subjected to accelerated life testing conditions. Test results will help to predict whether the components are capable of meeting the 15-year service life requirement and help determine their ability to provide improvements in power electronics and motor designs to meet FreedomCAR goals.

\section{Approach}

In order to record and plot capacitor performance data, it has been necessary to develop a test stand that logs data at predetermined temperature extremes and controls thermal cycling between these extremes. This test stand includes an automated data acquisition system, an environmental chamber, and the necessary measuring instrumentation.

To understand how the new magnet technologies respond to thermal cycling, a hysteresis graph and $\mathrm{BH}$ curves of new magnet materials over the full range of temperatures from $-40^{\circ} \mathrm{C}$ to $140^{\circ} \mathrm{C}$ will be plotted.

\section{Major Accomplishments}

To obtain temperature cycling data, the thermal cycling process must continue uninterrupted for extended periods of time. To accomplish this long-term testing, an automated data acquisition system (DAC) using LABView was developed to control the environmental chamber and record data from the measuring instruments. The DAC will allow the operator to input upper and lower temperature limits as well as temperature increments to be used during the testing. The program will command a temperature to the environmental chamber; when that temperature has been reached, it will then monitor a capacitor parameter for stability. After this parameter is stable, the program will record the capacitor data from the measuring instruments at each of seven frequencies before another temperature step is commanded.

\section{$\underline{\text { Technical Discussion }}$}

\section{Capacitor Evaluation}

In FY 2005, the capacitor testing was limited to thermal cycling. Future testing will be done by applying a ripple current and bias voltage to the capacitors in the environmental chamber. The parameters recorded during the thermal cycling tests were capacitance value, dissipation factor, and equivalent series 
resistance (ESR). The temperature extremes for all tests were -40 and $140^{\circ} \mathrm{C}$. The typical increment for a temperature step was $20^{\circ} \mathrm{C}$. At each temperature increment, the parameters were measured at seven different frequencies: 1, 5, 10, 15, 20, 25, and $30 \mathrm{kHz}$. All capacitors were tested in an ESPEC environmental chamber, and their parameters were measured by an Agilent 4284A LCR meter.

\section{Data acquisition system}

The data acquisition system consists of a Dell Precision 380 Workstation running LABView 7.1. At the beginning of the thermal cycling test, the program turns on the environmental chamber, commands a temperature, and begins monitoring the chamber temperature to determine when that temperature has been reached. After the target temperature has been reached and a pre-programmed delay time has been met, the program sends a command to the LCR meter to output the first test frequency and monitors the ESR from the capacitor under test. When that value is stable, the data point is recorded and the next parameter is monitored and recorded.

After all parameters-ESR, dissipation factor, and capacitance value-are recorded at the first test frequency, the command is given to the LCR meter to output the next test frequency; and the monitoring and recording process is repeated. After data are recorded at all seven frequencies, the next temperature step is commanded to the environmental chamber, the frequencies are scanned again, and the parameters are recorded. The number of temperature steps that can be entered into the LABView program is, for all practical purposes, unlimited. The only test limitation is imposed by the LCR meter, which allows only certain test frequencies to be used.

The pre-programmed delay discussed earlier is to ensure that the temperature of the dielectric has equalized once the target temperature of the environmental chamber has been reached. When the difference between the ambient temperature and the target temperature of the chamber is $40^{\circ}$ or greater, the delay is invoked when the target temperature is reached. This delay value is entered before the program starts monitoring the temperature. The value is determined by the operator and is based on the size of the capacitor. A larger capacitor requires a larger delay to ensure that the temperature of the dielectric is uniform. After this delay time has expired, the program monitors the ESR for a stable reading before the value is recorded to ensure the ESR readings are accurate.

Five types of capacitors were tested and will be reported on here. Pennsylvania State University supplied a multilayer glass ceramic capacitor. Sandi National Laboratories supplied a commercial polyester film and a commercial Teflon film, both manufactured by ASC. The commercial capacitors were tested, and Sandia will use the data to aid in the development of its new technologies. The fourth capacitor tested was a metallized proprietary experimental film capacitor supplied by Dearborn Electronics. The fifth was a module consisting of 16 capacitors in parallel. This capacitor module was manufactured by Electronics Concepts, Inc., and supplied by Semikron. It is currently in use in the Semikron 600-V SKAI inverter.

\section{Multilayer glass ceramic}

The multilayer glass ceramic capacitor is a $1-\mu \mathrm{F}, 500-\mathrm{Vdc}$ capacitor. It was tested from -40 to $140^{\circ} \mathrm{C}$ at $20^{\circ}$ increments. Figures 1 and 2 show the data at the temperature extremes. The capacitance value and ESR decrease as frequency increases at the temperature extremes, but the dissipation factor increases with frequency at the temperature extremes. The dissipation factor and ESR values are much lower at the higher temperature, but the capacitance value is higher at the higher temperature. 


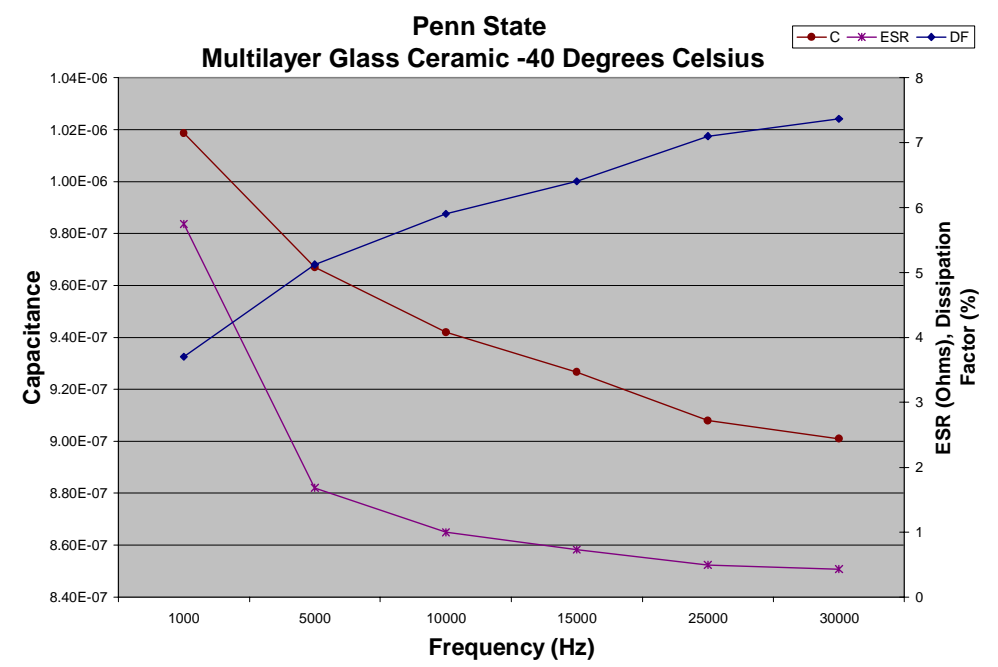

Figure 1. Multilayer glass ceramic at $-40^{\circ} \mathrm{C}$.

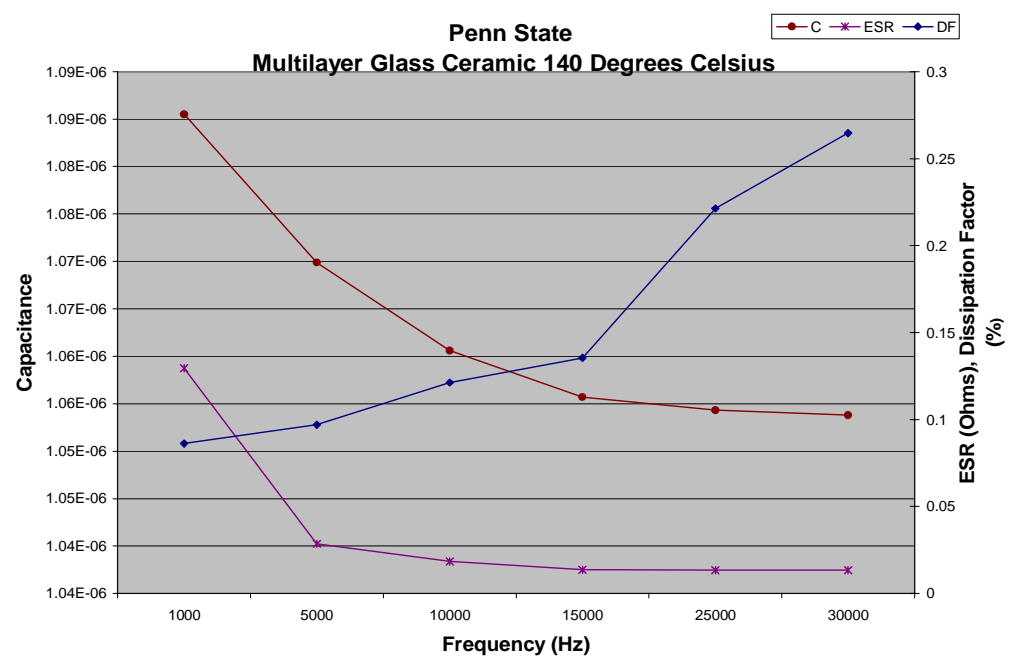

Figure 2. Multilayer glass ceramic at $140^{\circ} \mathrm{C}$.

\section{Commercial polyester film}

The polyester film capacitor is a $2-\mu \mathrm{F}, 600-\mathrm{Vdc}$ capacitor manufactured by ASC. It was tested from $-40^{\circ} \mathrm{C}$ to $140^{\circ} \mathrm{C}$ at $20^{\circ}$ increments between the extremes. Figures 3 and 4 show the data at the temperature limits. The dissipation factor and capacitance value increase with frequency at the temperature limits, but the ESR decreases as the frequency increases at both temperature limits. 


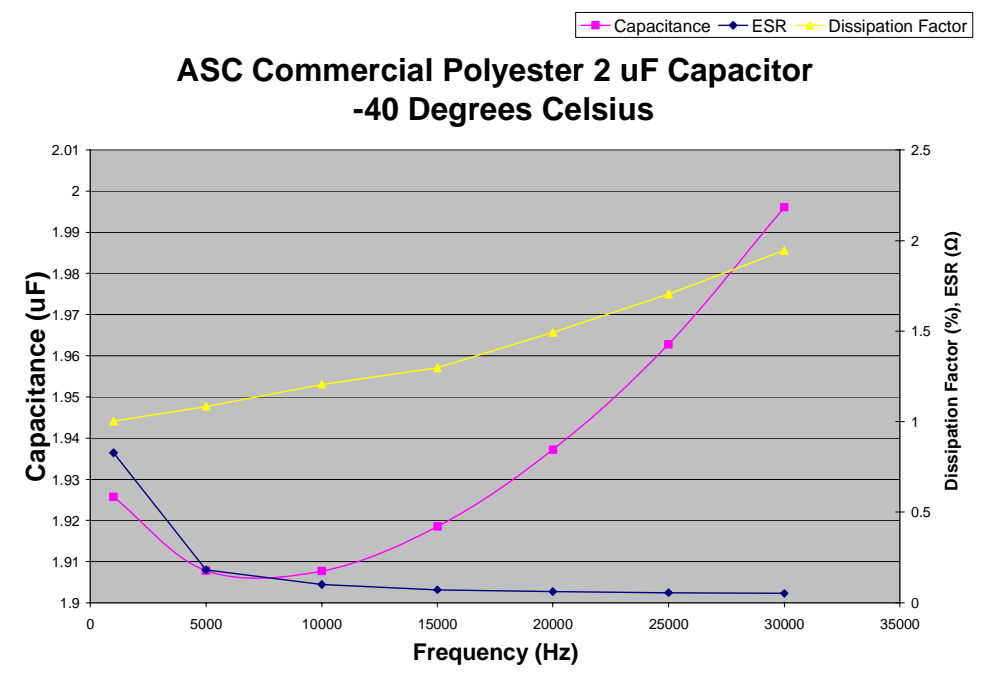

Figure 3. ASC commercial polyester capacitor at $-40^{\circ} \mathrm{C}$.

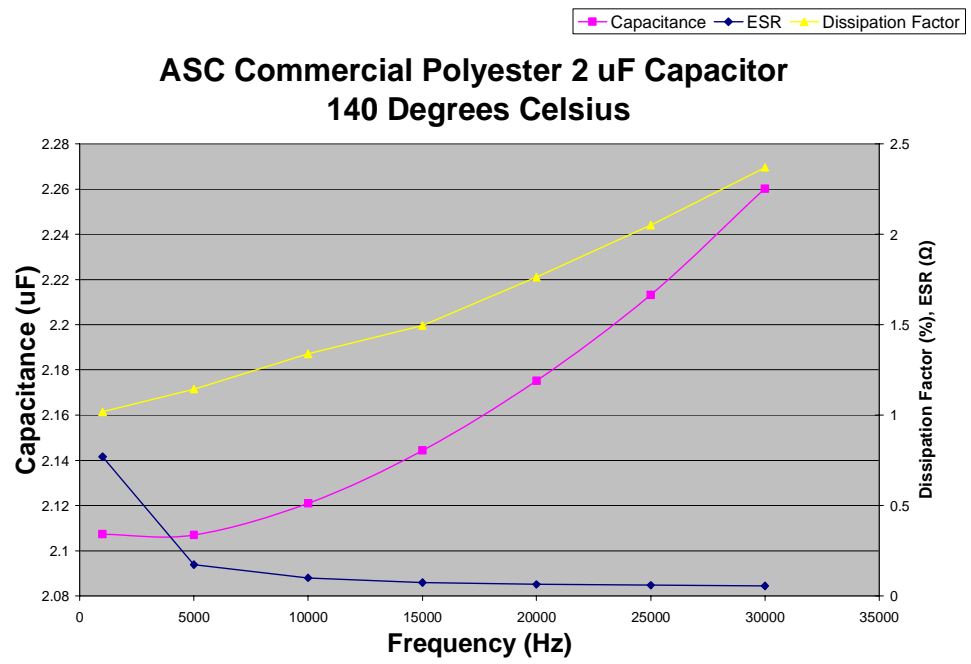

Figure 4. ASC commercial polyester capacitor at $140^{\circ} \mathrm{C}$.

\section{Commercial Teflon film}

The Teflon film capacitor tested is a $0.1-\mu \mathrm{F}, 600-\mathrm{Vdc}$ capacitor, also manufactured by ASC. It was also tested from -40 to $140^{\circ} \mathrm{C}$ at $20^{\circ}$ increments. The ESR and dissipation factor are very low on this capacitor at both temperature limits. The ESR decreases as frequency increases, but the capacitance value and dissipation factor both increase with frequency (Figures 5 and 6). 


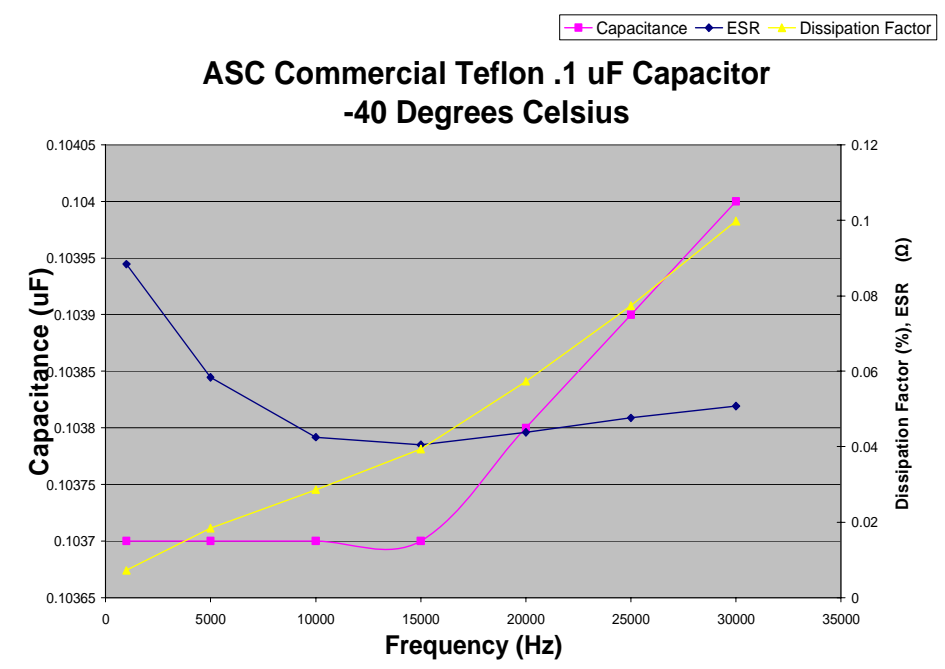

Figure 5. Commercial Teflon film capacitor at $-40^{\circ} \mathrm{C}$.

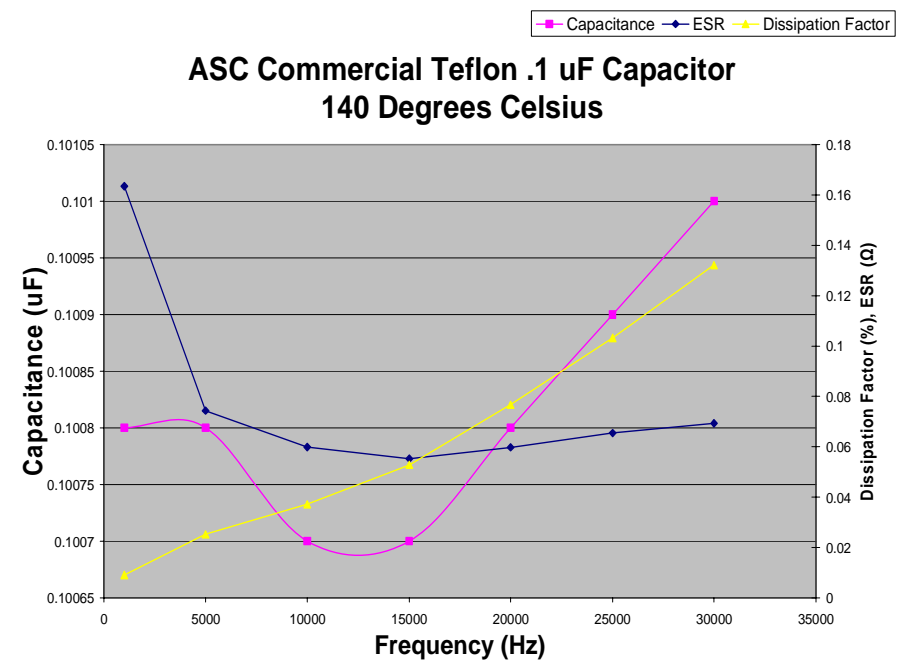

Figure 6. Commercial Teflon film capacitor at $140^{\circ} \mathrm{C}$.

\section{Dearborn $200^{\circ} \mathrm{C}$ capacitor}

The Dearborn high-temperature capacitor is a metallized proprietary experimental film $13-\mu \mathrm{F}$, $250-\mathrm{Vdc}$ capacitor. It was tested from -40 to $140^{\circ} \mathrm{C}$ at $20^{\circ}$ increments. The ESR on this capacitor is low at both temperature limits and decreases as frequency increases. Both the dissipation factor and capacitance value increase with frequency at both temperature limits. 


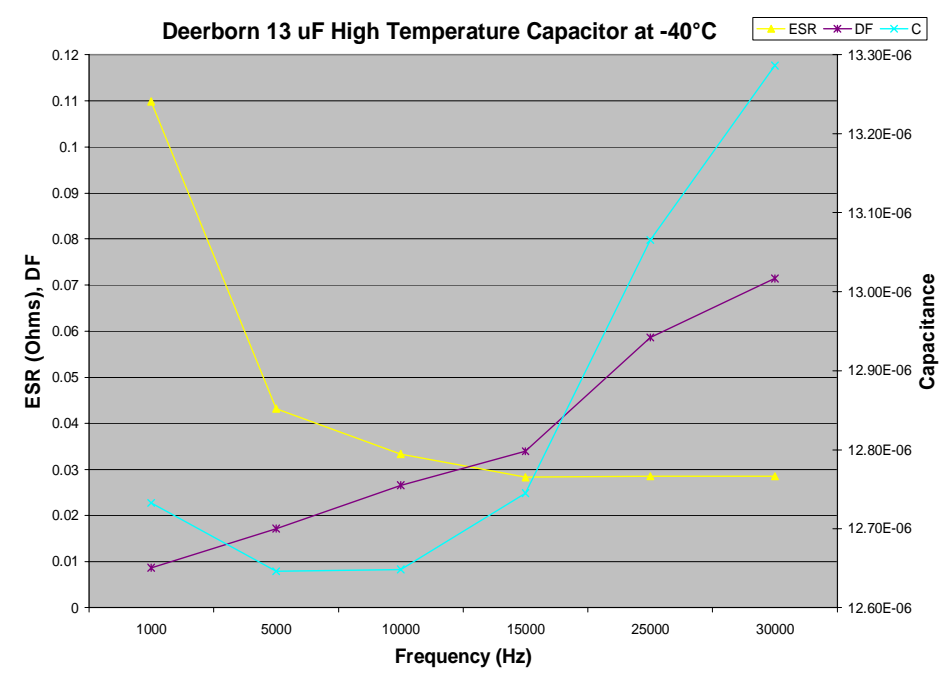

Figure 7. Dearborn metallized proprietary film capacitor at $-40^{\circ} \mathrm{C}$.

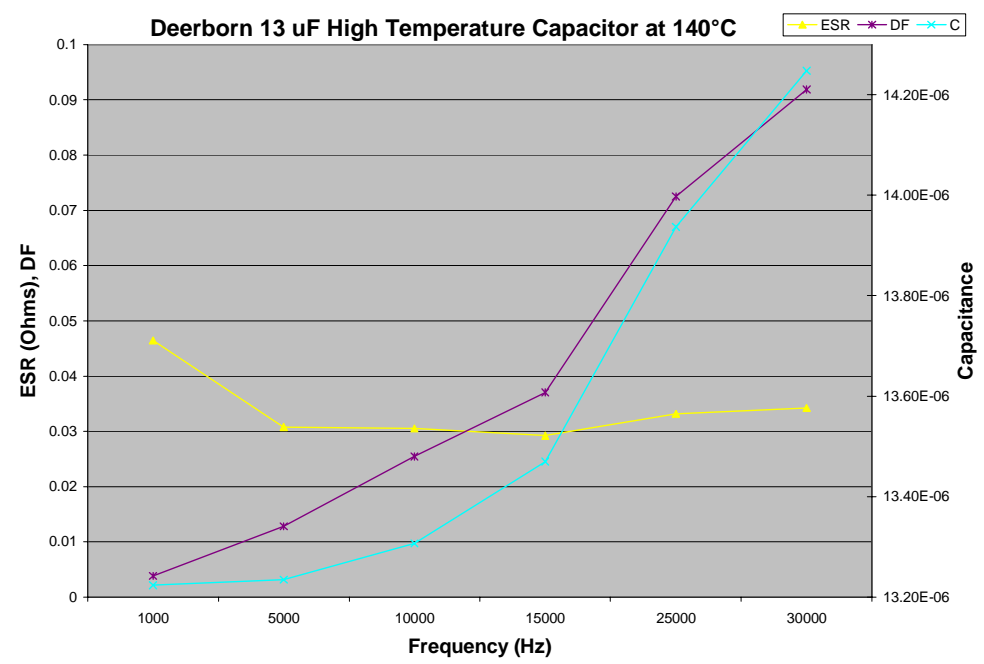

Figure 8. Dearborn metallized proprietary film capacitor at $140^{\circ} \mathrm{C}$.

\section{Semikron capacitor module (electronic concepts)}

The Semikron capacitor bank is $1000 \mu \mathrm{F}$ and consists of sixteen $62.5-\mu \mathrm{F}, 900-\mathrm{Vdc}$ capacitors in parallel. The capacitors are metallized polypropylene with a polyester wrap and epoxy end fill. The capacitor module was being tested at this writing.

\section{ESR Thermal Evaluation}

In an effort to accurately evaluate the data from these capacitor components, an independent method of measuring ESR is being developed. These tests are designed to validate the ESR for capacitors by quantifying the amount of heat released from the capacitor to its environment. A test stand has been developed consisting of a thermal chamber filled with oil, thermometers to monitor temperatures, a stirring method to keep the oil bath mixed, and insulation to maintain the temperature inside the container.

The test is accomplished by measuring the mass of the oil bath, capturing the initial conditions for the oil (specific heat, temperatures), and operating the test specimen for a period of time. The bath heats up as 
a result of the test specimen's waste heat. This waste heat in joules is calculated and related to the test time length, allowing the heat loss rate to be calculated.

Figure 9 shows the test setup. A LabView virtual instrument program has been written to capture all the critical data during the extended test period.

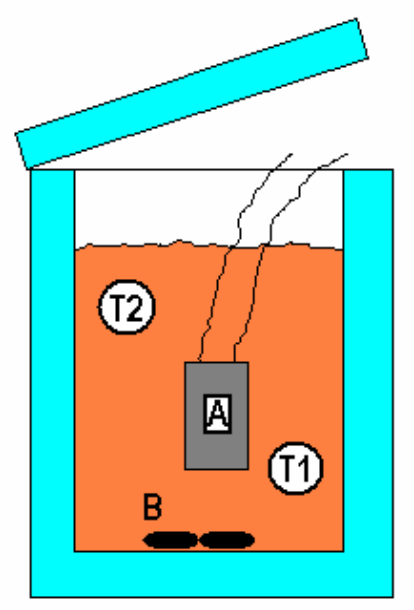

Insulated Box

Oil bath

\section{A- Test Specimen \\ B - Magnetic stir paddle}

T1, T2 - Temperature Sensors

Figure 9. ESR validation oil bath test setup.

The heat captured in the oil bath is calculated using the following relationship:

$$
\mathrm{Q}=\mathrm{m}_{\mathrm{oil}} * \mathrm{C}_{\mathrm{p}} * \Delta \text { temp }
$$

where

$\mathrm{Q}=$ Heat captured in the oil

$\mathrm{m}_{\mathrm{oil}}=$ mass of the test oil in the bath

$\mathrm{C}_{\mathrm{p}}=$ specific heat of the oil

$\Delta$ temp $=$ change in temperature of the oil $\left({ }^{\circ} \mathrm{C}\right)$ during the test

The bath is being calibrated by supplying a known amount of current into a known resistive load, measuring the voltage drop across the test resistor, and calculating the power in watts. The program inputs are then calibrated to produce sound thermal measurements. The thermal results can then be compared to the capacitor's electrical data, including ESR, to show that the electrical parameters measured (which would result in a certain amount of heat) match what is measured in the thermal bath.

\section{Magnet Evaluation}

Three types of new magnet technologies were evaluated and are reported here. Ames Laboratory provided a sample of 2205 MQP-O in a PPS binder; Argonne National Laboratory (ANL) provided a sintered NdFeB magnet; and Shin-Etsu Magnetics Inc. provided two samples of NdFeB (N43TS and $\mathrm{N} 41 \mathrm{TU})$. The magnet evaluations reported here are not at elevated temperatures.

The Ames and Argonne magnets were tested using a Walker Scientific AMH-40 Automatic Hysteresisgraph (Figure 10). This computer-controlled system is used to test, analyze, and evaluate the hysteresis properties of hard and very hard magnetic materials. The system consists of a PC-based control consol; two computer-interfaced integrating flux meters; a gauss meter; B and B-H coil sets; two 7-in.diameter, variable-gap, water-cooled electromagnets with cobalt-iron tipped poles; and a 5-kW, 50-A programmable power supply. The 1200-lb electromagnets are capable of generating demagnetizing forces 


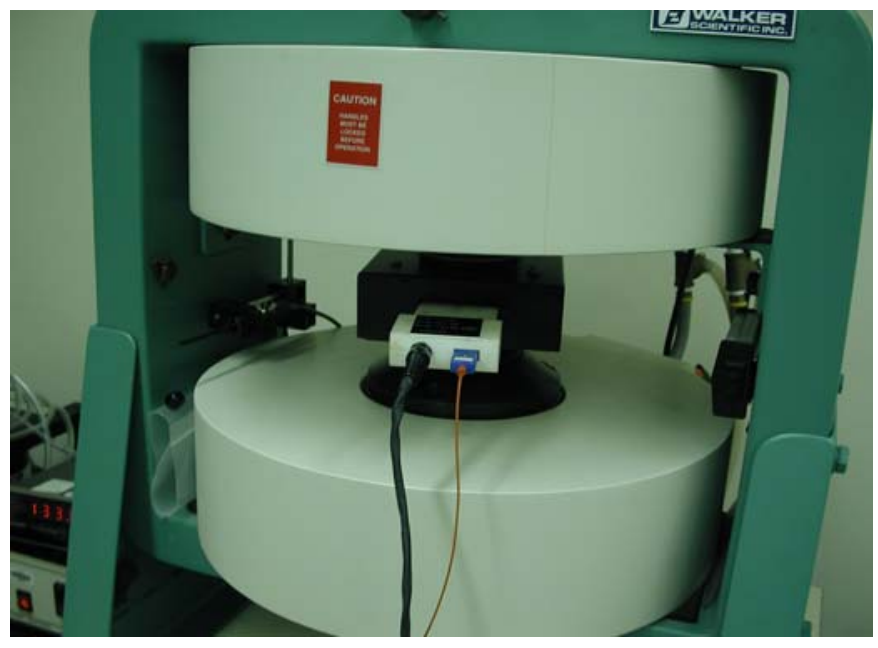

Figure 10. Walker Scientific AMH-40 Automatic Hysteresisgraph.

of up to 30,000 Orsteads. This system allows the operator to perform five different types of tests: manual $\mathrm{H}$-field sweep, second-quadrant demagnetization curves, complete four-quadrant full hysteresis loops, recoil minor loops after saturation in the first quadrant, and first-quadrant saturation or demagnetization of the samples. Also complementing the system is a pulse charger capable of saturating very hard magnetic materials and a Model TCS2 temperature-controlled water-cooled fixture for testing materials at temperatures ranging from -100 to $+300^{\circ} \mathrm{C}$. This fixture is used with a compatible thermocoupled hightemperature coil set.

\section{Ames Laboratory}

Two samples were tested from Ames Laboratory. The sample of 2205 bonded magnet material was tested using the Walker Hysteresisgraph. The de-magnetization curves were plotted at ambient temperature and are shown in Figure 11. The sample of 60\% MPQ-O and 40\% PPS bonded magnet material from Ames was also tested using the Hysteresisgraph. The de-magnetization curves were plotted at ambient temperature (Figure 12).

\section{Argonne National Laboratory}

A sample of $\mathrm{NdFeB}$ sintered magnet material was received from Dr. Cha at Argonne National Laboratory. This sample was tested in the Hysteresisgraph at ambient temperature. The hysteresis graph obtained from that test is shown in Figure 13.

\section{Shin-Etsu Magnetics}

Two samples of NdFeB (N43TS and N41TU) sintered magnets were obtained from Shin-Etsu Magnetics for testing on the Hysteresisgraph machine. These magnets were not magnetized when received. After the first hysteresis graph was conducted, it was apparent that the magnets were anisotropic across the diameter. This presented a problem in testing the magnet using the Hysteresisgraph machine. The geometry of the coil will not allow the magnet to be placed in the machine so that the magnetization and demagnetization process can produce the hysteresis graph. At this writing, the problem is being discussed with Shin-Etsu Magnetics. 


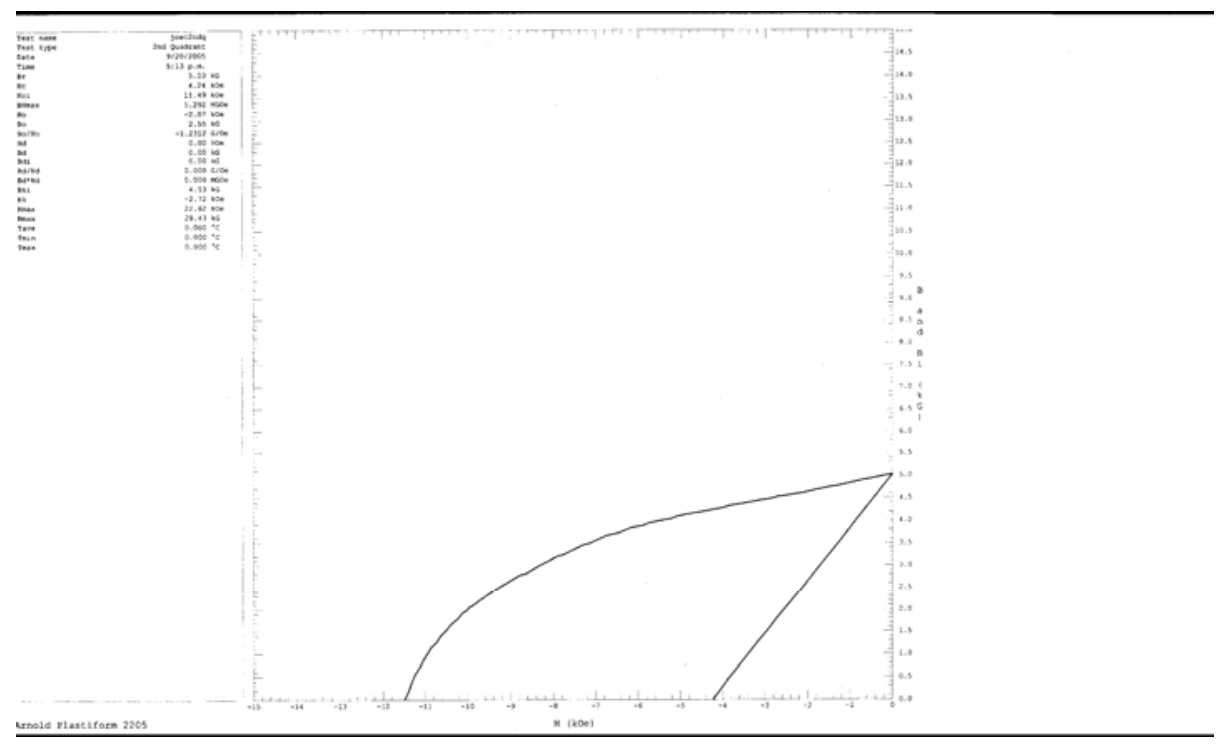

$\begin{array}{ll}\mathrm{Br} & 5.03 \mathrm{kG} \\ \mathrm{BHmax} & 5.29 \mathrm{MGOe} \\ \mathrm{HC} & 4.24 \mathrm{kOe} \\ \mathrm{HCi} & 11.49 \mathrm{kOe} \\ \mathrm{Hmax} & 22.62 \mathrm{kOe}\end{array}$

Figure 11. Ames 2205 bonded magnet demagnetization curves.

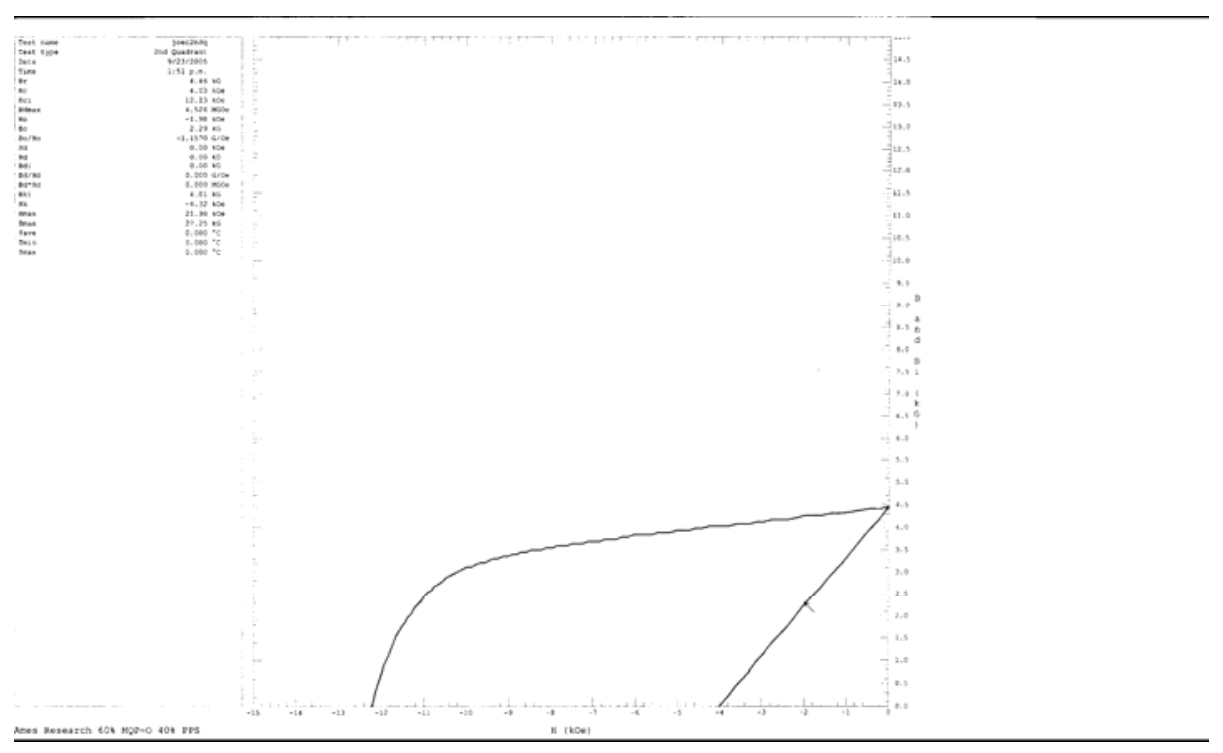

\begin{tabular}{lc}
$\mathrm{Br}$ & \multicolumn{1}{c}{$4.46 \mathrm{kG}$} \\
$\mathrm{BH} \max$ & $4.53 \mathrm{MGOe}$ \\
$\mathrm{Hc}$ & $4.03 \mathrm{kOe}$ \\
$\mathrm{Hci}$ & $12.23 \mathrm{kOe}$ \\
$\mathrm{Hmax}$ & $21.98 \mathrm{kOe}$ \\
Bmax & $27.25 \mathrm{kG}$
\end{tabular}

Figure 12. Ames 60\% MPQ-O and 40\% PPS bonded magnet demagnetization curves. 


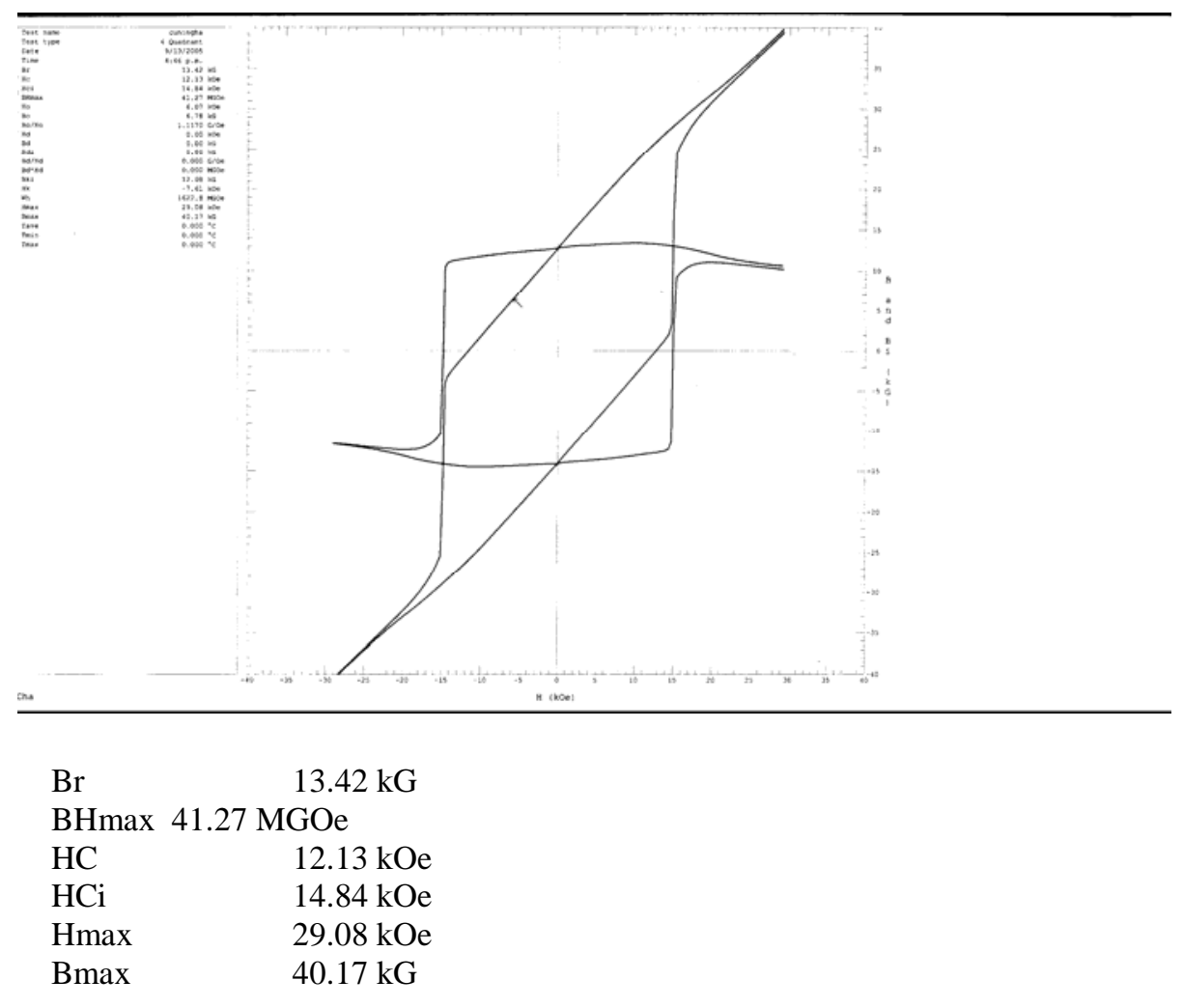

Figure 13. Argonne sintered magnet hysteresis graph.

\section{Future Direction}

The testing and characterization of magnets and capacitors will continue in FY 2006 as samples become available. Dynamic testing will be done on samples received in FY 2005. Temperature cycling and dynamic testing will be done on samples received in FY 2006. Thermal ESR evaluation will continue as an independent method of verifying ESR values that are questionable. Alternative methods of measuring ESL will also be explored. 


\title{
2.3 Benchmarking of Competitive Technologies
}

\author{
Principal Investigator: Robert Staunton \\ Oak Ridge National Laboratory \\ National Transportation Research Center \\ 2360 Cherahala Boulevard \\ Knoxville, TN 37932 \\ Voice:865-946-1351 ; Fax:865-946-1262 ; E-mail:stauntonrh@ornl.gov \\ DOE Technology Development Manager: Susan A. Rogers \\ Voice: 202-586-8997; Fax: 202-586-1600; E-mail: Susan.Rogers@ee.doe.gov \\ ORNL Program Manager: Mitch Olszewski \\ Voice: 865-946-1350; Fax: 865-946-1262; E-mail: olszewskim@ornl.gov
}

\section{Objectives}

The Benchmarking of Competitive Technologies project conducts electrical and thermal studies of hybrid electric vehicle (HEV) systems most recently introduced into the automotive market by non-U.S. companies. This produces data on efficiency, torque, speed, and voltage-current relationships. The full inspection and characterization of the electric motors and inverters also defines their thermal system performance in terms of temperature and corresponding sustainable power levels. Testing of these systems helps to rapidly advance the domestic technology base relative to electric traction drive systems suitable for HEV applications.

The FY 2005 accomplishments have made it possible to combine test data with design, packaging, and fabrication assessments to enable analysts to determine how various commercial HEV traction systems, such as the Accord and the Prius, compare with system and program technology targets (peakpower-to-weight and volume ratios).

\section{Approach}

As domestic vehicle manufacturers develop HEV systems, they are conducting periodic assessments of the HEV market dynamics, competitive HEV products, and production cost estimates for their own near-term products. Crucial information for the assessments can be obtained only through detailed evaluations of competitive HEV systems. Oak Ridge National Laboratory's (ORNL's) proposed testing program will reveal how the HEV systems contribute to vehicle performance, how the systems are manufactured and packaged, and what performance limitations exist.

Benchmarking of HEV traction drive systems provides detailed electrical and mechanical data from known permanent magnet synchronous motors (PMSMs) and inverters and yields information concerning their thermal performance. The ORNL testing program reveals how the systems are manufactured and packaged and what performance limitations exist. Data were obtained by instrumenting the HEV motor/inverter system and conducting locked-rotor, back-electromotive force (emf), mechanical and electrical loss, and broad-based thermal and performance tests over the entire design envelope. In essence, the overall approach of this project was to thoroughly define the systems and then perform a detailed evaluation of their performance in a controlled laboratory environment. This approach proved to be technically sound and successful.

Other facets of ORNL's technical approach include the following tasks:

- Provide the auto company partners with a consistent, open-literature source for complete characterization of HEV technology recently introduced in the marketplace. 
- Continue to use benchmarking information to assist in program planning, developing and executing specific projects, and supporting the partnership with original equipment manufacturers at the U.S. Council for Automotive Research.

- Confirm the realism of performance goals for the technologies and components and identify technology gaps.

- Query DOE and industry partners regarding emerging technology issues and the competitive technologies that hold the greatest technical interest.

- Use benchmarking data to validate multi-project R\&D modeling efforts, thus confirming that the validated modeling methods can be used appropriately to develop new technologies.

- Confirm the validity of the project technology thrust and avoid duplication of existing efforts.

- Based on resources, complete limited benchmarking of emerging hybrids or other subassembles and components selected by DOE, EE/TT, and/or industry partners.

- Provide technical insights that can be used to guide research efforts.

From ORNL's own technical perspective, there is an ongoing need for detailed electrical and mechanical data from known PMSMs and inverter designs to feed into analytical models and to support parametric studies. Data from successfully marketed HEV system designs are of special interest. These data can be obtained by instrumenting the HEV motor/inverter system and conducting locked-rotor, back-emf, loss, and broad-based thermal, and performance tests over the entire design envelope. The results of these tests will be shared with other ORNL programs, documented, and transmitted to $\mathrm{DOE}$ and domestic vehicle manufacturers on the EE/TT. The motor controller that was developed during the course of this project will be a valuable tool for future ORNL projects.

\section{Controller Development}

In order to operate the Prius motor outside the vehicle, a controller system had to be developed. The following provide examples of the technical hurdles and accomplishments related to this effort:

$\quad$ Since differentiating the
position signal produced spiking, the
approach was modified to measure
the frequency of the sinusoidal signal
from the resolver-with improved
results. It was also necessary to
reduce noise in the current,
frequency, and position feedback
signals. $\quad$ Because of a glitch in
Opal-RT program, controller
developers began to perform the
triangle-to-sine-wave comparison in
an external circuit; this made a
dramatic improvement in the stability
of the motor speed.
$\quad$ Programmers introduced
an enhanced model for motor/inverter
control that considers three ranges of
speed based on actual operating
constraints-each range is rigorously
defined by comprehensive equations
that are designed to maximize torque
per current.
$\quad$ A new code for a
motor/inverter controller was
completed and verified against a
motor simulation with excellent
agreement. (However, the model did
not have acceptable agreement with
the locked-rotor testing data, likely
...

\section{Major Accomplishments} order:

The following summarizes the main accomplishments for FY 2005 in approximate chronological

- The Prius motor was prepared for testing on the dynamometer. To ensure full motor loading capability, machining was required to attain direct coupling to the motor (bypassing the axle gears).

- The report Evaluation of 2004 Toyota Prius Hybrid Electric Drive System Interim Report, ORNL/TN-2004/247, was issued early in December.

- Partially funded by the benchmarking project, fixed-speed thermal testing of the Prius motor (using the dynamometer) was completed early in November. The data proved to be of high quality and were documented in Report on Toyota Prius Motor Thermal Management, ORNL/TM-2005/33, issued in February 2005.

- ORNL completed an analysis of Prius motor and inverter packaging, which provided estimates of peak-power-to-weight and -volume ratios 
- The code for a motor/inverter controller (see the text box) was completed in July, and it successfully ran the Prius motor to full speed and demonstrated full torque capability at all speeds. This allowed final preparation to begin for testing of the Prius system.

- ORNL completed an evaluation of Prius boost converter design and packaging.

- The data acquisition system was enhanced to write data from the Yokogawa PZ4000 power meter and numerous other sources to a single spreadsheet.

- ORNL began final performance testing of the Prius in July and August that led to the identification of hardware and EMI-related issues that were resolved in a timely fashion (see Technical Discussion).

- All required hybrid Accord HEV drive system components/assemblies were purchased during June/July; other parts required a 3-dimensional design process and fabrication (in FY 2006).

Much of the year was devoted to various aspects of preparing the test cell for comprehensive testing under controlled and verified conditions. This included ensuring (1) versatile control of the inverter/PMSM system, (2) precise electrical measurements amid high levels of EMI, (3) calibration of all critical instruments, and (4) the acquisition of approximately 50 channels of data. The test cell block diagram and corresponding layout are shown in Figure 1.
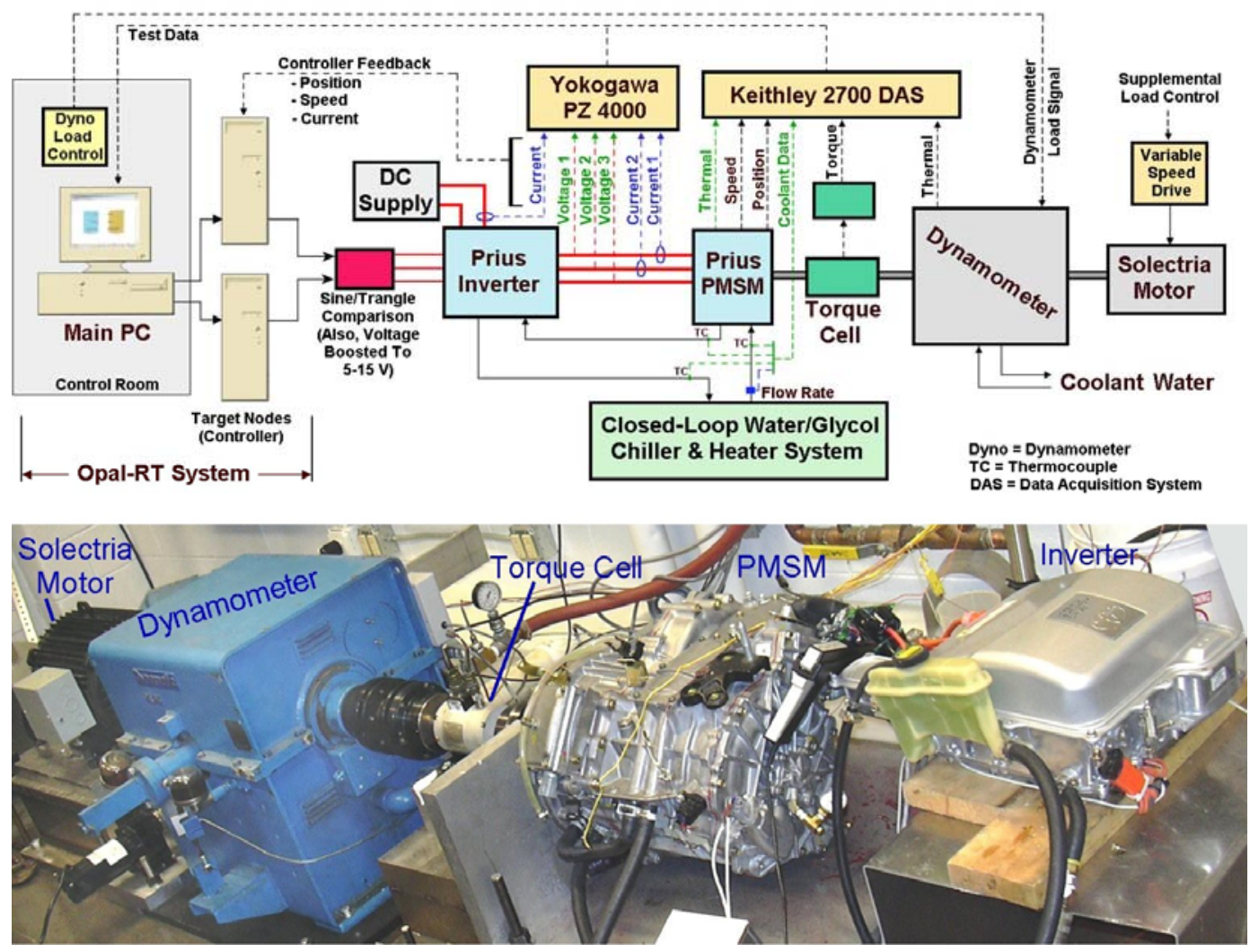

Figure 1. Test cell design, configuration, and actual layout.

\section{Technical Discussion}

The performance benchmarking of the 2004 Prius PMSM and inverter systems was accomplished through subassembly inspections/evaluations, a review of manufacturing and packaging, controller development in preparation for full design envelope testing, and laboratory evaluations. 
Specifically, the subassembly-level performance testing involved

- Collecting back-emf voltage waveforms for both the PMSM and generator

- Performing locked-rotor tests at varying torque angles (over the range of 90 to $134^{\circ}$ )

- Determining gear, bearing, and other friction losses for various operating speeds and lubricating oil temperatures

- Thermal testing at fixed speed (see Thermal Control Studies)

- Mapping motor/inverter performance over the full speed and shaft loading ranges using 50 and $90^{\circ} \mathrm{C}$ motor/inverter coolant temperatures (in progress).

\section{Controller Algorithm}

The algorithm for the controller was divided into three speed regimes: (1) a low-speed regime where operation is limited by current, (2) a mid-speed regime where operation is limited by voltage and current, and (3) a high-speed regime where operation is limited by voltage. In each case, the code was designed to maximize torque per current in a salient PMSM over a wide speed range with an adjustable level of field weakening.

First, a model of a salient PMSM was set up and simulated in Simulink/Opal. This was developed to help in verifying the controller algorithm. The machine model in the direct/quadrature coordinate system, as indicated by $\mathrm{d}$ and $\mathrm{q}$ subscripts, is given by

$$
\begin{aligned}
L_{d} \frac{d i_{d}}{d t} & =-R_{S} i_{d}+n_{p} \omega L_{q} i_{q}+u_{d}, \\
L_{q} \frac{d i_{q}}{d t} & =-R_{S} i_{q}-n_{p} \omega L_{d} i_{d}-K_{m} n_{p} \omega+u_{q}, \\
J \frac{d \omega}{d t} & =n_{p} K_{m} i_{q}+n_{p}\left(L_{d}-L_{q}\right) i_{d} i_{q}-\tau_{L},
\end{aligned}
$$

where $R_{S}=$ stator resistance, $n_{p}=$ number of pole pairs, $\omega=$ rotational speed $(\mathrm{rad} / \mathrm{s}), L=$ inductance, $J=$ the energy in joules, $i=$ current, $u=$ voltage, $K_{m}=$ the torque constant in the dq frame, and $\tau_{L}=$ load torque.

The field weakening problem is to maximize the torque, that is,

$$
n_{p} K_{m} i_{q}+n_{p}\left(L_{d}-L_{q}\right) i_{d} i_{q}
$$

subject to the following constraints:

$$
\begin{aligned}
V & =\sqrt{u_{d}^{2}+u_{q}^{2}} \leq V_{\max } . \\
I & =\sqrt{i_{d}^{2}+i_{q}^{2}} \leq I_{\max } .
\end{aligned}
$$

A controller algorithm was developed that, for any fixed speed, $\omega$, derives the values of $i_{d}(\omega), i_{q}(\omega)$, $u_{d}(\omega)$, and $u_{q}(\omega)$ that maximize torque for motoring, and minimize the torque for braking.

This is broken down into three cases: 
1.

$\sqrt{i_{d}^{2}+i_{q}^{2}}=I_{\max }, \sqrt{u_{d}^{2}+u_{q}^{2}} \leq V_{\max }$

2.

$\sqrt{i_{d}^{2}+i_{q}^{2}} \leq I_{\max }, \sqrt{u_{d}^{2}+u_{q}^{2}}=V_{\max }$

3.

$$
\sqrt{i_{d}^{2}+i_{q}^{2}}=I_{\max }, \sqrt{u_{d}^{2}+u_{q}^{2}}=V_{\max }
$$

This was further developed in a Matlab ${ }^{\mathrm{TM}}$ program that solves a static optimization problem. The control algorithm was used on the simulated PMSM with excellent agreement. This essentially verified the control algorithm. The next step was to validate the simulation model with data from the Prius machine.

Having a very high power density, the Prius motor operates well into the saturation régime. This has added complexity to the motor modeling and controller development efforts. Furthermore, the agreement between the model and motor operation data has not been as good as expected. Recent research papers $[1,2]$ indicate that the flux linkage in the $q$ axis depends on both the $d$ and $q$ currents. This also appears true for the $d$ axis flux linkage. Late in the year, the model was being revised accordingly so that it corresponds better with flux saturation levels and the locked-rotor data. This is expected to lead to an improved understanding of how an interior permanent magnet (IPM) motor such as the Prius should be modeled.

\section{Controller Hardware}

The RT-LAB real-time computing platform from OPAL-RT Technologies was used to model and replace the Prius control system. The RT-LAB system interfaces with the MATLAB SIMULINK software for quick controller development without tedious programming. The system consists of a host PC running a user-selected operating system and two target PCs running the QNX Neutrino operating system. One of the PCs is a dual-processor computer with additional counter, encoder, and analog/digital I/O PCI boards.

The model of the Prius controller and a user interface was built in MATLAB SIMULINK using both built-in SIMULINK blocks and RT-LAB blocks, as illustrated in Figure 2. This figure is an upper-level representation; most blocks can be opened for additional levels of detail. Using the Real-Time Workshop Toolbox of SIMULINK, the model was converted to C-source code and the executable uploaded to the target PCs. The controller software runs on two target PCs that communicate with each other through a Firewire connection, while the host computer is used to command the controller through an ethernet connection. The software allows the controller development to be flexible and versatile with the capability of quickly making required development changes. This approach bypasses some of the more difficult hardware development efforts required to allow the inverter to be controlled outside the vehicle. It also enhances the ability to make changes during testing, if required.

\section{dc Converter Assessment}

With planning under way for detailed performance testing at ORNL, it was necessary to review data that made clear the operation of the voltage boost circuit in the Prius. In addition, there was considerable technical interest in boost converters, including both their packaging and operation. In the Prius HEV system, the PMSM obtains most of its power from the generator and the rest from the high-voltagebattery-converter system. Thus the converter's power rating is less than half of the PMSM's rating. 


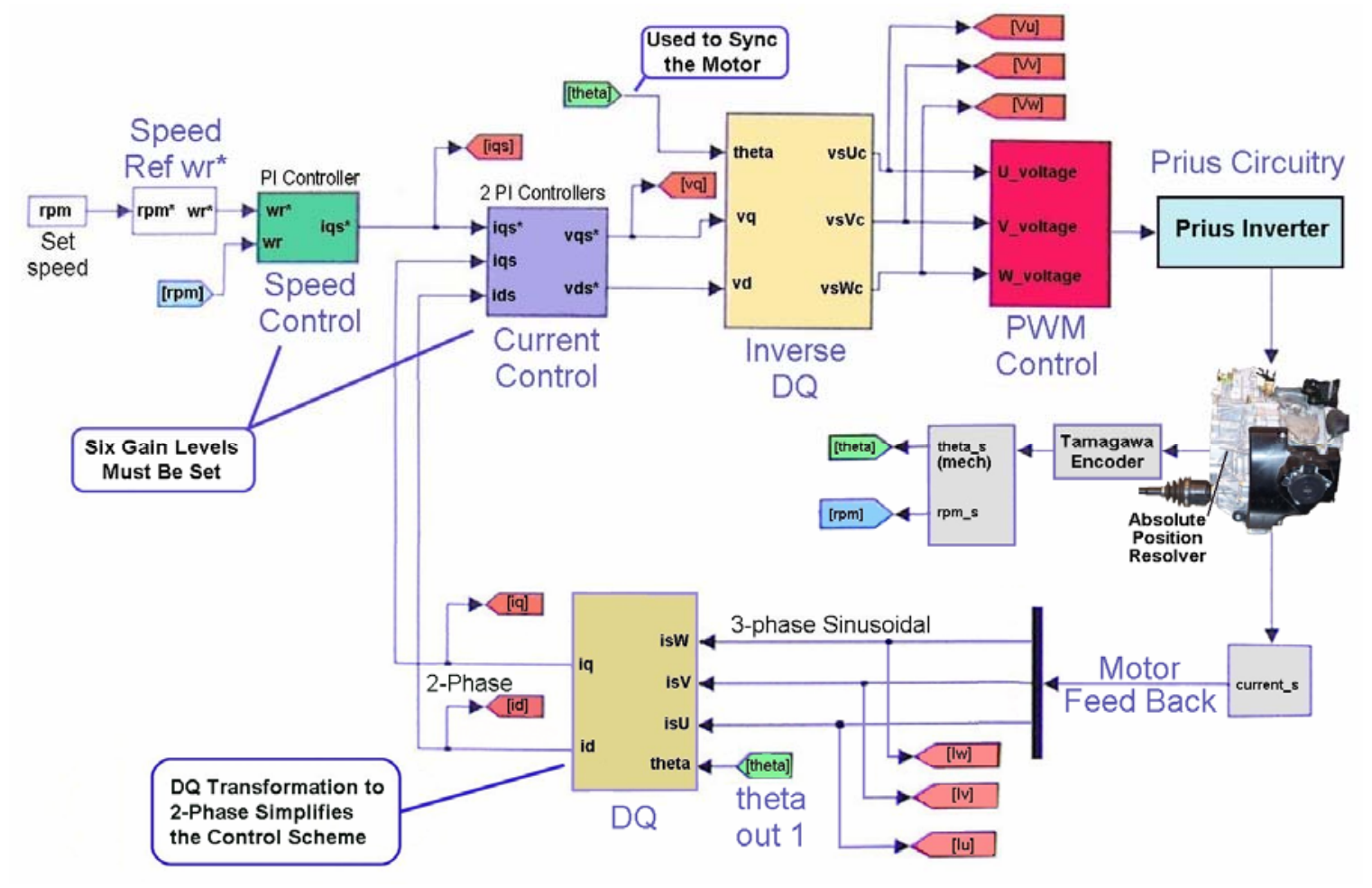

Figure 2. Upper-level flow diagram of Prius controller system.

The converter consists of three main components: an Intelligent Power Module, an auto-transformer, and a water-filled heat sink on which the first two are mounted. The module, simplified schematic, and transformer are shown in Figure 3. The figure indicates the mass and volume of the components and the total silicon area of the insulated gate bipolar transistors (IGBTs) and diodes. The boost converter is unusual in that two IGBTs are used instead of one so that two-way power flow can take place-the powering of the PMSM and the recharging of the high-voltage battery.

Of high interest is how the generator voltage, which increases with vehicle speed, compares with the voltage that the ECU directs the converter to produce. In order to determine this, the generator back-emf data plot was modified as shown in Figure 4 to show, by extrapolation, the voltage-speed relationship at speeds of up to $10,000 \mathrm{rpm}$. Also, the vehicle speed is included in the plot so that the data can be compared with vehicle operation data provided by Argonne National Laboratory (ANL). Note that the peak voltage developed in the generator is in the variable range of the dc converter when the vehicle is traveling 25-65 mph. This relationship will prove useful in the discussion below.

ANL generated vehicle drive cycle test data and, at ORNL's request, transmitted converter-related data to ORNL in April 2005. These data helped to characterize the operation of the voltage boost converter relative to acceleration and vehicle speed. 


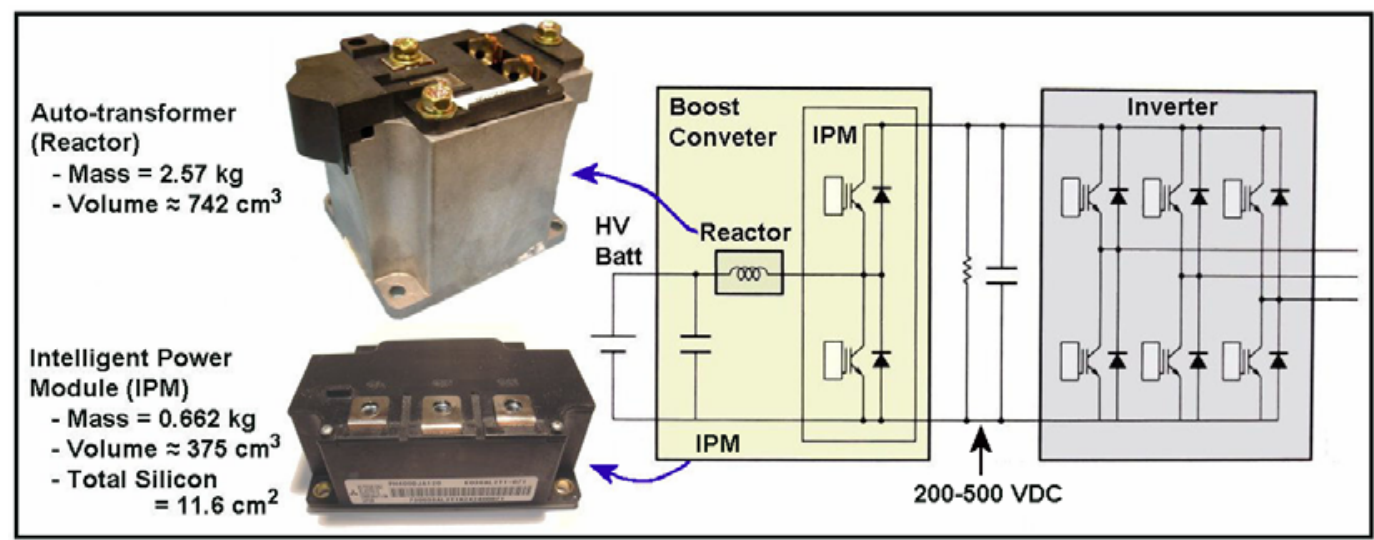

Figure 3. Voltage boost converter components and simplified schematic.

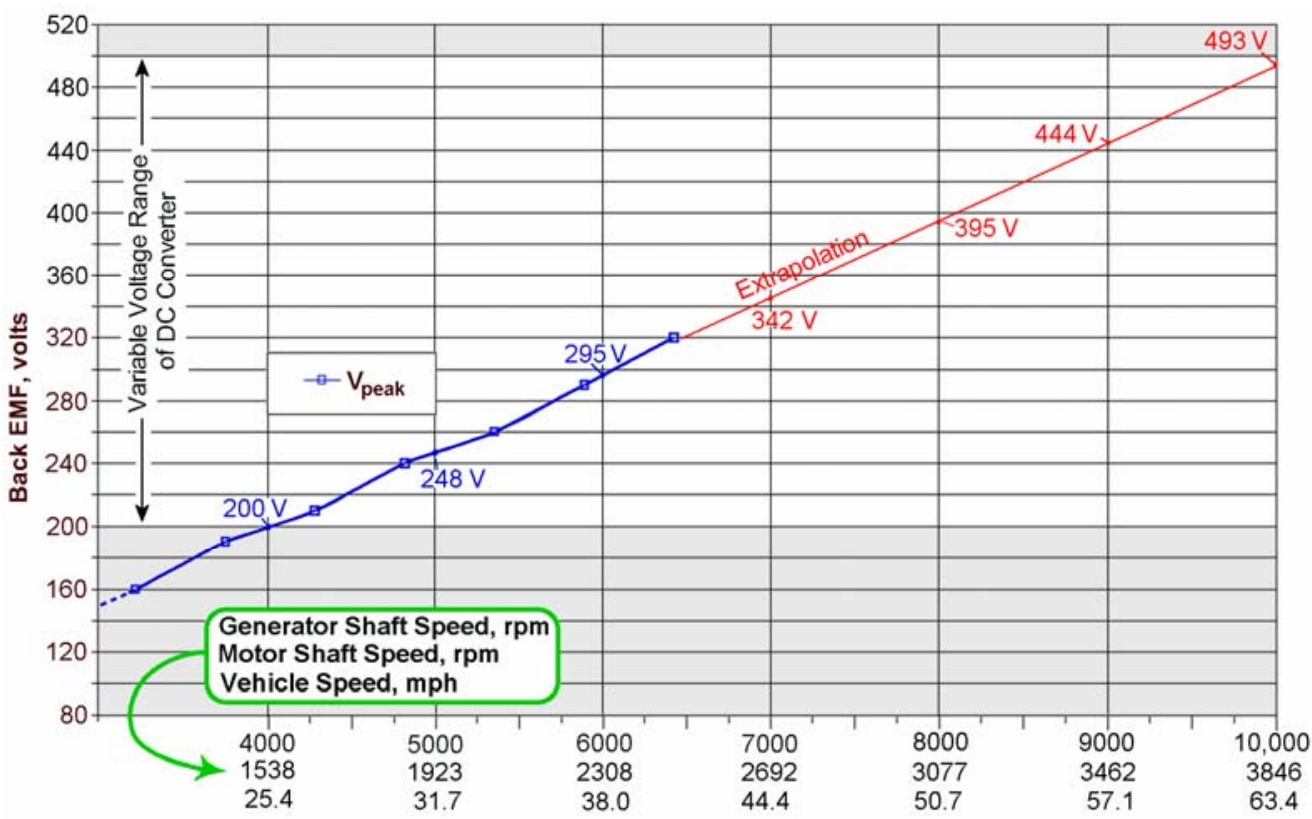

Figure 4. Extrapolation of generator back-emf data for generator speeds of up to $10,000 \mathrm{rpm}$.

Figure 5 shows data from a drive cycle where the vehicle was accelerated to about $31.5 \mathrm{mph}$, based on chassis-dyne data, followed by braking. The plot includes voltage and current output from the converter, accelerator pedal position output, brake pedal position output, and vehicle speed. A portion of the $y$-axis scale is expanded to enlarge/clarify the plots at the bottom of the chart (also making the depiction qualitative). As indicated in the figure, there are three instances where a current spike from the output of the voltage boost circuit preceded or accompanied a rise in the output voltage from the boost circuit. The voltage then remained elevated for different periods and then fell. Two instances occurred during acceleration and one at the end of the acceleration period. Note that braking causes a maximum boost to $500 \mathrm{~V}$ to support the regeneration battery-charging mode. The data do not appear to provide a full picture of what is happening; this is not surprising, since the ECU algorithm is unknown. 


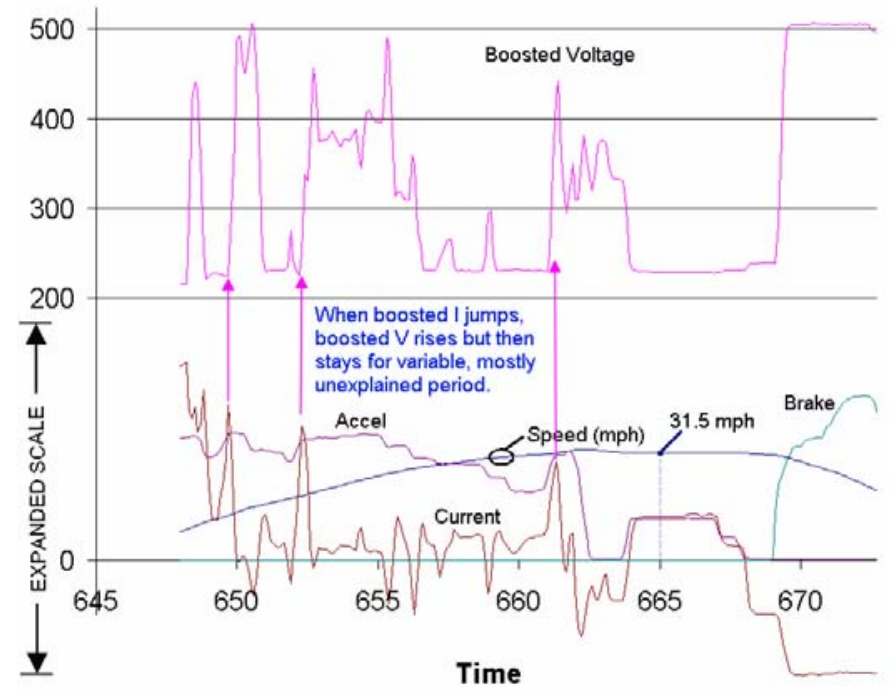

Figure 5. Drive cycle data from ANL showing voltage boost converter response.

The operation of the converter is further defined in the drive cycle plot in Figure 6. In this case, the vehicle is accelerated to about $68 \mathrm{mph}$. Because the level of acceleration is varied, the voltage boost swings between min and max several times. From $28.1 \mathrm{mph}$ to $49.8 \mathrm{mph}$, maximum voltage results from both the acceleration process and the increasing speed. Between $49.8 \mathrm{mph}$ and $59.7 \mathrm{mph}$, there is minimal tendency for voltage to drop below the max level. Above $59.7 \mathrm{mph}$, voltage remains at the max level. Notice that the plot also specifies the rotational speed of the $\mathrm{PMSM}^{1}$ at specified points.

Figure 6 also shows the peak back-emf from the generator based on ORNL testing as presented above. The points are plotted at the calculated equivalent vehicle speeds, and then approximate interconnecting lines are added for clarity. The data show how the boosted voltage rarely falls below the generator's peak voltage. Although the actual ECU algorithm for controlling the voltage boost is unknown, this plot and the preceding plot create a partial concept of how voltage is generally controlled during acceleration, high speeds, and braking. Because the rating of the converter prevents it from providing full power to the Prius motor, the converter was not used in performance characterization testing at ORNL.

\section{Subassembly Testing}

Motor and generator testing yielded back-emf voltage and frequency data. During the tests, a dynamometer drive was used to control motor speed and generator speed. Results of the back-emf testing are summarized in Table 1. These tests were repeated later in the year to verify that no measurable rotor demagnetization occurred as a result of prior thermal testing at elevated temperatures. It was found that the data agreed quite well (within instrument error).

\footnotetext{
${ }^{1}$ The rotational speed of the PMSM (rpm) is 60.66 times the vehicle speed in mph. This is based on the constant gear ratio of the Prius, wheel/tire diameter, and conversion of units.
} 


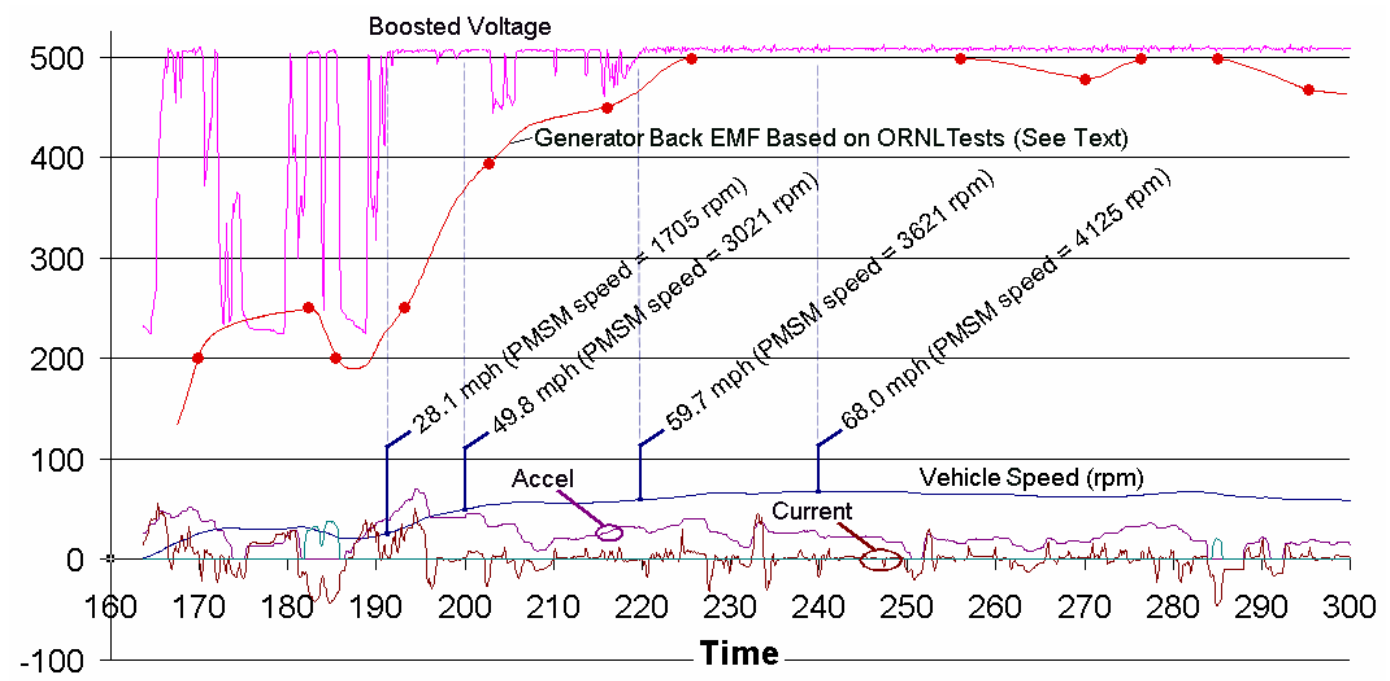

Figure 6. Drive cycle data set showing voltage boost at high speeds (ANL/ORNL data).

Table 1. Summary of back-emf test results

\begin{tabular}{lc}
\hline Test conditions & Results \\
\hline Motor back-emf $(6000 \mathrm{rpm})$ & $540 \mathrm{Vrms}$ \\
Motor Volts/Hertz & $1.33 \mathrm{Vrms} / \mathrm{Hz}$ \\
Generator back-emf $(6420 \mathrm{rpm})$ & $195 \mathrm{Vrms}$ \\
Generator Volts/Hertz & $0.46 \mathrm{Vrms} / \mathrm{Hz}$ \\
\hline
\end{tabular}

A series of locked-rotor tests was performed to determine the general operating capabilities of the traction motor. In particular, the torque and current were studied during the locked-rotor tests to characterize the startup torque capability of the motor. This test was repeated later in the year using improved methodology and equipment, since the prior data did not agree well with the Prius motor model. Figure 7 shows the torque vs rotor position plots for seven different current levels. Based on the plots, current and corresponding maximum torque values are summarized in Table 2 . The new data are more extensive and of higher quality; however, because the model does not fully account for magnetic saturation, some discrepancy remains. Finite-element analysis (FEA) studies are ongoing that may help to improve the model's handling of saturation.

\section{Motor Testing}

The benchmarking project partially supported and funded the thermal testing of the Prius motor. These tests were successful in determining the continuous ratings of the Prius motor design at coolant temperatures ranging from 35 to $105^{\circ} \mathrm{C}$. This information has not been released by the Toyota Motor Company. The ratings at base speed $(1200 \mathrm{rpm})$ were projected from test data at $900 \mathrm{rpm}$. The continuous ratings were determined to be $15 \mathrm{~kW}$ using $105^{\circ} \mathrm{C}$ coolant and $21 \mathrm{~kW}$ using $35^{\circ} \mathrm{C}$ coolant. These ratings are well below the $30-\mathrm{kW}$ target of the DOE FreedomCAR Program (see the Thermal Control Studies section for further discussion). 


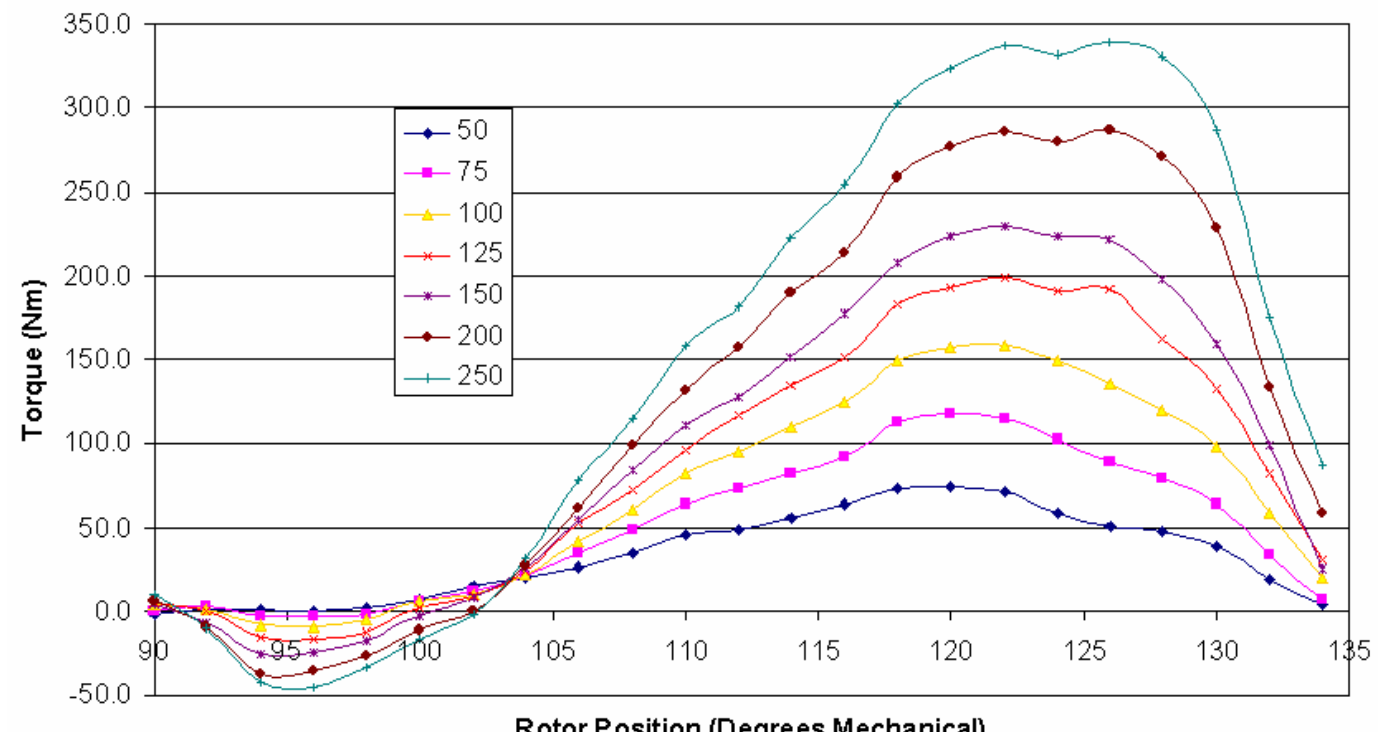

Rotor Position (Degrees Mechanical)

Figure 7. Prius locked-rotor data showing torque vs rotor position.

Table 2. Summary of motor current and torque test results

\begin{tabular}{cc}
\hline Current, A & Maximum torque, $\mathrm{Nm}$ \\
\hline 50 & 74 \\
75 & 118 \\
100 & 154 \\
125 & 199 \\
150 & 221 \\
200 & 286 \\
250 & 337 \\
\hline
\end{tabular}

The final testing of the Prius was the mapping of motor/inverter performance over the full speed and shaft loading ranges using 50 and $90^{\circ} \mathrm{C}$ motor/inverter coolant temperatures. Successive attempts at accomplishing this test during July and August were useful in identifying and ultimately resolving lingering barriers. In summary, excessive vibration was resolved by shaft realignment, a data-related discrepancy was resolved with a recalibration of the torque cell, and higher-than-expected temperatures led to a review of the test plan and modifications to allow safe testing at a higher temperature limit $\left(200^{\circ} \mathrm{C}\right)$. In the most recent test run, data showed unrealistically high $99 \%$ inverter efficiency (however, the error may be as small as one percentage point). This led to an investigation that ultimately pointed to EMI-induced noise introduced in the CT output circuitry as the most likely cause. Preliminary testing using fully shielded CTs indicated a significant improvement-a potentially complete resolution of this problem. Further testing in FY 2006 is required to verify these findings.

\section{Conclusion}

The Prius HEV system was fully evaluated by design inspection, disassembly, physical and electrical measurements, fabrication and packaging studies, and a series of laboratory spin and loss tests. In addition, thermal testing over a broad temperature range proved to be effective in determining the limitations of the continuous and peak power operation of the PMSM.

The importance of having developed a fully functional controller algorithm/code during the year cannot be overemphasized. ORNL now has a highly versatile tool that will enable laboratory testing of essentially any PMSM as long as position and speed sensors exist. The controller can adjust and explore 
varying levels of field weakening to determine the most efficient operating point. As shown in Figure 8, this controller system has already been put to use in another project to operate an experimental IPM motor even before completion of Prius testing. It is highly likely that this code will be used to operate a variety of motor designs for many years to come.

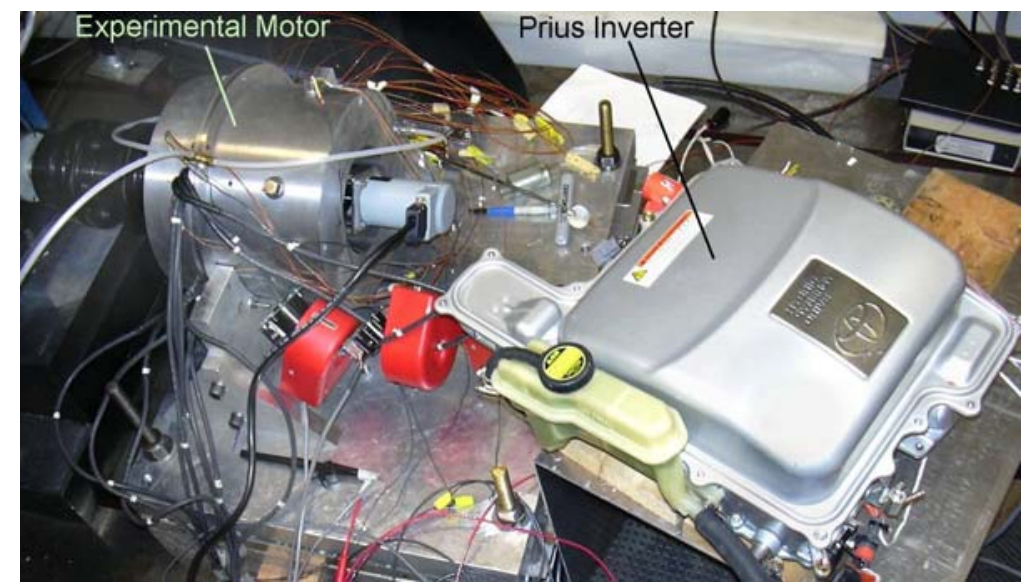

Figure 8. Prius controller/inverter system operating an IPM-reluctance motor with brushless field excitation.

It is estimated that 1-2 more weeks of testing in the test cell will produce all the data needed to map the entire speed vs torque range (full design envelope) of the Prius. Since the final Prius report draft is lacking only these data (and discussion), the data/report will be published early in FY 2006 and immediately disseminated.

\section{Future Direction}

During FY 2006, ORNL will perform benchmark testing of the hybrid Accord traction drive system. The test data and design, packaging, and fabrication assessment will be combined to enable analysts to determine how the Accord system compares with the Prius system and program technology targets (peakpower-to-weight and -volume ratios). Other hybrid systems will be considered for limited analysis based on resources and DOE/EETT interest. A decision will then be made by DOE in concert with the original equipment manufacturer partners regarding the need for further benchmarking.

Hybrid SUVs are emerging on the market and may represent significant deviations from existing hybrid technology. There may be some innovations in the drive system that merit benchmarking studies.

\section{Publications}

Evaluation of 2004 Toyota Prius Hybrid Electric Drive System Interim Report, ORNL/TM-2004/247, Oak Ridge National Laboratory, December 2004.

Report on Toyota Prius Motor Thermal Management, ORNL/TM-2005/33, Oak Ridge National Laboratory, February 2005

\section{References}

1. N. Bianchi, S. Bolognani, and B. J. Chalmers, "Salient-Rotor PM Synchronous Motors for an Extended Flux-Weakening Operation Range," IEEE Transactions on Industry Applications, 36(4) (July/August 2000).

2. S. D. Rubira and M. D. McCulloch, "Control Method Comparison of Doubly Fed Wind Generators Connected to the Grid by Asymmetric Transmission Lines," IEEE Transactions on Industry Applications, 36(4) (July/August 2000). 


\title{
3. Electric Machinery Research and Technology Development
}

\subsection{Field Weakening and CPSR Enhancement Techniques}

\author{
Principal Investigator: John W. McKeever \\ Oak Ridge National Laboratory \\ National Transportation Research Center \\ 2360 Cherahala Boulevard \\ Knoxville, TN 37932 \\ Voice: 865-946-1316; Fax: 865-946-1262; E-mail: mckeeverjw@ornl.gov \\ DOE Technology Development Manager: Susan A. Rogers \\ Voice: 202-586-8997; Fax: 202-586-1600; E-mail: Susan.Rogers@ee.doe.gov \\ ORNL Program Manager: Mitch Olszewski \\ Voice: 865-946-1350; Fax: 865-946-1262; E-mail: olszewskim@ornl.gov
}

\section{Objectives}

- To review, study, and evaluate recent progress in methods of flux control, both flux strengthening to increase torque at low speeds and flux weakening to extend the constant power speed range (CPSR). These features are being explored to extend motor design capability in order to develop motors that can meet torque/power/speed requirements without a gearbox. Success can result in a motor with higher power density (>10\%) at lower initial cost (80\%) than existing integral slot motors.

- To determine the conditions for maximum benefit using Oak Ridge National Laboratory's (ORNL's) dual mode inverter control (DMIC) to minimize current at high speeds under partial load and to quantify that benefit.

\section{Approach}

For this task, the research areas of field weakening and extending the CPSR were subdivided into

(1) voltage control, (2) flux control, (3) inductance control, (4) inverter control, and (5) reluctance control.

- Voltage control. As an example of benefits derived from voltage control, the Toyota Prius has cornered the HEV market with its THS II model by adding a boost converter to the earlier THS model, to deliver 50\% more power from the electric motor [1]. The increased voltage delivers more power with the original current so that the motor did not require redesign. The Z-source inverter [2] was developed to provide the voltage boost required by the fuel cell in an HEV. The Z-source inverter eliminates the shoot-through problem in transistors, which can destroy them in traditional inverters, and actually uses what formerly was dead time to boost the voltage. It makes better use of the inverter's pulse-width modulation (PWM) circuitry. The use of this inverter was explored and found to be inappropriate for an HEV powered by an internal combustion engine (ICE) because of the voltage drop across the diode. The same is true for its use with all electric vehicles.

- Flux control. Several methods of real-time flux control were explored, including traditional vector control [3], flux weakening in a consequent pole generator [4], flux control using an external coil [5], magnetic polarization variation (memory motors) [6], magnetic strength variation by thermal field weakening [7], actively switching the number of turns per coil while in operation [8], and gap control [9].

- Inductance control. Some earlier motor design experience implied that it is difficult to design sufficiently high inductance in integral-slot surface-mounted permanent magnet (SPM) motors to allow high-CPSR operation. Motor designers such as Toyota have focused their design on internal 
PM motors, which bury the magnet in the silicon steel rotor to increase their saliency [10]. ORNL teamed with the University of Wisconsin, Madison (UWM) to look at opportunities for PM synchronous motor (PMSM) cost reduction. Their report highlighted some significant benefits associated with fractional-slot PM motors with concentrated windings [11]. In particular, its inductance would be high because of the concentrated windings, and its structure could lead to material savings and ease of assembly. Because this design could achieve increased inductance along with other benefits, ORNL entered into collaboration with the University of Wisconsin to build and test a 6-kW fractional-slot PM motor with concentrated windings $[12,13]$. The measured inductance of the windings is as high as the analysis had predicted. The structure of the motors facilitated lowcost assembly with high slot utilization. ORNL has collaborated with UWM by modeling the 6-kW motor and by preparing preliminary models of a $30 / 55-\mathrm{kW}$ motor that meets FreedomCAR specifications. The notation 30/55 refers to continuous and intermittent power delivery. UWM has successfully tested the 6-kW motor to $4000 \mathrm{rpm}$. ORNL will receive the motor in FY 2005 to test a control scheme that promises a 7-8\% increase in efficiency at partial load [14]

- Inverter control. It is well known that the ability of the PMSM to operate over a wide CPSR depends upon the motor's inductance. Early approaches to extending the CPSR included adding inductances in each phase [15] and using anti-parallel thyristors in each phase [16]. The motor control method that requires increased inductance is compatible with a voltage source inverter controlled by PWM and is called conventional phase advance (CPA). The thyristor method is called DMIC. The fractional-slot motor with concentrated windings, which is being explored as part of this task, has the potential to make the PMSM motor compatible with CPA without external inductance and, if successful, will make the PMSM an attractive option for applications requiring high CPSR. The approach of this task was to model two PMSMs, one with the characteristic inductance that would allow operation at infinite CPSR and a second with a higher value of inductance equal to the value that was measured on the 6-kW fractional-slot motor with concentrated windings. Following this, integration of the motor model with models of the CPA and DMIC inverters was performed to explore their performance and find regions of performance where the cost-to-benefit ratio would justify the additional cost of the thyristors required by DMIC.

- Reluctance control. When vector control is applied to field-weaken a motor for operation at high CPSR the power and torque equations have two terms. The prominent term involves the interaction of the PM's flux linkages with the current in the coils and is the PM torque. A second term involves the difference between the stator inductance along an axis through the magnet, $\mathrm{L}_{\mathrm{d}}$, and the stator inductance along an axis displaced 90 electrical degrees, $\mathrm{L}_{\mathrm{q}}$, and is the reluctance torque. Rotors for which $\mathrm{L}_{\mathrm{q}} / \mathrm{L}_{\mathrm{d}}$ is greater than 1 are salient rotors, and the ratio is the saliency ratio. PMSMs with surface-mounted motors have uniform inductance around their periphery, so the saliency ratio is 1 , which means they are non-salient. Laboratory measurements have indicated that the reluctance torque for non-zero saliency motors can be significant. This task was initiated to see if additional torque could be obtained with a reluctance-assisted PM motor at low speeds while extending the CPSR without sacrificing performance. If it is successful, less magnet material would be required, which would lower the cost of the motor. The type of motor used in the study was an inset PM motor, which embeds the magnets in the rotor's silicon steel core so they are flush with the rotor surface. The magnet fraction is the ratio of the magnet pitch to the pole pitch. An early plot for a four-pole inset PM exhibited a magnet fraction at which the reluctance torque passed through a maximum, which was very close to where the total torque passed through a maximum. Encouraged by that result, this task was initiated to examine the performance response of the reluctance-assisted inset PM motor as the magnet fraction is varied. 


\section{Major Accomplishments}

\section{- Voltage Control}

- Reviewed the technology of boost converters with emphasis on the Z-source inverter, finding that it is excellent fuel cell vehicle (FCV) technology, whose present configuration does not migrate naturally into conventional ICE-powered HEV configurations.

— Presented the review and evaluation of the Z-source inverter in report ORNL/TM-2005/531.

- Flux Control

- Reviewed seven methods, two originating at ORNL and five presented in open literature, that may be used to increase the CPSR of PMSM motors while they are in operation without reducing their efficiency.

- Presented the results in report ORNL/TM-2005/175.

\section{- Inductance Control}

- Collaborated with UWM in designing 6-kW and 30/55-kW fractional-slot PMSMs with concentrated windings, which have potential to reduce material and assembly costs, increase slot utilization, and increase inductance for optimal CPSR.

- Built a 6-kW motor with concentrated windings and tested it at UWM to $4000 \mathrm{rpm}$.

- Compared analytical and measured results, which agreed reasonably well.

- UWM prepared preliminary 30/55-kW motor designs with fractional-slot concentrated windings.

- ORNL prepared preliminary 30/55-kW motor designs with integral slot windings, which will be compared with UMW's fractional-slot designs.

- Summarized and revised results in report ORNL/TM-2005/183.

\section{- Inverter Control}

- Developed an analytical per-phase fundamental frequency phasor model of a PMSM driven by CPA, including winding resistance and rotational losses.

- Developed an analytical per-phase fundamental frequency phasor model of a PMSM driven by DMIC, including winding resistance and rotational losses.

- Developed an analytical model for blocking losses and conduction losses in transistors, thyristors, and diodes; for switching losses in transistors; and for reverse recovery losses in diodes and thyristors.

- Affirmed the analytical model with a detailed PSPICE time domain simulation showing that results from the analytical model are sufficiently accurate for comparing the performance of different motor/inverter combinations.

- Applied the analytical model to two PMSMs, one with characteristic inductance, the lowest value that allows infinite CPSR, and one with the much higher inductance equal to the value measured on the 6-kW PMSM with fractional-slot concentrated windings to search for performance regions at partial load and to quantify the improved efficiency.

- Detailed results in report ORNL/TM-2005/184.

\section{- Reluctance Control}

- Completed a preliminary analysis to show the benefits derived from controlling reluctance in PMSMs.

- Applied SPEED software to analyze three configurations of interior PM motors (IPMs) to explore the impact on the performance of varying the magnet fraction. The configurations included an inset PM motor, an embedded V-shaped PM motor (type 4), and an embedded layered U-shaped PM motor (type 6).

- Applied the design equations of a reluctance motor to explore relations between variables and to develop a methodology for determining values of parameters that would result in optimum performance.

- The optimum number of turns per coil for maximum power delivery as a function of speed.

$\circ$ The optimum magnet fraction for power delivery as a function of speed.

- Summarized results in report ORNL/TM-2005-185. 


\section{$\underline{\text { Technical Discussion }}$}

\section{Voltage Control}

HEV are driven by at least two prime energy sources, such as an ICE and propulsion battery. For a series HEV configuration, the ICE drives only a generator, which maintains the state-of-charge (SOC) of propulsion and accessory batteries and drives the electric traction motor. For a parallel HEV configuration, the ICE is mechanically connected to directly drive the wheels as well as the generator, which likewise maintains the SOC of propulsion and accessory batteries and drives the electric traction motor. Today the prime energy source is an ICE; tomorrow it may be a fuel cell (FC). Use of the FC eliminates a direct-drive capability, accentuating the importance of the battery charge and discharge systems. In both systems, the electric traction motor may use the voltage directly from the batteries or from a boost converter that raises the voltage. If low battery voltage is used directly, some special control circuitry, such as DMIC, which adds a small cost, is necessary to drive the electric motor above base speed. If high voltage is chosen for more efficient motor operation or for high-speed operation, the propulsion battery voltage must be raised, which would require some type of two-quadrant bi-directional chopper with an additional cost.

Two common dc-to-dc converters are (1) the transformer-based boost or buck converter, which inverts a dc voltage, feeds the resulting ac into a transformer to raise or lower the voltage, and rectifies it to complete the conversion; and (2) the inductor-based switch-mode boost or buck converter [17]. A benefit of the transformer-based boost converter is that it isolates the high voltage from the low voltage. Usually the transformer is large, further increasing the cost. A useful feature of the switch-mode boost converter is its simplicity. Its inductor, which is responsible for its main cost, must handle the entire current. The new Z-source inverter technology $[18,19]$ boosts voltage directly by actively using the zero state time to boost the voltage. In the traditional PWM inverter, this time is used only to control the average voltage by disconnecting the supply voltage from the motor. The purpose of this study is to examine the Z-source's potential for reducing the cost and improving the reliability of FC HEVs.

The additional cost of a boost converter adds a significant cost penalty to the control system. Apparently this penalty has not discouraged the engineers at the Toyota Motor Corporation, because in September 2003 Toyota introduced a second generation of the Toyota Hybrid System (THS II), which boasted a 50\% improvement in motor power output [20], enabled by the use of a two-quadrant bidirectional chopper placed between the inverter's system voltage and the batteries' output voltage.

Recently, a new power converter topology was introduced that modifies a standard voltage-fed or current-fed PWM inverter and may be controlled to buck or boost not only dc-to-dc, but also dc-to-ac, acto-dc, and ac-to-ac. It is called a Z-source inverter [18, 19], and it adds a network of impedance to eliminate the problem of shoot-through. Shoot-through, which shoots the supply voltage through an inverter's upper and lower semiconductor switches, destroys the switches. It must be prevented by time delays in the control circuit. The Z-source inverter actually uses shoot-through to boost the voltage.

The research objective of the Z-source inverter was to provide a monolithic inverter to connect an FC with the traction drive of an HEV. The boost feature is essential for FC operation because the static characteristics of FCs exhibit more than a 30\% reduction in the output voltage between no-load and fullload current draw [21]. The buck feature in the opposite direction is needed because the storage batteries' SOC must be maintained.

Technology for the two traditional inverters, which are voltage-source inverters (VSIs) and currentsource inverters (CSIs), has been advanced by the invention of the Z-source inverter, which employs an impedance circuit to couple a power source to the input of an inverter [18, 19]. The Z-source inverter provides a power conversion process that may be used to synthesize waveforms with voltages above and below the source voltage. Its use eliminates the switch or switches required by a boost converter. It can be applied to all dc-to-ac, ac-to-dc, ac-to-ac, and dc-to-dc power conversions.

Traditional VSIs and CSIs have certain limitations not exhibited by the Z-source inverter. First, they may either boost or buck the voltage, but not both. Second, false gating, which can be induced by 
electromagnetic interference (EMI), may cause shoot-through in a VSI or may cause an open inductor circuit in a CSI, which will destroy the switches. Dead time for the VSI and overlap time for the CSI causes waveform distortion.

Figure 1 shows a schematic of the basic Z-source converter. If an FC is used, the blocking diode has the additional task of preventing current flow back into the FC in addition to its boosting function.

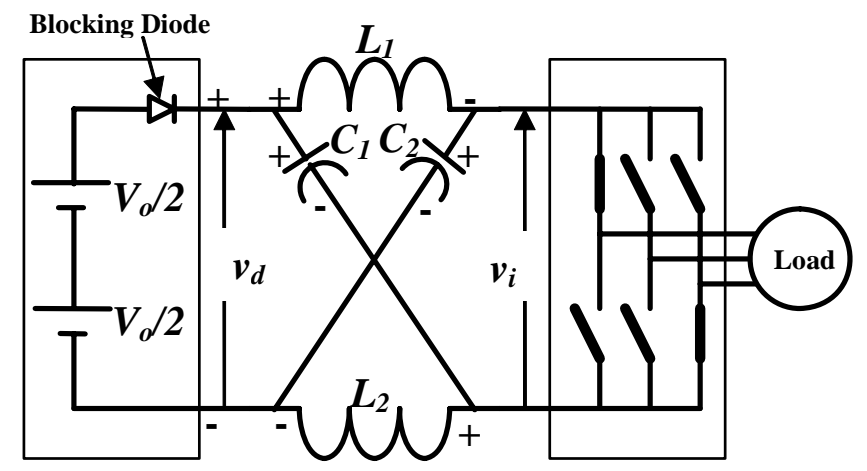

Figure 1. Field weakening and CPSR enhancement techniques.

As already mentioned, the Z-source inverter makes more complete use of the available operating time of a PWM inverter. During traditional PWM, an active semiconductor switch is turned off to disconnect the voltage source from the motor each time the magnitude of the triangular carrier voltage exceeds the magnitude of the modulating wave. It is not turned back on until the carrier wave falls back below the modulating wave. That time period is used by the traditional PWM inverter to synthesize the average voltage and may only allow the three upper switches or the three lower switches to be closed simultaneously to synthesize the average voltage. If an upper and lower switch in the same phase are closed, shoot-through occurs, which could destroy the switches in that phase. These two permitted states, one with three upper switches on or one with three lower switches on, are called zero states and all they do is create a path for the motor current when the supply is disconnected. Because of the two inductances and two capacitors in the Z-source circuit, shoot-through is permitted, allowing double use of what was formerly the zero vector time. This time is used not only to control the average voltage but also to boost the voltage.

There are two possible places to insert a propulsion battery in an FC-powered HEV driven by a Z-source inverter. The configuration shown in Figure 2 has been studied and reported [22]. In it, a battery is inserted in parallel with either one of the two Z-circuit capacitors because of circuit symmetry. The capacitor must remain across the battery terminals to absorb ripple currents, which shorten the battery life. Thus the battery must be a high-voltage battery whose voltage exceeds the FC voltage. The inverter module may be operated in the same way as traditional inverters to maintain the battery SOC with excess power from the FC and from regenerative braking. Furthermore, the high voltage across the propulsion battery is sufficient to operate the compressor motor expansion unit, thereby eliminating the dc-dc boost converter. 


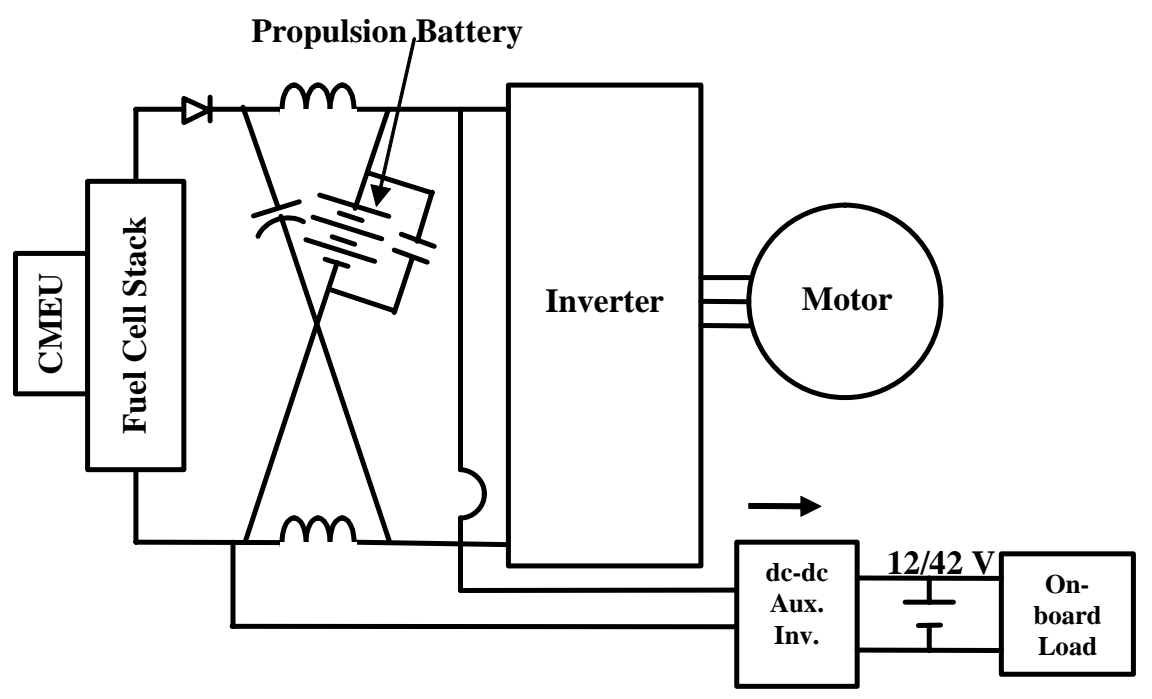

Figure 2. Configuration of a Z-source inverter-driven FCV.

Although the Z-source inverter was developed in the search for a better FC drive system, it poses questions when an attempt is made to apply its boost feature to a traditional ICE-powered HEV. Figure 3 shows one such configuration. Attempts to apply the Z-source inverter to an ICE-powered HEV revealed that Z-source technology does not migrate naturally into conventional ICE-powered HEV configurations. First, the FC is replaced by a low-voltage propulsion battery, which does not have the dramatic voltage drop exhibited by the FC as the load increases. Although the 200-V low-voltage propulsion battery in Figure 3 can provide a secondary source of power, it cannot receive regenerated braking power from the motor because of the intervening blocking diode required to isolate the $500-\mathrm{V}$ link from the $200-\mathrm{V}$ battery. In this configuration, the only available regenerated power to maintain the battery SOC must pass through the generator. Further, since the generator is connected to maintain the SOC on the secondary energy propulsion battery, it incurs additional losses when in drive mode because current must pass through the Z-source inverter's blocking diode. If the generator were connected directly to the highvoltage link, there would be no way to maintain the SOC on the propulsion battery, again because of the intervening diode. This might be accommodated by switching the generator connection between the lowvoltage and high-voltage buses, depending upon whether it is charging or driving; however, this adds a significant control complication and more hardware.

Further, as we explored the potential to replace the two-quadrant bi-directional chopper with the boost technology of a Z-source inverter, we found a straightforward modular replacement is complicated by the Z-circuit's integration requirements. The problem is that the connecting lines must be very short to prevent unacceptable inductance because the inductance cannot be easily compensated by snubber caps without affecting shoot-through operation of the Z-source inverter. Consequently, we conclude that, based on today's understanding, a new bi-directional configuration of the Z-source inverter will be required for use in ICE-powered HEVs.

One may compare the costs of the power build sections of each configuration. The power build section contains the semiconductors in the inverters that drive the motor and the generator; the semiconductors in the boost converter or in the chopper; and the inductances needed by the chopper, traditional boost converter, and Z-source inverter. These numbers are based on vendor quotes for 10001999 units for three levels of semiconductor packs [23]. Current in conventional PWM motor inverters and in traditional boost converters that feed PWM inverters requires 600-V/400-A dual packs at $\$ 269.60$ each. Current in the starter/generator inverter drives and in the PWM inverter fed by a traditional boost converter require only 600-V/200-A six packs at $\$ 240$ each. Current in the Z-source inverters employed $600-\mathrm{V} / 300-\mathrm{A}$ six packs at $\$ 308.88$ each. 


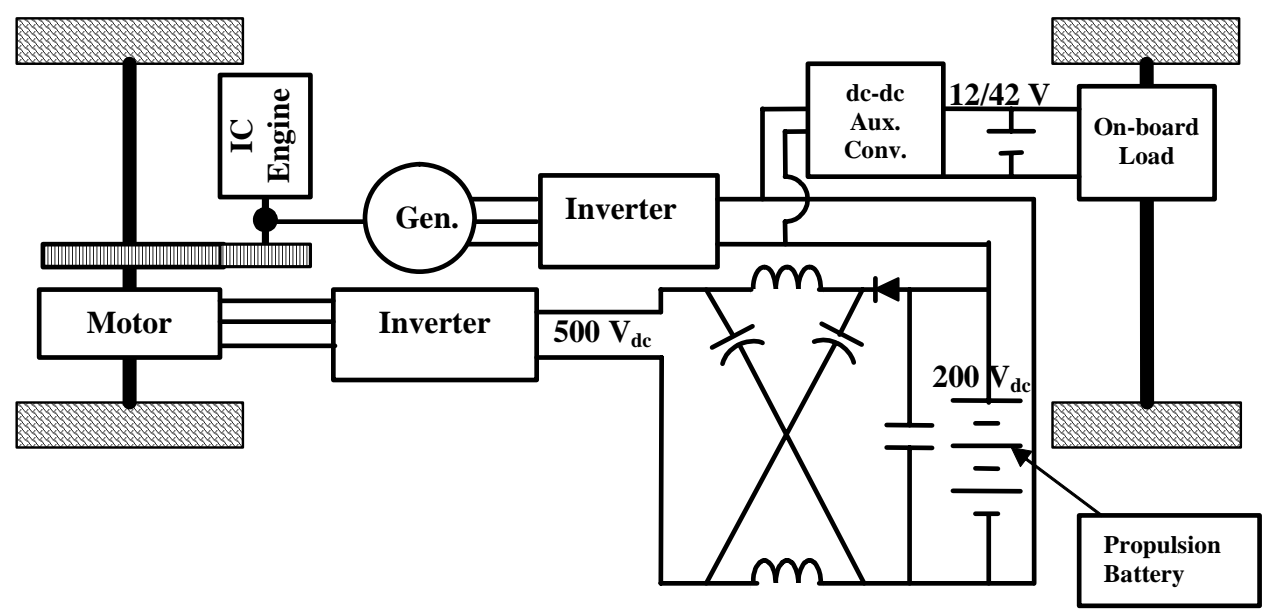

Figure 3. A configuration of an ICE-powered HEV driven by a Z-source inverter.

Now we shall look at some cost comparisons for different power build sections. There is general consensus among those who have studied the TH SII that it is the current baseline with the most desirable features. Its power build section estimated cost is $\$ 1392$. The power build section's estimated cost difference between THS I and THS II is $\$ 343$, which is an estimate of what Toyota was willing to pay to improve reliability and to deliver 50\% more power without changing the motor/drive size. What stands out is the drop in cost of the power build section when FCs replace the ICE as the primary energy source. The power build section of the FC-powered HEV with a traditional boost converter and a bi-directional chopper, which is comparable to Toyota's THS II in function, costs about $\$ 996$, which is $\$ 396$ less than the THS II. Most notable, however, is the dramatic reduction of the power build section cost to $\$ 383$ for the FC-powered Z-source inverter with a high-voltage battery as a secondary energy source in parallel with one of the two capacitors. This power build section cost is \$666 below the THS I cost and \$1009 below the THS II cost. Furthermore, it is \$201 less than the power build section cost of the conventional $\mathrm{FC}$ inverter, which is fed by a traditional boost converter.

The recent invention of the Z-source inverter provides a new technology that can eliminate the switch in the traditional boost converter between the FC and the high-voltage dc link. It can also eliminate the two additional switches between a moderate $200-\mathrm{V}$ secondary energy storage voltage and a high $500-\mathrm{V}$ traction motor voltage similar to that used in the THS II system. It eliminates all danger from shootthrough, which short circuits the supply voltage through the inverter switches, destroying switches in a traditional inverter. Instead, it makes double use of the zero-state time to boost the voltage as well as control the average voltage. Components introduced by the FC-powered Z-source inverter with a battery replacing one of the capacitors over the traditional boost converter are two small inductors to replace one large inductor with little cost difference. One less capacitor and one less switch are required.

The ICE or the FC must supply power to more than the traction motor. Hybrid ICEs and FCVs must supply power to charge a $12 / 42-\mathrm{V}$ auxiliary storage battery and a $200-\mathrm{V}$ or $500-\mathrm{V}$ secondary energy storage system. The energy storage system supplements the power from the ICE or FC, allowing the vehicle to deliver rated power during high-load conditions. If the Z-source inverter is applied to both hybrid ICEs and FCVs, the challenge is to connect it to the power source without interfering with its shoot-through boost operation. 


\section{Flux Control}

Significant research at the ORNL Power Electronics and Electric Machinery Research Center (PEEMRC) is being conducted to develop ways to increase (1) torque, (2) speed range, and (3) efficiency of traction electric motors for HEVs and FCVs within existing current and voltage bounds. Current is limited by the inverter semiconductor devices' capability; and voltage is limited by the stator wire insulation's ability to withstand the maximum back-electromotive force (emf), which occurs at the upper end of the speed range.

One research track has been to explore ways to control the path and magnitude of magnetic flux while the motor is operating. The phrase "real time flux control" (RTFC), refers to this mode of operation in which system parameters are changed while the motor is operating to improve its performance and speed range. RTFC has the potential to meet an increased torque demand by introducing additional flux through the main air gap from an external source. It can augment the speed range by diverting flux away from the main air gap to reduce back-emf at high speeds. Conventional RTFC technology is known as vector control $[3,24]$. Vector control decomposes the stator current into two components; one that produces torque and a second that opposes (weakens) the magnetic field generated by the rotor, thereby requiring more overall stator current and reducing the efficiency. Efficiency can be improved by selecting an RTFC method that reduces the back-emf without increasing the average current. This favors methods that use pulse currents or very low currents to achieve field weakening.

Foremost in ORNL's effort to develop flux control is the work of J. S. Hsu. Early research [4] introduced direct control of air-gap flux in PMSMs and demonstrated it with a flux-controlled generator. The configuration eliminates the problem of demagnetization because it diverts all the flux from the magnets instead of trying to oppose it. It is robust and could be particularly useful for PM generators and electric vehicle drives. Recent efforts have introduced a brushless machine that transfers a magnetomotive (mmf) force generated by a stationary excitation coil to the rotor [5]. Although a conventional PM machine may be field-weakened using vector control, the air-gap flux density cannot be effectively enhanced. In Hsu's new machine, the magnetic field generated by the rotor's PM may be augmented by the field from the stationery excitation coil and channeled with flux guides to its desired destination to enhance the air-gap flux that produces torque. The magnetic field can also be weakened by reversing the current in the stationary excitation winding. A patent for advanced technology in this area is pending.

Several additional RTFC methods have been discussed in open literature. These include methods of changing the number of poles by magnetizing and demagnetizing the magnets' poles with pulses of current corresponding to direct-axis (d-axis) current of vector control [6], changing the number of stator coils [8], and controlling the air gap [9]. Test experience has shown that the magnet strengths may vary and weaken naturally as rotor temperature increases, suggesting that careful control of the rotor temperature, which is no easy task, could yield another method of RTFC.

The purpose of this research was (1) to examine the interaction of rotor and stator flux with regard to RTFC, (2) to review and summarize the status of RTFC technology, and (3) to compare and evaluate methods for RTFC with respect to maturity, advantages and limitations, deployment difficulty, and relative complexity.

Most approaches to extend the speed range of operation of PM motors focus on weakening the rotor's magnetic flux in order to decrease its contribution to the magnitude of the back-emf and thus allow for power input and generation of stator magnetic field at higher speeds. This weakening may be accomplished in several ways, as discussed in the following paragraphs.

\section{Vector control}

The traditional approach to field weakening is vector control, in which the effective rotor magnetic flux is suppressed by controlling d-axis stator current to generate a magnetic field that directly opposes and thereby weakens each PM's field. When this approach is used, one must be careful not to demagnetize the PMs. If, in addition, higher torque must be maintained at high speed, extra quadrature- 
axis (q-axis) stator current must be supplied besides the current invested to suppress the rotor's magnetic field, which results in higher resistance losses and lower efficiency.

\section{Flux weakening in consequent pole generator}

Research at ORNL [4] has produced a consequent pole generator with an external coil connected to the stator. A schematic is shown in Figure 4. A consequent pole device replaces alternate magnetic poles with ferromagnetic poles and doubles the thickness of the remaining magnets. The alternate pole is induced in the ferromagnetic material as a consequence of the magnet. Current in the external coil controls flux, allowing the consequent pole to aid, to do nothing, or to oppose the magnet flux during generation of a back-emf. In Figure 4(b), flux in the external coil forces the magnet flux into the consequent pole to aid the magnet as it generates the largest back-emf. In Figure 4(c), the external-flux path accommodates the entire magnet flux, thus removing the consequent pole as the magnet generates a back-emf of its traditional equivalent. In Figure 4(d), the external path provides flux to oppose the magnet, thereby reducing the back-emf to a low value. The external coil requires a small amount of additional current, but it may be used with a conventional PWM inverter and it eliminates completely the danger of demagnetizing the magnets. The only increase in complexity is a controller for the auxiliary stator coil current.

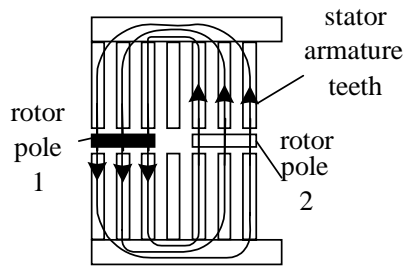

(a) Normal PM flux path

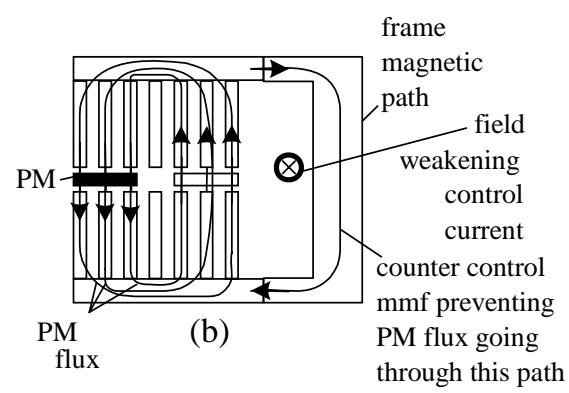

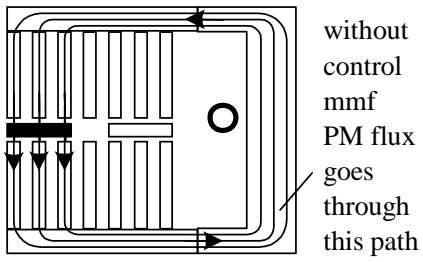

(c)

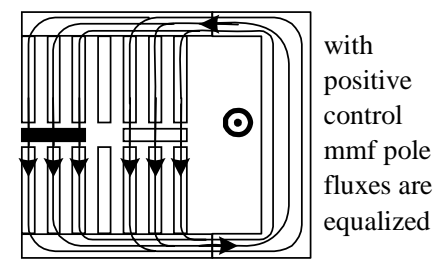

(d)

Figure 4. Axial-gap consequent pole generator weakened and strengthened by external coil.

\section{High-strength undiffused brushless PM motor.}

Another innovative approach at ORNL [5] employs an external-flux control coil that boosts the magnetic flux at low speeds and weakens it at high speeds. For a conventional PM motor, the air-gap flux density cannot be enhanced effectively, but it can be weakened; consequently, this approach addresses the problem of reduced back-emf at low speeds as well as the problem of excessive back-emf at high speeds. A schematic is shown in Figure 5.

The new machine is brushless because it transfers the mmf from the stationary excitation coil to the rotor through two secondary air gaps, identified as "air gap" in Figure 5. In the new machine, the PM in the rotor prevents magnetic-flux diffusion between the poles and guides the reluctance-flux path. The pole-flux density in the air gap can be higher than what the PM alone can produce, thus resulting in a machine with high magnetic strength. The higher magnetic strength manifests itself in higher torque. 


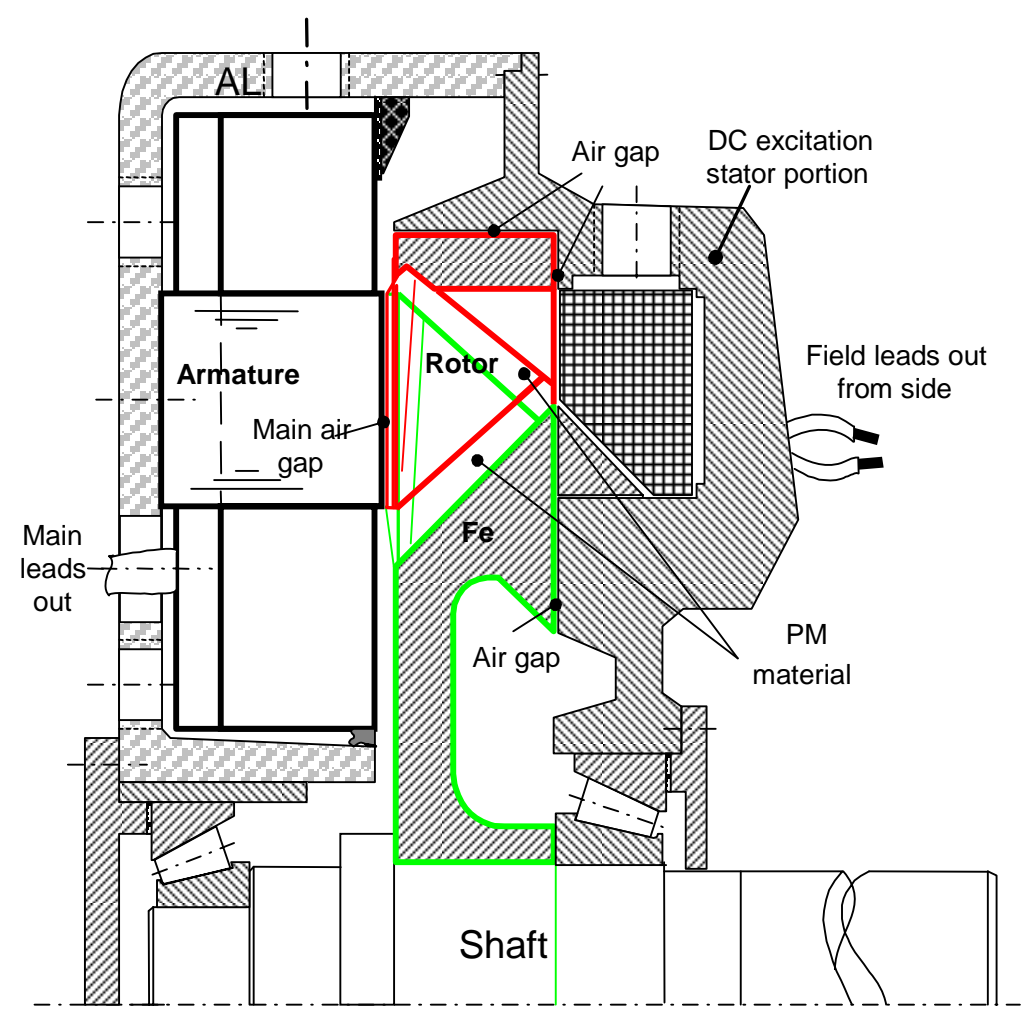

Figure 5. High-strength (magnetic) undiffused brushless axial-gap PM motor.

The axial flux produced by the dc excitation stator coil passes across the secondary air gap into the center part of the rotor (green), into the pole face (green), across the main air gap into a stator tooth, through the stator yoke to the adjacent tooth, back through the main gap into the rotor pole face (red), radially outward through the secondary air gap, and back through the external coil housing to close the flux path.

\section{Magnetic polarization variation-memory motors}

A fourth approach is to change the magnetization of the PMs by means of mmf pulses [6] so that the strength of the rotor poles is changed at will while the motor is operating. This type of motor is a variableflux motor. If the magnets are demagnetized completely, the result is to decrease the number of poles. This second type of motor is a pole-changing motor. Ostovic calls this motor a "memory motor" because of its ability to memorize the flux-density level in the rotor magnets.

Figure 6 is a schematic of the memory motor. The magnets are polarized circumferentially and placed in a consequent pole configuration. The magnetization of the PMs can be varied by a short-current pulse and does not require a steady demagnetization current characteristic of vector control; consequently, its efficiency is higher. The demagnetizing current flows through the stator windings, requiring no special current source. The memory motor combines the advantages of a wound rotor, with its variable flux, and a PM motor with no excitation losses, whose benefits suggest it may be a candidate for an HEV traction drive. 


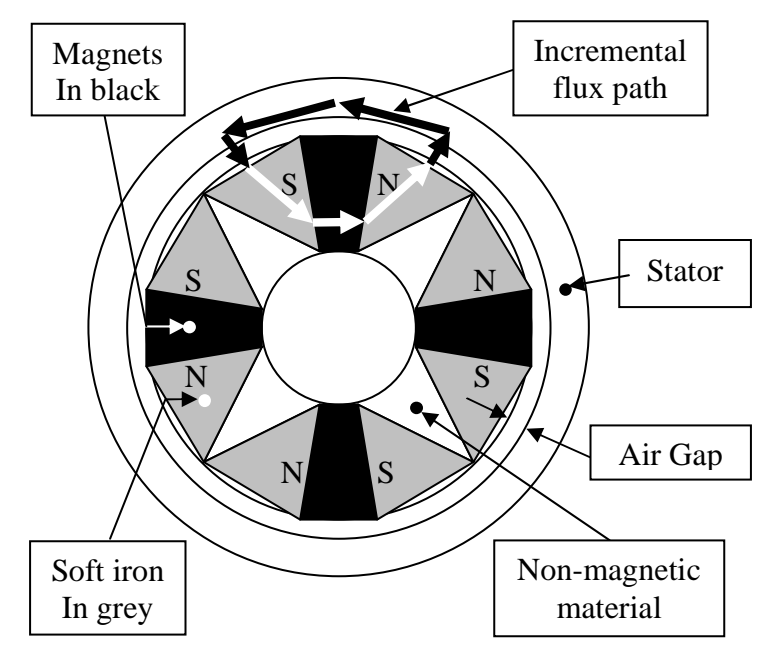

Figure 6. Variable flux PM motor-memory motor.

\section{Magnetic strength variation-thermal field weakening}

A fifth approach could be to control the strength of the PMs by actively or passively regulating their temperature below the material's maximum service temperature so that permanent demagnetization will not occur [7]. The effect of thermal field weakening has been observed during PM motor testing by a reduction in the field weakening current required to maintain operation as the speed increases. The total current is the square root of the d-axis (field weakening) current squared plus q-axis current squared. The reduction in total current observed during the test is a result of the lower d-axis current. The anticipated result is higher efficiency.

\section{Changing number of stator turns per coil}

Relationships between stator current and stator-magnetic flux, as well as between rotor flux and backemf, are usually considered fixed by construction; but this is an unnecessary constraint. A reduction in back-emf reduces the wasted current required to weaken the rotor magnet's field; consequently, another approach for RTFC is to decrease the back-emf during operation by reducing the number of turns per coil involved in the energy conversion.

Changing the number of turns per coil can be done by (1) physically changing the number of active turns, (2) changing the turn-to-flux link factor, or (3) changing the number of poles. We have already seen how the memory motor can change the number of poles by demagnetizing the magnets. Changing the number of turns may be accomplished by using winding taps with two rectifiers and two switches [8].

For illustration, the speed dependence of the maximum power output for a typical IPM motor with a hypothetical capability to change the number of turns at will is shown in the figures below. Figure 7(a) shows how the base speed, peak power, power, current, and speed ranges grow as the number of turns is reduced from 9 to 5. Figure 7(b) shows the limit power curves for a turn-switching scheme focused on maximum power generation.

We now discuss the methodology for maximizing power delivery with respect to the number of turns per coil. It is representative of the approach that will be used for optimization with respect to other motor parameters.

A reduction in the effective number of turns per coil, $N_{s}$, decreases the electrical inductance, $\mathrm{L}_{\mathrm{s}}=N_{s}{ }^{2}$ $\mathrm{L}_{1}$, and ohmic resistance, $\mathrm{R}=\mathrm{N}_{\mathrm{s}} \mathrm{R}_{1}$, where $\mathrm{L}_{1}$ and $\mathrm{R}_{1}$ are the inductance and resistance, respectively, of a coil comprising one single turn. This approach will result in higher currents for the same voltage levels and in enhanced efficiencies. The following discussion explains how the equation may be derived for the number of turns per coil that will deliver maximum power as a function of angular frequency. 
Power \& Current Vs. Speed

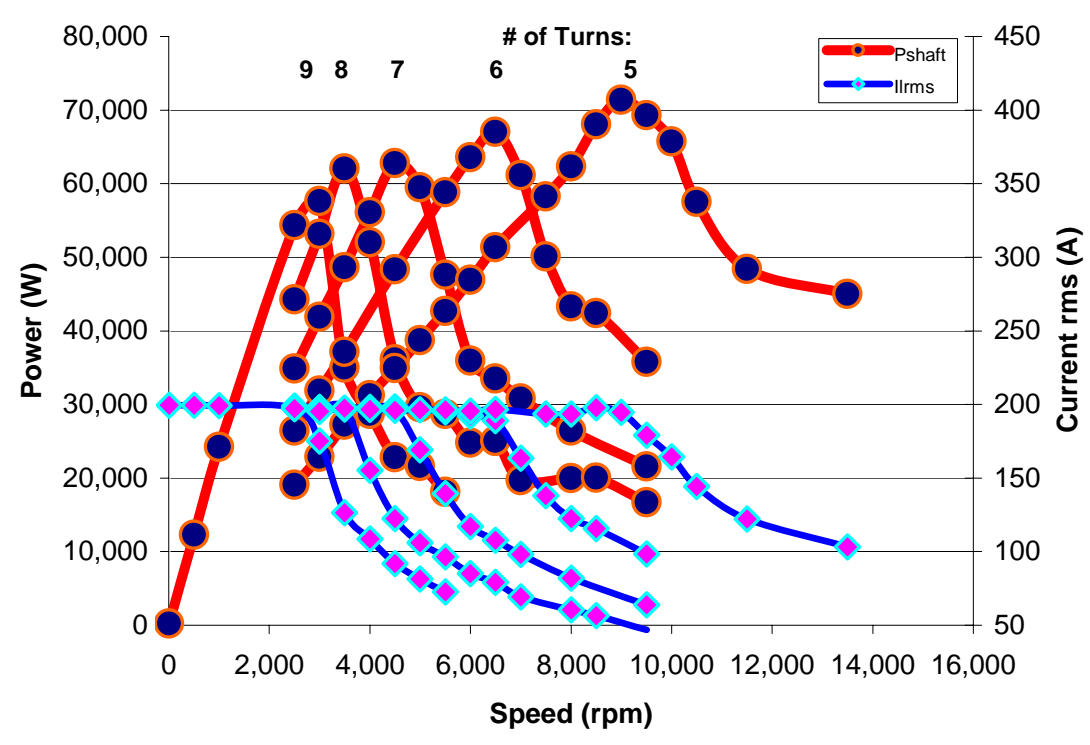

(a) Performance curve dependency on number of turns.

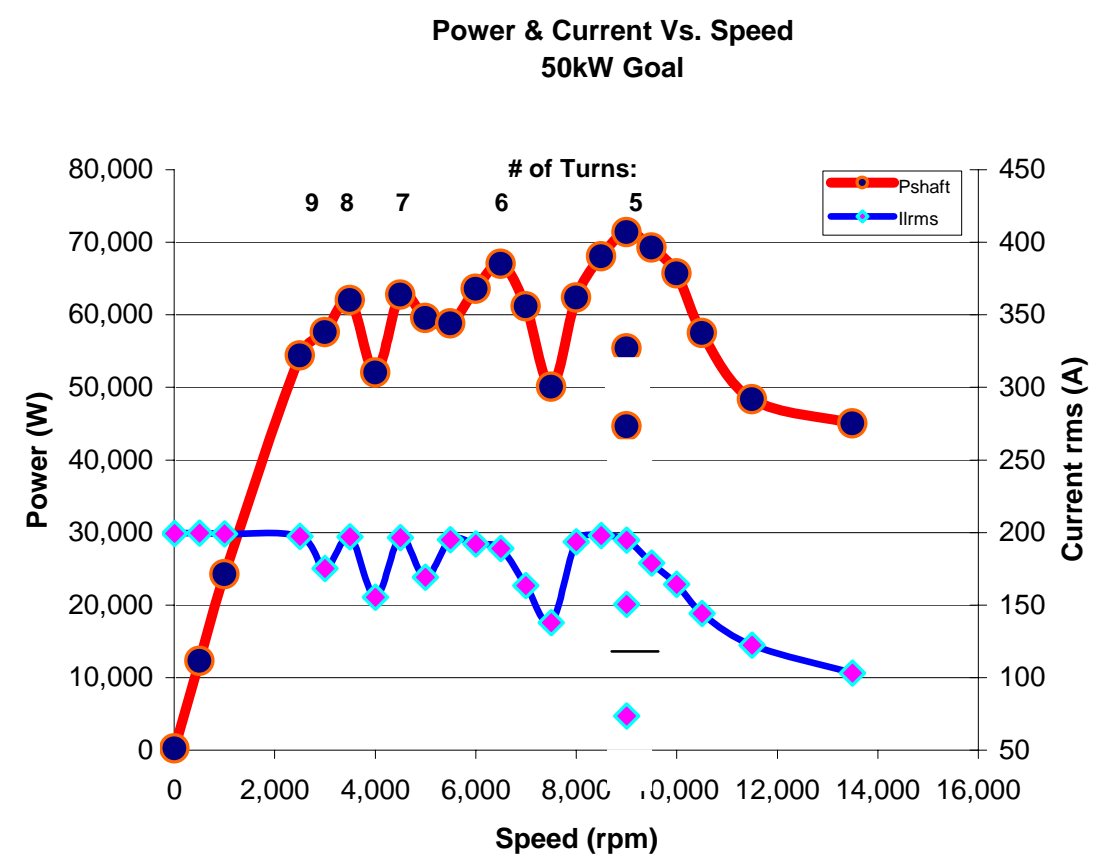

(b) Extending CPSR by reducing the number of turns.

Figure 7. Using the number of turns per slot to control CPSR. 
As shown in Figure 7(a), the number of turns per coil that produces maximum power changes inversely with speed. Analytical determination of the number of turns that delivers maximum power as a function of frequency begins with

$$
P=P\left(\alpha, N_{s}, \omega, L_{d}, L_{q}, \phi, R, V\right),
$$

where

$\alpha$ is the advance angle (the angle between the voltage and the current)

$N_{s}$ is the number of turns per coil

$\omega$ is the rotational frequency, radians/s

$L_{d}$ is the d-axis inductance, $\mathrm{H}$

$L_{d l}$ is the d-axis inductance of a single turn, $\mathrm{H}$

$L_{q}$ is the q-axis inductance, $\mathrm{H}$

$L_{q 1}$ is the q-axis inductance of a single turn, $\mathrm{H}$

$\varphi$ is the flux, V-s

$R$ is the resistance, ohms

$V$ is the supply voltage

The equation, $\partial P / \partial \alpha=0$, is solved for the advance angle that delivers maximum power. The result is

$$
\alpha=\arctan \left(\frac{R-L_{q} \omega}{L_{d} \omega}\right) .
$$

The advance angle is a function of $N_{s}$ because $R=N_{s} R_{l}, L_{q}=N_{s}^{2} L_{q 1}$, and $L_{d}=N_{s}^{2} L_{d l}$; consequently, $\alpha$ as a function of $\mathrm{N}_{\mathrm{s}}$ is substituted into Eq. (1) and the equation,

$$
\partial P\left(\alpha\left\{N_{s}\right\}, N_{s}, \omega, L_{d}, L_{q}, \phi, R, V\right) / \partial N_{s}=0
$$

is solved for $\mathrm{N}_{\mathrm{s}}$. As part of its modeling effort, ORNL has numerically obtained the solution for the number of turns that will achieve maximum power delivery.

\section{Air-gap variation}

A seventh approach to weaken the magnetic field is to mechanically increase the air gap. This technique increases the reluctance, which weakens the field by reducing the magnet's flux. The concept demands precise control and requires energy to move the rotor while it is spinning. Axial-gap motors with one stator are better suited for this type of control than radial-gap motors, because it is easier to control the distance between two flat parallel planes than to control the distance between two low-angle cones. For example, a 0.005 -in. gap increase of an axial-gap motor requires the rotor to be moved along the axis of rotation by 0.005 in., while a radial-gap motor with a $1^{\circ}$ cone requires the rotor to be moved along the axis of rotation by 0.286 in. For example, a $2^{\circ}$ cone requires a 0.143 -in. axial movement to achieve a gap increase of $0.005 \mathrm{in}$. Oh and Emadi have applied this method at the Illinois Institute of Technology to improve the efficiency and operating range of wheel motors [9].

In summary, seven methods of RTFC to increase the speed range while maintaining the efficiency of a PMSM are discussed. Conventional vector control maintains a torque producing quadrature current, $\mathrm{i}_{\mathrm{q}}$, and a magnet field-weakening d-axis current, $i_{d}$. The component of the dc d-axis current must be maintained during operation at high speed and results in increased stator current resistance losses with the accompanying loss in efficiency. Vector control is well established and allows speed range extension by flux weakening. It does require an expensive absolute encoder and fast control algorithms. In general, 
efficiency considerations make those methods of flux control that require temporary intervention or pulses preferable to those requiring continuous action during operation. The most attractive method of RTFC is the pole number change. This has the potential to enhance vector control because it would perform the same current decomposition to form a d-axis current pulse that would demagnetize magnet(s) as operation at high speed begins and re-magnetize magnet(s) as high speed operation ceases. As the speed of a PM motor increases, the number of turns per coil must be reduced to achieve peak power delivery. Reducing the number of turns per coil is also a method of reducing the back-emf to accomplish RTFC. Its benefits are additional power as well as higher-speed operation. Another attractive but complicated method of RTFC is the coil number change, which would have to be used in conjunction with the pole number change because the ratio of coils to poles must remain constant. A number of papers have discussed changing the coil number for an induction motor, which automatically induces fewer poles when the number of coils is reduced. For a PM motor, the number of magnet poles may only be reduced by demagnetization. Mechanical RTFC, such as variable-gap control, can work for an axial-gap PM motor, but it is much more difficult for a radial-gap motor because of the requirement that the gap be precisely controlled by axial motion of a conical rotor. The usual problem remains of energy expended as the gap is opened and closed to accomplish field weakening.

To date, most of the efforts have focused on changing magnetic-flux-flow paths. We feel that the alternatives, which change the magnet properties or number of turns, should be examined for applicability to FreedomCAR machine targets.

\section{Inductance Control}

High-power density and efficiency resulting from elimination of rotor windings and reduced magnetic-flux losses have made the rare earth PM motor a leading candidate for DOE's Office of FreedomCAR and Vehicle Technologies traction drive motor. These traction drives are generally powered by radial-gap motors, having the magnets on or embedded in a rotating cylinder separated from the inside surface of a slotted cylindrical stator by an annular gap. The two main types of radial-gap PM rotors are those with magnets mounted on the surface of a supporting back iron, called SPM motors, and those with magnets mounted in slots in the rotor, called IPM motors.

Most early PM motor research was on the SPM motor, which was thought to have an inherently low stator inductance. A low stator inductance can lead to currents dangerously exceeding rated current as the back-emf across the inductance increases with speed; consequently, part of the attempted solution has been to increase the stator inductance to reduce the rate of current rise. Although analysis suggested that there should be no problem designing sufficiently high stator inductance into SPMs, attempts to do so were often not successful; and a motor design was sought that would have a higher intrinsic inductance.

Commercial research at Toyota has focused on IPM motors because they possess a high saliency ratio, which helps them operate over a high CPSR; but they are more difficult to fabricate. ORNL's position has been to continue research on brushless direct current motors because of ease of fabrication and increased power output.

Recently there has been a revival of interest in a fractional-slot PMSMs [12] made with concentrated windings because they possess three important features. First, they can increase the motor's inductance sufficiently to reduce the characteristic current to the value of the rated current, which will enable them to operate at high CPSR. This feature also limits short-circuit fault currents. Second, their segmented structure simplifies assembly problems and is expected to reduce assembly costs. Third, the back-emf waveform is nearly sinusoidal with low cogging.

In the 1980s, Sneyers, Novotny, and Lipo [25] first investigated the viability of IPM motors as traction devices and were followed in 1987 by Jahns [10]. Then Schiferl and Lipo [26] in 1990 developed the criterion for optimal field-weakening, which is expressed by the equation, $I_{c h}=\frac{\psi_{m}}{L_{d}}$, where $I_{c h}$ is the characteristic current, $\psi_{\mathrm{m}}$ is the magnet's flux linkages, and $L_{d}$ is the inductance. The importance of $I_{c h}$ is summarized by the statement, "If a lossless motor is designed such that its rated current is equal to the 
characteristic current, it will have an infinite CPSR." The seminal paper was that of Soong and Miller [27]. In this paper they normalized the parameters of the motor and introduced the concept of the IPM parameter plane. For the SPM motor, they chose as a design parameter the normalized flux linkage, $\psi_{\mathrm{mn}}$. For optimal performance defined to allow infinite CPSR, this value must be $\psi_{\mathrm{mn}}=1 / 2$. Furthermore, they state that present-day (1980s) SPM motors have values of $\psi_{\mathrm{m}}$ between 0.83 and 0.96. Adnanes and Undeland [28] perform a similar normalization but choose $X_{s}$, the normalized d-axis reactance, as their parameter. For the SPM motor, the optimal value is again $X_{s}=1 / 2$. They further state that $X_{s}$ is typically in the range of $0.3-0.35$. Both papers concentrate on IPM motors and present-day research efforts have followed this trend. While neither paper states that an optimum SPM value cannot be obtained, the general thinking has been that, (1) since the magnet is the only source of air-gap flux density, $\psi_{\mathrm{m}}$ must be large; and (2) since the magnet acts like an additional air gap, the d-axis inductance must be low.

However, the SPM motor is much easier to manufacture than the IPM motor, and research efforts at ORNL have continued with the emphasis on techniques for increasing $L_{d}$ of SPMs.

To examine this design in depth, ORNL entered into a collaborative agreement with UWM to build and test a 6-kW laboratory demonstration unit. Design, fabrication, and testing of the unit to $4000 \mathrm{rpm}$ were completed during FY 2005. The motor will be sent to ORNL to explore ways to control its inverter to achieve higher efficiency during FY 2006.

To review coil parameters, recall that each coil, which has two sides and two ends, is rectangular, with opposite sides referred to as side 1 and side 2 lying in two slots whose angular separation is the coil pitch, $\tau_{\mathrm{p}}$. This angular separation may be defined in mechanical radians, $\omega_{\mathrm{m}}$, or more usefully in electrical radians, $\omega_{\mathrm{e}}$, where $\omega_{\mathrm{e}}=\omega_{\mathrm{m}} \mathrm{p}$ and $\mathrm{p}$ is the number of pole pairs. The angular separation of the two sides determines if the winding is integer-slot or fractional-slot. Angular separation of opposite sides of the coil is referenced to the angular width of one magnet pole so that the word "fractional" in the term "fractionalslot" means the fraction of a magnet covered by the coil.

An important parameter is the number of slots per pole per phase, $N_{s p p}$. If there are 24 slots, 4 poles and 3 phases $\mathrm{N}_{\mathrm{spp}}=2$ and, since this parameter is an integer, we refer to the winding as an integral-slot winding. Typically, these motors have double-layer windings, meaning that each slot will contain coil sides from two different coils; consequently, there are as many coils as slots. This motor could be wound as a single-layer winding with only 12 coils each wound in slots $180^{\circ}$ apart, but there are advantages to the double layer.

PMSMs have sinusoidal back-emfs and thus must have sinusoidal distributed windings. The two choices are (1) full pitch, or (2) fractional pitch, often termed "short chorded" because the chord connecting coil side 1 and coil side 2 is shorter than one required to cross a complete magnet pole. A fractional-pitch winding is determined by the ratio of the coil span to the magnet span. If this ratio is unity, the winding is full pitch; and if the ratio is less than unity, the winding is fractional pitch (short chorded). If this ratio is less than unity, the back-emf will be decreased.

It is well known that a fractional-slot winding has the advantage of behaving as a winding with many slots per pole per phase [29], thus reducing the distribution factors of the harmonics. Liwschitz [30] has developed the distribution and pitch factors of the harmonics of fractional-slot windings. However, these windings were developed for standard ac machines.

With the emergence of the PMSM with IPMs, the sinusoidal-distributed winding became the winding of choice. However, it was recognized that there were disadvantages [11] that increased the costs in the manufacturing process. The stator became a prime target for cost reduction efforts and concentrated coils [11], where each coil is wound around one tooth, became a viable candidate by allowing segmented stator poles and a high slot fill factor (70\% compared with 50\%). Figure 8 shows the different winding configurations. 


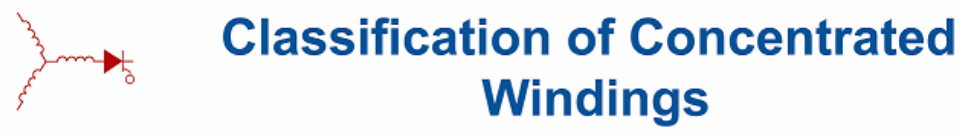

Three Major Categories:

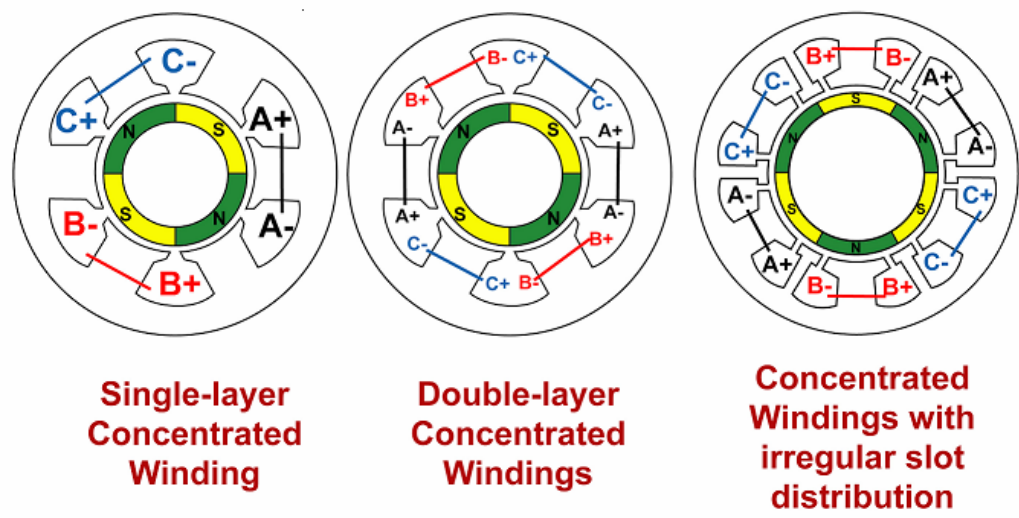

Figure 8. Concentrated winding configurations.

During this time frame, the electromagnetic design and performance characteristics of PMSMs with surface-mounted magnets and concentrated windings were presented in two papers [31, 32]. Two important observations are evident:

(1) There are combinations of slots and poles that allow a fractional-slot concentrated winding to have a high-winding factor and a balanced three-phase output (Table 1 of [31]).

(2) A characteristic of concentrated windings [32] is that they generate both odd and even mmf waves, which leads to increased leakage inductance. This would also increase the d-axis inductance, $L_{d}$, and hence the CPSR of the motor.

The major conclusion of this paper was that SPM machines could be designed to achieve optimal flux-weakening conditions by introducing concentrated fractional-slot stator windings. By optimal conditions, it is meant that the d-axis inductance, $L_{d}$, for infinite CPSR can be achieved.

As of this time frame, there were no previous publications that describe specific design techniques for applying such windings to achieve optimal flux-weakening conditions in SPM machines. This need was then met by El-Refaie and Jahns [12] of the University of Wisconsin when they developed a design technique and applied it to the design of a 36-slot, 42-pole PMSM with fractional-slot concentrated winding and surface-mounted magnets. The results showed promise as a candidate for the FCVT program, and ORNL entered into collaboration with the University of Wisconsin to build and test a 6-kW, 36-slot/30-pole PMSM with SPMs and concentrated fractional-slot windings. ORNL modeled the motor using SPEED software in order to verify its performance against the UWM test data.

The 6-kW, 36-slot/30-pole concentrated winding fraction-slot motor has now completed its verification testing cycles, and both the results and the analytical techniques used in the design are published [12]. The basic repeating unit of the SPM machine is shown in Figure 9.

The design data and calculated parameters are summarized in Tables 1-5. The analytical and experiment results of the verification tests are shown in Table 6. 


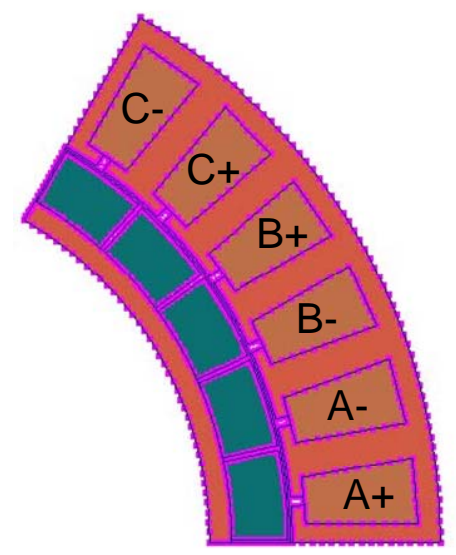

Figure 9. Basic repeating unit of the 6-kW SPM motor with fractional-slot concentrated windings [12].

Table 1. Stator dimensions and winding data for the 36-slot/ 30-pole SPM machine [12]

\begin{tabular}{|c|c|c|c|}
\hline Number of slots & 36 & Number of poles & 30 \\
\hline Number of phases & 3 & Slots/pole/phase & $2 / 5$ \\
\hline Series turns & 108 & Number of turns/coil & 18 \\
\hline Number of coils & 6 & $\begin{array}{c}\text { Number of parallel } \\
\text { paths }\end{array}$ & 1 \\
\hline Outer diameter & $280[\mathrm{~mm}]$ & Active length & $60[\mathrm{~mm}]$ \\
\hline Total length & $72[\mathrm{~mm}]$ & Slot fill factor & $35 \%$ \\
\hline Slot opening width & $2[\mathrm{~mm}]$ & Slot bottom width & $10[\mathrm{~mm}]$ \\
\hline Slot top width & $14.6[\mathrm{~mm}]$ & Slot opening height & $3[\mathrm{~mm}]$ \\
\hline Slot height & $25.4[\mathrm{~mm}]$ & Back iron depth & $9[\mathrm{~mm}]$ \\
\hline Tooth width & $11.4[\mathrm{~mm}]$ & Phase resistance & $63[\mathrm{~m} \Omega]$ \\
\hline
\end{tabular}

Table 2. Rotor dimensions for the 36-slot/30-pole SPM machine [12]

\begin{tabular}{|c|c|c|c|}
\hline $\begin{array}{c}\text { Rotor outer } \\
\text { radius }\end{array}$ & $\begin{array}{c}88.6 \\
{[\mathrm{~mm}]}\end{array}$ & $\begin{array}{c}\text { Magnet outer } \\
\text { radius }\end{array}$ & $101.6[\mathrm{~mm}]$ \\
\hline Inner radius & $\begin{array}{c}63.6 \\
{[\mathrm{~mm}]}\end{array}$ & $\begin{array}{c}\text { Air gap } \\
\text { thickness }\end{array}$ & $1[\mathrm{~mm}]$ \\
\hline $\begin{array}{c}\text { Magnet } \\
\text { depth }\end{array}$ & $13[\mathrm{~mm}]$ & Magnet span & $11.4^{\mathrm{o}}[\mathrm{mech}]$ \\
\hline
\end{tabular}

Table 3. Calculated inductances for the 36-slot/30-pole SPM machine [12]

\begin{tabular}{|c|c|l|c|}
\hline $\begin{array}{c}\text { Self inductance } \\
\text { (including harmonic } \\
\text { leakage) }\end{array}$ & $225[\mu \mathrm{H}]$ & $\begin{array}{l}\text { Mutual inductance } \\
\text { (including } \\
\text { harmonic leakage) }\end{array}$ & $\sim 0$ \\
\hline $\begin{array}{c}\text { Self slot leakage } \\
\text { inductance }\end{array}$ & $805[\mu \mathrm{H}]$ & $\begin{array}{l}\text { Mutual slot } \\
\text { leakage inductance }\end{array}$ & $\sim 0$ \\
\hline $\begin{array}{c}\text { Total self } \\
\text { inductance }\end{array}$ & $1.03[\mathrm{mH}]$ & $\begin{array}{l}\text { Net mutual } \\
\text { inductance }\end{array}$ & $\sim 0$ \\
\hline
\end{tabular}


Table 4. Calculated current and magnet parameters for the 36-slot/30-pole SPM machine [12]

\begin{tabular}{|l|c|l|c|}
\hline $\begin{array}{l}\text { Magnet remanent flux } \\
\text { density, } \mathrm{B}_{\mathrm{r}}\end{array}$ & 0.55 [Tesla] @ $100^{\circ} \mathrm{C}$ & $\begin{array}{l}\text { Magnet relative permeability, } \\
\mu_{\mathrm{r}}\end{array}$ & 1.22 \\
\hline RMS PM flux linkage, $\Psi_{\mathrm{m}}$ & $34.8\left[\mathrm{mWb} \mathrm{rms}_{\mathrm{rm}}\right]$ & $\begin{array}{l}\text { RMS characteristic current, } \\
\mathrm{I}_{\mathrm{ch}}=\Psi_{\mathrm{m}} / \mathrm{L}_{\mathrm{d}}\end{array}$ & $33.8\left[\mathrm{~A}_{\mathrm{rms}}\right]$ \\
\hline RMS rated current, $\mathrm{I}_{\mathrm{R}}$ & $43[\mathrm{Arms}]$ & $\begin{array}{l}\text { Flux=weakening index FWI } \\
=\mathrm{I}_{\mathrm{ch}} / \mathrm{I}_{\mathrm{R}}\end{array}$ & 0.79 \\
\hline Copper current density & $7\left[\mathrm{~A}_{\mathrm{rms}} / \mathrm{mm}^{2}\right]$ & Air-gap shear stress & $3.1[\mathrm{psi}]=21[\mathrm{kPa}]$ \\
\hline
\end{tabular}

Table 5. Breakdown of material mass for the 36-slot/30-pole SPM machine [12]

\begin{tabular}{|l|c|l|c|}
\hline Copper mass & $2.8[\mathrm{~kg}]$ & Iron mass & $12.6[\mathrm{~kg}]$ \\
\hline Magnet mass & $3.6[\mathrm{~kg}]$ & Total mass & $19[\mathrm{~kg}]$ \\
\hline
\end{tabular}

Table 6. Verification test results [12]

\begin{tabular}{|c|c|c|c|c|c|c|c|c|c|c|}
\hline & & \multicolumn{8}{|c|}{$100 \%$ load } & \multirow[t]{2}{*}{$\mathrm{P}_{\text {out }} / \mathrm{P}_{\mathrm{it}}$} \\
\hline $\mathrm{rpm}$ & mech rad/s & $\overline{I_{q}}$ & $\mathrm{I}_{\mathrm{d}}$ & $\mathrm{I}$ & $V_{\text {phase }}$ & $\mathrm{P}_{\mathrm{in}}=3 \mathrm{I} \mathrm{V}_{\text {phase }}$ & $\mathrm{T}_{\text {out }}$ & $\mathrm{P}_{\text {out }}$ & Eff & \\
\hline & & Amps & Amps & Amps & Volts & Volt-amps & $\mathrm{N}-\mathrm{m}$ & Watts & $\%$ & \\
\hline 800 & 83.78 & 43.0 & 0.0 & 43 & 85.0 & 10965 & 60 & 4901 & 86.0 & 44.7 \\
\hline 2000 & 209.44 & 21.6 & -26.7 & 34.3 & 87.7 & 9035.675 & 30.5 & 6392 & 91.0 & 70.74 \\
\hline 3000 & 314.16 & 14.4 & -26.5 & 30.2 & 87.7 & 7935.028 & 20.4 & 6415 & 92.0 & 80.84 \\
\hline 4000 & 418.88 & 10.8 & -26.4 & 28.5 & 87.7 & 7504.579 & 15.3 & 6404 & 91.9 & 85.33 \\
\hline \multicolumn{11}{|c|}{ Experimental } \\
\hline 800 & 83.78 & 43.0 & 0.0 & 43 & 81.0 & 10449 & 64 & 5400 & 84.0 & 51.68 \\
\hline 2000 & 209.44 & 24.8 & -26.9 & 36.6 & 89.0 & 9768.88 & 29 & 6075 & 90.3 & 62.19 \\
\hline 3000 & 314.16 & 18.4 & -14.6 & 23.5 & 91.0 & 6412.421 & 19.4 & 6099 & 90.0 & 95.11 \\
\hline 4000 & 418.88 & 16.6 & -14.9 & 22.3 & 91.0 & 6089.614 & 14.7 & 6146 & 91.7 & 100.9 \\
\hline
\end{tabular}

It is noteworthy that bonded neodymium-iron-boron magnets were used to minimize eddy-current losses in the magnets. Because of its relatively low $\mathrm{B}_{\mathrm{r}}(0.55 \mathrm{~T})$ and relative permeability $\mu_{\mathrm{r}}(1.22)$, its length of $13 \mathrm{~mm}$ is large compared with that of an induction motor (IM). Also unlike IMs, the slotleakage inductance was almost a factor of four higher than the self inductance.

The important result is shown in Table 4 which shows the flux-weakening index, $\mathrm{FWI}=\mathrm{I}_{\mathrm{ch}} / \mathrm{I}_{\mathrm{r}}=0.79$. This means the d-axis inductance, $L_{d}$, is higher than the optimal value so that the rated current is smaller than the characteristic current; however, as seen in Table 7 and in [13], the motor met its design goal of CPSR $=6$.

We now compare the results with the results of the ORNL calculations, which employ the SPEED software. The motor as represented in the SPEED program is shown in Figure 10. Each of the 18 coils is wrapped around a separate tooth, and each slot has only one coil side inserted. Figure 11 is the winding diagram for one phase. 


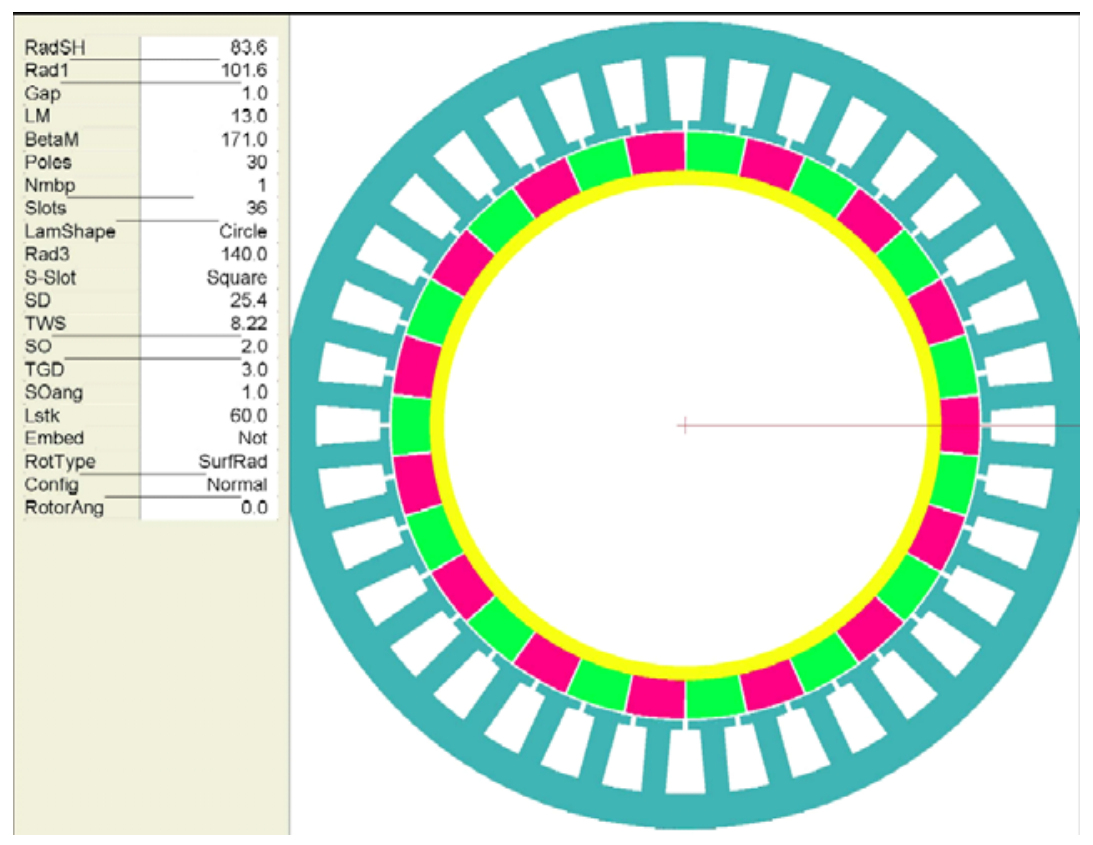

Figure 10. Configuration of the ORNL model of a $6 \mathrm{~kW}$ fractionalslot PMSM motor made with concentrated windings.

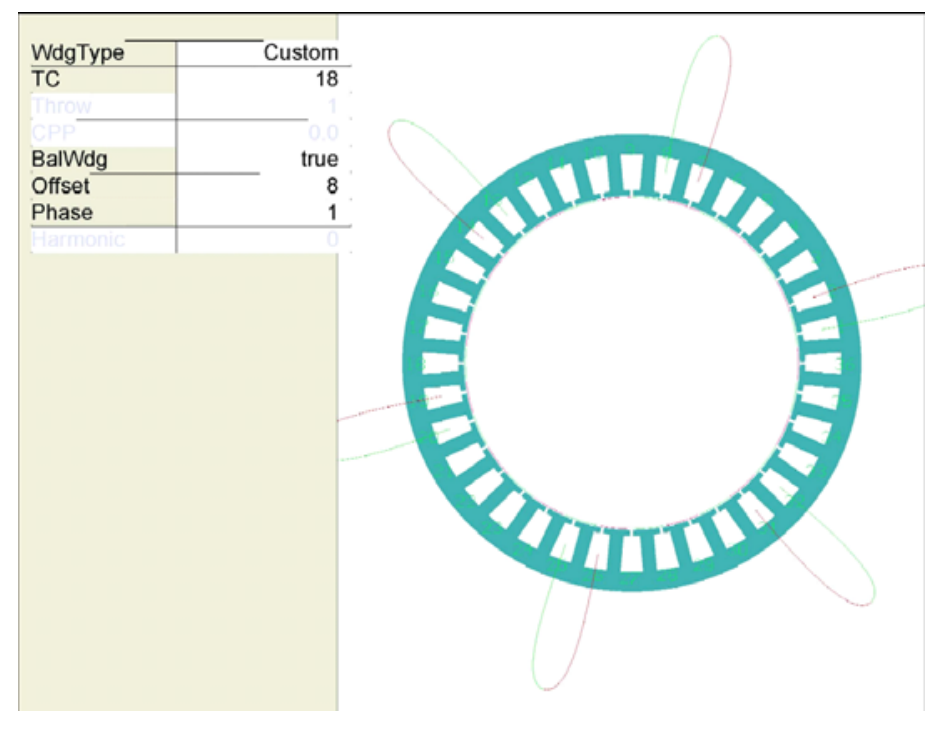

Figure 11. One phase of the coil winding configuration for the ORNL model of a $6 \mathrm{~kW}$ fractional-slot PMSM motor with concentrated windings. 
Calculations by UWM and ORNL for the 6-kW fractional-slot motor with concentrated windings are compared in Table 7.

Table 7. Comparison of $6 \mathrm{~kW}$ fractional-slot motor analyses

\begin{tabular}{|c|c|c|}
\hline Parameters per phase & Wisconsin & ORNL (SPEED software) \\
\hline Gap inductance & $0.225 \mathrm{mH}$ & $0.293 \mathrm{mH}$ \\
\hline Slot inductance & $0.805 \mathrm{mH}$ & $0.767 \mathrm{mH}$ \\
\hline End-turn inductance & - & $0.04 \mathrm{mH}$ \\
\hline Total stator inductance & $1.03 \mathrm{mh}$ & $1.1 \mathrm{mh}$ \\
\hline Resistance & $0.063 \mathrm{~m} \Omega$ & $0.047 \mathrm{~m} \Omega$ \\
\hline Back-emf & $41.87 \mathrm{rms}$ volts & $55.75 \mathrm{rms}$ volts \\
\hline
\end{tabular}

The most significant deviation is the $33 \%$ difference in the back-emf. In discussions with UWM, it was determined that SPEED uses a one-dimensional analysis to model the air-gap flux density, whereas UWM uses the technique of Zhu [33], which is based on a two-dimensional solution of LaPlace's equation. Using this technique, the back-emf of Figure 12 was generated.

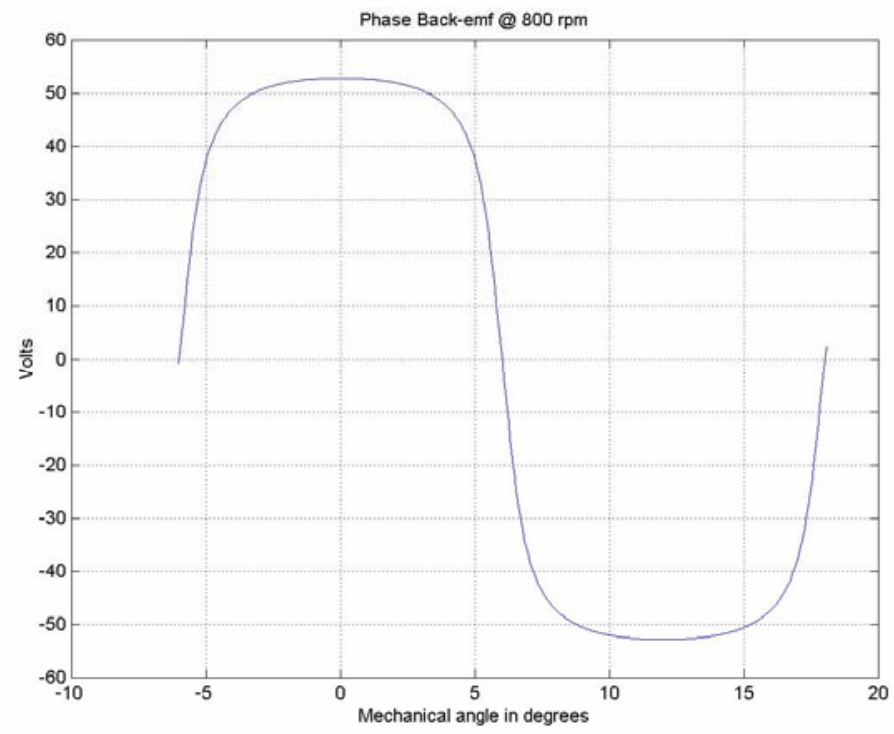

Figure 12. Peak back-emf per phase of $6 \mathrm{~kW}$ motor at $800 \mathrm{rpm}$.

From its harmonic content, shown in Figure 13, the fundamental rms back-emf per phase at $800 \mathrm{rpm}$ becomes, $E=64 / \sqrt{2}=45 \mathrm{~V}_{\text {phase }}^{r m s}$, which agrees well with the UWM value of $41.9 \mathrm{~V}_{\text {rms. }}$.

In general, there is good correlation between the ORNL and UWM analyses and the actual motor response. The 6-kW motor, as analyzed in the SPEED program, can be approximately modeled by the circuit of Figure 14. The winding resistances differ, but they do not dramatically affect the results.

Slotting influences the magnetic field in two ways. First, it reduces the total flux per pole and increases the effective air gap. This effect is accounted for by the Carter Coefficient, $\mathrm{k}_{\mathrm{c}}$. It also affects the distributions of the flux in both the air gap and the magnets. To account for the second effect, the Fourier permeance function, $\lambda(\theta)$, is introduced in Figure 15. The back-emf is then multiplied by this function to obtain the corrected back-emf. 


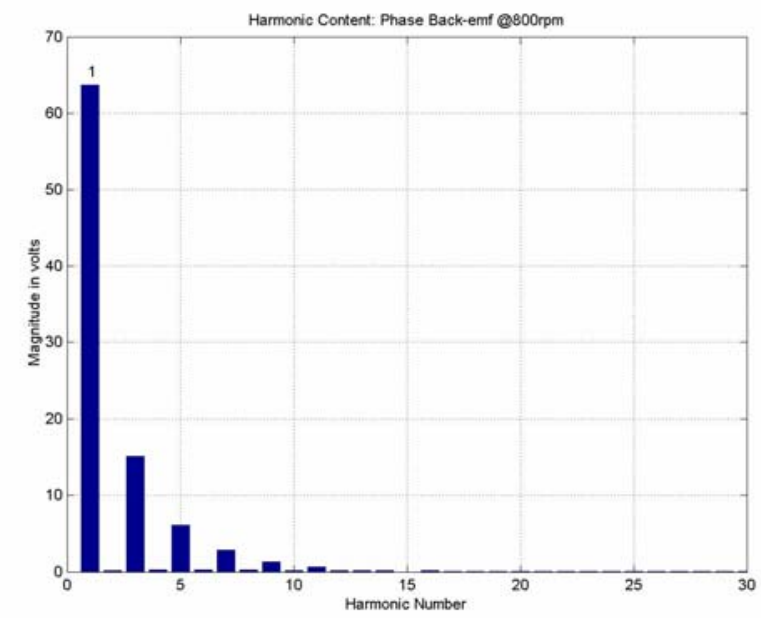

Figure 13. Harmonic content in back-emf of $6 \mathrm{~kW}$ motor at $800 \mathrm{rpm}$.

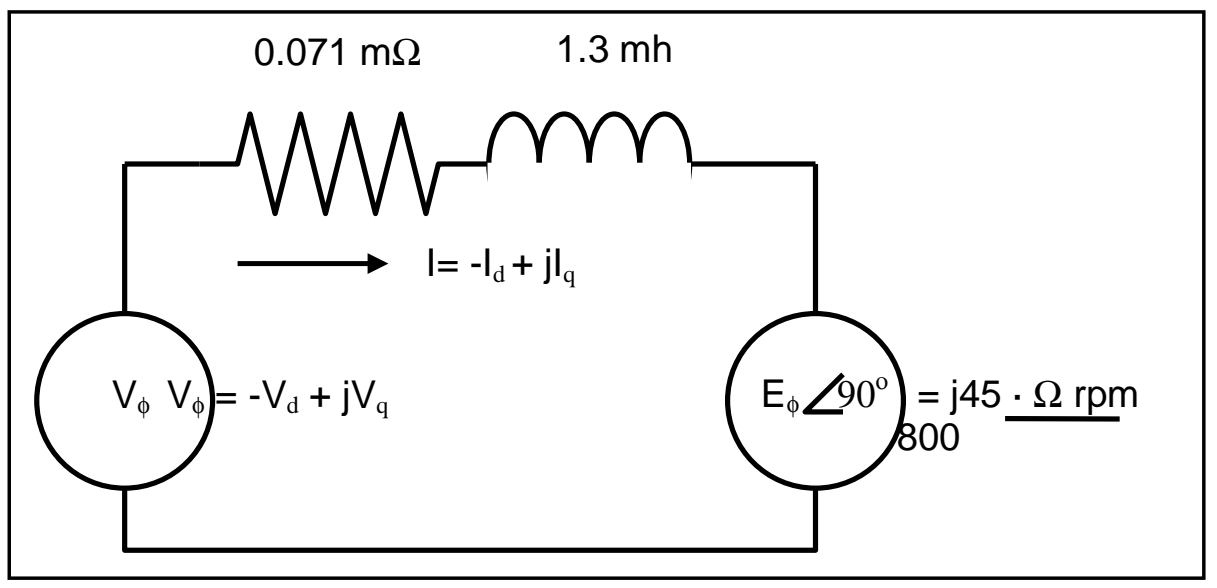

Figure 14. Per-phase equivalent circuit of $6 \mathrm{~kW}$ motor.

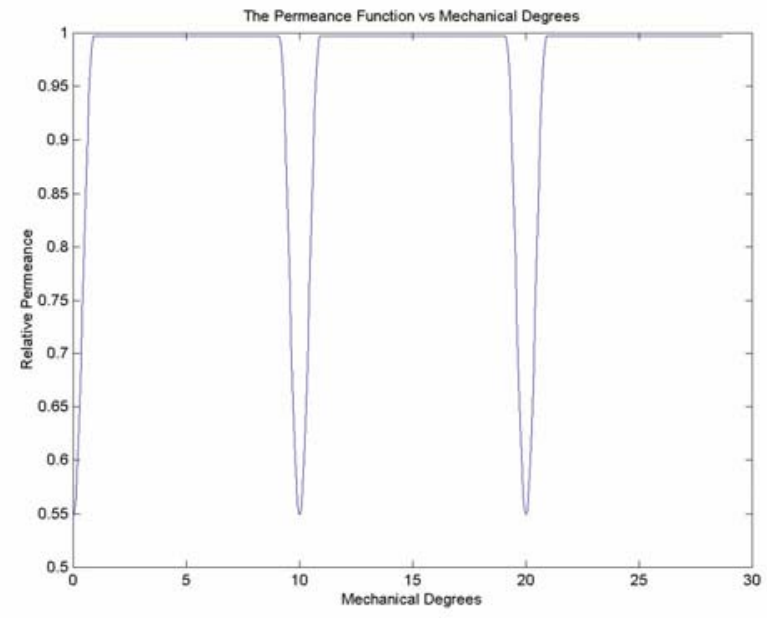

Figure 15. Relative permeance. 


\section{Inverter Control}

It is well known that the ability of the PMSM to operate over a wide CPSR is dependent upon the machine inductance [15, 16, 28, 29, 34]. Early approaches for extending CPSR operation included adding supplementary inductance in series with the motor [15] and the use of anti-parallel thyrsistor pairs in series with the motor phase windings [16]. The increased inductance method is compatible with a VSI controlled by PWM, which is called CPA. The thyristor method has been called DMIC. Neither of these techniques has met with wide acceptance since they both add cost to the drive system and have not been shown to have an attractive cost/benefit ratio. Recently, a method has been developed to use fractionalslot concentrated windings to significantly increase the machine inductance [12]. This latest approach has the potential to make the PMSM compatible with CPA without supplemental external inductance. If the performance of such a drive is acceptable, then the method may make the PMSM an attractive option for traction applications requiring a wide CPSR.

A 30-pole, 6-kW, 6000-maximum-rpm prototype of the fractional slot PMSM design has been developed [35]. This machine has significantly more inductance than is typical of regular PMSMs. The prototype is to be delivered in late 2005 to ORNL for testing and development of a suitable controller. In advance of the test/control development effort, ORNL has used the PMSM models developed over a number of previous studies to evaluate the steady state performance of high-inductance PMSM machines with a view toward control issues. The detailed steady state model developed includes all motor and inverter loss mechanisms and will be useful in assessing the performance of the dynamic controller to be developed in future work.

The main problem found with high-inductance machines driven by CPA is that the motor current at high speed depends solely on machine parameters and is virtually independent of load level and the dc supply voltage. Thus the motor current is virtually the same at no load as at full load, resulting in poor efficiency at less than full-load conditions. DMIC is shown to produce a motor current at high speed that is proportional to load and has the potential to maintain high efficiency at full- and partial-load conditions [14]. However, an important concern with DMIC is found to be the reverse recovery losses of the thyristors at high speed. Because the turn-off of the thyristors in DMIC is naturally soft, it is possible that economical converter-grade components can be used, provided the fundamental switching frequency is about $1 \mathrm{kHz}$ or less. The high pole count (30 poles) of the prototype motor results in a fundamental rate of $1.5 \mathrm{kHz}$ at a top speed of 6,000 rpm and results in substantial recovery losses in the thyristors. The reverse recovery losses can be reduced by using inverter-grade components, which are available but cost more. The cost and additional losses associated with the thyristors used in DMIC may be offset by efficiency enhancements.

Another significant issue regarding high-inductance PMSMs uncovered in this study involves the amount of inductance required to meet CPSR requirements. It is generally believed that there is an "optimal value" for field weakening given by

$$
L_{\infty}=\frac{E_{b}}{\Omega_{b} I_{R}}
$$

where $E_{b}$ is the rms magnitude of the line-to-neutral back-emf at base speed, $\Omega_{b}$ is the base speed in electrical radians per second, and $I_{R}$ is the rms current rating of the motor windings. The prototype machine that is to be delivered to ORNL has about 1.7 times more inductance than was calculated using the original design equations. The inventors of the fractional slot concentrated winding method, and developers of the prototype machine, remarked that they were "too successful" in incorporating inductance into their machine and that steps would be taken to modify the design methodology to reduce the inductance to the optimum value. This study will show a significant advantage of having the higher inductance rather than the "optimal" value. Specifically, it is shown that the higher inductance enables the motor to develop the required power at lower current, thereby reducing motor and inverter losses and 
improving efficiency. While an inductance higher than the value cited above is warranted, it still does not make the motor current proportional to load. Consequently, the problem of low efficiency at high speed and less than full load is not resolved.

A final point uncovered in this study concerns the dc supply voltage that provides the underlying source for the traction drive. A common definition of "base speed" is the speed at which the voltage applied to the motor armature is equal to the magnitude of the back-emf. The results in this study indicate that the dc supply voltage should be adequate to drive rated current into the motor winding at the specified base speed. At a minimum, this requires sufficient voltage to overcome not only the back-emf but also the voltage drop across the internal impedance of the machine. For a high-inductance PMSM, the internal impedance at base speed can be considerable, and substantial additional voltage is required to overcome the internal voltage drop. It is further shown that even more voltage than the minimum required for injecting rated current at base speed can be beneficial. In particular, this allows the required power to be developed at lower current, thereby reducing losses in the motor and in the inverter components. Further, it is shown that the current is minimized at a unique speed that varies with voltage.

Consequently, there may be room for optimization. For example, if the drive spends a substantial amount of its operating life in the vicinity of one-half of maximum speed, then it can be desirable to choose a dc supply voltage that causes the motor current to achieve its minimum value at half full speed.

The findings of this study are demonstrated on two example motors whose parameters are given in Table 8. Motor 2 is patterned after the high-inductance prototype that is to be delivered to ORNL. Motor 1 has the characteristic inductance value as indicated by the formula above. The parameters of the two cases are identical except for the winding inductance. The parameters in Table 8 were taken from ref. [13].

The detailed technical assessment of Motor 1 and Motor 2 includes the evaluation of losses, not only in the motor but also in the inverter. The motor losses are in the copper resistance and the speed-sensitive rotational losses. The transistors in the inverter are typically controlled by sinusoidal PWM. A detailed PSPICE simulator is available to analyze the performance of the PMSM controlled by PWM when operating at constant speed. A simplified per-phase fundamental frequency model validated and used for this study is shown in Figure 16. It is a phasor model of the motor drive at a selectable but constant speed.

In the per phase model of Figure 16, the phasor $\overline{\mathrm{V}}$ represents the fundamental frequency line-toneutral voltage applied to the motor by the inverter. $\mathrm{V}$ is the rms magnitude and $\delta$ is the inverter lead angle. Phasor $\overline{\mathrm{E}}$ represents the phase-to-neutral motor back-emf and is chosen as the reference phasor, such that the angle of $\overline{\mathrm{E}}$ is zero.

Neglecting the armature resistance, the phasor current of the motor is

$$
\begin{aligned}
\bar{I} & =\frac{V_{\max }}{n X_{b}} \sin \delta+j\left[\frac{E_{b}}{X_{b}}-\frac{V_{\max }}{n X_{b}} \cos \delta\right], \\
& =I_{r}+j I_{x}
\end{aligned}
$$

where $I_{r}$ is the torque producing component of current in phase with the back-emf. This component is like the q-axis current in the d-q model. $I_{x}$ is the component of current that is orthogonal to the back-emf and results in no net torque production. This is the field weakening component of current and is like the daxis current in the $\mathrm{d}-\mathrm{q}$ model. The total motor current has an rms magnitude of 


$$
\begin{aligned}
I & =\sqrt{I_{r}^{2}+I_{x}^{2}} \\
& =\frac{\sqrt{V_{\max }^{2}-n 2 V_{\max } E_{b} \cos \delta+n^{2} E_{b}^{2}}}{n X_{b}} .
\end{aligned}
$$

\begin{tabular}{|c|c|c|}
\hline Parameter & Motor 1 & Motor 2 \\
\hline Number of poles & 30 & 30 \\
\hline Base speed & $900 \mathrm{rpm}$ & $900 \mathrm{rpm}$ \\
\hline Top speed & $6000 \mathrm{rpm}$ & $6000 \mathrm{rpm}$ \\
\hline CPSR requirement & $6.667: 1$ & $6.667: 1$ \\
\hline $\begin{array}{l}\text { Back-emf magnitude at base speed, } \mathrm{E}_{\mathrm{b}} \\
\text { (root-mean-square (rms) volts per phase) }\end{array}$ & $46.5 @ 900 \mathrm{rpm}$ & 46.5 @ 900 rpm \\
\hline $\begin{array}{l}\text { Voltage constant, } \mathrm{K}_{\mathrm{v}} \\
\text { (rms volts per elec. } \mathrm{rad} / \mathrm{sec})\end{array}$ & 0.03289 & 0.03289 \\
\hline Rated power & $6 \mathrm{~kW}$ & $6 \mathrm{~kW}$ \\
\hline Rated torque & $63.66 \mathrm{Nm}$ & $63.66 \mathrm{Nm}$ \\
\hline Rated rms current & $43.0 \mathrm{~A}$ & $43.0 \mathrm{~A}$ \\
\hline Resistance per phase & $71 \mathrm{~m} \Omega$ & $71 \mathrm{~m} \Omega$ \\
\hline Inductance per phase & $765 \mu \mathrm{H}$ & $1300 \mu \mathrm{H}$ \\
\hline \multicolumn{3}{|l|}{ Rotational losses $P_{r o t}(n) @ \mathrm{n}=\mathrm{rpm} / 900$} \\
\hline $1000 \mathrm{rpm}(\mathrm{n}=1.11)$ & $8.3 \mathrm{~W}$ & $8.3 \mathrm{~W}$ \\
\hline $2000 \mathrm{rpm}(\mathrm{n}=2.22)$ & $33.3 \mathrm{~W}$ & $33.3 \mathrm{~W}$ \\
\hline $3000 \mathrm{rpm}(\mathrm{n}=3.33)$ & $75 \mathrm{~W}$ & $75 \mathrm{~W}$ \\
\hline $4000 \mathrm{rpm}(\mathrm{n}=4.44)$ & $133.3 \mathrm{~W}$ & $133.3 \mathrm{~W}$ \\
\hline $5000 \mathrm{rpm}(\mathrm{n}=5.56)$ & $208.3 \mathrm{~W}$ & $208.3 \mathrm{~W}$ \\
\hline $6000 \mathrm{rpm}(\mathrm{n}=6.67)$ & $300 \mathrm{~W}$ & $300 \mathrm{~W}$ \\
\hline
\end{tabular}

Table 8. Parameters of Motor 1 and Motor 2

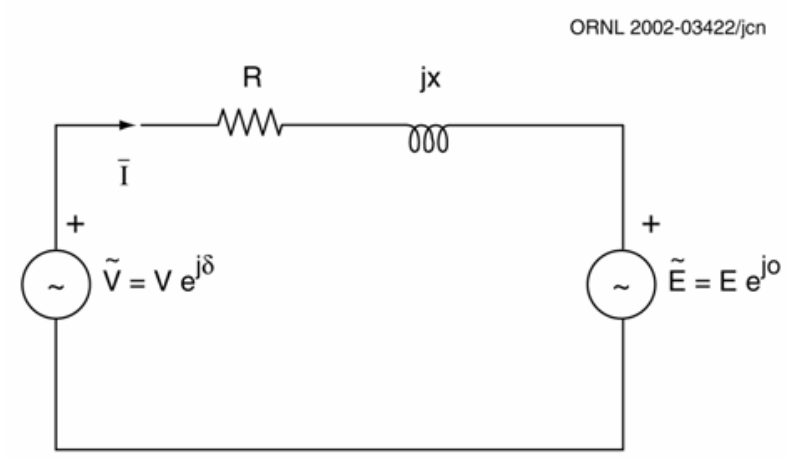

Figure 16. Fundamental frequency model of per-phase of a PMSM. 
The total power injected into the motor by the inverter is

$$
P_{i n}=3 \operatorname{Re}\left(\bar{V} \bar{I}^{*}\right)=\frac{3 V_{\max } E_{b}}{X_{b}} \sin \delta,
$$

while the total power converted by the motor is

$$
\begin{aligned}
P_{m} & =3 \operatorname{Re}\left(\bar{E} \bar{I}^{*}\right)=\frac{3 V_{\max } E_{b}}{X_{b}} \sin \delta \\
& =P_{\max } \sin \delta
\end{aligned}
$$

where

$$
P_{\max }=3 \frac{V_{\max } E_{b}}{X_{b}}
$$

is the maximum power that can possibly be converted, which corresponds to the lead angle being $90^{\circ}$. Since we have neglected the winding resistance, $P_{i n}$ equals $P_{m}$ and the common value is

$$
\begin{aligned}
P_{m} & =P_{i n}=\frac{3 V_{\text {max }} E_{b}}{X_{b}} \sin \delta \\
& =P_{\text {max }} \sin \delta
\end{aligned} .
$$

This expression shows that it is easy to control the motor to deliver rated power above base speed. All that is necessary is that the inverter lead angle, $\delta$, be held fixed at that value that causes $P_{m}$ to be equal to the rated value, $P_{R}$, so that

$$
\begin{aligned}
\delta & =\sin ^{-1}\left(\frac{X_{b} P_{R}}{3 V_{\max } E_{b}}\right) \\
& =\cos ^{-1}\left(\frac{E_{b}}{V_{\max }}\right)
\end{aligned}
$$

Observe that, as the speed becomes unbounded, the motor current magnitude approaches a limiting value given by

$$
\begin{aligned}
& \lim _{n \rightarrow \infty} I_{r}=\lim _{n \rightarrow \infty} \frac{V_{\max }}{n X_{b}} \sin \delta=0 \\
& \lim _{n \rightarrow \infty} I_{x}=\lim _{n \rightarrow \infty}\left(\frac{E_{b}}{X_{b}}-\frac{V_{\max }}{n X_{b}} \cos \delta\right)=\frac{E_{b}}{X_{b}}=\frac{E_{b}}{\Omega_{b} L} \\
& \lim _{n \rightarrow \infty} I=\lim _{n \rightarrow \infty} \frac{\sqrt{V_{\max }^{2}+n(n-2) E_{b}^{2}}}{n X_{b}}=\frac{E_{b}}{X_{b}}=\frac{E_{b}}{\Omega_{b} L}=I_{C H}
\end{aligned}
$$

The limiting rms current magnitude is called the "characteristic current" [12] denoted as $I_{C H}$. 
The following information is for DMIC operation of a PMSM. A recent paper [14] used a fundamental frequency model to analyze the performance of the sinusoidal back-emf PMSM driven in constant power mode by DMIC. The inverter includes a common three-phase voltage source inverter supplemented with an alternating current (ac) voltage controller between the inverter output and the motor. The ac voltage controller consists of three pairs of anti-parallel SCRs as shown in Figure 17. Each SCR pair is a full ac switch. In steady state, the fundamental frequency components of the voltage across and current through the switch are $90^{\circ}$ out of phase, reflecting the lossless behavior of the switch and giving rise to an "equivalent reactance" interpretation of the SCRs. On a per-phase basis, a fundamental frequency phasor model has the form shown in Figure 18 with winding resistance and rotational losses neglected. In the figure, the parameter $X_{\text {thy }}$ is the equivalent reactance of an SCR pair.

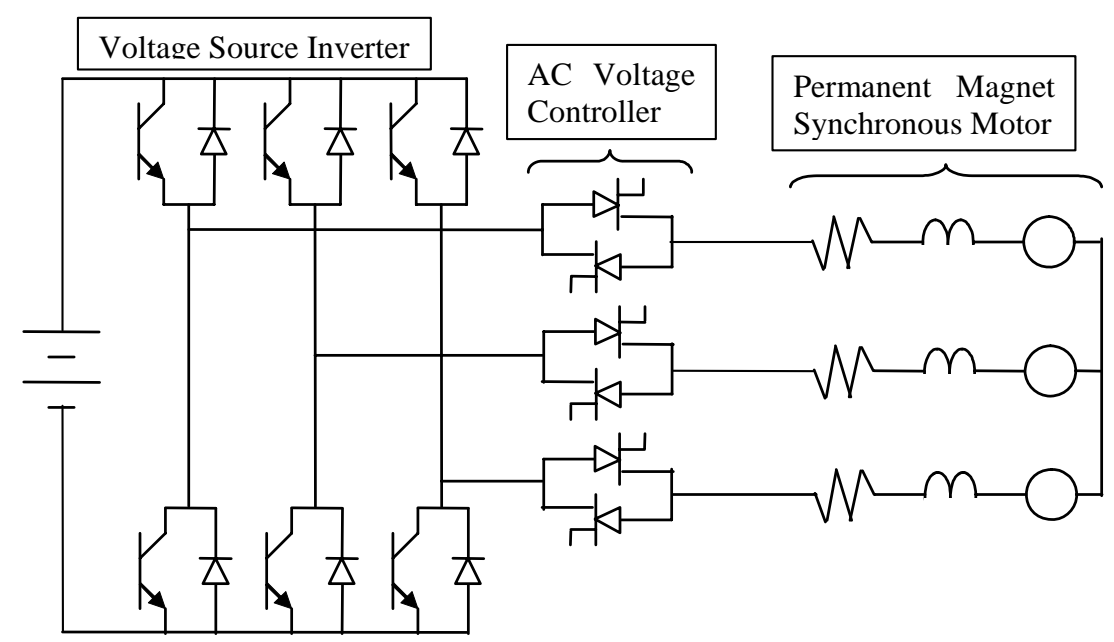

Figure 17. DMIC inverter topology.

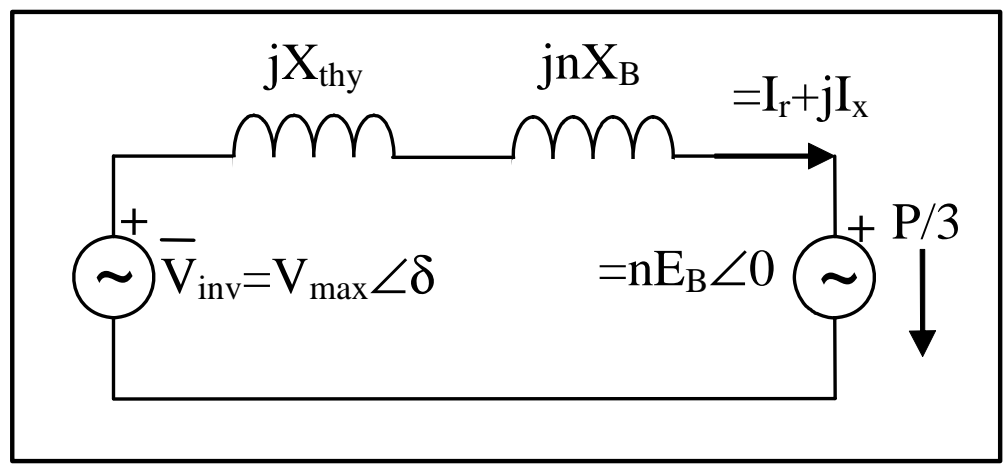

Figure 18. Per-phase fundamental frequency phasor model for constant power mode.

As noted in [14], the equivalent reactance of the ac switch is not constant but varies with the firing angle of the SCRs. The firing angle of the SCRs also controls the developed power of the motor. Since the equivalent reactance of the switch varies with the developed power, one cannot infer that the equivalent reactance interpretation can be extended to a fixed equivalent inductance that is in series with the motor winding. The total reactance presented to the inverter is the sum of the thyristor reactance and 
the motor reactance, $X_{t h y}+n X_{b}$. Thus no matter how small the machine reactance may happen to be, the thyristor reactance can be adjusted, through firing angle control, to make the motor behave as though it were a high-reactance machine. This is why the DMIC achieves an infinite CPSR even when the motor inductance is less, even substantially less, than the minimum required to be driven by CPA.

The inverter lead angle depends on the dc supply voltage and the developed power and is found to be

$$
\delta=\sin ^{-1}\left(\frac{X_{b} P}{3 V_{\max } E_{b}}\right),
$$

and the resulting rms motor current at speed, $n$, is

$$
I=\frac{\sqrt{V_{\max }^{2}-2 n V_{\max } E_{b} \cos \delta+n^{2} E_{b}^{2}}}{n X_{b}} .
$$

Note that at any finite speed the rms current depends, at least to some degree, on dc supply voltage through the dependence on $V_{\max }$, on the developed power through the dependence on $\delta$, and on motor parameters, $E_{b}$ and $X_{b}$. However, at high speed, the rms current approaches the "characteristic current" given by

$$
\lim _{n \rightarrow \infty} I=\frac{E_{b}}{X_{b}}=\frac{E_{b}}{\Omega_{b} L} .
$$

The characteristic current for DMIC operation depends only on motor parameters.

It may be shown that the phase quadrature component of motor current is

$$
I_{x}=\frac{n E_{b}-V_{\max } \sqrt{1-\left(\frac{X P}{3 n E_{b} V_{\max }}\right)^{2}}}{X} .
$$

Differentiating $I_{x}$ with respect to $X$, setting the derivative equal to zero, and solving for the current minimizing reactance, $X^{*}$, yields 


$$
\begin{aligned}
X_{t h y}^{*} & =\frac{3 V_{\max } \sqrt{n^{2} E_{b}^{2}-V_{\max }^{2}}}{P}-n X_{b} \\
& =n\left(\frac{\left.3 V_{\max } \sqrt{E_{b}^{2}-\frac{V_{\max }^{2}}{n^{2}}}-X_{b}\right)}{P}\right)
\end{aligned}
$$

with,

$$
\begin{aligned}
& I_{x}^{*}=\frac{P}{3 V_{\max }} \sqrt{1-\left(\frac{V_{\max }}{n E_{b}}\right)^{2}} \\
& I_{r}^{*}=\frac{P}{3 n E_{b}}
\end{aligned}
$$

This equation for $X_{\text {thy }}^{*}$ clearly shows that no fixed inductance can provide the same effect as the thyristors in the DMIC inverter.

The model was applied to the two motors. Figure 19 shows that providing additional dc supply voltage beyond the minimum needed to support base speed condition is beneficial in reducing motor current and therefore reducing motor copper losses. In the inverter, there may be some increase in switching and reverse recovery losses as a result of the additional supply voltage; but there will be reductions in conduction losses that are at least proportional to the reduction in current if not more. Since the conduction losses are typically the main inverter loss mechanism, the inverter losses will also decline with higher supply voltage.
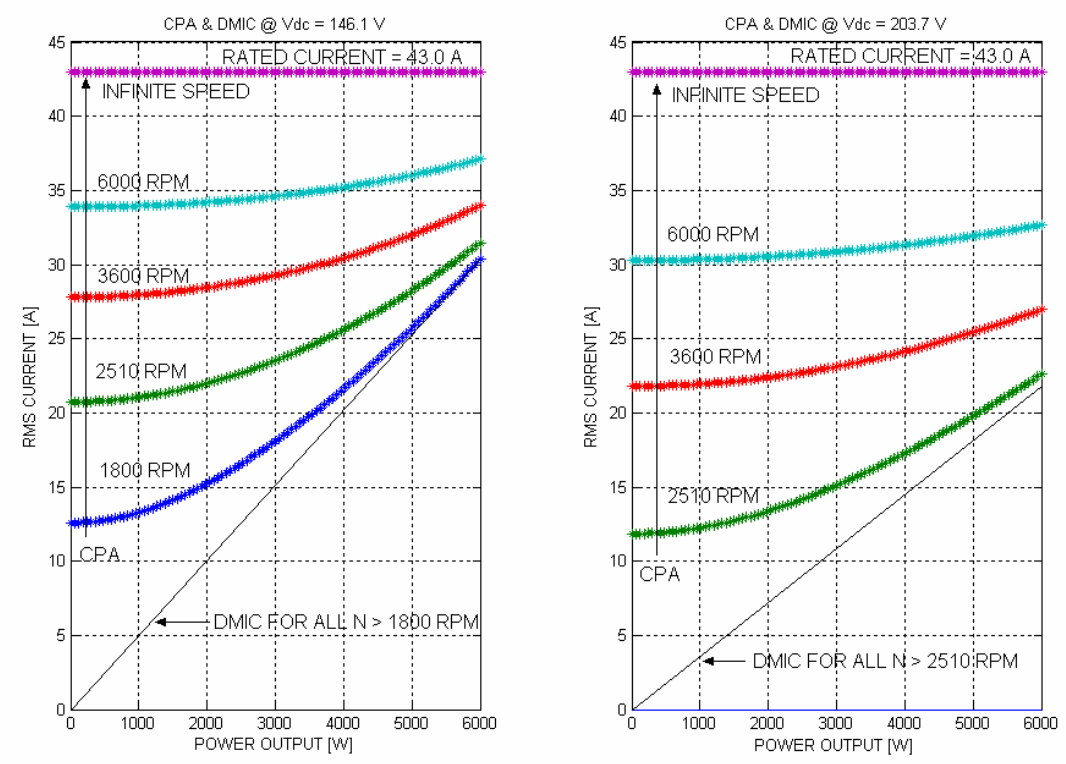

Figure 19. RMS motor current vs. power output during high-speed operation of Motor 1 with 146.1-V and 203.7-V de supply voltages, when driven by CPA or by DMIC. 
Figure 20 shows the high-speed current magnitude control capability of CPA and DMIC for Motor 1 and Motor 2 when both machines operate from a 203.8-V dc supply. Figure 20, however, shows the added benefit of having an inductance that is greater than the "optimal" value of $L_{\infty}$. Recall that the inductance of Motor 1 is exactly $L_{\infty}, 765 \mu \mathrm{H}$, while Motor 2 has significantly more inductance (1300 $\mu H$ ). Comparing the two figures, it can be observed that when the motor is driven by CPA, the higher inductance results in a lower current magnitude at any given speed and power level, thereby improving efficiency. Also note that for the high-inductance machine, the curves for the various speeds shown - 1800, 3600, and $6000 \mathrm{rpm}$ - are much closer to the performance of the DMIC-driven motor, which has a single curve describing all operating speeds greater than $1800 \mathrm{rpm}$. While the performance with CPA is closer to DMIC for a high-inductance machine, the current with the DMIC drive is always at least as low as with the CPA; therefore, DMIC should have an efficiency advantage, especially at high speed and less than full load. Although not shown here, a further increase in dc supply voltage, for example from 203.7 to $250 \mathrm{~V}$, would further reduce the motor current with DMIC but would have very modest impact on the CPA drive. Figure 20 clearly indicates that it may be very advantageous to have an inductance greater than $L_{\infty}$. Thus it is difficult to accept $L_{\infty}$ as an "optimum" design target for traction drives.
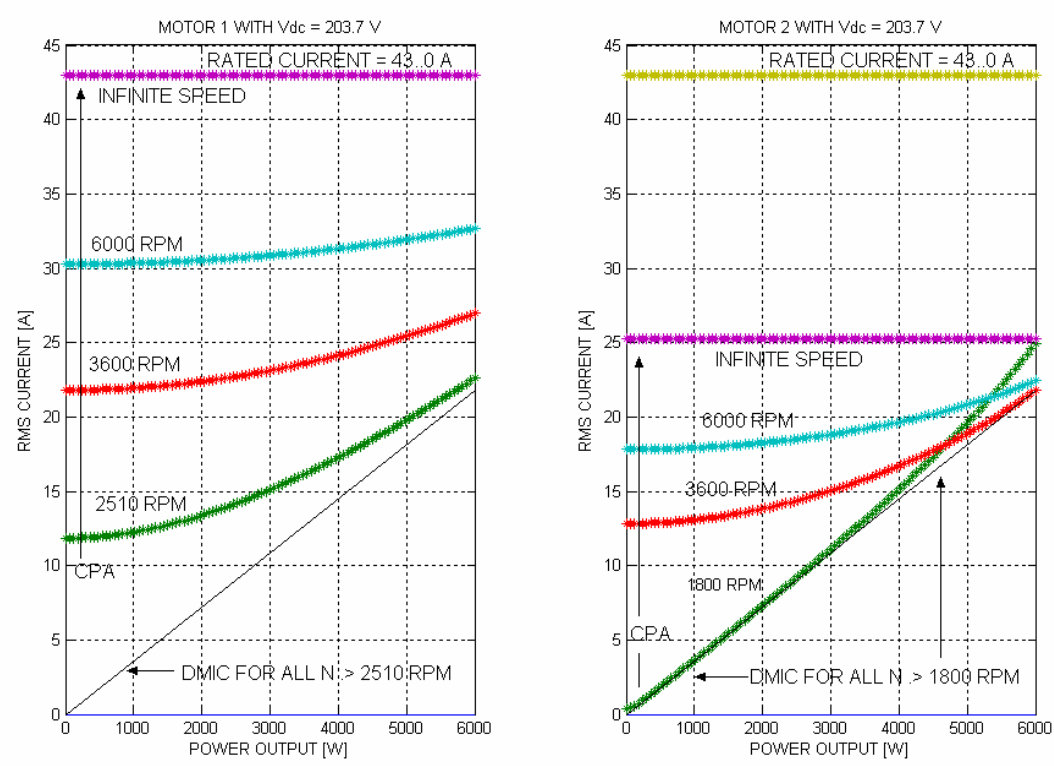

Figure 20. RMS motor current vs. power output during high-speed operation of Motor 1 and Motor 2 with $203.7 \mathrm{~V}$ de supply.

The results in this section highlight the key difference between CPA and DMIC with respect to motor current magnitude control during high-speed operation. At high speed, the rms motor currents for the two different PMSM motor control methods approach

$$
\begin{gathered}
I_{C P A}=\frac{E_{b}}{X_{b}}=\frac{E_{b}}{\Omega_{b} L} \\
I_{D M I C}=\frac{P}{3 V_{\max }}=\frac{\pi P}{3 \sqrt{2} V_{d c}} .
\end{gathered}
$$


The motor current at high speed with CPA is the characteristic current that depends only on motor parameters. If the motor inductance is sufficiently large, then the characteristic current is less than the rated current, thereby enabling operation across a wide CPSR. On the other hand, the characteristic current is independent of load, meaning that the efficiency can be poor under light-load and variable-load conditions at high speed. Since the characteristic current is independent of dc supply voltage, providing a dc supply voltage beyond that necessary to support rated torque at base speed cannot reduce the motor current at high speed.

The motor current at high speed with DMIC is proportional to load power so that good efficiency can be maintained under light-load and variable-load conditions. Also, the high-speed current is independent of machine inductance. Whether the inductance is large or small, the high-speed current will be the same. The difference in behavior between a large or small motor inductance will lie in the firing of the thyristors, which will always adjust the total inductance to minimize the rms amps per developed watt. Finally, with DMIC, the high-speed current is inversely proportional to supply voltage. Therefore, significant reduction in motor current magnitude and attendant reductions in motor and inverter losses can be achieved by providing supply voltage in excess of that required to support the specified base speed conditions.

Approximate values of the device average and rms currents can be obtained using the per-phase fundamental frequency model shown in Figure 21. The model applies for both CPA and DMIC, but $X_{t h y}$ is zero for all operating conditions when using CPA.

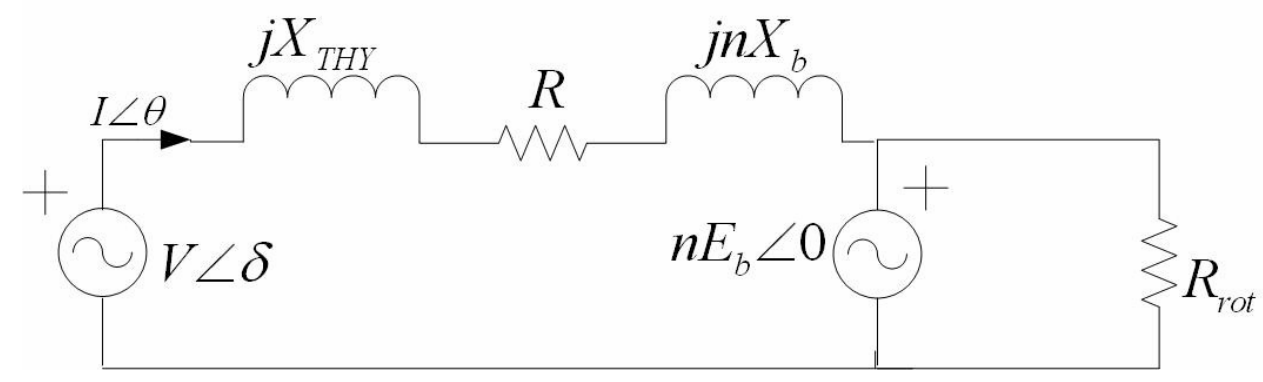

Figure 21. Per-phase model used to calculate average and rms device currents.

The model of Figure 21 explicitly represents motor copper and rotational losses since

$$
\begin{aligned}
& P_{c u}=3 I^{2} R \\
& P_{r o t}=3 \frac{\left(n E_{b}\right)^{2}}{R_{r o t}} .
\end{aligned}
$$

In this study, the winding resistance is fixed for both the example motor designs. No correction is attempted for temperature or skin effect.

Finally, we use the two motor examples to explore the benefits of having inductance higher than the optimal value, the potential benefit of providing extra dc supply voltage, the potential benefit of DMIC versus CPA, and the impact of thyristor reverse recovery losses on the performance of DMIC drives.

First we look at the benefits of inductance higher than optimal. Motor inductance is a critical factor in determining the CPSR of a PMSM when driven by CPA. For the two motors being evaluated in this study, the first has the "optimal" inductance of $765 \mu \mathrm{H}$ while the second has a much larger value, $1300 \mu \mathrm{H}$. The performance of these two motors was compared over the entire torque speed range using the fundamental frequency model. Each motor is supplied by a dc voltage sufficient to achieve rated torque, $63.7 \mathrm{Nm}$, at the specified base speed. For the low-inductance motor, the supply voltage is $151 \mathrm{~V}$; 
the higher inductance requires a 208-V supply. Performance was simulated for values of rpm from 20 to $6000 \mathrm{rpm}$ in steps of $20 \mathrm{rpm}$ and for about the same number of equally spaced load levels at each value of $\mathrm{rpm}$. Since the speed increment was 20/6000 of full speed, the load increment was chosen to be 25/6000 of full load. Results of the simulations are shown in Figures 22 through 25. The plots are 3-dimensional renderings with shading. The colors used in the shading denote magnitude. The highest value is red, the lowest value is dark blue. The transition from high value to low value is red to yellow to green to aqua to blue. The $\mathrm{x}$-axis of each plot is rpm from 0 to 6000, while the $\mathrm{y}$-axis is torque from 0 to $63.7 \mathrm{Nm}$. The $\mathrm{z}$ axis variable displayed is the efficiency difference, because it lucidly exposes regions where one system performs better than another one.

Based on the comparisons in Figure 22. it is seen that the optimal characteristic inductance for field weakening does not lead to performance superior to that of Motor 2, which has 1.7 times as much inductance, when both motors are driven by CPA. The characteristic current of Motor 2 is lower than for Motor 1. Since the machine current approaches the characteristic current at high speed, there is a definite advantage to being able to operate at low current. So long as each machine is given sufficient dc supply voltage to support rated torque at base speed, the higher-inductance machine will have greater efficiency at high speed.
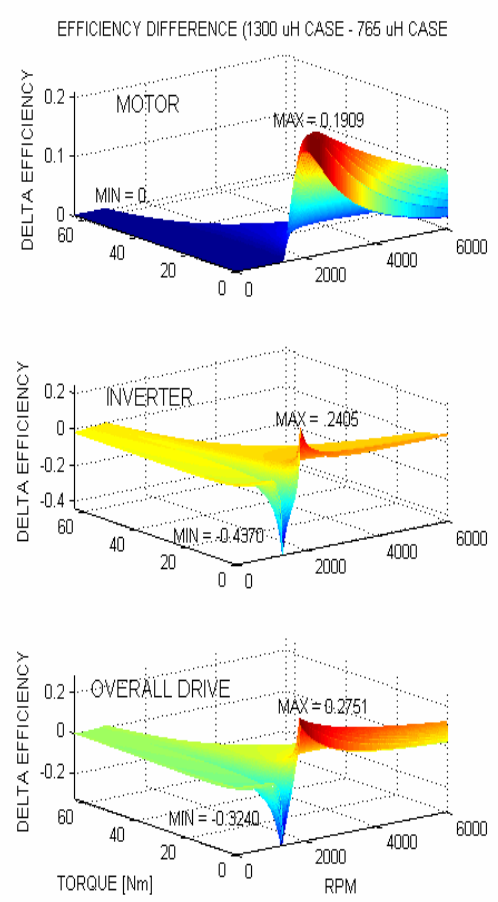

Figure 22. Efficiency difference comparison of Motor 2 (with $208 \mathrm{~V}$ dc supply voltage) minus Motor 1 (with $151 \mathrm{~V}$ dc supply voltage) when driven by CPA over the full torque speed envelope.

Next we consider the impact of having a dc supply voltage larger than the minimum required to support rated torque at base speed, which can be considered a form of "cheating" to achieve CPSR objectives. Any motor having an inductance higher than the "optimal" value of $L_{\infty}$ has an infinite CPSR. Therefore, any increase in supply voltage above the minimum would not provide improved CPSR but might impact motor current and efficiency. In Figure 22, the performance of Motor 1 and Motor 2 were compared, with both machines having the minimum dc supply voltage-151 V for Motor 1 and $208 \mathrm{~V}$ for Motor 2-to support rated torque at base speed. Since the only difference in these two machines is the 
inductance, it is reasonable to infer that Motor 1 might benefit from the higher voltage that would inherently be required by the parameter of Motor 2. The performance of Motor 1 was simulated over the entire torque-speed envelope for these two supply conditions, and the results are shown in Figure 23.

Figure 23 shows the efficiency of the lower-voltage case subtracted from the higher-voltage case at each torque-speed operating condition. This is done for the motor, inverter, and overall drive efficiency. The figure shows that there is a substantial benefit to the motor efficiency when operating at high speed. The benefit is the result of the fact that the minimum rms motor current is reduced by the additional supply voltage whether winding resistance is included or neglected. The motor efficiency at high speed is increased by as much as 0.1909 during high-speed operating conditions. There is a small region in the vicinity of base speed with low load where the lower voltage results in greater efficiency. But for highspeed operation, the additional dc supply voltage improves efficiency.
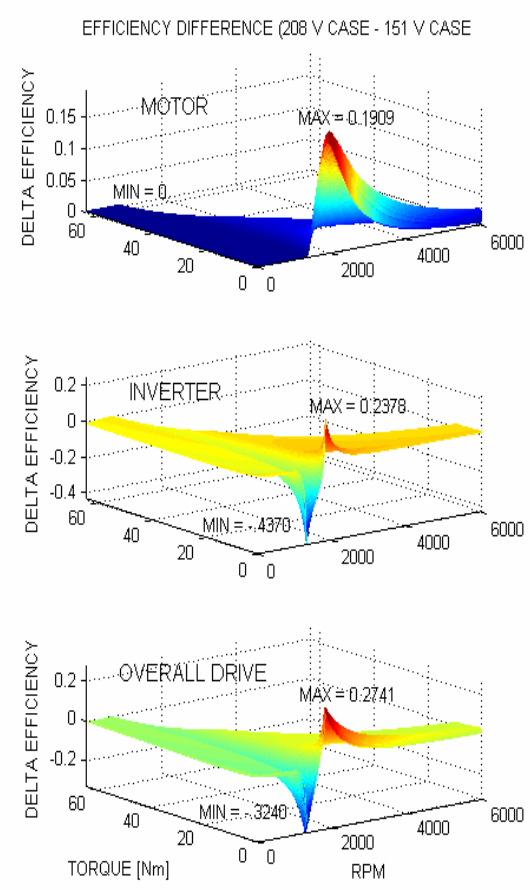

Figure 23. Comparison of efficiency difference when Motor $1 \mathrm{dc}$ supply voltage is raised from 151 to $208 \mathrm{~V}$.

Figure 24 contains simulation results over the entire torque-speed envelope for Motor 1 when driven by CPA and by DMIC. In both cases, the dc supply voltage is $151 \mathrm{~V}$, which is the minimum necessary to support rated torque at base speed. The field weakening component of current in the DMIC drive is proportional to the load, as is the rms current. In the CPA drive, the rms current approaches the characteristic current. Since the torque-producing current decreases with speed and is identical for both the CPA and DMIC cases, the field-weakening current tends to the characteristic current at high speed. Because the rms current in the DMIC drive is proportional to the load, this drive can maintain high motor efficiency at high speed. At high speed, and especially at light load, the motor, inverter, and total losses are lower with DMIC. At low speed, the conduction losses of the DMIC thyristors result in greater inverter and overall losses. At high speed, the inverter losses of the DMIC, including the thyristors, are lower because of the high speed current minimization of DMIC. These effects are also seen in Figure 24, in which the motor, inverter, and overall efficiency of the CPA case are subtracted from the corresponding DMIC case over all torque-speed conditions. The motor is the main beneficiary of DMIC; because of the lower current at high speed, the motor efficiency is higher by as much as 0.2420 . The 
inverter efficiency is lower at and below base speed because of the added thyristor conduction losses, but the inverter efficiency is higher during high-speed operation by as much as 0.1375 . Regarding overall efficiency, the DMIC may be as much as 0.0549 lower than the CPA during low-speed operation and as much as 0.2685 higher while operating in the constant power mode. If the drive spends most of its operating time at or above base speed, then the efficiency of the DMIC may be significantly better than CPA when inverter-grade thyristors are used in the DMIC drive.

Finally, we investigate the impact of converter- versus inverter-grade thyristors in the DMIC drive. Converter-grade thyristors are cheaper but have higher reverse recovery losses. Inverter-grade thyristors cost more but have lower losses and higher switching speed. For Motor 1 with 151-V dc supply, the difference in inverter efficiency and total efficiency is shown in Figure 25. Below base speed, the two cases are nearly identical. Above base speed, the reverse recovery losses of the converter-grade thyristor are larger than those of the inverter-grade. Figure 25 shows that the inverter-grade thyristor may have an

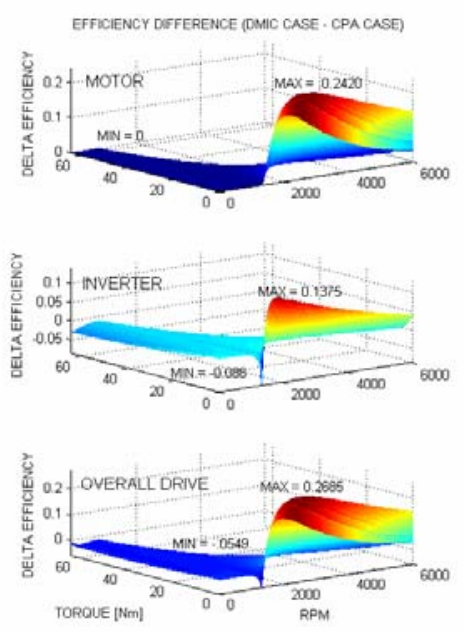

Figure 24. Comparison of efficiency difference when Motor 1 is driven by DMIC and by CPA using invertergrade thyristors from a 151-V dc supply voltage.
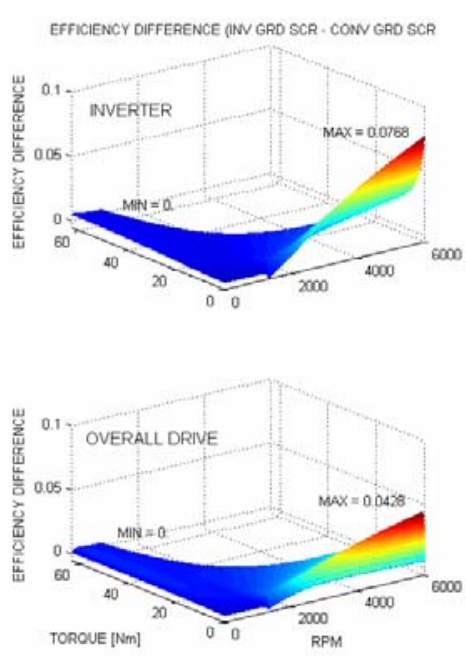

Figure 25. Efficiency difference between inverterand converter-grade SCRs for Motor 1 driven by DMIC from a 151-V de supply voltage. 
efficiency that is at most 0.0768 larger than for the converter-grade. The figure also shows that the total efficiency with inverter-grade thyristors is as much as 0.0428 larger than for converter-grade. These differences occur at $6000 \mathrm{rpm}$, where the fundamental frequency is $1.5 \mathrm{kHz}$. Although the thyristor commutation in the DMIC occurs at natural current zero crossings, it is not clear whether converter-grade thyristors are even capable of such a switching rate. The authors have done testing with converter-grade thyristors in a DMIC drive operating at $300 \mathrm{~Hz}$, and performance was satisfactory. The motors under investigation have 30 poles, which is the cause of their high fundamental rate. At this point, it is not known whether converter-grade thrysistors, which are cheaper, have switching frequencies sufficiently high to drive high-pole-count PMSMs.

\section{Reluctance Control}

International technology has focused research on the IPM motor to produce an HEV that will capture a large share of the world market. One of the beneficial features of this technology is the additional torque produced by reluctance. The objective of this task is to analytically describe the role that reluctance plays in PM motors, to explore ways to increase reluctance torque without sacrificing the torque produced by the PMs, and to compare three IPM configurations with respect to torque, power, amount of magnet material required (cost), and percentage of reluctance torque. Results of this comparison will be used to determine the research direction to obtain maximum torque and power while using a minimum amount of magnet material.

Since locomotion applications tend to prefer motors with wide ranges of speed to eliminate the need for or reduce the complexity of gear-boxes, it is important to find ways to increase the speed range of PM motors. One obvious possibility is to use a multilevel voltage source where available voltage would increase with rotor speed. Inverter drive costs, which are already the major component of a PM drive system, may limit this approach to two voltage stages, with the higher stage enabled at the higher speeds. Presently, improvements in stator and rotor topologies, together with control approaches, have enabled better overall performance/cost potential.

Once a PM machine is built, the strength and number of magnets in the rotor, and the number of poles and turns in the coils of the stator, traditionally remain constant; thus the amount of PM-generated magnetic flux linked by the coils of the stator remains fixed. As a result, the back-emf voltage induced by the PMs increases linearly with the speed of the rotor.

Rotor speed increases the back-emf voltage and thus causes a rapid reduction in the available supply voltage, which is normally constant. When there is no longer any supply voltage available to drive current into the stator, the maximum speed has been reached.

Reluctance is a property used in magnetic circuits that accounts for the ratio of geometric length to area and the magnetic properties of a medium, such as permeability, $\mu$, in which a magnetic flux flows. The expression for reluctance is $\mathcal{R}=l /(\mu A)$, where $l$ is the length of the flux path and $A$ is the area normal to the flux [36]. Reluctance relates magnetic flux to mmf in a manner similar to the way that resistance and inductance relate current to voltage in an electric circuit. Since the externally controllable and measurement parameters of a motor are electric, it is customary to study the performance of motors in terms of electric rather than magnetic quantities. The relationship between magnetic reluctance and electric inductance is complex except for the simplest of cases, like that of a coil wrapped with $N_{t}$ turns of wire around a magnetic path of reluctance, $\mathcal{R}$. This simple relationship is $\mathrm{L}=N_{t}^{2} / \mathcal{R}$, which shows that electric inductance is proportional to the inverse of magnetic reactance. Consequently, high inductance corresponds to low reluctance.

Next is an analysis showing the relative benefit of flux weakening and saliency. In PM motors, optimal torque production occurs when the back-emf voltage and the stator current are in phase. When they are not, the current component normal to the back-emf produces a magnetic field that opposes or reinforces that of the PM, depending on its direction. The time-dependent linked flux then includes stator reaction components in both the $\mathrm{d}$ - and the q-axes, such that 


$$
\begin{gathered}
\lambda_{\mathrm{d}}=\lambda_{\mathrm{pm}}+\lambda_{\mathrm{rd}}=\lambda_{\mathrm{pm}}+L_{d} i_{d} \\
\lambda_{\mathrm{q}}=\lambda_{\mathrm{rq}}=L_{q} i_{q},
\end{gathered}
$$

where

$i_{q}$ is the current component in-phase with the PM's back-emf (q-axis direction), which is the primary torque-producing component of current,

$i_{d}$ is the current component normal to the back-emf (d-axis direction), which is the flux-producing component of current,

$\lambda_{r d}=L_{d} i_{d}$ represents the flux linkages created by the stator current's $i_{d}$ component,

$\lambda_{r q}=L_{d} i_{d}$ represents the flux linkages created by the stator current's $i_{q}$ component,

$L_{d}$ and $L_{q}$ are the inductances when the rotor is in the d-axis and q-axis positions.

Equation (15) shows that when $i_{d}$ is negative, the reaction field has a d-axis component opposing that of the PM, thereby weakening the flux. This in turn causes a reduction in the q-axis back-emf voltage; consequently, higher $I_{q}$ currents at the same or higher speeds for the same total current are possible, thus extending the motor's operating speed range. This extension is achieved at the cost of additional ohmic losses in the stator, but it reduces the angle, $\alpha$, between the stator's voltage, $V$, and current, $I$, thus improving the power factor.

Figure 26 shows the RMS phasor diagram describing the operation of a generic PM motor driven sinusoidally with flux-weakening, since the current is in the negative d-axis direction.

As discussed in the caption of Figure 26, in addition to flux weakening, extra mechanical power is produced when the saliency ratio, defined as

$$
\xi=L_{q} / L_{d}
$$

is larger than unity. In surface-mounted PM motors characterized by $\xi=1$, the stator reaction back-emf, $E_{r}$, is perpendicular to the stator current, $I$, and thus their vector product is zero. Hence, in surfacemounted PMs, $I_{d}$ has no effect in power production, although it increases the ohmic losses and improves the power factor.

A discussion of characteristic current follows. The magnitude of the d-axis current needed to completely cancel the PM magnetic flux is often referred to as the motor's "characteristic current." In the open literature, the characteristic current is also identified as $I_{x}$ [12]. By setting $\lambda_{\mathrm{d}}=0$, in Eq. (19) we obtain

$$
i_{c h}=\left|-i_{d}\right|=\lambda_{p m} / L_{d}
$$

Surface-mounted PMs, for which $L_{q}=L_{d}$, when operated with an amount $I_{c h}$ of d-axis current in the negative direction would have no speed limit, since the back-emf induced by the PM would be zero. Unfortunately, the inductance, $L_{d}$, is small in PM motors; consequently, the magnitude of the characteristic current is large, showing that large currents cause only small amounts of flux weakening.

The power output for a PM with an $I_{d}$ equal to the characteristic current, $I_{c h}$, in Eq. (21) injected in the negative direction would be

$$
\text { Pout }_{c h}=I_{q}\left(E_{p m}+\left(\lambda_{p m} / L_{d}\right)\left(L_{q}-L_{d}\right) \omega\right)=I_{q} E_{p m} L_{q} / L_{d}=I_{q} E_{p m} \xi
$$



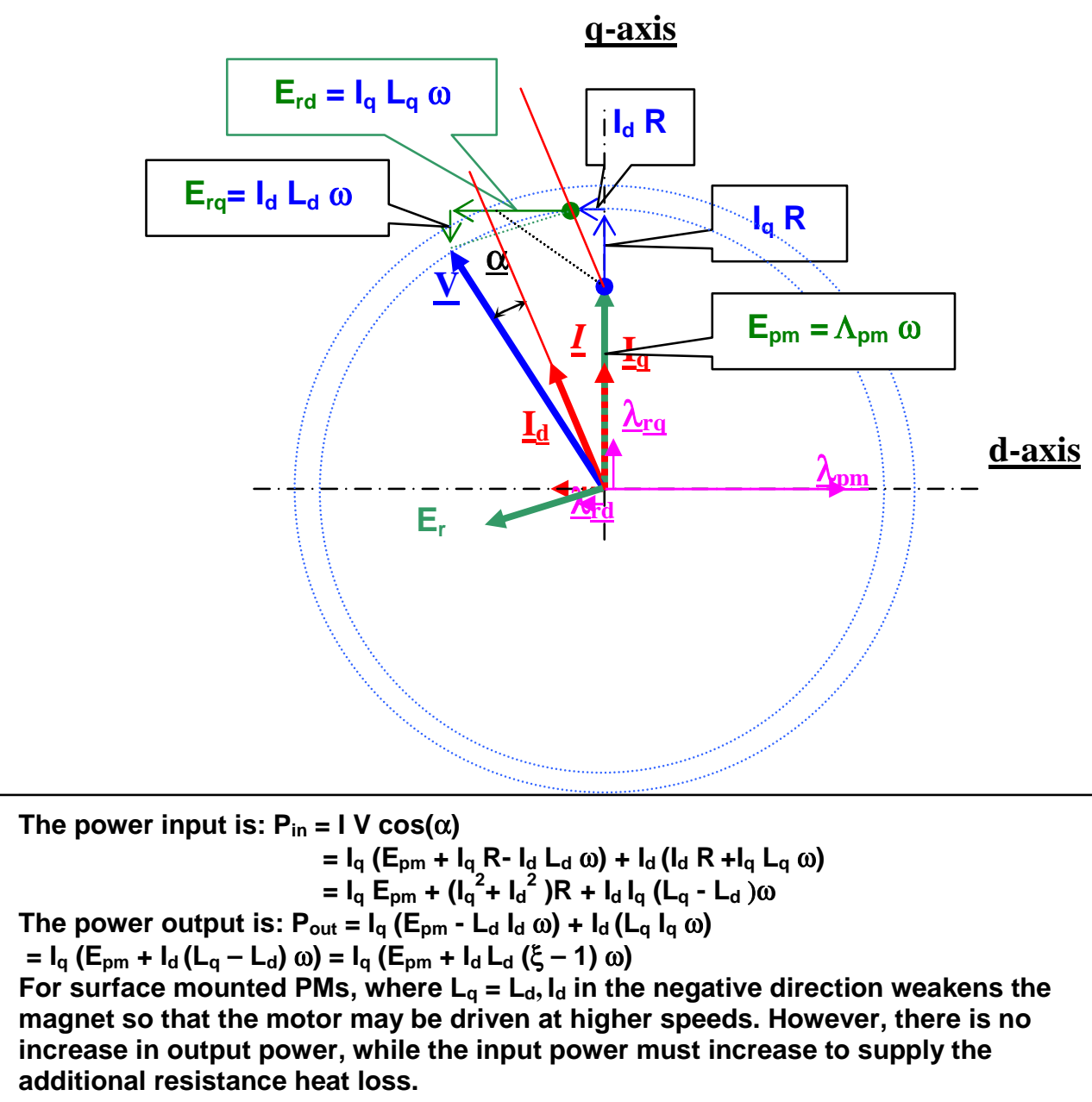

Figure 26. Standard RMS phasor diagram of a PM motor with flux weakening.

IPM motors differ in that they have the PMs inserted in the body of the rotor. This not only provides mechanical support for the magnets and protects them from the environment and from demagnetization, but also makes the inductance of the rotor position dependent, so that $L_{d}<L_{q}$. Consequently, when an $I_{d}$ current is inserted in the negative direction shown in Figure 26 to weaken the flux, extra mechanical power is generated in the motor. IPMs are thus considered as hybrids of PM and reluctance technologies. In addition, in IPMs, the boundary between the rotor poles and the air-gap is high-permeability silicon steel. It allows phase-advance to give the PM flux a tangential component within the rotor pole, thus skewing the air-gap flux distribution to such a degree that stator teeth are saturated. This allows for an externally controlled variation in reluctance that is not possible in SPM motors.

As shown in the caption of Figure 26, the power output for any PM is

$$
P_{\text {out }}=I_{q}\left(E_{p m}+I_{d}\left(L_{q}-L_{d}\right) \omega\right)=I_{q}\left(E_{p m}+I_{d} L_{d} \omega(\xi-1)\right) .
$$

Thus the torque expression,

$$
T=P_{\text {oul }} / \omega=I_{q} E_{p m} / \omega+I_{d} I_{q}\left(L_{q}-L_{d}\right)=\text { Torque }_{P M}+\text { Torque }_{\text {Reluctance }},
$$

has two components. In IPMs, the reluctance term is not zero and contributes to the motor's output. 
Equation (24) shows that the total torque output can be maintained while reducing the PM torque by compensating with an increase in the reluctance torque. When the strength of the PM flux is reduced, $\lambda_{p m}$ and the magnitude of the characteristic current are also reduced. This makes flux-weakening by insertion of negative current in the d-axis less costly and more practical.

Solving Eq. (24) for $E_{p m}$, substituting $E_{p m}=\Lambda_{p m} \omega$, and taking the partial derivative of $\Lambda_{p m}$ with respect to $\xi$ for fixed $P_{\text {out }}, \omega, I_{d}, I_{q}$, and $L_{d}$, we obtain the relationship between the change of PM strength needed and a change in the degree of saliency,

$$
\delta \lambda_{p m}=-I_{d} L_{d} \delta \xi
$$

That is, an increase of saliency would result in a decrease in magnet strength required proportional to the product of $I_{d} \bullet L_{d}$, the values of the d-axis inductance and d-axis current component.

In terms of the characteristic current, Eq. (25) becomes

$$
\delta \lambda_{p m} / \lambda_{p m}=-I_{d} / I_{c h} \delta \xi
$$

and integration yields

$$
\lambda_{p m}=k e^{-I d / I c h \xi},
$$

where $k$ is an integration constant. This is an important relationship since it shows that the magnet strength needed diminishes exponentially with the degree of saliency and with the amount of d-axis current injected relative to the value of the characteristic current. Therefore, decreasing the value of the characteristic current and increasing the saliency are clear goals for improving PM motor performance.

Equations (19) through (27) provide the following insights.

(a) Equation (21) shows that to reduce the magnitude of a motor's characteristic current, one may reduce the magnitude of the PM flux linked, and/or increase the value of the d-axis inductance.

(b) Equation (23) shows that IPM motors are superior to surface-mounted motors for flux weakening, since under flux-weakening conditions they provide extra mechanical power output because their saliency is larger than unity.

(c) Equation (25) shows the desirability of increasing the saliency and the d-axis inductance in order to reduce the amount of PM flux linkage needed by a motor. This would further enhance the motor's flux-weakening capabilities and reduce the cost of PMs while maintaining the motor's power level.

(d) Equation (27) shows that for equal motor power output, the magnet strength needed diminishes exponentially with the degree of saliency and with the amount of d-axis current injected relative to the value of the characteristic current. Therefore, decreasing the value of the characteristic current and increasing the saliency should be clear goals for improving PM motor performance.

(e) Equation (20) shows that to increase the saliency ratio, $\xi$, one should increase the quadrature inductance, $L_{q}$. One could decrease the d-axis inductance, $L_{d}$, but it would be counterproductive because of (a) and (c), which call for an increase in $L_{d}$.

If $L_{d}$ is increased as recommended by (a) and (c) above, then a proportional or larger increase should be sought for $L_{q}$ in order to maintain or augment the saliency ratio as recommended by (d).

We have investigated three PM motors that exhibit varying amounts of reluctance. The inset PM was assessed for the impact of trading PM for iron; the V-shaped single-layer IPM was assessed for the impact of embedding the PMs in the iron and of trading PM for iron; and the U-shaped multilayered IPM was assessed for the effect of changing the amount of PM material in an embedded PM design. For each design, the performance vs amount of PM material was studied to determine the optimal configuration. Parametric computations of performance were performed using the SPEED Consortium's brushless dc 
computer simulator for each design. The topologies, methodologies, and results obtained are presented and discussed in the next section.

Three IPM motor configurations, which exhibit different reluctance properties, have been parametrically characterized for comparison of torque, power, and amount of magnet material. Figures 27 through 29 show two variations of their structures with two different distributions of PM and iron.

Figures 27(a) and 27(b) correspond to the inset type motor, whose magnets are flush with the rotor surface. In the parametric calculations for this motor, the angular pitch, $\beta_{\mathrm{M}}$, of the PM is varied from a low value of $5^{\circ}$ to a high value of $175^{\circ}$ in $10^{\circ}$ increments. The degrees are with respect to one complete electrical cycle, which includes a north and a south pole. In Figure $27(\mathrm{a})$, one quadrant represents a $360^{\circ}$ electrical cycle, which includes 12 stator teeth. The angular pitch, $\beta_{\mathrm{M}}$, is the angle in electrical degrees subtended by the magnet. In Figure 27(a), this includes four teeth, which leads to $360^{\circ} \times 4 / 12=120^{\circ}$. Note that as the pitch of the magnets decreases, the width of the iron web between the magnets increases. Also note that for the highest pitch, the expected behavior is that of an SPM motor.

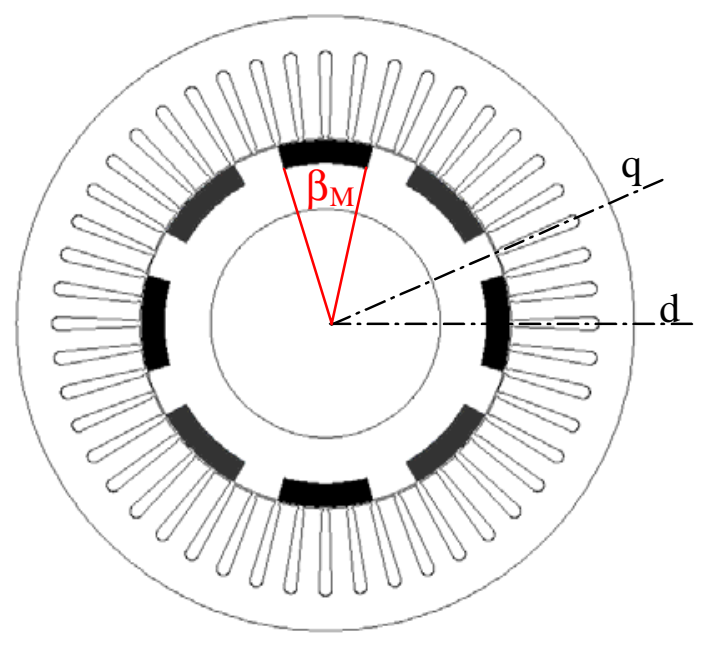

(a). Low magnet fraction, $\beta_{M}=120^{\circ}$.

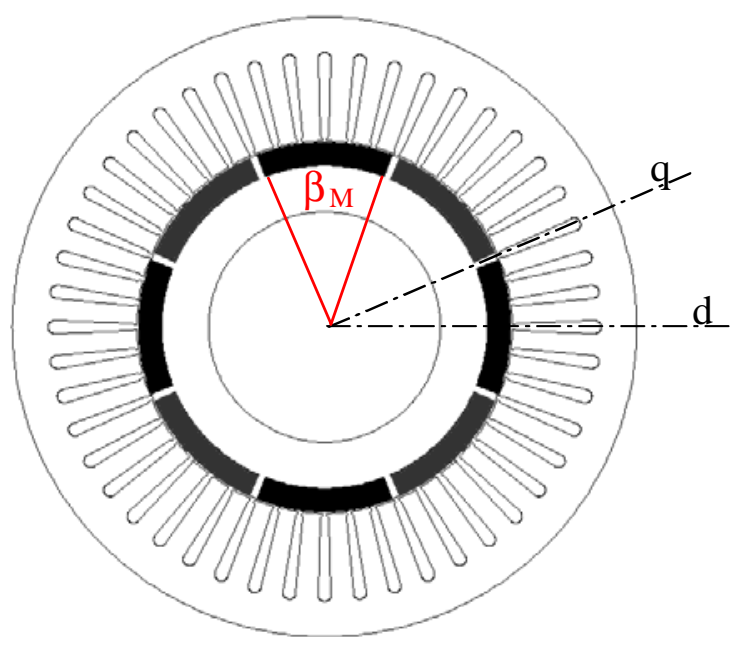

(b). High magnet fraction, $\beta_{M}=175^{\circ}$.

Figure 27. Inset surface-mounted PM motors.

Figures 28(a) and 28(b) correspond to the V-shaped IPM motor. In the parametric calculations for this motor, the lower radial position of the PMs is kept constant while the angular pitch of the PM at the rotor's surface is varied from a low value of $50^{\circ}$ to a high value of $175^{\circ}$ in $10^{\circ}$ increments. Note that as the pitch of the magnets decreases, the width of the iron web between the magnets increases. In this design, we have reluctance variations due to the iron web, as in the inset type motor; but in addition we have the soft-PM effect of iron pole-caps that allow control of the shape of the distribution of flux crossing the air-gap.

Figures 29(a) and 29(b) correspond to the dual-layer U-shaped IPM motor. In the parametric calculations for this motor, the angular pitch of the PM at the rotor's surface and the lower radial position of the PMs are kept constant, while the length of the lower magnets in the two layers of U-shaped PMs is varied proportionally. The length of the innermost layer's magnet, yI_1, is varied from a low value of $2.4 \mathrm{~mm}$ to a high value of $17 \mathrm{~mm}$ in $0.73-\mathrm{mm}$ increments. Note that the width of the iron web at the airgap boundary remains constant, while the amount of magnetic material increases with the value of yI_1.

Each motor topology was explored using the brushless dc motor simulation software, a product of the SPEED Consortium. All of the motors have the same stator and overall rotor dimensions. The same operating conditions, such as max current and voltage, were used for all to provide the basis for comparative analysis. 

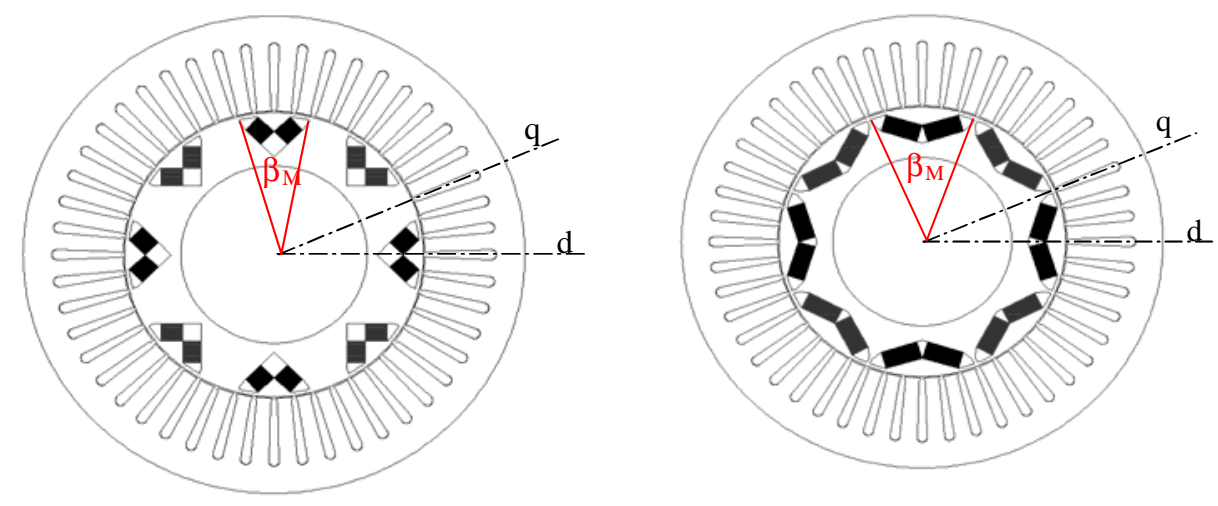

(a). Low magnet fraction, $\beta_{M}=50^{\circ}$. (b). High magnet fraction, $\beta_{M}=160^{\circ}$.

Figure 28. V-shaped interior PM motors (type 4).

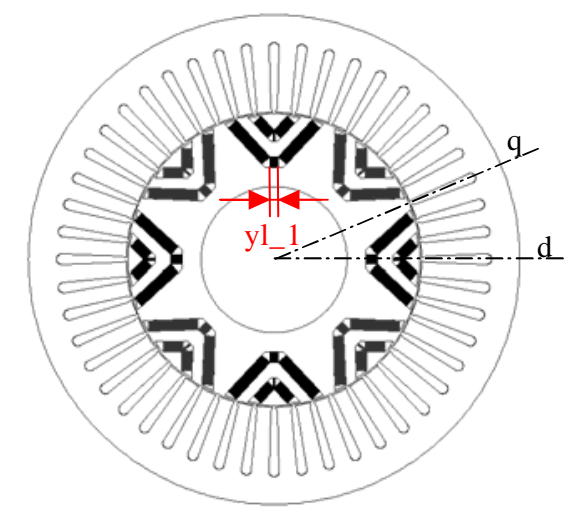

(a). Lowest magnet fraction, yI_1=2.4 mm.

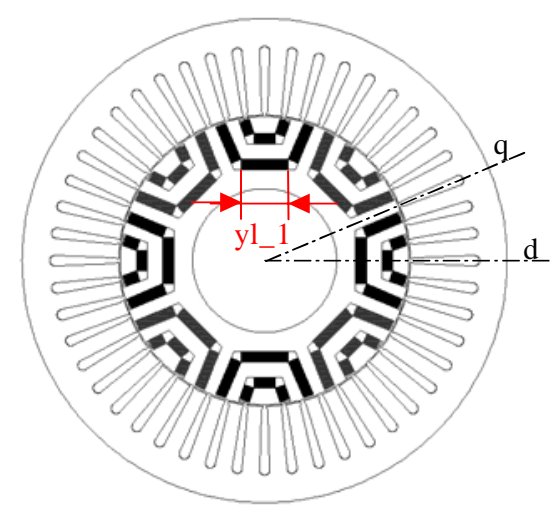

(b). Highest magnet fraction, $\mathbf{y I} \_1=17 \mathrm{~mm}$.

Figure 29. U-shaped interior PM motors (type 6).

The simulation software computed the PM and reluctance torques, power output, current peak and RMS, back-emf, and so on, characterizing the performance of the motors. The analysis excluded thermal flux and mechanical stress analysis, although these would be of interest for further study.

For each of the three topologies, parametric studies were performed that bracketed a range of advance angles within a range of speeds for each magnet configuration. The magnet-to-iron ratio was varied by means of the PM angular pitch parameters, $\beta_{\mathrm{M}}$ or $\mathrm{yI} \_1$, as appropriate.

Figure 30 shows how the amount of PM material varies with the varying parameter, $\beta_{\mathrm{M}}$ or yI_1, in each motor type. Note that Beta $\mathrm{M}=\beta_{\mathrm{M}}$ in the figure. There is a large difference between the three topologies. At each speed, there is an optimal phase advance, which determines the flux-weakening current component, for maximum torque production. This phase advance is not necessarily the same for optimum motor efficiency. The results presented here correspond to the phase advance that resulted in the highest torque.

Figure 31 for the inset PM motor is of interest because it shows that there is a magnet fraction of about $115 / 180=0.64$ for which about $75 \%$ of the torque is reluctance torque over the whole speed range. This is a $36 \%$ reduction in magnet area, which could amount to a cost savings if the thickness of the magnet does not have to be increased to maintain required PM performance. 


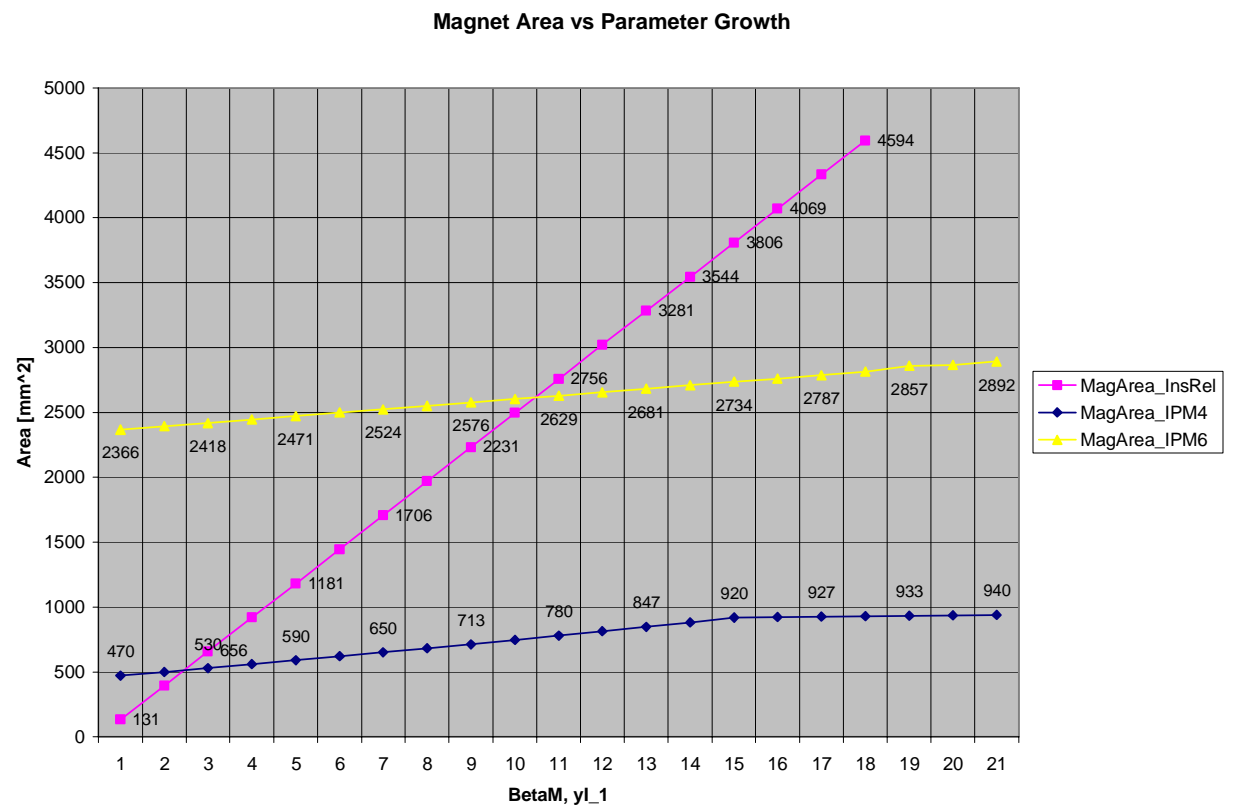

Figure 30. Amount of PM material in terms of cross-sectional surface area as a function of $\beta_{\mathrm{M}}$ or $y \mathrm{yl}_{-} 1$ for each of the three rotor configurations.

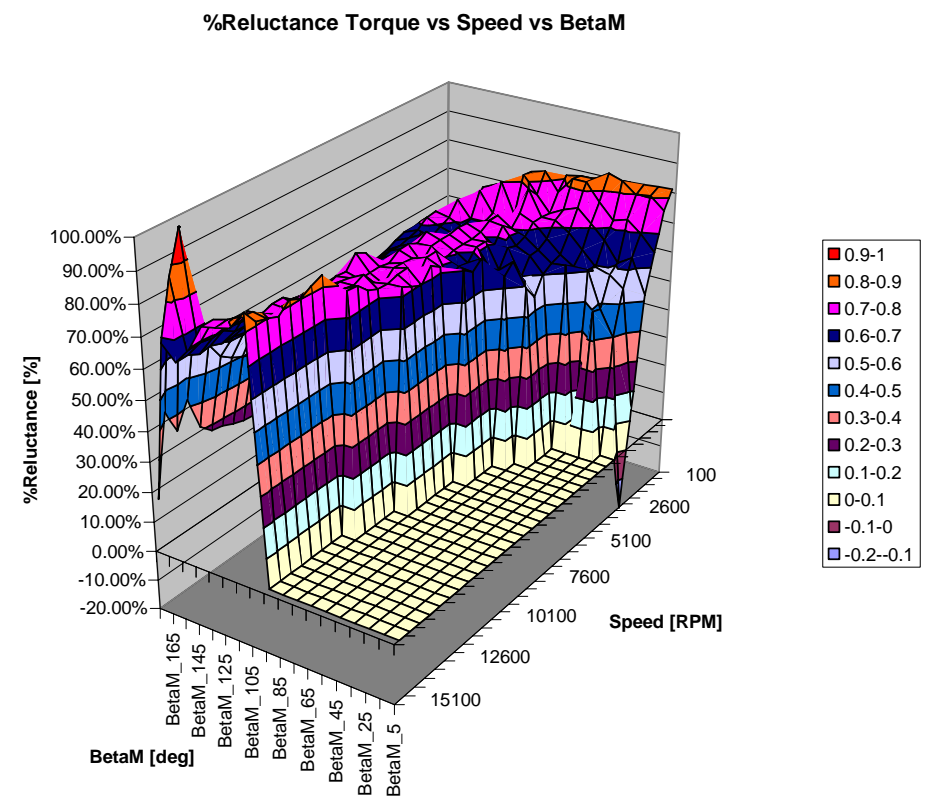

Figure 31. Relationship between magnet pitch, $\beta_{M}$, speed, and percentage of reluctance torque for the inset PM motor.

The reluctance torque has its strongest contribution in the current limited region below the base speed where maximum torque is required. Thanks to the reluctance contribution, higher peak torques can be achieved at low speeds for the same current limit. This suggests that to improve the performance of a PM motor, one could reduce the magnitude of the PM energy in the rotor, a radical thought, by reducing the PM mass or its magnetic strength and increasing the rotor's saliency to compensate the loss in PM torque with increased reluctance torque. In turn, the reduction in PM energy results in a reduced rate of change in 
the back-emf and magnetic losses as the speed increases. This increases the speed range, improves the efficiency, and reduces the motor's heat removal load. The impact on power output and efficiency from changing the amount of magnetic material and its magnetic strength in a PM motor depends also on the control strategy used. ORNL has investigated the impact of trading PM material for iron in an inset PM controlled with the optimal displacement angles for each speed and PM material geometric and magnetic characteristics.

This task was initiated because of Figure 32. The reluctance torque is represented by the distance between the total torque and the PM torque produced by a four-pole motor as the magnet fraction is varied from 0 to 180 electrical degrees. Figure 32 shows that the total torque passes through a maximum at a magnet fraction of about $3 / 4$, suggesting that there is a magnet fraction at which the torque is optimum. Furthermore, at that point there is a significant increase in the torque above that produced by the magnets alone. At the onset of this task it was thought that the primary benefit of a reluctance-assisted PM motor was that it could achieve a required torque with less magnet material, which would allow it to operate at a higher CPSR. Alternately, it can achieve a higher torque than an SPM motor.

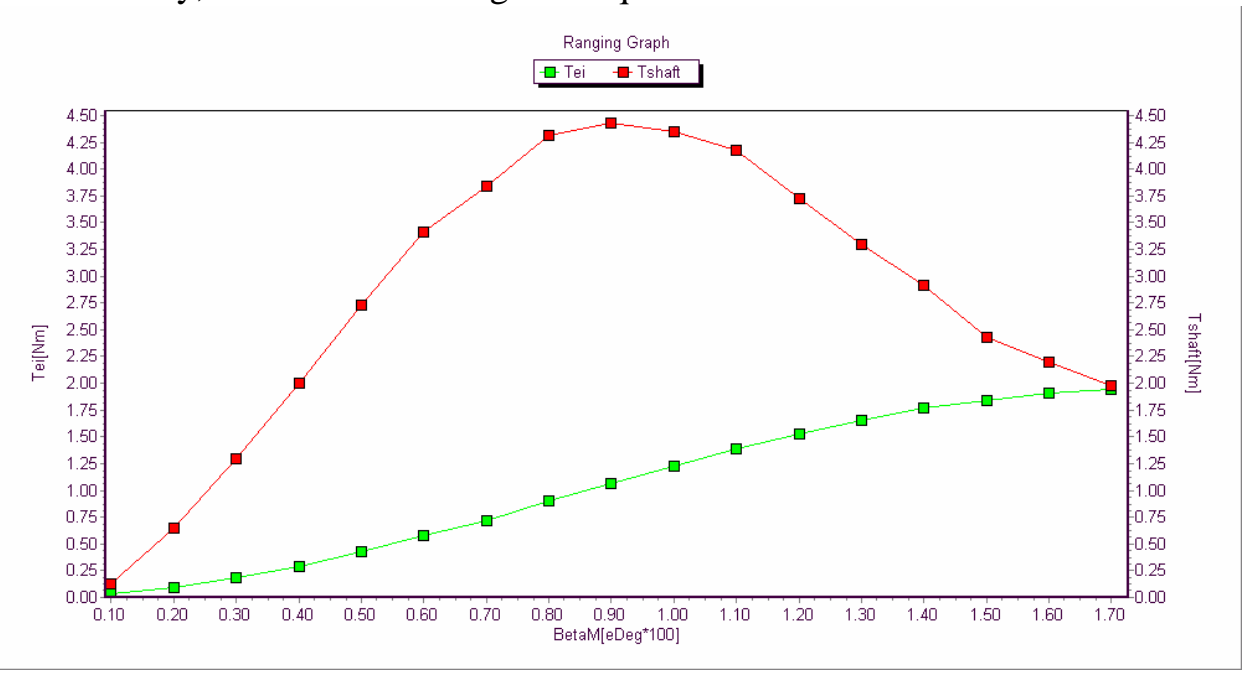

Figure 32. Magnet torque and total shaft torque of a four-pole inset reluctance-assisted PM motor for a range of magnet fractions.

The rest of this effort was to examine the equations used to design IPMs and to determine the optimal magnet fraction.

The use of lumped-parameter equations for modeling is appealing because it facilitates understanding, but it requires the determination of inductances, resistance, and flux linkages with the degree of detail appropriate for the simulation goals. Most challenging is the characterization of magnetic saturation since, especially in the case of IPM motors, the magnetic flux paths vary significantly with the rotor's relative position to the stator-generated rotating field and with the magnitude of the stators' currents.

It is best to have measured values of the lumped parameters, and second best is to obtain them by means of finite element computations. Most often, though, they are obtained by means of calculations based on geometry, equivalent circuits, and adjustment factors.

The focus of performance can be on power output or efficiency and may flip from one to the other depending on the situation. For automotive applications, it seems appropriate to focus on efficiency; but then one needs to consider the speed-torque cycle for each particular application. We consider that, in addition to the standard efficiency/torque maps, the overall efficiency for a set of standard driving cycles could be a criterion to determine optimal configurations, which, in our case, is for the optimal reluctanceassisted PM configuration. 


\section{Conclusions}

\section{Voltage Control}

- The Z-source inverter is an excellent FCV technology.

- Its present configuration does not migrate naturally into conventional ICE-powered HEV configurations. The problem is that the Z-source inverter's front end diode, which is important in its operating cycle, is either an obstacle to regeneration or an unnecessary voltage drop in ICE configurations.

- Although there is some objection to the Z-source inverter's diode, even in an FCV, for applications that require inverter efficiencies above 98\% [37], a recent report [22] has shown that the Z-source inverter is $1 \%$ better than a traditional PWM inverter, thereby meeting the $97 \%$ FreedomCAR efficiency specification. Further advantages include $20 \%$ lower semiconductor ratings and a CPSR greater by a factor of 1.68 than traditional PWM inverters. An inherent benefit is higher reliability because it is not subject to damage from shoot-through.

- The report [22] also explains an additional self-boost mode discovered during testing, which comes into play when the traction motor is operating at low speed, low power, and low power factor. This mode is necessary to successfully start a cold fuel cell and is controllable when there is a battery, required by all FCVs, in the inverter system; however, it could be a problem for other applications.

\section{Flux Control}

- Conventional vector control maintains a torque-producing quadrature current, $\mathrm{i}_{\mathrm{q}}$, and a magnet field-weakening $d$-axis current, $i_{\mathrm{d}}$. The component of the $\mathrm{d}$-axis current must be maintained during operation at high speed and results in increased stator current resistance losses with an accompanying loss in efficiency. Vector control technology is well established and allows CPSR extension by flux weakening. It does require an expensive absolute encoder and fast control algorithms.

- In general, efficiency considerations make those methods of flux control that require temporary intervention or pulses preferable to those requiring continuous action during operation.

- The most attractive method of real time flux control (RTFC) is the pole number change. This has potential to enhance vector control because it would perform the same current decomposition to form a d-axis current pulse that would demagnetize magnet(s) as operation at high speed begins and re-magnetize magnet(s) as high-speed operation ceases.

- Preliminary studies have shown that as the speed of a PM motor increases, the number of turns per coil must be reduced to optimize power delivery. Reducing the number of turns per coil is also a method of reducing the back-emf to accomplish RTFC. Benefits include additional power as well as increased CPSR.

- Another attractive method of RTFC is changing the number of coils, which would have to be used in conjunction with the pole number change because the ratio of coils to poles must remain constant. A number of papers have discussed changing the coil number for an induction motor, which automatically induces fewer poles when the number of coils is reduced. For a PM motor, changing the number of coils is further complicated by having to change the number of magnet poles, which may only be reduced by demagnetization.

- Mechanical RTFC, such as variable-gap control, can work for an axial-gap PM motor but is much more difficult for a radial-gap motor because of the requirement that the gap be precisely controlled by axial motion of a conical rotor. The usual problem remains of energy expended as the gap is opened and closed to accomplish field weakening.

\section{Inductance Control}

- The biggest contributor to the phase inductance in a fractional-slot motor is the slot inductance, which places a premium on the design of the slot-opening configuration. The slots are long and 
slender and are not necessarily the optimal design. This could pose problems in forming the laminations.

- The results of the losses in the magnet seem low.

- The higher fill factor of the concentrated winding allows a more compact motor, which leads to higher power density and higher specific power.

- For the 6-kW motor with soft magnetic material and a lower remanence requiring larger thicker magnets, values from the 2-dimensional Zhu method of calculating back-emf compare more favorably with experimental data than do values from the 1-dimensional method.

- The 6-kW motor analyzed with SPEED software was concurrently modeled with a per-phase equivalent circuit. The two analyses compared well with the experimental UWM results.

- The effect of stator slotting on permeance of the 6-kW motor has been calculated and compares well with UWM calculations.

- FreedomCAR specifies that the electric motor must be able to deliver $55 \mathrm{~kW}$ for 18 seconds. An additional desirable industrial requirement added to requests for proposals to motor vendors in FY 2005 was that the motor should deliver this intermittent power from 20 to $100 \%$ of maximum speed, which points out that an advanced cooling technique is a major requirement for this motor. To produce this amount of power, the required rms current is approximately $460 \mathrm{~A}$ and the rms bus voltage is approximately $224 \mathrm{~V}$, both of which exceed maximum specifications, which are $400 \mathrm{~A}$ and $200 \mathrm{~V}$. This design problem will be addressed in the next phase of this study.

- FreedomCAR specifies that the electric motor's efficiency should be greater than $93 \%$ from $10 \%$ to $100 \%$ of maximum speed. An additional desirable industrial requirement added to requests for proposals in FY 2005 is that this efficiency should be reached at $20 \%$ of rated torque. This will be difficult to attain and will demand accurate core loss calculations and place a premium on the type and thickness of the laminations.

\section{Inverter Control}

- Study regarding control of PMSMs

○ More inductance and more voltage are better.

$\circ$ Even with the benefit of both characteristic inductance and high voltage, CPA suffers from low efficiency.

- DMIC solves this problem by ensuring that the current used is proportional to load.

- Automobiles spend most of their time at half speed and partial load.

$\circ$ As gas prices soar and efficiency becomes more important to the consumer, DMIC has the potential to provide the highest efficiency possible.

- Study regarding validation of methodology

$\circ$ Expressions for the modulation index, $\mathrm{m}_{\mathrm{a}}$, and inverter lead angle, $\delta$, which were derived based on an algebraic fundamental frequency model, have been found to be very accurate when used in a detailed time domain simulation that includes PWM switching.

○ Expressions for average and rms currents in diodes, transistors, and thyristors were compared with detailed time domain simulations above and below base speed.

- Below base speed, where simulations for CPA and DMIC control are the same, simulated losses at carrier frequencies of 8505 and $2025 \mathrm{~Hz}$, agreed with theoretical model losses within $1 \%$.

- Above base speed, where separate simulations were necessary for CPA and DMIC, simulated losses for CPA control were within $2.5 \%$ of the theoretical model for transistor average and rms current and diode average currents. Simulated loss of the diode rms current, which was not quite as good, was within $8 \%$ of the theoretical model.

- Simulated losses for DMIC control were within $1 \%$ of the theoretical model for the average transistor, by-pass diode, and thyristor currents. Simulated loss of the transistor and thyristor rms currents, which were not quite as good, were within $9 \%$ of the theoretical model. 
- Since diode losses are generally 1/2 transistor losses, and conduction losses of the diodes are weighted more by forward voltage drop and average current than by diode resistance (rms current losses), the simplified theoretical model is sufficiently accurate for this study.

- Study regarding operation of PMSMs under CPA control

- Characteristic motor current, which permits operation at high CPSR, depends solely on machine parameters, $\mathrm{E}_{\mathrm{b}}, \Omega_{\mathrm{b}}$, and $\mathrm{L}$, and is independent of motor load and dc supply voltage. This lowers its efficiency at partial-load conditions. Providing voltage higher than necessary to support rated torque at base speed cannot reduce the current at high speed.

o It is advantageous to have the inductance higher than "optimal" because it enables the motor to develop the required power with lower current and attendant efficiency increase.

o Even more voltage than the minimum required for injecting rated current at base speed can be beneficial because of lower current and attendant efficiency increase.

$\circ$ The speed at which minimum current, $\mathrm{n}_{\min }$, occurs depends linearly on developed power and inversely on the maximum fundamental inverter voltage. $\mathrm{n}_{\min }$, is a unique speed, which varies with voltage, leaving room for optimization. If a motor spends a substantial amount of time at half speed, it could be desirable to choose a dc supply voltage that causes the minimum current at half speed. This would involve using a dc supply larger than the minimum and using control to restrain the torque envelope.

- Control of the voltage lead angle at high speeds allows a PMSM to operate at constant power, but it does not ensure operation within rated current. Inductance is the critical factor that ensures operation within rated current.

o The limiting current at high speed is mostly for field-weakening and therefore produces little torque.

○ At full load, the motor with characteristic inductance behaves as well with CPA control as with DMIC control.

- Study regarding operation of PMSMs under DMIC control

○ Higher inductance results in reduced current at only one speed and power level under CPA control, whereas DMIC control minimizes current at all power levels and speeds, $\mathrm{n}>2$.

- DMIC motor current is proportional to load at high speed, which leads to decreased current and attendant increased efficiency at partial load.

- Although originally for motors with low inductance, DMIC has potential for significant loss reduction for PMSMs with large inductances.

- DMIC control maintains an inverter power factor of 1 for all speeds greater than $\mathrm{n}_{\min }$, the speed at which minimum current occurs. Under CPA control $n_{\min }$ is a single speed at which a PMSM operates at an inverter power factor of 1 . At all speeds above $\mathrm{n}_{\min }$ the CPA power factor leads.

- With DMIC, high-speed current is inversely proportional to supply voltage so that extra supply voltage can lead to improved efficiency.

○ Depending on the application, particularly the speed/loss profile, loss reduction may more than compensate for the losses introduced by the addition of the SCRs required by DMIC.

$\circ$ At half speed and quarter load, Motor 1 under DMIC delivers 7.5\% (percentage points) more power at $3000 \mathrm{rpm}$ and $8.6 \%$ more power at $6000 \mathrm{rpm}$ than Motor 1 under CPA control.

- Study regarding the grade of SCR that may be used

o Increasing reverse recovery losses of thyristors, which increase with speed, is a problem that can be reduced with inverter-grade components at increased cost; however, cost and losses may be offset by efficiency enhancement.

- The question about converter-grade SCRs being able to sustain operation in the FreedomCAR application at a fundamental switching rate of $1.5 \mathrm{kHz}$ at top speed of $6000 \mathrm{rpm}$ must be answered in the laboratory by experimentation. 


\section{Reluctance Control}

- Preliminary analyses confirmed that

$\circ$ To reduce the magnitude of the characteristic current, which is the current required to completely cancel the PM magnetic flux, $I_{c h}=\frac{\lambda_{p m}}{L_{d}}$, one may reduce the magnitude of the PM flux linked or increase the value of the d-axis inductance.

o Since the saliency ratio is $\mathrm{L}_{\mathrm{q}} / \mathrm{L}_{\mathrm{d}}$, the equation for power delivered (using the convention that increasing $\mathrm{d}$-axis current increases power delivery) may be expressed as $P_{d e l}=I_{q}\left\lfloor E_{p m}+I_{d} L_{d} \omega_{e l}(\xi-1)\right]$, which shows that IPM motors with non-zero saliency are superior to surface-mounted motors for flux weakening because, under flux weakening conditions, they provide extra mechanical power output.

$\circ$ The equation $\delta \lambda_{p m}=-I_{d} L_{d} \delta \xi$ shows that increasing the saliency and d-axis inductance will reduce the PM flux linkage needed by a motor. This would enhance the motor's flux weakening and reduce the cost of PMs while maintaining the motor's power level.

$\circ$ The equation $\lambda_{p m}=k e^{-\frac{I_{d} \xi}{I_{c h}}}$ shows that for equal motor power output, the magnet strength needed diminishes exponentially with the saliency and with the amount of d-axis current relative to the characteristic current.

○ If one increases $L_{d}$ as recommended by the first and third bulleted items above, then one should increase $L_{q}$ at least proportionally to maintain saliency. Likewise, an increase in saliency ratio should be accomplished only by an increase in $L_{q}$.

- The role of reluctance in PM motors may be rephrased in terms of the work inductance together with the inverse of its preceding verb; i.e. "increase the inductance, $\mathrm{L}_{\mathrm{d}}$ " would be replaced by "decrease the reluctance, $R_{d}$."

- Preliminary studies of the three types of IPM motors showed

○ Torque at low speeds was significantly higher than for similar SPM motors, as expected based on earlier work.

○ The drop in power delivery with increasing speed is lower for all of the IPMs studied, as expected, because they have weaker magnet strength, which increases their maximum operating speed.

o For the PM motor with inset magnets, the optimal magnet fraction, which delivers $40 \mathrm{~kW}$ at a CPSR of 10 , is 0.69 .

- The use of lumped-parameter equations for modeling is appealing since it facilitates understanding; but it requires the determination of inductances, resistance, and flux linkages with the degree of detail appropriate for the simulation goals.

- Characterization of magnetic saturation is challenging since, especially in the case of IPM motors, magnetic flux paths vary significantly, not only with the rotor's position relative to the stator-generated rotating field but also with the magnitude of the stator's currents. It is best to have measured values of the lumped parameters. Next best is to obtain them by means of finite element computations. Most often, though, they are obtained by means of calculations based on geometry, equivalent circuits and adjustment factors.

- The focus of performance can be on power output or efficiency and may flip from one to the other depending on the application.

- It is necessary, in addition to the standard efficiency/torque maps, to determine the overall efficiency for a set of standard driving cycles. These results should be the criterion to define optimal configurations. 


\section{Future Direction}

\section{- Voltage Control}

- Use of the Z-source inverter with ICE-hybrid vehicles will require development of a bidirectional version to make use of regeneration.

- Flux Control

- To date, most of the efforts have focused on changing magnetic-flux-flow paths. We feel that the alternatives, which change the magnet properties or number of turns, should also be examined for applicability to FreedomCAR HEVs.

\section{- Inductance Control}

- Since slot inductance contributes most of the phase inductance, the design of the slot-opening configuration and its impact on forming laminations will be explored further in FY 2006.

- Calculation of core loss and magnet losses will be studied further, and a way to check their validity for fractional-slot PMSMs will be sought.

- Delivering $55 \mathrm{~kW}$ at $2000 \mathrm{rpm}$ with an integral slot PMSM requires an rms current of about 460 $\mathrm{A}$ and an rms bus voltage of about $224 \mathrm{~V}$, both of which exceed maximum specifications, which are $400 \mathrm{~A}$ and $200 \mathrm{~V}$. Similar difficulties are encountered for the fractional-slot PMSM. Designing around this problem will be addressed in the next phase of this study.

- Collaboration will continue with UWM to prepare a design package for fabrication of a 30/55-kW fractional-slot motor with concentrated windings.

\section{- Inverter Control}

- Laboratory experimentation will be necessary to determine if cheaper converter-grade SCRs are able to sustain operation in the FreedomCAR application at a fundamental switching rate of $1.5 \mathrm{kHz}$ at a top speed of $6000 \mathrm{rpm}$.

- Provide two control schemes for driving the 6-kW PMSM motor with fraction-slot concentrated windings.

- Scheme 1 will minimize current at high speeds to reduce operating costs at partial loads.

- Scheme 2 will be the least expensive.

- Rapid prototype the scheme or schemes with promise.

- Interface with a dynamometer and test to determine the partial load regions where operating costs may be reduced.

\section{- Reluctance Control}

- Technology developed in this task will be used to explore extending the CPSR of reluctance traction drive motors.

\section{Publications}

J. W. McKeever, S. C. Nelson, and G. J. Su, Boost Converters for Gas Electric and Fuel Cell Hybrid Electric Vehicles, ORNL/TM-2005/531, Oak Ridge National Laboratory, May 2005.

P. J. Otaduy and W. C. Johnson, The Role of Reluctance in PM Motors, ORNL/TM-2005/86, Oak Ridge National Laboratory, April 2005.

P. J. Otaduy and J. W. McKeever, Real Time Flux Control in PM Motors, ORNL/TM-2005/175, Oak Ridge National Laboratory, Sept. 2005.

J. M. Bailey and J. W. McKeever, Fractional-Slot Surface Mounted PM Motors with Concentrated Windings for HEV Traction Drives, ORNL/TM-2005/183, Oak Ridge National Laboratory, Sept., 2005.

J. S. Lawler and J. W. Mckeever, Control of Surface Mounted Permanent Magnet Motors with Special Application to Fractional-slot Motors with Concentrated Windings, ORNL/TM-2005/184, Oak Ridge National Laboratory, Sept. 2005.

P. J. Otaduy and J. W. McKeever, Modeling Reluctance Assisted PM Moators, ORNL/TM-1005/185, Oak Ridge National Laboratory, Oct. 2005. 
J. S. Lawler, J. M. Bailey, and J. W. McKeever, "Minimum Current Magnitude Control of Surface PM Synchronous Machines during Constant Power Operation," IEEE Power Electronics Letters, 3(2), June 2005.

J. S. Lawler, J. M. Bailey, J. W. McKeever, and P. J. Otaduy, "Impact of Continuous Conduction on the Constant Power Speed Range of the Switched Reluctance Motor," Int. Electric Machines and Drives Conf., San Antonio, TX, May 15-18, 2005.

\section{References}

1. M. Okamura, E. Sato, and S. Sasake, "Development of Hybrid Electric Drive System Using a Boost Converter," EVS-20, Session 5, The 20th International Electric Vehicle Symposium and Exposition, Long Beach, CA, Nov. 15-19, 2003.

2. F. Z. Peng, "Z-source Inverter," Proc. Of IEEE, IAS 2002, pp. 775-781.

3. O. I. Elgerd, Electrical Energy Systems Theory: Introduction, McGraw-Hill Book Company, chapter 4, 1971.

4. J. S. Hsu, "Direct Control of Air Gap Flux in Permanent Magnet Machines," Paper No. TR7-001 in IEEE Transactions on Energy Conversions, 2000.

5. J. S. Hsu, "High Strength Undiffused Brushless Electric Motors and Generators," Patent 6,057,622, June 3, 2003.

6. V. Ostovic, "Pole-Changing Permanent Magnet Machines," IEEE Trans. Ind. App. 38(6), November/December 2002.

7. R. J. Parker, Advances in Permanent Magnetism, a Wiley-Interscience publication, John Wiley and Sons, 1990.

8. G. Liang, J. Miller, and X. Xu, "A Vehicle Electric Power Generation System with Improved Output Power and Efficiency," IEEE Trans. Ind. App., 35(6), November/December, 1999.

9. S. C. Oh and A. Emadi, "Test and Simulation of Axial Flux Motor Characteristics for Hybrid Electric Vehicles," IEEE Trans. On Vehicular Technology, 53(3) May, 2004.

10. T. M. Jahns, G. B. Klieman, and T. W. Newmann, "Interior Permanent Magnet Synchronous Motors for Adjustable-Speed Drives," IEEE Trans. Ind. App., IA-22(4), 738-747, July/August, 1986.

11. T. M. Jahns, Interim Report:Cost Reduction Opportunities for Permanent Magnet Synchronous Machines, Oak Ridge National Laboratory, May 24, 2004.

12. A. M. El-Refaie and T. M. Jahns, "Optimal Flux Weakening in Surface PM Machines using Concentrated Windings," 2004 IEEE Ind. App. Soc. Annual Meetings, Seattle, WA, October 2004 (scheduled for publication in the IEEE Trans. Ind. App., May/June 2005).

13. A. M. El-Refaie, T.M. Jahns, P.J. McCleer, and J. W. McKeever, "Experimental Verification of Optimal Flux Weakening in Surface PM Machines Using Concentrated Windings," Conf. Rec. IEEE Ind. App. Soc. Annual Meeting, Hong Kong, China, October 2-6, 2005.

14. J. S. Lawler, J. M. Bailey, and J. W. McKeever, "Minimum Current Magnitude Control of Surface PM Synchronous Machines During Constant Power Operation," IEEE Power Electronics Letters, 1(2), June 2005.

15. T. Sebastian and G. R. Slemons, "Operating Limit of Inverter-Driven Permanent Magnet Motor Drives," Trans. Ind. App., IA-23(2), 327-333, March 1987.

16. J. S. Lawler, J. M. Bailey, and J. W. McKeever, Extended Constant Power Speed Range of the Brushless Dc Motor through Dual Mode Inverter Control, ORNL/TM-2000/130, Oak Ridge National Laboratory, 2000.

17. N. Mohan, T. Undeland, and W. Robbins, Power Electronics: Converters, Applications, and Design, Chapter 5, John Wiley and Sons, 1989.

18. F. Z. Peng, "Z-Source Inverter," IEEE Transactions of Industry Applications, 39(2), March/April 2003.

19. F. Z. Peng et al., "Z-Source Inverter for Adjustable Speed Drives," IEEE Power Electronics Letters, 1(2), 33-35June 2003. 
20. M. Okamura, E. Sato, and S. Sasaki, "Development of Hybrid Electric Drive System Using a Boost Converter," EVS-20, Session 5, The 20th International Electric Vehicle Symposium and Exposition, Long Beach, California, November 15-19, 2003.

21. B. Ozpineci et al., "Optimum Fuel Cell Utilization with Multilevel DC-DC Converters," pp. 15721576 in Conference Proceedings of the 19th Annual IEEE Applied Power Electronics Conference and Exposition, 3, 2004.

22. Michigan State University, "Z-Source Inverter for Fuel Cell Vehicles," submitted to Oak Ridge National Laboratory's Engineering Science and Technology Division at the Power Electronics and Electric Machines Research Center, Oak Ridge TN, August 31, 2005.

23. Fang Z. Peng, slide 13 of presentation "Z-Source Inverter for Hybrid Electric and Fuel Cell Powered Vehicles," DOE FreedomCAR and Vehicle Technologies Program Annual Review of Advanced Power Electronics and Electrical Machines, June 7-9, 2004.

24. D. W. Novotny and T. A. Lippo, Vector Control and Dynamics of AC Drives, Clarendon Press, Oxford, 1996 (republished 2000).

25. B. Sneyers, D. W. Novotny, and T. A. Lipo, "Field-Weakening in Buried Permanent Magnet AC Motors Drives," IEEE Transactions, IA-21, 398-407, 1985.

26. R. F. Schiferl and T. A. Lipo, "Power Capability of Salient Pole Permanent Magnet Synchronous Motor in Variable Speed Drive Applications," IEE Transactions, IA-26, 115-123, 1990.

27. W. L. Soong and T. E. J. Miller, "Field Weakening Performance of Brushless Synchronous AC Motor Drives," pp. 331-340 in IEE Proceedings of the Electronics Power Appl., 141(6), November 1994.

28. A. K. Adnanes and T. M. Undeland, "Optimum Torque Performance in PMSM Drives Above Rated Speed," pp. 169-175 in Proceedings of IEEE Industry Applications Society Annual Meeting, October 1991.

29. Liwschitz, Garik, and Whipple, Electric Machinery Vol II AC Machines, D. Von Nostrand Company, New York, 1946.

30. M. M. Liwschitz, "Distribution Factors and Pitch Factors of the Harmonics of Fractional-Slot Windings," AIEE Transactions 62, 664-666, October 1943.

31. J. Cros and P. Viarouge, "Synthesis of High Performance PM Motors with Concentrated Windings," IEEE Transactions on Energy Conversion, 17(2), June 2002.

32. F. Magnussen and C. Sadarangani, "Winding Factors and Joule Losses of Permanent Magnet Machines with Concentrated Windings," pp. 333-339 in Proceedings of 2003 IEEE International Electric Machines \& Drives Conference (IEMDC), 1, Madison, Wisconsin, June 2003.

33. Z. Q. Zhu, "Instantaneous Magnetic Field Distribution in Brushless Permanent Magnet dc Motors, Part I: Open-Circuit Field," IEEE Transactions on Magnetics, 29(1), January 1993.

34. A. K. Adnanes, "Torque Analysis of Permanent Magnet Synchronous Motors," pp. 695-701 in 23rd Annual IEEE Power Electronics Specialists Conference Record, June 29-July 3, 1992.

35. A. M. El-Refaie and T. M. Jahns, "Optimal Flux Weakening in Surface PM Machines using Fractional-Slot Concentrated Windings," IEEE Trans. Industry Applications, 41(3), 790-800, MayJune, 2005.

36. A. E. Fitzgerald, C. Kingsley, Jr., and S. D. Umans, Electric Machinery, McGraw-Hill, 1990.

37. J. S. Lai, "Fuel Cell Power Conversion Technologies," Presentation to ORNL Power Electronics and Electric Machines Research Center, September 2, 2005. 


\subsection{Electric Motor Research and Development}

Principal Investigator: John S. Hsu

Team Members: Curtis Ayers, Steven Campbell, Chester Coomer, Michael Jenkins, Kirk Lowe, Randy Wiles

Oak Ridge National Laboratory

National Transportation Research Center

2360 Cherahala Boulevard

Knoxville, TN 37932

Voice: 865-946-1325; Fax: 865-946-1262; E-mail: hsujs@ornl.gov

DOE Technology Development Manager: Susan A. Rogers

Voice: 202-586-8997; Fax: 202-586-1600; E-mail: Susan.Rogers@ee.doe.gov

ORNL Program Manager: Mitch Olszewski

Voice: 865-946-1350; Fax: 865-946-1262; E-mail: olszewskim@ornl.gov

\section{Objectives}

The drive motor is an extremely important component in both fuel-cell-powered and hybrid electric vehicles (HEVs). It provides all or part of the torque for moving the vehicle. All other components in the drive train are dependent upon the traction motor for providing the torque in the most efficient manner that meets system cost, size, weight, and reliability targets. Investigations of a reluctance interior permanent magnet (RIPM) with brushless field excitation (RIPM-BFE) machine, of a hybrid secondary uncluttered PM (HSUPM) machine, and of corona starting voltages with a conventional insulation system were all part of the motor development work conducted in FY 2005.

The objective of the FY 2005 research and development effort was to build an RIPM-BFE machine having a core length $30 \%$ shorter than that of the Toyota Prius motor (with the same diameter and same speed). In FY 2005, a HSUPM machine was conceptually designed. It reduces costs by combining the generator and the motor into one machine so that only one set of PMs and one frame are required. Torque production can be increased as a result of the rotating armature.

The objective of the corona/voltage study was to see how much the voltage could be increased in a motor before corona occurs in order to assess the motor's insulation capability. This gives valuable background data for considering high-voltage drive systems.

\section{Approach}

The project scope includes the design, simulation, drawings, fabrication, and testing of the RIPMBFE machine, as well as the conceptual design of an HSUPM machine, in an effort to reduce motor size. Corona/motor voltage investigations were also performed to ascertain the voltage limitations and penalties associated with the higher-voltage operation of motors.

\section{Major Accomplishments}

The detailed results of the development of the RIPM-BFE motor are presented in the reports Interior Permanent Magnet Reluctance Machine with Brushless Field Excitation, ORNL/TM-2005/222, September 2005, and Finite-Element Analyses of An IPM Reluctance Motor with Rotor Side-Poles, ORNL/TM-2005/209, September 2005. Overviews of these reports will be provided later. In short, the concept of the RIPM-BFE machine was proved viable. The BFE coils were proved to be able to enhance and weaken the air-gap flux. This research led to a new concept for a machine with side PMs without 
excitation coils. Engineering designs will be needed to optimize the design of this machine in FY 2006. The simulation software also needs to be improved to reflect the detail of the engineering drawings.

Preliminary conceptual rotor drawings of the HSUPM machine were generated in FY 2005. The proof of concept work will continue with the fabrication, design, and testing in FY 2006.

There is a strong desire on the part of the auto industry to push the motor voltage higher to reduce both the inverter size and the starting battery current. The corona tests performed in FY 2005 provide information for permissible starting voltages for the insulation systems of $480-\mathrm{V}$ class motors. The detailed results are given in Corona and Motor Voltage Interim Report, ORNL/TM-2005/60, April 2005. The results from this report show that motor insulation requirements dictate the voltage limits for a highvoltage system.

\section{$\underline{\text { Technical Discussion }}$}

\section{RIPM-BFE Motor}

In a conventional PM machine, the air-gap flux produced by the PM is fixed. It is difficult to enhance the air-gap flux density because of limitations of the PMs in a series-magnetic circuit. However, the airgap flux density can be weakened by using power electronic field weakening to the limit of demagnetization of the PMs. This report presents the test results of controlling the PM air-gap flux density through the use of a stationary brushless excitation coil in an RIPM-BFE motor. Through the use of this technology, the air-gap flux density can be either enhanced or weakened. There is no concern with demagnetizing the PMs during field weakening. The leakage flux of the excitation coil through the PMs is blocked. The prototype motor built on this principle confirms the concept of flux enhancement and weakening through the use of excitation coils.

The RIPM-BFE was invented at Oak Ridge National Laboratory (ORNL). It offers a high torque per ampere by using a brushless excitation coil to enhance the flux. The motor eliminates the system need for a dc/dc boost converter at medium and high speeds. The core loss of the motor is low because the flux can be weakened through the excitation coil.

The design of the sample RIPM-BFE prototype machine is shown in Figure 1.

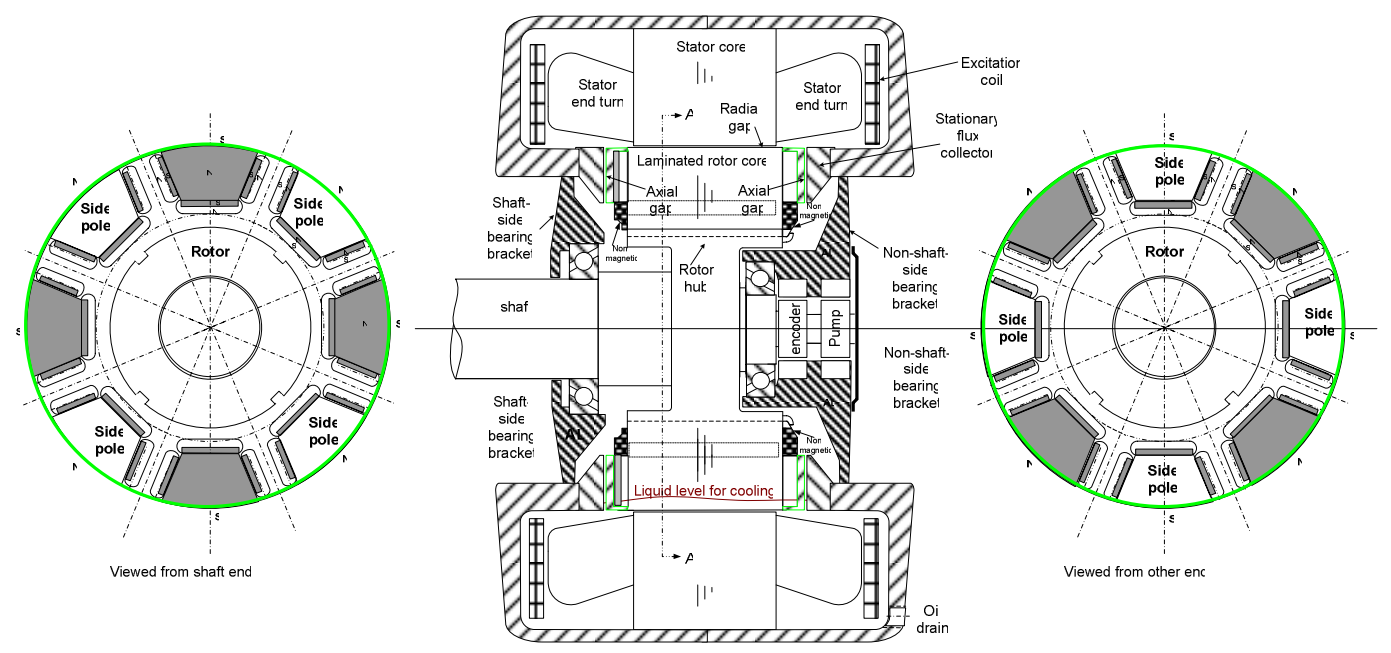

Figure 1. A sample RIPM-BFE prototype machine.

The electro-mechanical simulation was completed on the RIPM-BFE machine. It showed positive results for the overall design characteristics as well as the ability to weaken/enhance the magnetic flux of the machine. The simulations were performed, with a magnet thickness of $0.240 \mathrm{in}$. Engineering drawings 
were completed and sent to both ORNL and outside fabrication facilities for building the prototype machine.

Magnets for the RIPM-BFE rotor were fabricated by vendors outside of ORNL. The rotor laminations were designed to closely match the electro-mechanical simulated lamination design as seen in Figure 2. These laminations were fabricated from 0.014-in.-thick coated silicon steel (29 Ga. M19 C5 non-oriented fully processed). For comparison purposes, the laminations were fabricated with a diameter identical to that of the 2005 Toyota Prius hybrid system (THS II) drive system motor. The rotor inner diameter was modified from that of the Prius in order to incorporate the magnets.

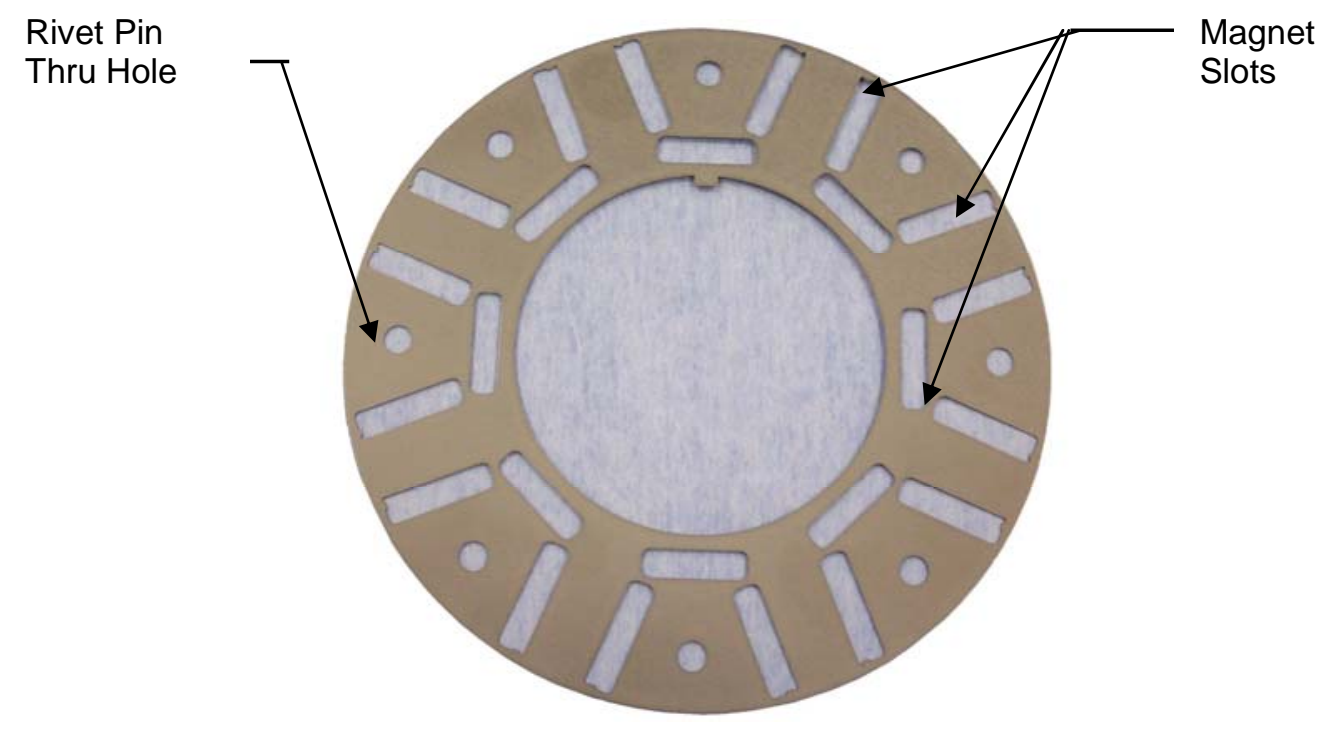

Figure 2. RIPM-BFE rotor lamination.

The stator used in the RIPM-BFE machine is a thinner version of the THS II traction drive motor (see Figure 3). The stator lamination stack was reduced to a final thickness of $2.5 \mathrm{in}$. The stator was re-wound and inductance and resistance measurements were recorded between each phase of the windings to ensure an electrically balanced stator.

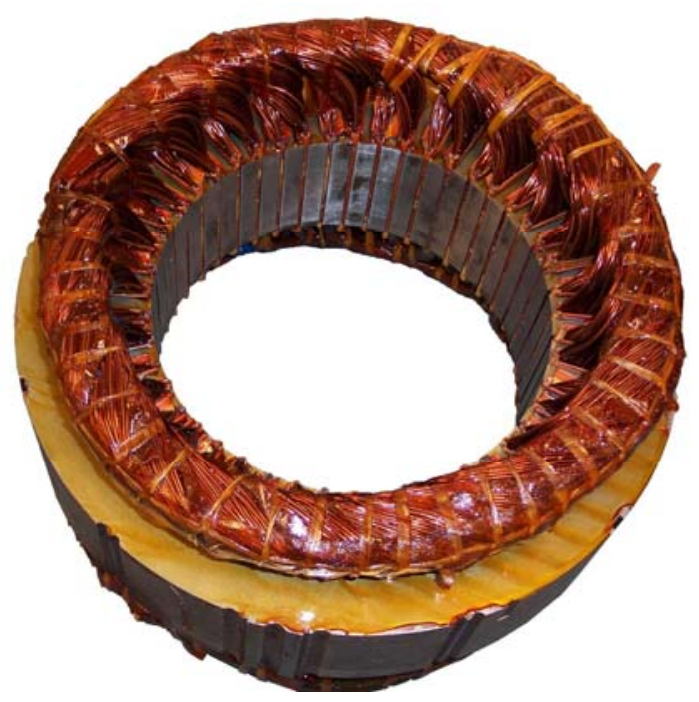

Figure 3. RIPM-BFE stator. 
The excitation coils were fabricated at ORNL. Each of the two coils was fabricated from 20-gauge class " $H$ " magnet wire and each contained 880 turns. To check the electrical balance of each coil, a resistive measurement was taken. The coils had a resistance of 19.30 and 19.37 ohms, respectively. Figure 4 shows the finished excitation coils.
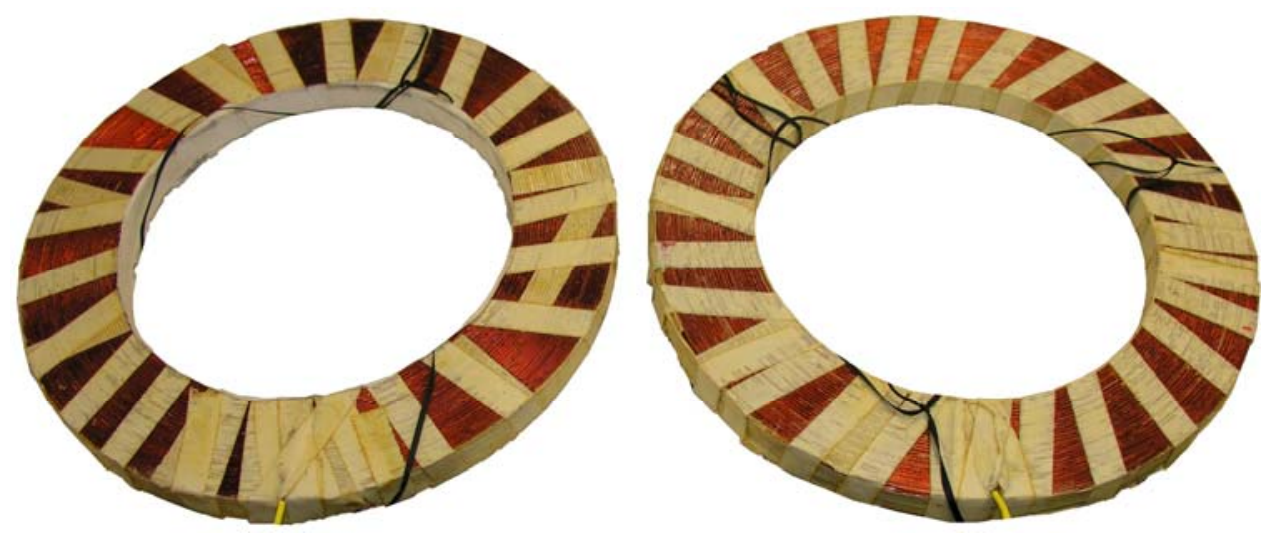

Figure 4. RIPM-BFE motor excitation coil.

The rotor laminations were placed over the rotor hub and mechanically fastened to the hub using the aluminum "spider" shown in Figure 5. Non-permanent magnetic pole pieces were placed around each face of the rotor in the spider slots, as shown in Figure 5. Additionally, the laminations were pulled together using the non-permanent magnet pole piece and a rivet-pin that passes through the laminations and is fastened into position with screws and the non-permanent magnetic pole piece. The 0.100 -in. pole magnets were placed at the locations shown in Figure 5 and then held in position with the magnet retaining ring (see Figure 6).

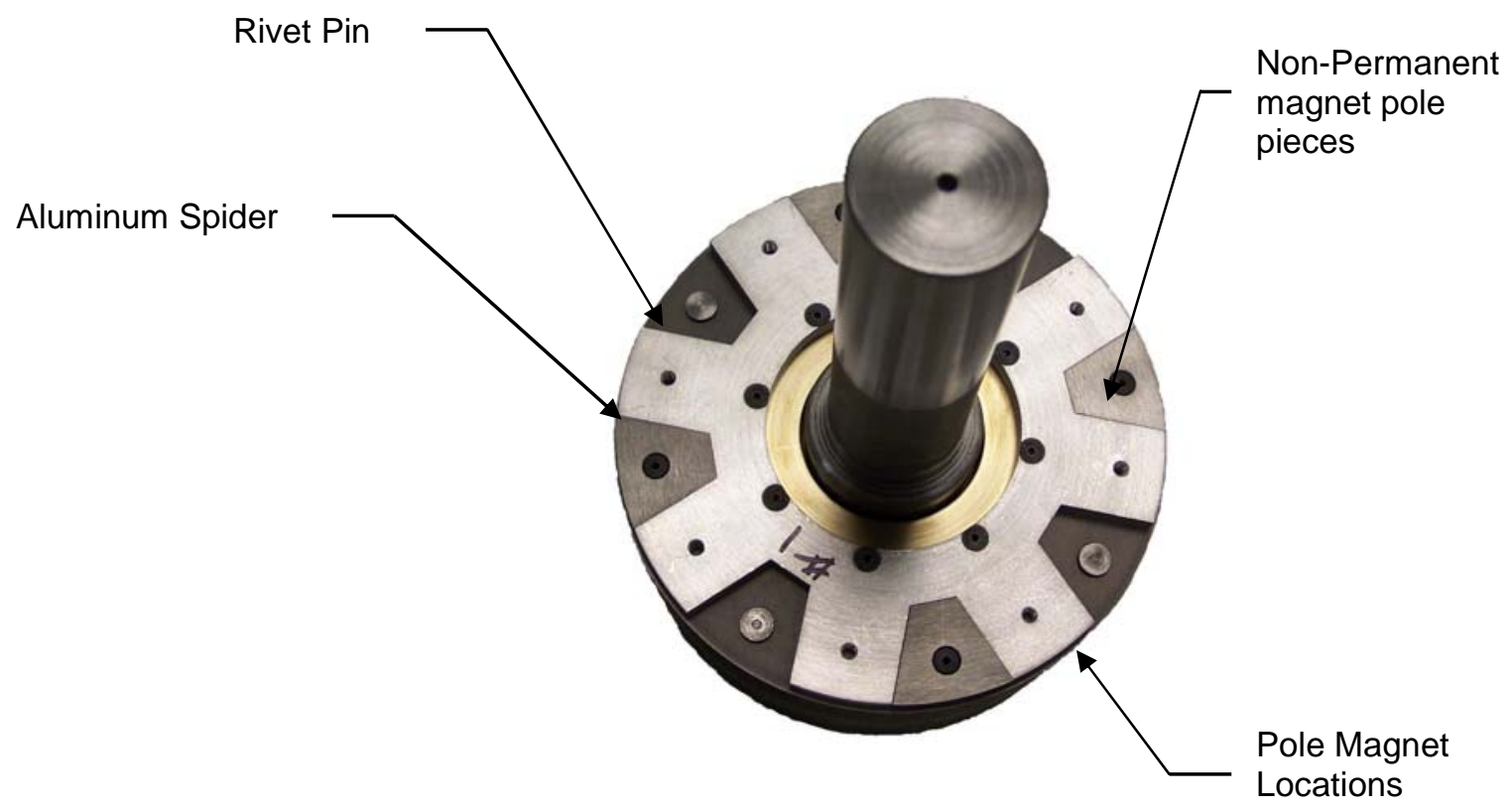

Figure 5. RIPM-BFE rotor. 


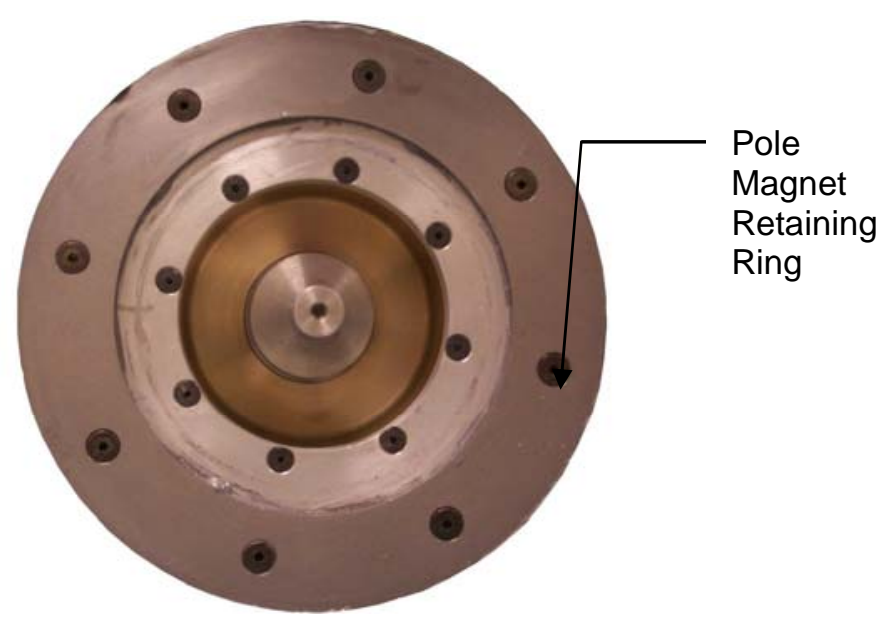

Figure 6. Assembled RIPM-BFE rotor.

The 0.100-in. magnets were placed axially in the lamination slots, and all side pole and axial lamination magnets were fixed in place with Master Bond Supreme 10HT epoxy. The epoxy was baked at $100^{\circ} \mathrm{C}$ for a minimum of 4 hours to facilitate the curing process, but not at a high enough temperature to permanently de-magnetize the PMs. Once the rotor was assembled, it was returned to the fabrication shop and each face of the rotor was trued. Non-magnetic material was machined and used as a fastening ring and heat-shrunk around the circumference of each of the rotor faces. This ring was used to prevent the pole magnets from being slung out in the event that the epoxy failed during operation. Mechanical measurements were made at each of the eight mechanical fastening positions and recorded. The mean thickness of the assembled rotor was 3.515 in.

The motor housings were assembled with spray rings mounted along the inside diameter at the top edge of both the excitation coil and the stator windings (see Figure 7). These spray rings will enable cooling oil to be pumped through the motor and sprayed directly onto both the excitation coil and the stator windings. The cooling oil will then be gravity-fed to the bottom of the motor and pumped through a heat exchanger.

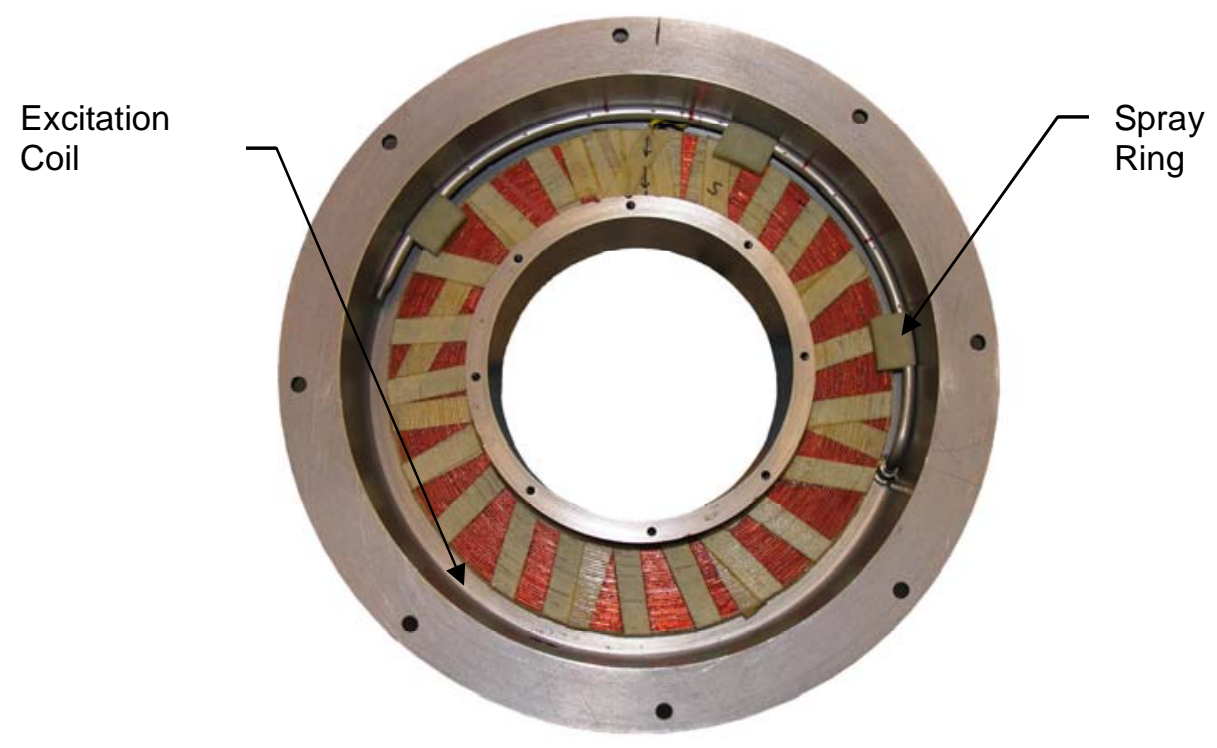

Figure 7. RIPM-BFE motor housing with excitation coil and spray ring. 
After the stator and rotor were inserted into the motor housing, the radial gap between the two was measured. A total of 0.028 in. of radial gap was present. Gauss measurements were taken to record the radial air-gap magnetic flux at each stator tooth. These measurements revealed that the rotor magnetism was uniform and all magnets were in their proper orientation. The axial air-gap was measured between the magnetic retaining ring on the rotor face and the motor housing. Each side of the rotor had an air gap of 0.067 in.

The following figures show the comparison of the air-gap flux densities between the ORNL 0.240-in. thick magnet motor without field excitation and the THS II motor. The maximum air-gap flux density of the ORNL motor is 1.27 Tesla, which is 1.63 times the 0.78 -Tesla flux density of the THS II. Figures 8 and 9 represent the air-gap flux density of the ORNL RIPM-BFE motor and of the THS II motor, respectively.

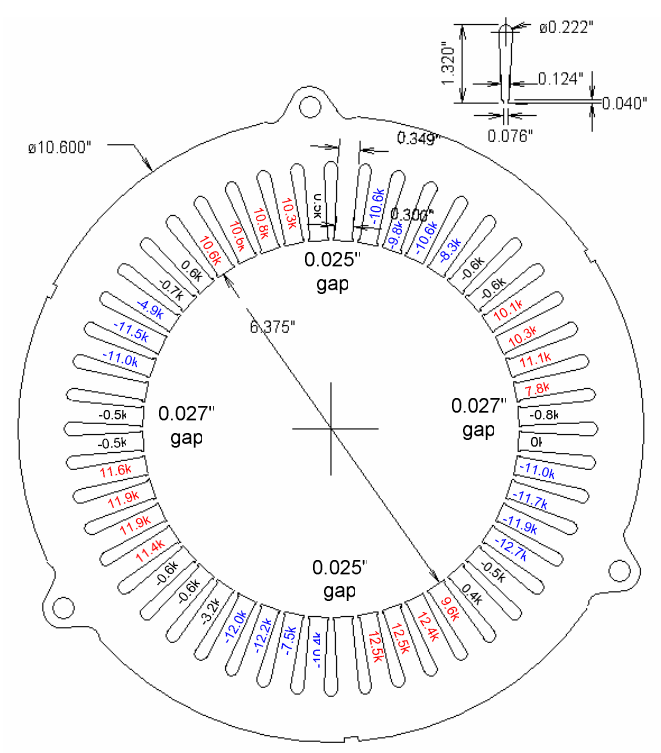

Figure 8. Air-gap flux density distribution of RIPM-BFE 2.5-in. core-length motor.

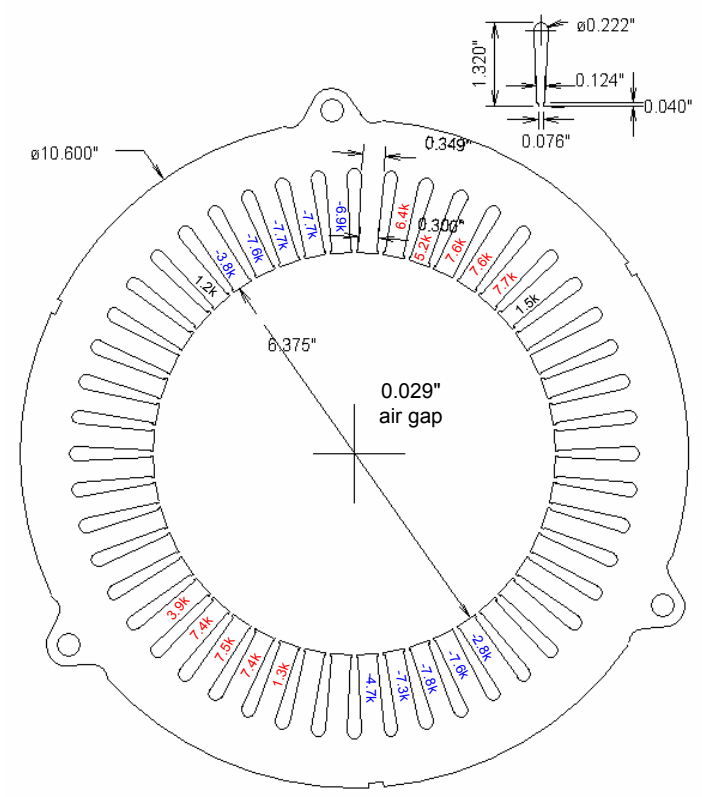

Figure 9. Air-gap flux density distribution of THS II 3.3-in. core-length motor. 
As an alternative approach, the motor can be simplified to employ side PMs to produce a strong fixed PM machine without the use of field excitation.

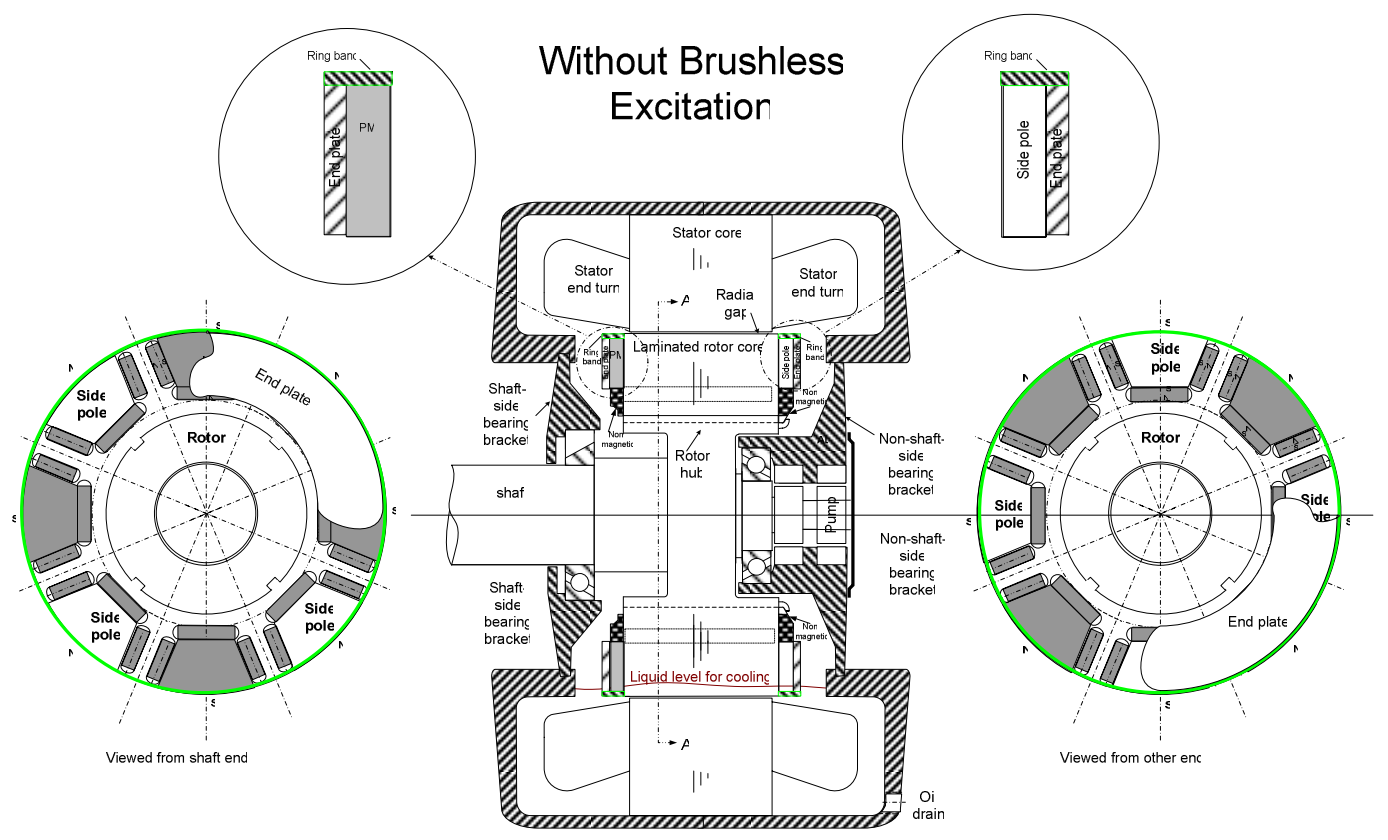

Figure 10. Machine design with side PMs to produce a strong fixed PM machine without field excitation.

Figures 11 and 12 show the locked-rotor torque versus rotor angular position at various field excitations. The tests were conducted at 50, 100, 150, 200, and $250 \mathrm{~A}$.

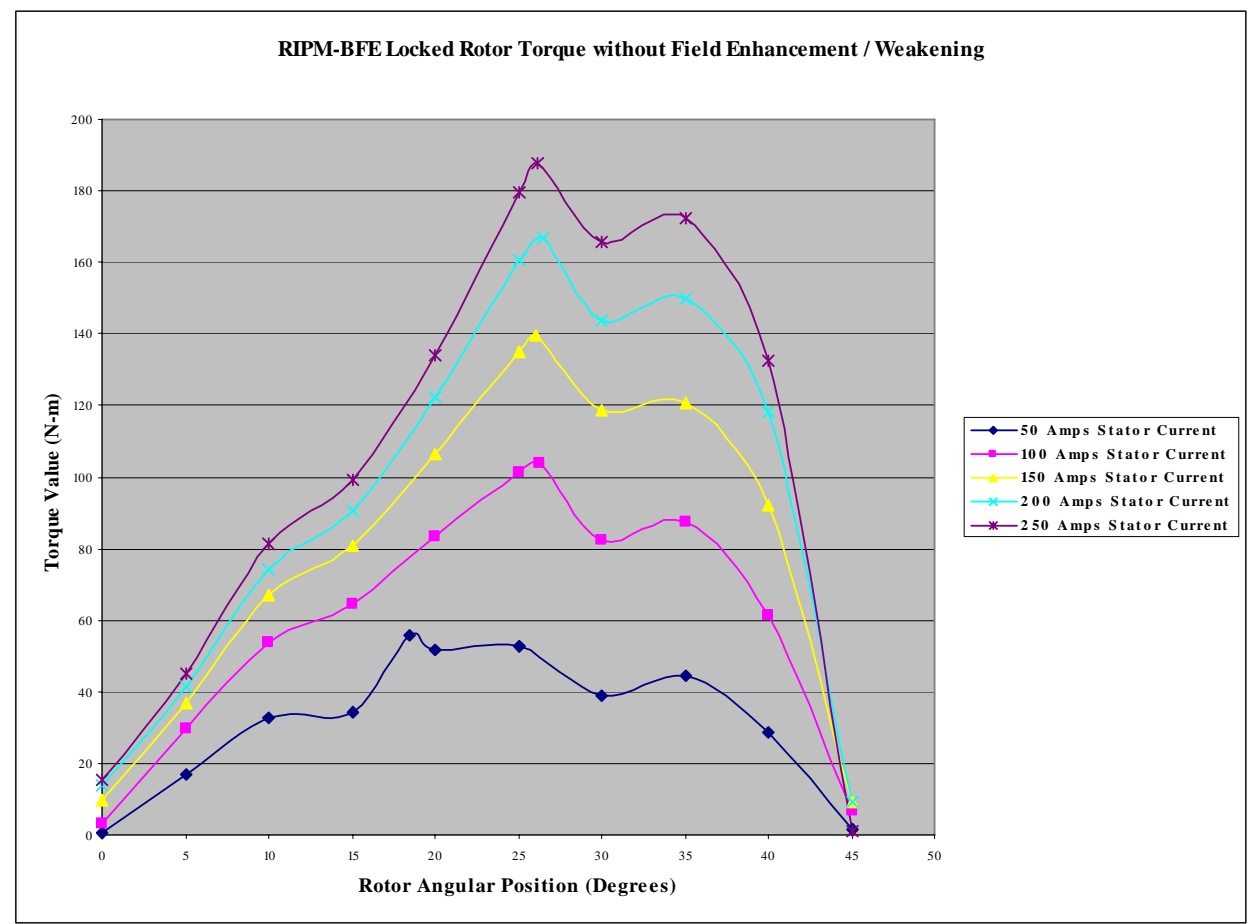

Figure 11. RIPM-BFE locked-rotor torque vs rotor angular position without field enhancement/weakening. 


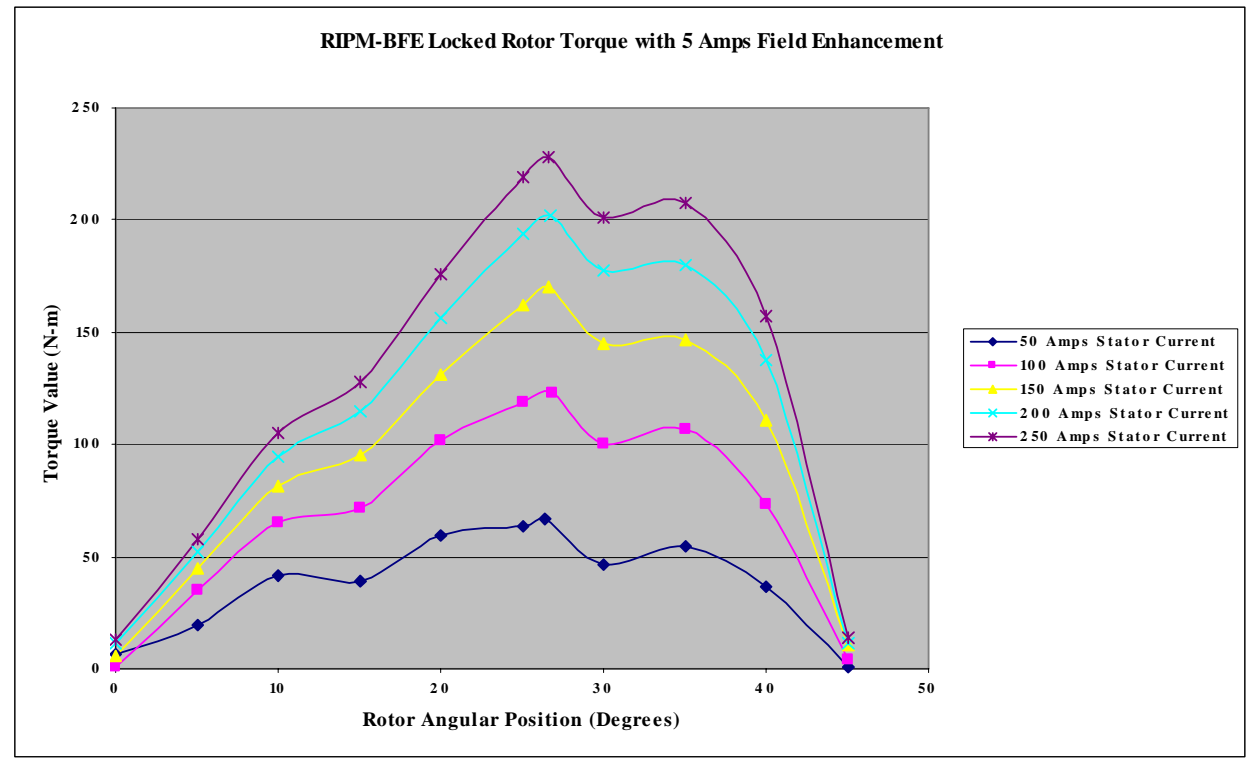

Figure 12. RIPM-BFE locked-rotor torque vs rotor angular position with $5 \mathrm{~A}$ of field enhancement.

Figure 13 shows locked-rotor torque comparisons between the 3.3-in.core length Prius motor and the 2.5-in. RIPM-BFE motor obtained through testing. The RIPM-BFE motor produces a stronger PM torque even with a shorter core length, but it has a weaker reluctance torque component, especially at higher currents. This indicates that magnetic saturation may be occurring. Further engineering work can solve this problem through optimization of the design.

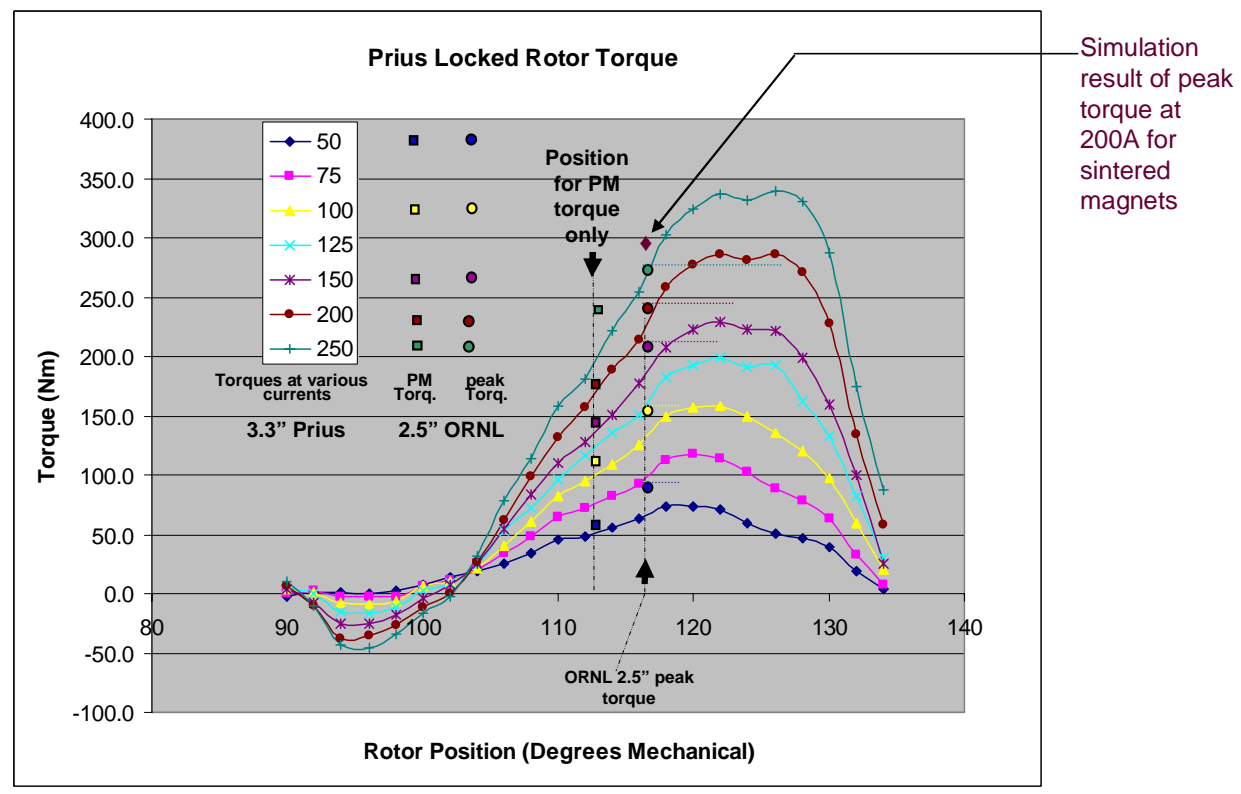

Figure 13. Comparison of PM torque components and peak-torque components between a 2.5-in. ORNL machine and a 3.3-in. THSII machine. 


\section{Corona Initiating Voltage for 480-V (rms) Insulation System}

Electric power is equal to the product of voltage and current. For a given power, if a higher voltage and lower current are used, the size of the cable and the inverter switching components necessary to carry the current can be reduced. The use of higher voltage also requires higher-quality and thicker electrical insulation that reduces the available slot area for motor windings. One mechanism of insulation breakdown is corona that gradually erodes the insulation and shortens the life expectancy of motors.

Figure 14 shows the corona test setup. A neon transformer with $120-15,000 \mathrm{~V}$ per side with the center tap to ground was used to produce high voltages. Smooth sinusoidal utility power was supplied to the primary winding of the neon transformer through a $120-\mathrm{V}$ isolation transformer. An inductor coil wound on a ferrite core was used to sense the corona discharging current. The two terminals of the inductor coil were connected to ground and to the center tap of the high-voltage winding of the neon transformer. The oscilloscope input probes were connected across the inductor to pick up the corona discharging current signals. A current limiter was connected in series with the high-voltage output to protect the high-voltage winding from over-current from the neon transformer. Silicone gel, silicone oil, or high-voltage putty was used to cover all exposed conductors at the non-grounded, high-voltage side to prevent corona produced by the instrumentation setup. After the power to the setup was switched off, an insulated grounding pole was used to discharge any energy stored in the setup before its reconnection. High-voltage gloves and insulation pads were used for protection during the tests.

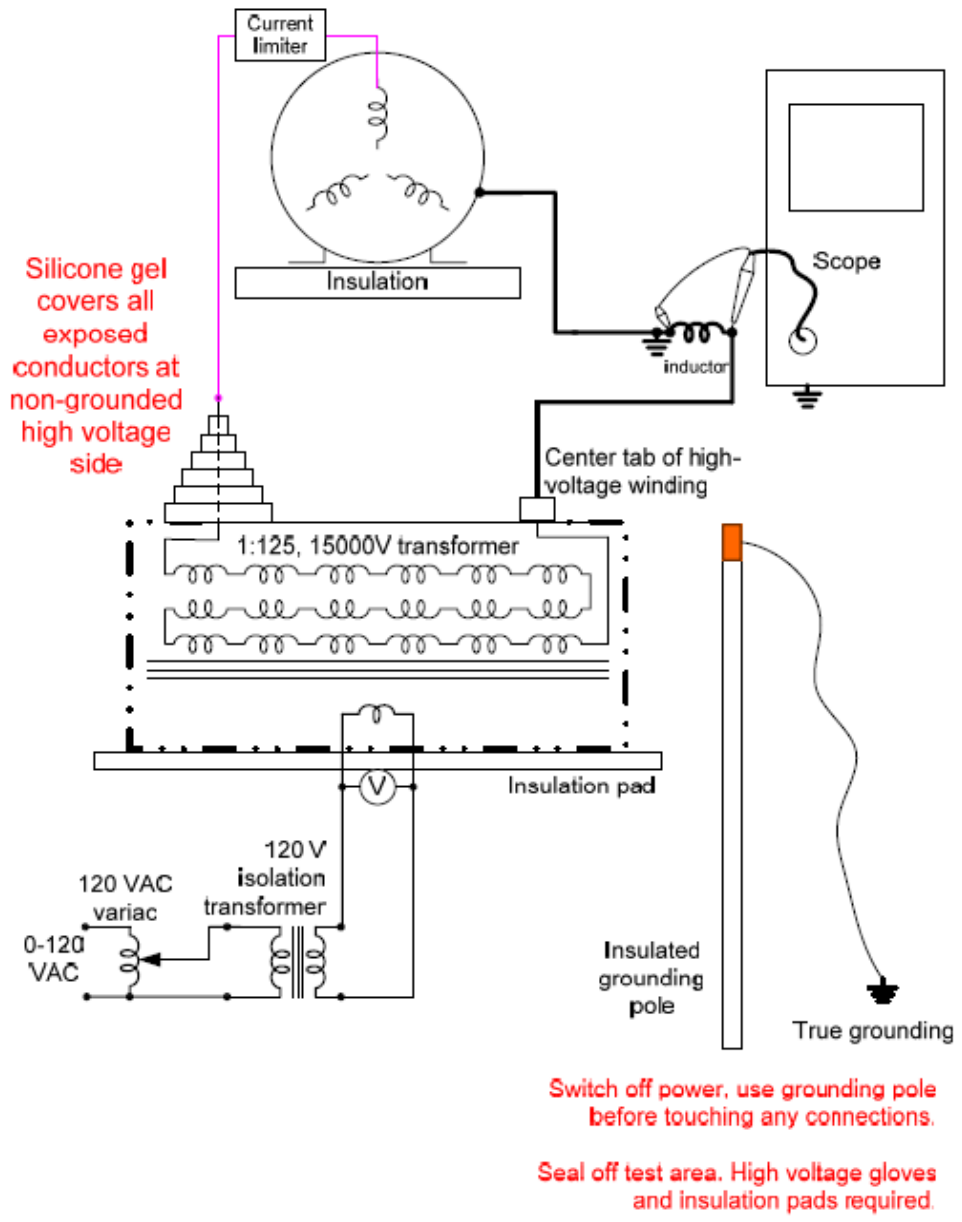

Figure 14. Corona initiating voltage test setup. 
To verify that the test setup did not produce a false corona signal before the high-voltage lead was connected to the motor, the high-voltage lead end was covered with high-voltage putty. A clean trace (Figure 15) was observed while the high voltage was adjusted through the range of 0 to 9000 Vrms.

For motors used in electric vehicles and HEVs, the back-emf at high speeds can be higher than the inverter output voltage, causing corona. High-voltage spikes due to inverter switching can also be a source of corona. To determine how high the voltage can be raised for electric vehicle and HEV applications, this investigation establishes the voltage where corona initiates in a National Electrical Manufacturers Association mush-wound stator and an HEV bobbin-wound stator. Table 1 summarizes the test results at $60 \mathrm{~Hz}$. The phase-to-phase corona initiates at only $1638 \mathrm{Vrms}$ for a mush-wound stator (Figure 16). The phase-to-ground (or frame) corona initiates higher at $2650 \mathrm{Vrms}$. The bobbin-wound

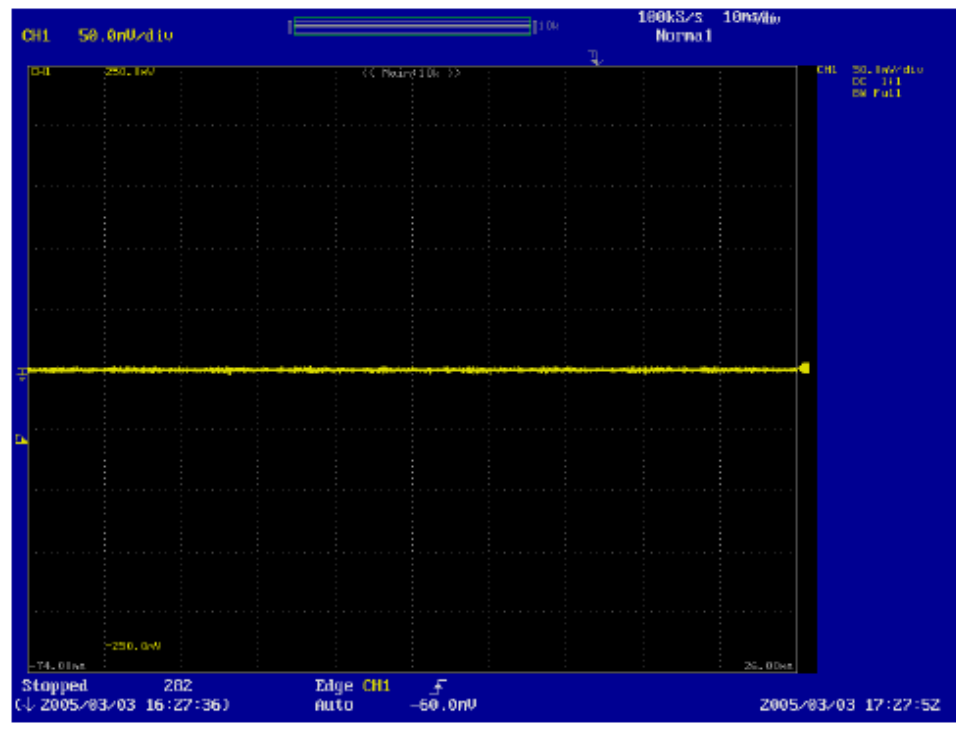

Figure 15. Clean trace without corona from 0 to $9000 \mathrm{Vrms}$ with silicone-sealed lead end.

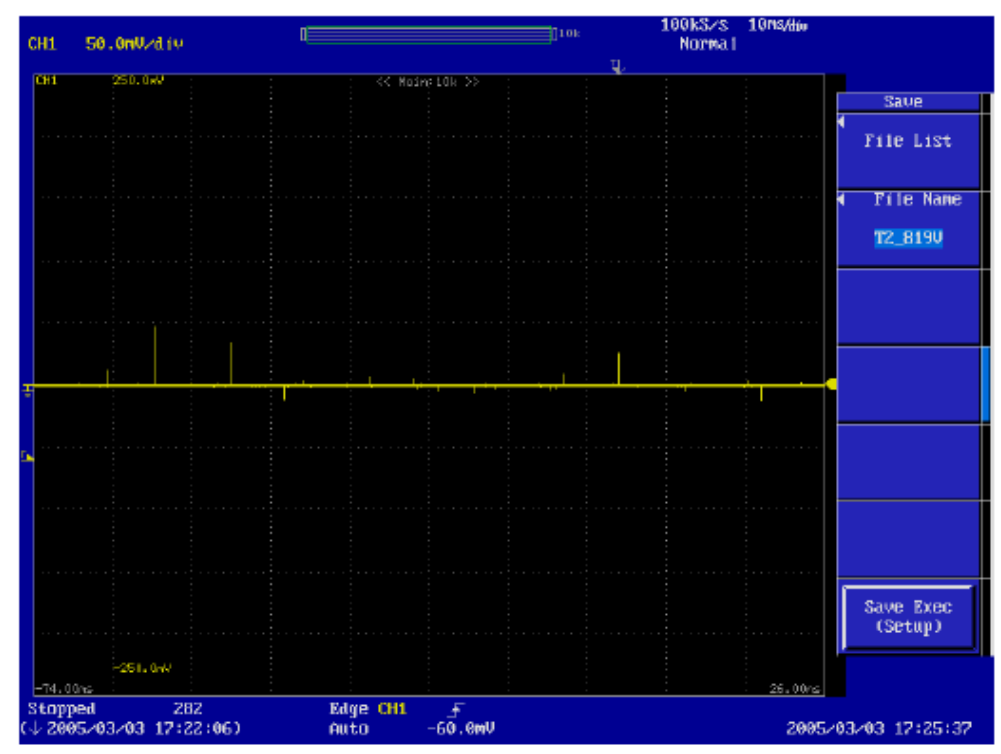

Figure 16. Corona initiating at $\mathbf{1 6 3 8}$ Vrms for phase-to-phase and phase-to-ground test. 
Table 1. Summary of test results of corona

\begin{tabular}{|c|l|c|}
\hline Winding type & & Corona initiating voltage (Vrms) \\
\hline Mush-wound stator & Phase-to-frame & 2650 \\
\cline { 2 - 3 } & Phase-to-phase & 1638 \\
\hline Bobbin-wound stator & Phase-to-frame & 3176 \\
\hline
\end{tabular}

stator shows slightly better performance than the mush-wound stator with a higher corona-initiating voltage of 3176 Vrms.

It should be pointed out that this corona-initiating voltage is for clean sinusoidal waveforms only. For inverter-fed motors, there are additional voltage spikes due to phase width modulation switching, The voltage where corona initiates would need to be reduced, depending on the magnitude of the spikes. The insulation system of mush-wound winding motors must be carefully examined because its phase-to-phase corona- initiating voltage is low.

\section{Conclusion}

The RIPM-BFE motor concept has been proved. Engineering modification of the design is required to optimize the motor performance. The RIPM-BFE motor was simulated and built with a stator core length of $2.5 \mathrm{in} ., 0.8$ in shorter than the 3.3 in. of the THS II stator core. Its back-emf is higher than that of the THS II motor, which is indicative of the enhancement of the air-gap flux density in the ORNL motor. The ORNL PM torque component is slightly higher than that of the THS II motor at all stator current levels. The reluctance torque component of the RIPM-BFE motor is about the same at a low stator current of 50 A. When the stator current increases to $250 \mathrm{~A}$, there is a significant difference between the ORNL motor and the THS II motor reluctance torque components. The RIPM-BFE motor requires further design modification to deal with this saturation issue to achieve a higher-reluctance torque component at highphase currents.

The RIPM-BFE motor is particularly suitable for short-core-length motors with weak PMs because the axial dc flux produced by the brushless stationary field winding can enter one end of the rotor axially and then distribute in the radial air gap for entry into the stator core. If the core length is long, the radial air gap area per pole can be much greater than the axial area per pole where the axial dc flux enters the rotor. For a given axial flux, the greater the radial area, the lower the radial flux density; hence there will be a weaker influence of the excitation current on the radial air-gap flux density.

The RIPM motor without external field excitation coils demonstrated a technology for increasing the air-gap PM flux density when side PMs are used. The air-gap flux density reached 1.2 Tesla, slightly exceeding the $\mathrm{Br}$ of the PMs used in the rotor. This may open a door for further improvement in motor development with RIPM and RIPM-BFE motors.

Test results for corona-initiating voltages for mush-wound and bobbin-wound windings were experimentally obtained. On the basis of the corona tests, for high-voltage motor designs, the insulation system, especially for mush-wound windings, needs to be closely examined because of low phase-tophase corona-initiating voltages.

\section{Future Direction}

The drive motor is an extremely important component in either a fuel-cell vehicle or an HEV. From a systems perspective, motor research must take all the components of the drive system into consideration. The RIPM-BFE motor was developed to simplify the boost requirement and to offer the possibility of using weaker and cheaper PMs. Other system issues still need to be examined; for instance, batteries 
supplied by foreign manufacturers for HEVs cannot meet the demands of U.S. car makers. Motor designs that lower the demand on the batteries could be an important research area.

Further engineering optimization of the RIPM-BFE motor design is necessary to overcome magnetic saturation issues. This motor is particularly suitable for a short-core-length machine that can significantly transfer the axial-direction flux produced by the brushless field excitation coil to the radial air-gap flux. Designs using this motor technology have the potential to use weaker, less costly PMs. Future designs that assess the feasibility of using injected, bonded magnets in this machine and higher-speed motors may very well yield less costly motors with higher performance capabilities.

A new concept for a motor design with side magnets can provide a machine with a stronger fixed PM air-gap flux density. This technology can be used in either the RIPM-BFE or the RIPM motor and can result in a higher PM air-gap flux density for both strong and the low-cost weak magnets.

\section{Patents}

The available ORNL patents and patents pending on the RIPM-BFE machine and the HSUPM machines are as follows:

- "High Strength Undiffused Brushless Electric Motors and Generators," U.S. Patent 6,573,634, June 3, 2003.

- "Hybrid-Secondary Uncluttered Induction Machine," U.S. Patent No. 6,310,417, October 30, 2001.

- "Simplified Hybrid-Secondary Uncluttered Induction (HSU-I) Machine," U.S. Patent 6,891,301, May 10, 2005.

- "Hybrid-Secondary Uncluttered Permanent-Magnet (HSU-PM) Machine," patent pending no. 10/706,577.

- "Improvements on High Strength Undiffused Machine," patents pending nos. 60/675,419, and $11 / 162753$.

- "HSUB Reluctance Machine," patent pending no. 11/019,075.

- "Compact HSUB Electric Motor/Generator," patent pending no. 10/848,450.

- "High Slot Utilization Systems for Electric Machines," patent pending no. 0813-01-100305.

- "Motor with Low Battery Requirement," patent pending No. 1636-01-100405. 


\title{
3.3 Advanced Traction Motor Development (RFP)
}

\author{
Principal Investigator: Sam Nelson \\ Oak Ridge National Laboratory \\ National Transportation Research Center \\ 2360 Cherahala Boulevard \\ Knoxville, TN 37932 \\ Voice: 865-946-1327; Fax: 865-946-1262; E-mail:neslonsscjr@ornl.gov \\ DOE Technology Development Manager: Susan A. Rogers \\ Voice: 202-586-8997; Fax: 202-586-1600; E-mail: Susan.Rogers@ee.doe.gov \\ ORNL Program Manager: Mitch Olszewski \\ Voice: 865-946-1350; Fax: 865-946-1262; E-mail: olszewskim@ornl.gov
}

\section{$\underline{\text { Objectives }}$}

The objective of this task is to develop advanced traction drive motors that would not readily be pursued by industry alone because of high risks and uncertain or long-term outcomes. The overall objective is to design, develop, fabricate, deliver, and test advanced traction drive motors that will meet the 2010 FreedomCAR goals and traction motor technical targets. The goal of this task is to develop an advanced traction drive motor with improved motor torque capability as well as improved efficiency and power density and reduced cost.

\section{Approach}

A solicitation was developed with qualification requirements for the potential offerors, including goals and specifications (i.e., efficiency, power density, torque, minimal speed requirements) for development of an advanced traction drive motor. A survey was made of potential traction drive motor developers, and a request for proposals (RFP) was sent to potential bidders. The proposals received from traction drive motor bidders were studied and evaluated based on (1) technical approach, (2) corporate experience, (3) personnel qualifications, and (4) project management. The proposal with the greatest value and with the lowest risk for the development of advanced traction drive motors was selected by the evaluation team.

Phase 1 of the task is to perform the research and development (R\&D) required, resulting in a design for an advanced traction drive motor. Phase 2 of the task is to fabricate and deliver for test and evaluation an advanced traction drive motor in accordance with the specifications presented in the solicitation.

\section{Major Accomplishments}

A solicitation was issued to traction drive motor suppliers to determine their interest in developing advanced traction drive motors that will meet 2010 FreedomCAR goals and specifications. The RFP was issued on February 18, 2005, with an initial closing date of March 30, 2005. The RFP resulted in the receipt of four proposals, none of which met all of the qualification criteria. The RFP was amended on June 7,2005 , to modify the qualification criteria with a June 14, 2005, closing date. The amendment did not result in any additional proposals, but three of the proposals met the revised qualification criteria. Site visits were made to each offeror to gain additional information about the proposals and the offeror's capabilities. An evaluation team report was prepared documenting the assessment of each proposal. A Phase 1 contract was awarded in August 2005 to UQM Technologies, Inc., to design an advanced traction drive motor. 


\section{Technical Discussion}

Oak Ridge National Laboratory (ORNL) motor scientists are being used during the procurement and performance period of this task to enhance and complement the supplier's technical capabilities. This task included the development of a solicitation with specifications and goals for the advanced traction drive motor. The advanced traction drive motor is to have a $30-\mathrm{kW}$ continuous and $55-\mathrm{kW}$ peak power capability. The motor is to have a constant power speed ratio (CPSR) of 5 with a minimum top speed of $10,000 \mathrm{rpm}$ and an efficiency of $93 \%$ from 10 to $100 \%$ of rated speed at $20 \%$ of rated torque. An RFP was issued, and a variety of traction drive motor design technologies were proposed by the offerors. The designs were evaluated, and the design that was considered by the evaluation team to have the greatest value and lowest risk for advanced traction drive development was selected.

Phase 1 design activity was initiated in August by UQM Technologies of Golden, Colorado. To date, the design process has focused on creating parametric models of the Prius motor geometry and verifying the results with ORNL test data for the Prius. The Prius dimensions and material properties parameters have been entered into the models, auto-generated, and solved for back-electromotive force (EMF) waveform shapes and amplitude. Inductance models for the Prius have also been created and solved. The custom element mesh for the parametric Prius-style model is shown in Figure 1, along with the no-load flux distribution.
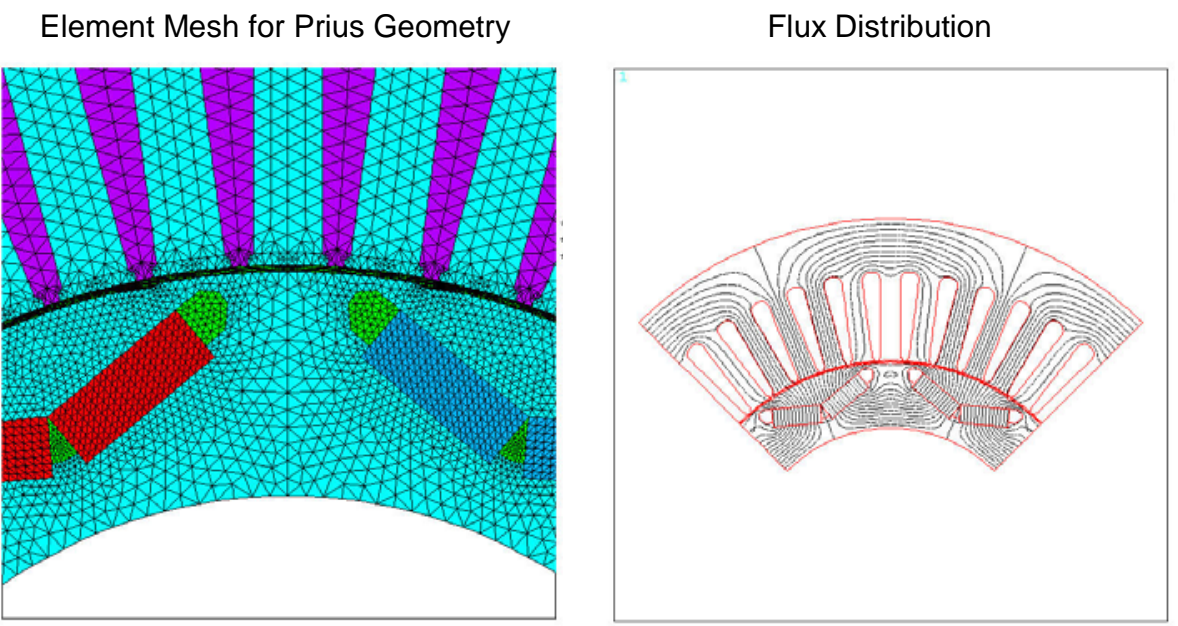

Figure 1. Element mesh for Prius geometry and flux distribution.

The back-EMF waveform and magnitude can be calculated accurately by solving for the flux distribution at several different rotor angles. The back-EMF waveform predicted by finite element analysis for the Prius motor is illustrated in Figure 2. The predicted back-EMF constant for the Prius motor was $140.5 \mathrm{~V}_{\mathrm{LL}} / \mathrm{krpm}$, which correlated closely with the measured results from ORNL, 141.7 $\mathrm{V}_{\mathrm{LL}} / \mathrm{krpm}$. 


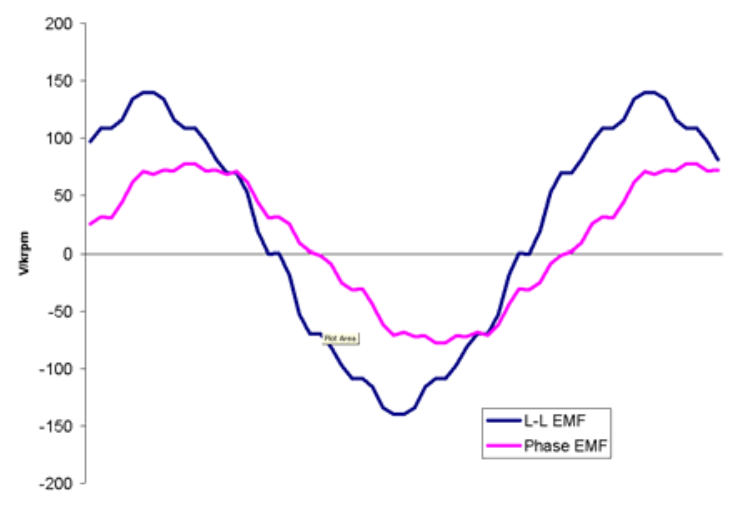

Figure 2. Predicted back-EMF waveform of the Toyota Prius traction drive motor.

\section{Conclusion}

ORNL motor scientists will work closely with UQM Technologies during the R\&D tasks associated with the design of the traction drive motor. Phase 1 will involve detailed analysis and design, a cost study, and the analysis of potential processes, materials, configurations, and manufacturing methods that will meet the requirements for an advanced traction drive system that will meet the solicitation specifications and the 2010 FreedomCAR goals.

\section{Future Direction}

During FY 2006, this task will result in the development of an advanced traction drive motor design. The progress and merits of the Phase 1 design activity will determine if a Phase 2 task is funded. A Phase 2 task would require the fabrication and delivery of a prototype traction drive motor system for test and evaluation. 


\section{Power Electronics Research and Technology Development}

\subsection{Wide-Bandgap Semiconductors}

Principal Investigator: Burak Ozpineci

Oak Ridge National Laboratory

National Transportation Research Center

2360 Cherahala Boulevard

Knoxville, TN 37932

Voice: 865-946-1329; Fax: 865-946-1262; E-mail: ozpinecib@ornl.gov

DOE Technology Development Manager: Susan A. Rogers

Voice: 202-586-8997; Fax: 202-586-1600; E-mail: Susan.Rogers@ee.doe.gov

ORNL Program Manager: Mitch Olszewski

Voice: 865-946-1350; Fax: 865-946-1262; E-mail: olszewskim@ornl.gov

\section{Objectives}

- Assess the impact of replacing silicon ( $\mathrm{Si}$ ) power devices in transportation applications with devices based on wide-bandgap (WBG) semiconductors, especially silicon carbide (SiC)-based ones.

- Develop device models for system-level simulation studies and analyze the impact of SiC devices on the system performance.

- Build and test SiC-based power converters for hybrid electric vehicles (HEVs) to validate the predictions.

- $\quad$ Test the reliability of the $\mathrm{SiC}$ devices.

\section{Approach}

- Maintain an awareness of the state of the art in WBG semiconductors.

- Develop models of WBG semiconductor devices, especially SiC diodes, junction field-effect transistors (JFETs), and metal oxide semiconductor field-effect transistors (MOSFETs).

- Simulate the performance of an HEV traction drive and a dc-dc converter using these device models.

- Test, characterize, and model a 55-kW Si-SiC hybrid [Si-insulated gate bipolar transistor (IGBT), SiC Schottky diodes] inverter and compare it with a similar all-Si inverter.

- Test, characterize, and model a 7.5-kW all-SiC inverter to compare with an all-Si inverter and validate the system models and demonstrate the system-level benefits.

- $\quad$ Build dc-dc converters to test the reliability of the SiC and Si devices.

\section{Major Accomplishments}

- Acquired several SiC Schottky diodes, JFETs, and MOSFETs.

- Tested, characterized, and modeled SiC Schottky diodes, JFETs, and MOSFETs. Physics-based models for JFET and SiC Schottky diodes were also developed in Saber.

- Tested, a 55-kW Si-SiC hybrid traction inverter built in collaboration with Cree and Semikron and compared its performance with a 55-kW all-Si inverter. Both were modeled in Saber using the device models developed from the device characterization data.

- Tested a 7.5-kW all-SiC inverter (SiC JFETs and SiC Schottky diodes) that was built in collaboration with Rockwell Scientific and compared its performance with a commercially available all-Si inverter 
(Si IGBTs and Si pn diodes). The all-SiC inverter was modeled in Saber using the JFET models and SiC Schottky diode models.

- Tested the reliability of SiC and Si devices by using them in buck converters and operating the buck converters for long periods of time.

\section{Technical Discussion}

With the increase in demand for more efficient, higher-power electronics with higher-temperature capabilities, design engineers face new challenges [1, 2]. Silicon (Si) power devices have reached their theoretical limits in terms of higher-temperature and higher-power operation by virtue of the material's physical properties. To overcome these limitations, research has focused on wide-bandgap materials such as silicon carbide $(\mathrm{SiC})$, gallium nitride $(\mathrm{GaN})$, and diamond because of their superior material advantages such as large-bandgap, high-thermal conductivity, and high-critical breakdown field strength.

\section{SiC Field-Effect Transistor Devices}

FET devices are majority-carrier devices and are preferred over minority-carrier devices in power applications; however, Si FET devices, like Si Schottky diodes, can be used only in low-voltage $(<300$ V) applications because of their high on-state resistance. Even the first experimental SiC FET devices have blocking voltages over $1000 \mathrm{~V}$ with reasonable on-state resistances. It is expected that in the near future SiC FET devices will dominate Si minority-carrier devices in medium-voltage $(<3000 \mathrm{~V})$ applications.

\section{SiC MOSFET}

\section{Static characteristics}

A MOSFET is a unipolar device that is normally off. The forward characteristics of the same $\mathrm{SiC}$ MOSFET for a temperature range of $-50^{\circ} \mathrm{C}$ to $175^{\circ} \mathrm{C}$ are shown in Figure 1 . The on-state resistances of the device are calculated from the slopes of the curves and are plotted with respect to temperature in Figure 2. It is interesting to note that this device has a negative temperature co-efficient up to $50^{\circ} \mathrm{C}$ and a positive temperature coefficient above that. MOSFETs are majority-carrier devices and are expected to have positive temperature coefficients. The reason for this is that at lower temperatures, the contribution of the channel resistance to the total on-state resistance is dominant [3,4]. The channel mobility increases with temperature because the interface traps are closer to the conduction band [4,5]; thus, the channel resistance decreases with temperature. Consequently, at low temperatures the MOSFET on-resistance decreases. Above a certain temperature value, the channel resistance is not dominant, and because of the other dominant on-resistance components, the MOSFET overall on-resistance increases.

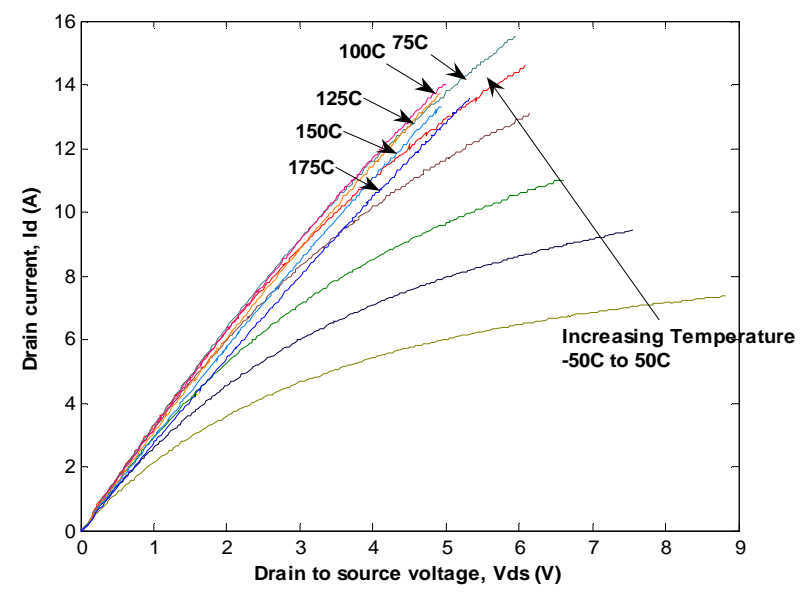

Figure 1. Forward characteristics of SiC MOSFET at different temperatures. 


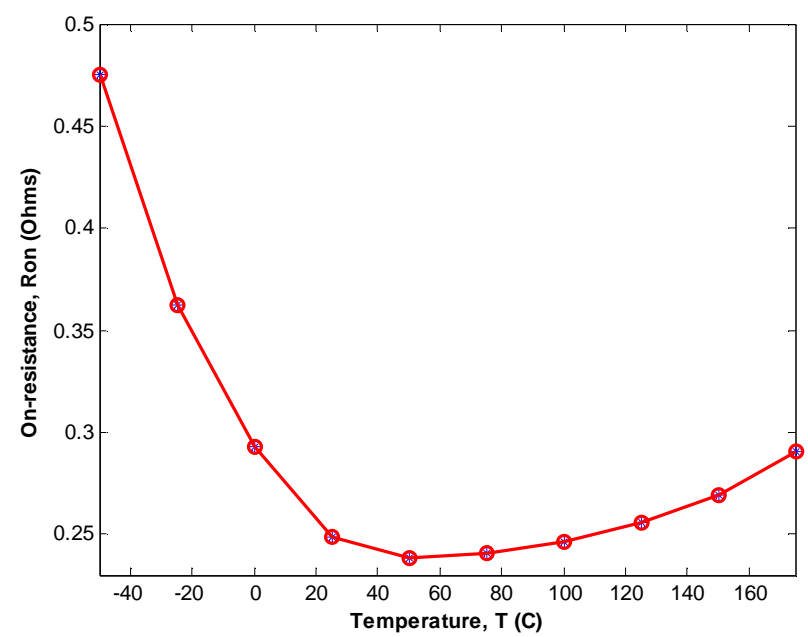

Figure 2. On-resistance of SiC MOSFET at different temperatures.

\section{Dynamic characteristics}

The gate-drive circuit developed at ORNL in FY 04 for SiC JFET was modified and used to determine the dynamic characteristics of the SiC MOSFET. The gate-drive voltage for the MOSFET was selected to be $20 \mathrm{~V}$ as determined from the forward characteristics to obtain the optimum performance. Operation at $250 \mathrm{kHz}$ was achieved with a series-gate resistance of $7.2 \Omega$ and a peak-gate current of $0.6 \mathrm{~A}$. The gate and switching waveforms for a SiC MOSFET are shown in Figure 3. The device has a turn-off delay td of $40 \mathrm{~ns}$, fall time tf of $100 \mathrm{~ns}$, turn-on delay td of $20 \mathrm{~ns}$, and rise time tr of $100 \mathrm{~ns}$.

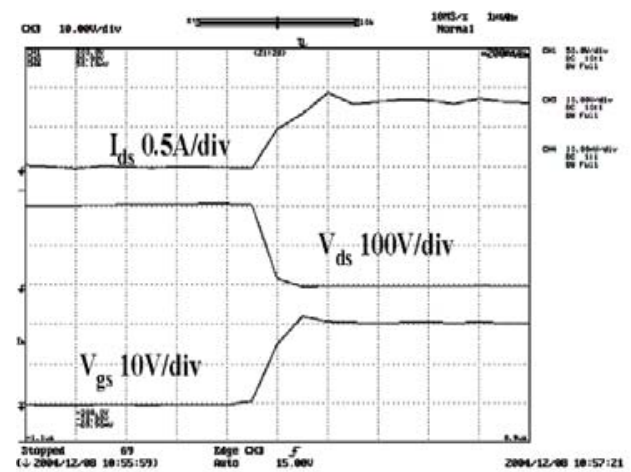

(a)

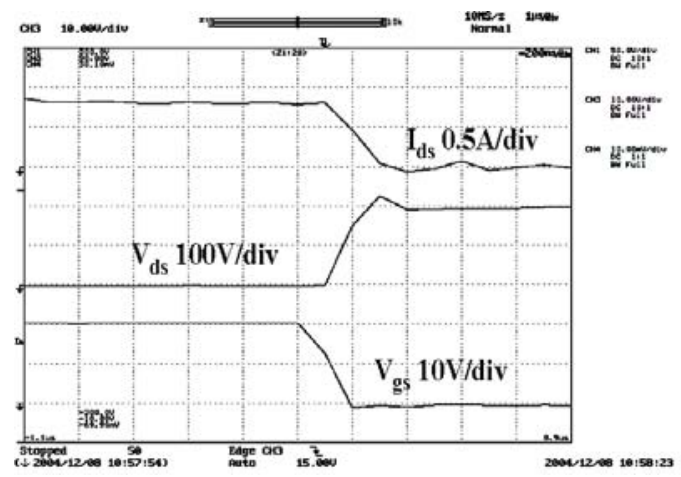

(b)

Figure 3. The gate and switching waveforms of the SiC MOSFET. 
MOSFETs have fast dynamic responses, which are required for high-frequency switching. The turn-on and turn-off energy losses for the SiC MOSFET were calculated by integrating the instantaneous power over the turn-on (ton) and turn-off times (toff). The energy losses calculated for the SiC MOSFET at different temperatures for operation at $5 \mathrm{kHz}, 50 \%$ duty cycle, $100 \mathrm{~V}$, and $0.8 \mathrm{~A}$ are shown in Figure 4. It is evident from the plot that the total switching losses do not vary much with temperature.

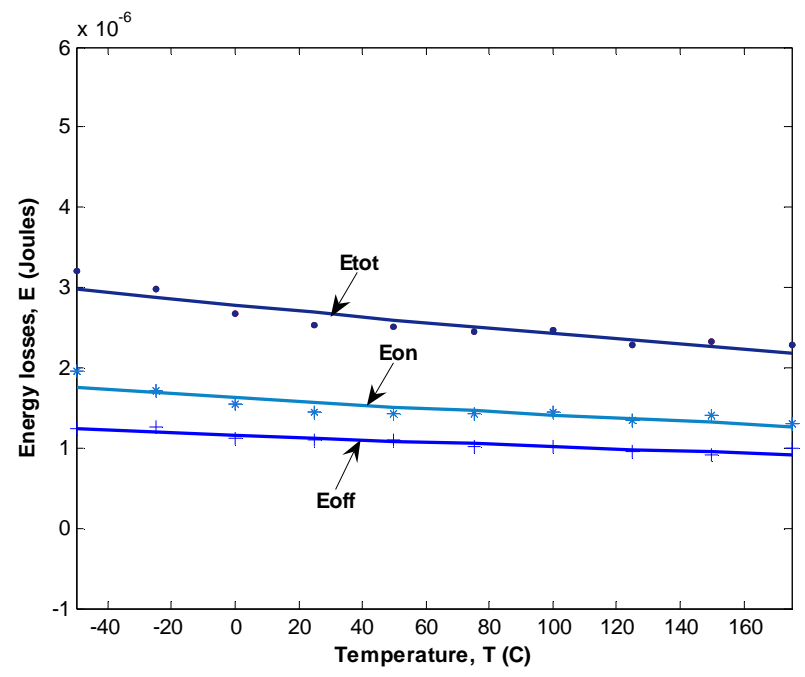

Figure 4. Energy loss plot of SiC MOSFET.

\section{5-kW Si-SiC Hybrid Inverter}

SiC Schottky diodes have been proven to have better performance characteristics than those of similar Si pn diodes [6], especially with respect to their switching characteristics because they have negligible reverse-recovery losses. Their superior switching performance impacts the main power switches by reducing the stress on them and thus improving system performance. SiC Schottky diodes are already commercially available at low-current ratings. These diodes are being used in niche applications such as power factor correction circuits. It is expected that the first impact of $\mathrm{SiC}$ devices on inverters will be as a result of SiC Schottky diodes replacing the Si pn diodes.

Oak Ridge National Laboratory (ORNL) collaborated with Cree and Semikron to build a hybrid 55-kW (Si IGBT-SiC Schottky diode) inverter by replacing the Si pn diodes in Semikron's automotive inverter with Cree's SiC Schottky diodes.

The inverter module SKAI (Semikron Advanced Integration) from Semikron was developed for hybrid electric vehicle (HEV) traction drives. It is a three-phase $55-\mathrm{kW}$ inverter unit (Figure 5) built using 600-V, 600-A IGBTs and 600-V, 450-A pn diode modules. Cree has developed 75-A, 600-V, $4 \times 6.65$ $\mathrm{mm} \mathrm{SiC} \mathrm{Schottky} \mathrm{diodes} \mathrm{as} \mathrm{shown} \mathrm{in} \mathrm{Figure} \mathrm{6.} \mathrm{The} \mathrm{yield} \mathrm{was} \mathrm{about} 46.7 \%$, with 67 good devices from the wafer. For this project, Semikron replaced each Si pn diode $(9 \times 9 \mathrm{~mm})$ in their automotive integrated power module (AIPM) with two 75-A SiC Schottky diodes. 


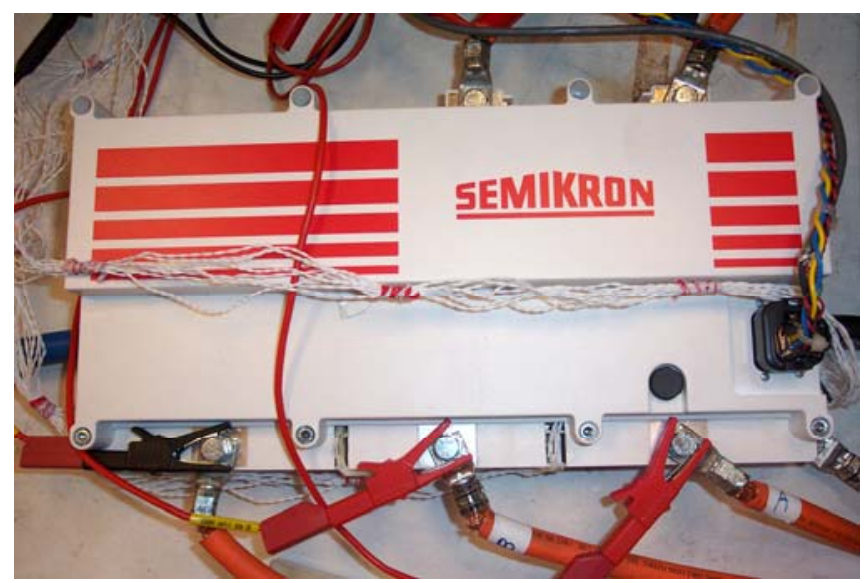

Figure 5. Semikron inverter unit.
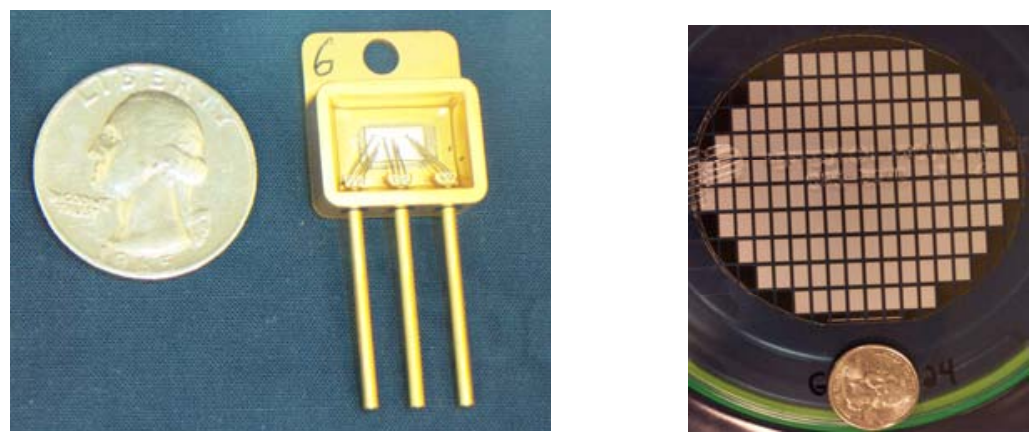

Figure 6. 75-A Schottky diodes developed by Cree.

\section{SiC diode modeling}

The SiC Schottky diode model used in this project was developed at the University of Arkansas. It is a robust model that can accurately model forward and reverse recovery and track accurately over temperature. Moreover, many details pertaining to the fabrication of the diode need not be known by the user, making parameter extraction flexible. The diode model parameters were extracted from the on-state characteristics, and from the reverse-recovery waveforms.

The on-state validation of the model with the $\mathrm{SiC} 75$-A diode from Cree is seen in Figure 7. The percentage error is approximately $0.3-0.4 \%$ in the $100^{\circ} \mathrm{C}$ and $150^{\circ} \mathrm{C}$ curves and approximately $2-3 \%$ in the $25^{\circ} \mathrm{C}$ curve.

Figure 8 shows the reverse recovery of the $\mathrm{SiC}$ diode and the corresponding behavior of the model (in dotted lines). As seen in the figure, the model accurately tracks the test data.

\section{Simulation of Si IGBT-SiC Schottky diode hybrid inverter}

After the modeling and parameter extraction of the devices, the device models were used to construct a three-phase voltage-source inverter in Saber. The IGBT that was used for constructing the Semikron inverter was not available in the Saber library. Therefore, another IGBT with similar ratings was used as the transistor switch. The load consisted of a $1.24 \Omega$ resistor and a $1.5 \mathrm{mH}$ inductor and the input dc bus voltage was set at $325 \mathrm{~V}$. An all-Si inverter with Si IGBTs and diodes was also simulated. The simulation results showed that the Si IGBT- SiC Schottky diode hybrid had much better efficiencies than the all-Si inverter. 


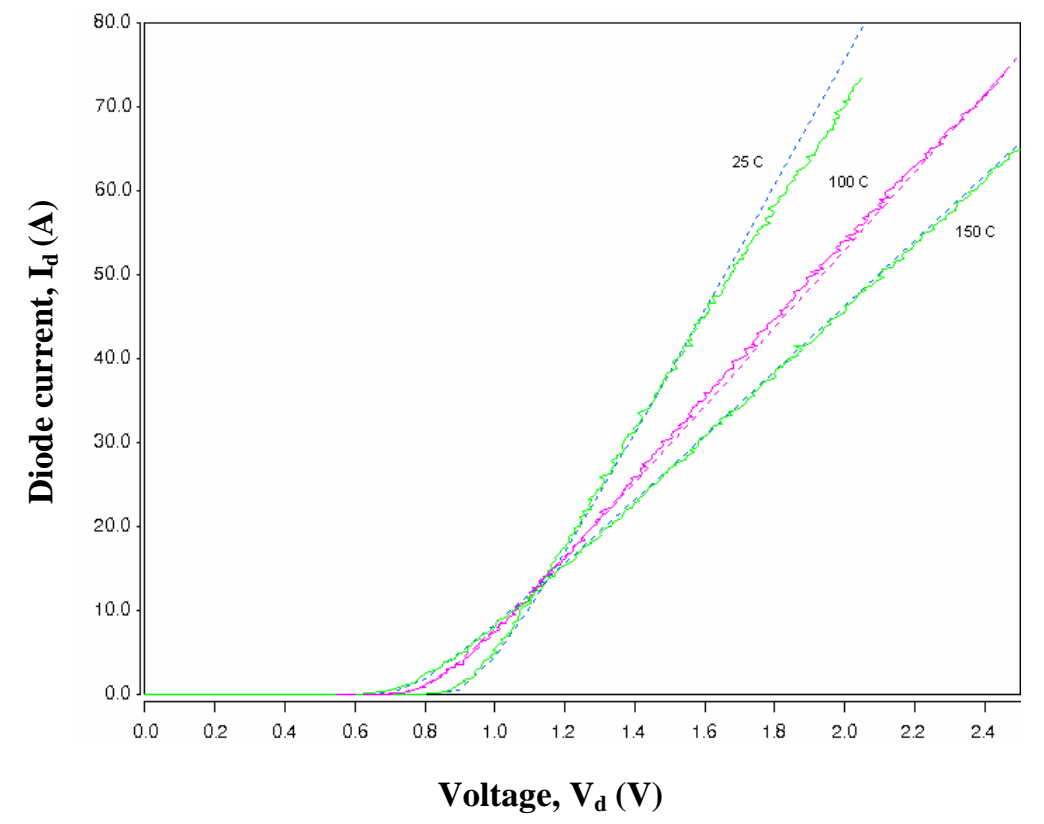

Figure 7. Measure (solid line) and simulated (dotted line) on-state waveforms of SiC Schottky diode at different temperatures.

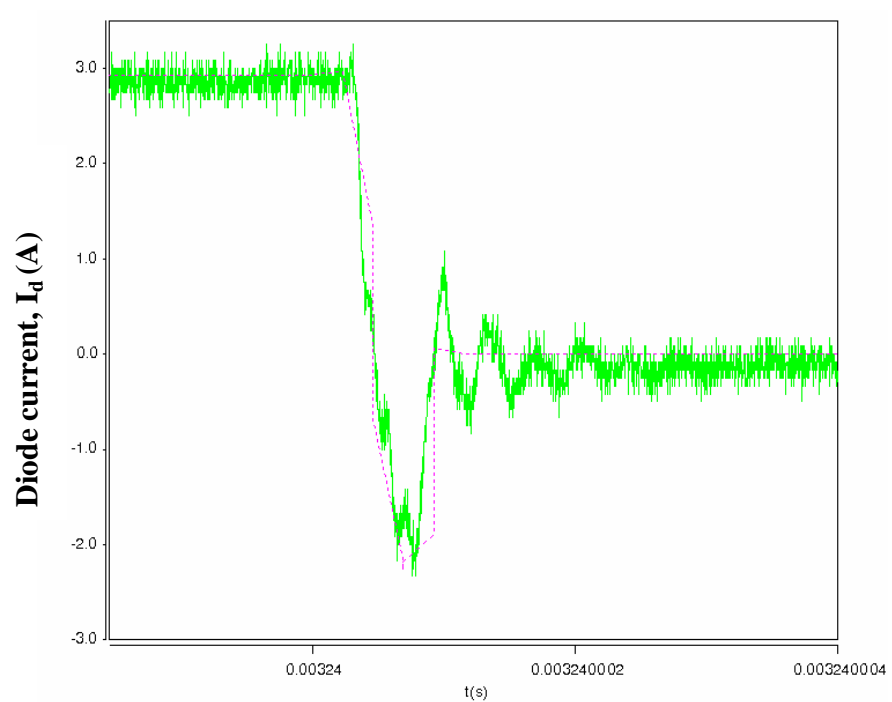

Figure 8. Measured (solid) and simulated (dotted) reverse-recovery waveforms of the SiC Schottky diode from Cree.

The difference in efficiencies between the simulated inverters is clearly visible in Figure 9. When the $\mathrm{Si}$ diode in the all-Si inverter was replaced with the SiC Schottky diode, a 1.5-2\% increase in the operational efficiency was observed.

Two major tests were performed, R-L load tests to investigate the inverters power handling capability and dynamometer tests to determine the inverters dynamic performance. Both the hybrid inverter and the all-Si inverter were tested using the same test procedure. 


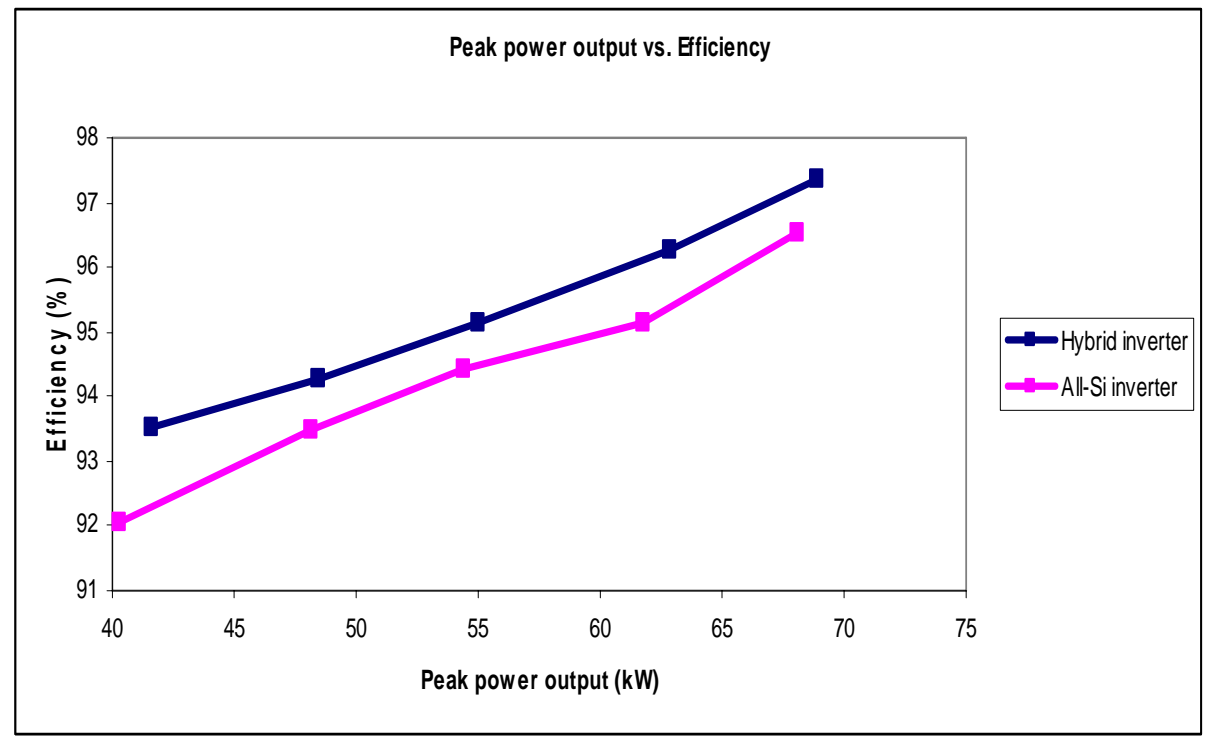

Figure 9. Comparison of peak-power output vs efficiencies for hybrid and all-Si inverter testing.

\section{R-L load test}

Operation. For this test, the dc-link voltage was varied from the minimum operating voltage (200 V) to the maximum bus voltage $(450 \mathrm{~V})$. The bus voltage trip fault occurs for voltages beyond $450 \mathrm{~V}$. The load resistance was set to the minimum value and the current was controlled using the current controller. The coolant was set at $20^{\circ} \mathrm{C}$ at a flow rate of $2.5 \mathrm{gpm}$. The open-loop frequency of operation and the pulse-width modulation (PWM) frequency were fixed, and the current command was varied for a particular dc-link voltage. The command current was increased in steps of $10 \mathrm{~A}$ without exceeding the power rating of the inverter or the power rating of the load. The procedure was repeated by increasing the open-loop frequency in steps of $25 \mathrm{~Hz}$. The coolant temperature was changed to $70^{\circ} \mathrm{C}$, and the data was recorded for a wide range of current and open-loop frequencies.

Results. The data obtained for both the inverters were analyzed and the corresponding efficiencies were calculated. The efficiency versus output-power plot for one of several operating conditions comparing the inverters is shown in Figure 10. The hybrid inverter efficiencies are higher than the all-Si inverter for all operating conditions. The results show up to $33.6 \%$ reduction in the losses when everything is kept the same and SiC Schottky diodes are used instead of Si pn diodes. The percentage loss reduction was calculated comparing the power losses between the hybrid inverter and the all-Si inverter as follows:

$$
\text { Percentage loss reduction }=\left(d_{\text {ploss (Si) }}-d_{\text {ploss (SiC) }}\right) /\left(d_{\text {ploss (Si) }}\right) \times 100
$$

\section{Dynamometer tests}

Motoring mode. The dc-voltage input to the inverter was set at the nominal battery operating voltage $(325 \mathrm{~V} \mathrm{dc})$. The direction of rotation was set to forward and the motor speed was increased from $750 \mathrm{rpm}$ to the rated base speed for a specific continuous load torque. Data was obtained for a wide range of speed and torque values by varying the load torque (from 100, 150, $200 \mathrm{Nm}$ ) using the dynamometer controller.

The efficiency plots for various speeds and a load torque of $200 \mathrm{Nm}$ are shown in Figure 11. The average loss reduction in motoring mode was up to $10.7 \%$. Note that one of the reasons the average loss reduction is less in the motoring compared to the R-L load test is that the power levels achieved are much less in motoring. 


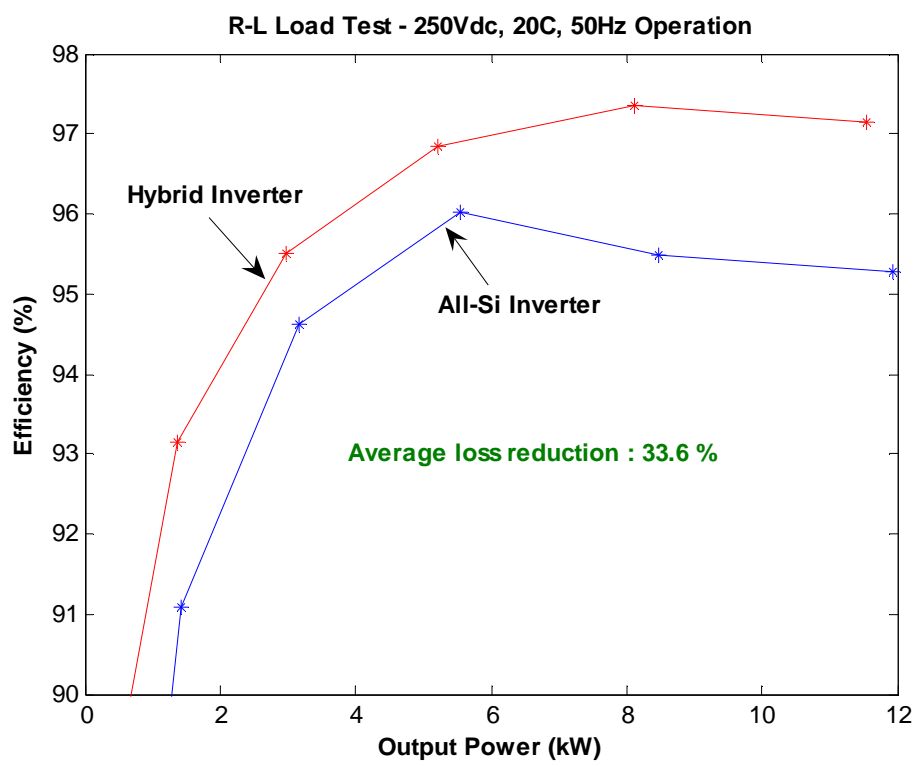

Figure 10. R-L load-test efficiency curves for one of the load conditions.

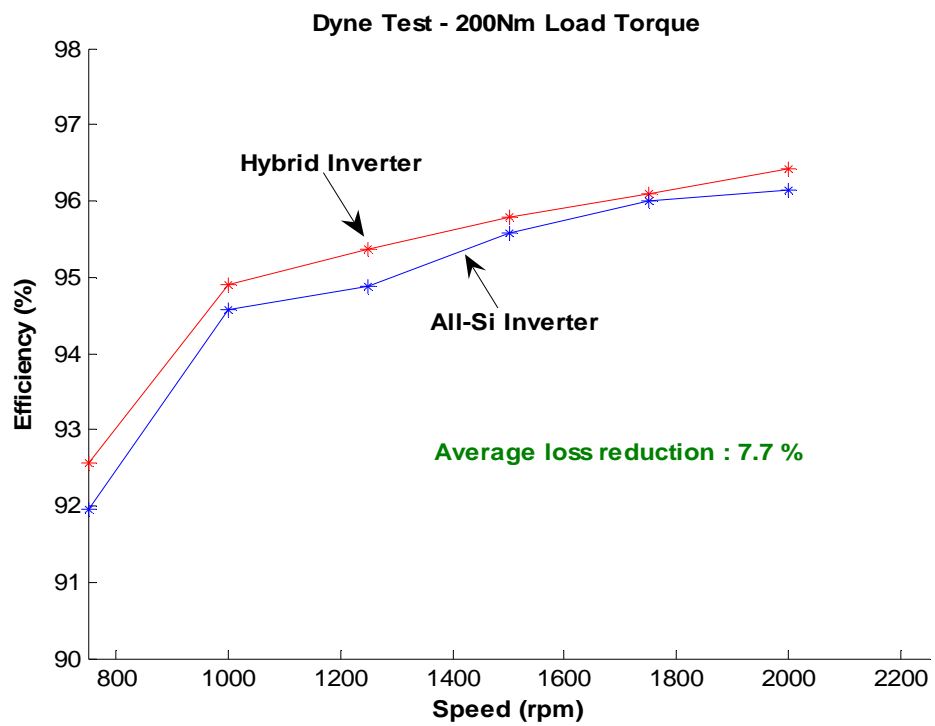

Figure 11. Dynamometer test-motoring mode efficiency plots at $70^{\circ} \mathrm{C}$.

Regeneration mode. The dc-voltage input to the inverter was set at the nominal battery-operating voltage. The direction of rotation was set to be forward. The dynamometer controller was adjusted to control the speed. The motor speed was increased from $750 \mathrm{rpm}$ to the rated speed for a specific operating current of the inverter to achieve a specific load torque. The current was varied from zero to the values corresponding to different torque values and then decreased to zero. The procedure was repeated to obtain the data for a wide range of speed and torque values. The curves comparing the efficiency of the inverters at $70^{\circ} \mathrm{C}$ with a $200 \mathrm{Nm}$ load are shown in Figure 12 . The results show up to $12.71 \%$ reduction in average losses similar to the results obtained in the motoring mode. 


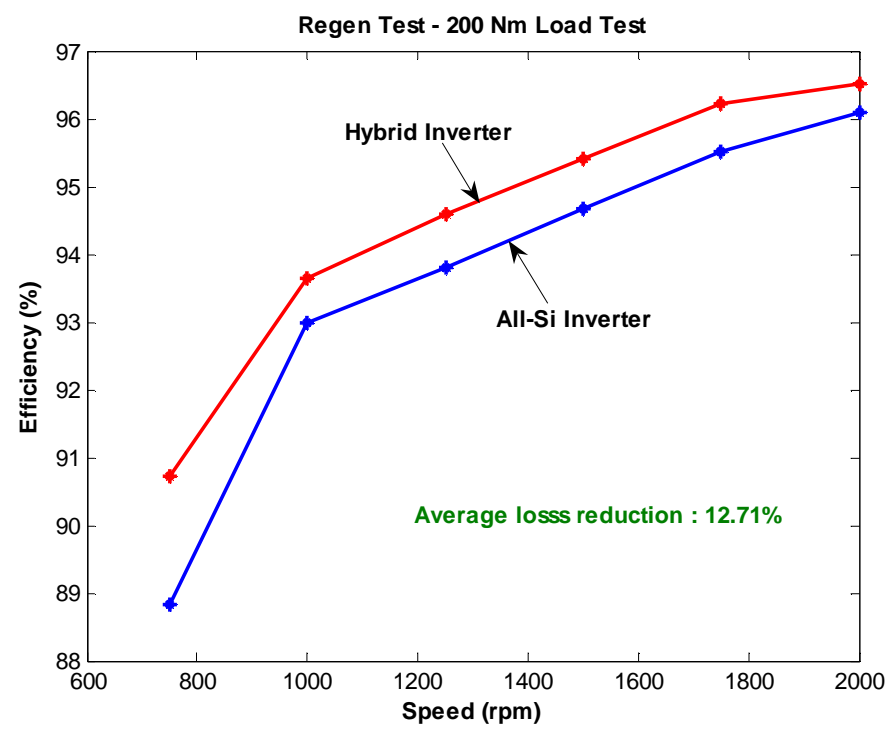

Figure 12. Dynamometer test—regeneration mode efficiency plots at $70^{\circ} \mathrm{C}$.

The IGBTs used in the actual inverter and in the simulation are not the same. Therefore, a direct comparison of the efficiencies of the simulation and the testing would not be accurate. But it is seen that the efficiencies of the simulation and the testing do have the same trend.

\section{5-kW All-SiC Inverter}

A 7.5-kW all-SiC inverter was built in collaboration with Rockwell Scientific to study the impact of replacing the $\mathrm{Si}$ devices with $\mathrm{SiC}$ devices in the inverters. The $\mathrm{SiC}$ inverter module was built using 1200-V, 7.5-A JFETs and 1200-V, 15-A SiC Schottky diodes. The main switches in each phase leg consisted of two JFETs with 7.5-A ratings in parallel.

A Si inverter module with similar ratings was selected to compare its performance with the all-SiC unit. The picture of the $\mathrm{SiC}$ inverter module is shown in Figure 13.

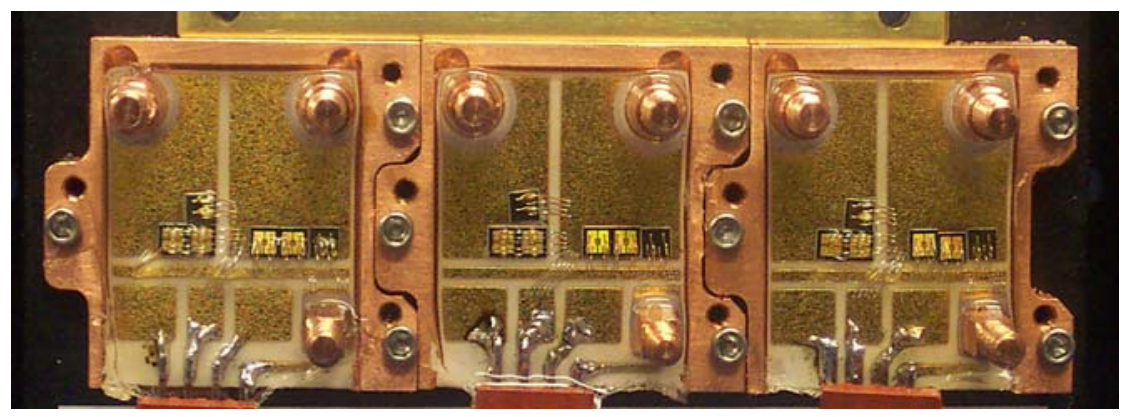

Figure 13. All-SiC module.

\section{SiC JFET modeling}

The device physics-based analytical SiC JFET model used for this project was developed by the University of Arkansas [7]. The on-state validation was done for only the unipolar operation because the JFET is mainly used in that area as shown in Figure 14. The simulated values agree with the measured values with an error of approximately $2-3 \%$. The switching characteristics are shown in Figures 15 and 16. The model tracks the actual waveforms as expected. As observed in the transient modeling results, the switching time is on the order of $0.1 \mu$ s for a $25 \Omega$ gate resistance, and the various depletion capacitances 


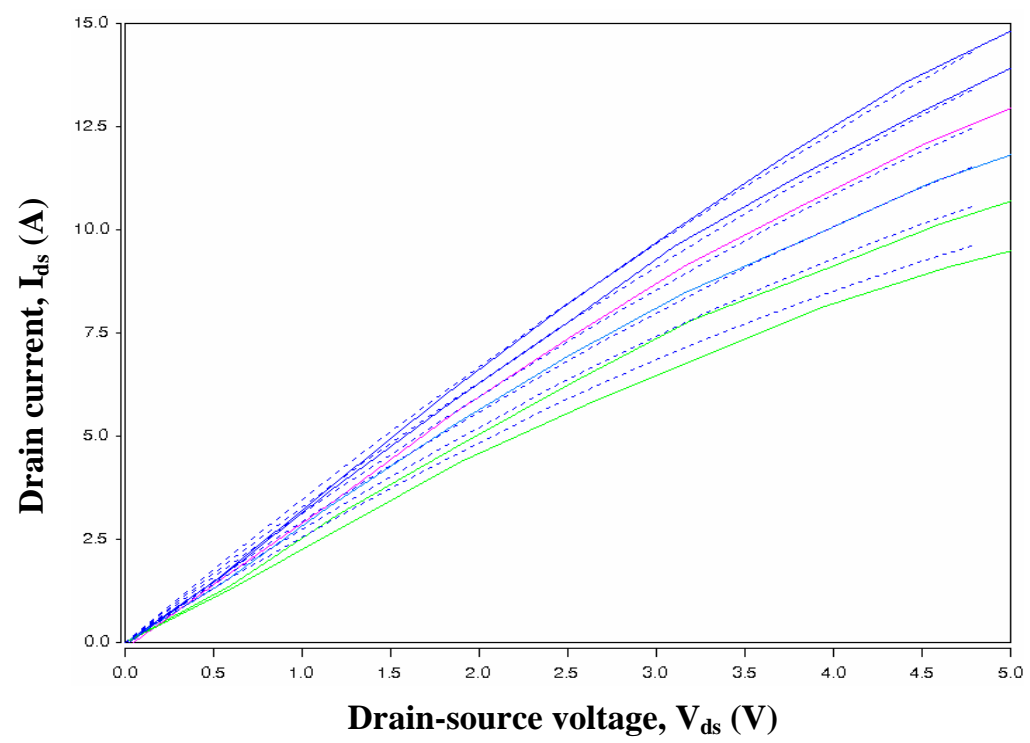

Figure 14. Rockwell SiC JFET measured (solid) and simulated (dotted) on-state waveforms at $25^{\circ} \mathrm{C}$ for different gate voltages $\left(V_{g s}\right.$ ranging from $0 \mathrm{~V}$ to $\left.-2.5 \mathrm{~V}\right)$.

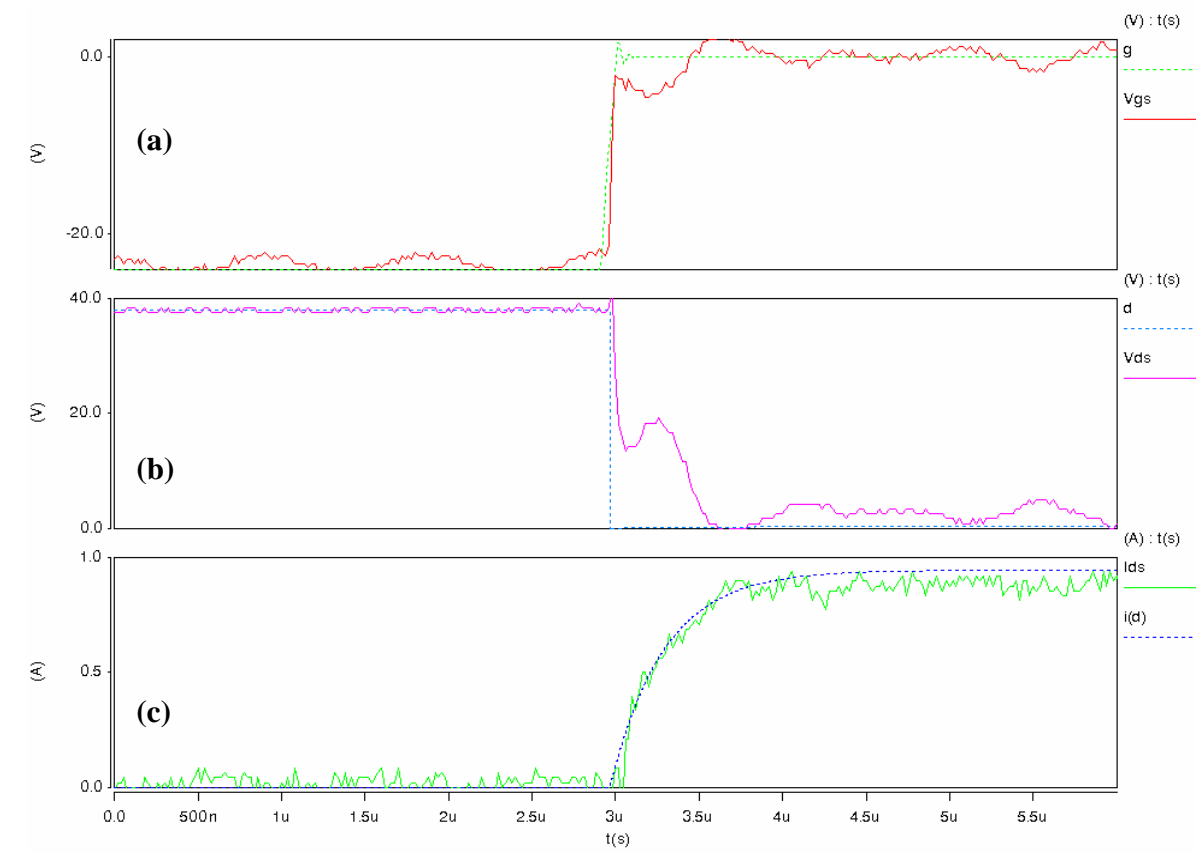

Figure 15. SiC JFET simulated (dashed) and measured (solid) turn-on waveforms at 25oC: (a) gate voltage, (b) drain voltage, and (c) drain current. 


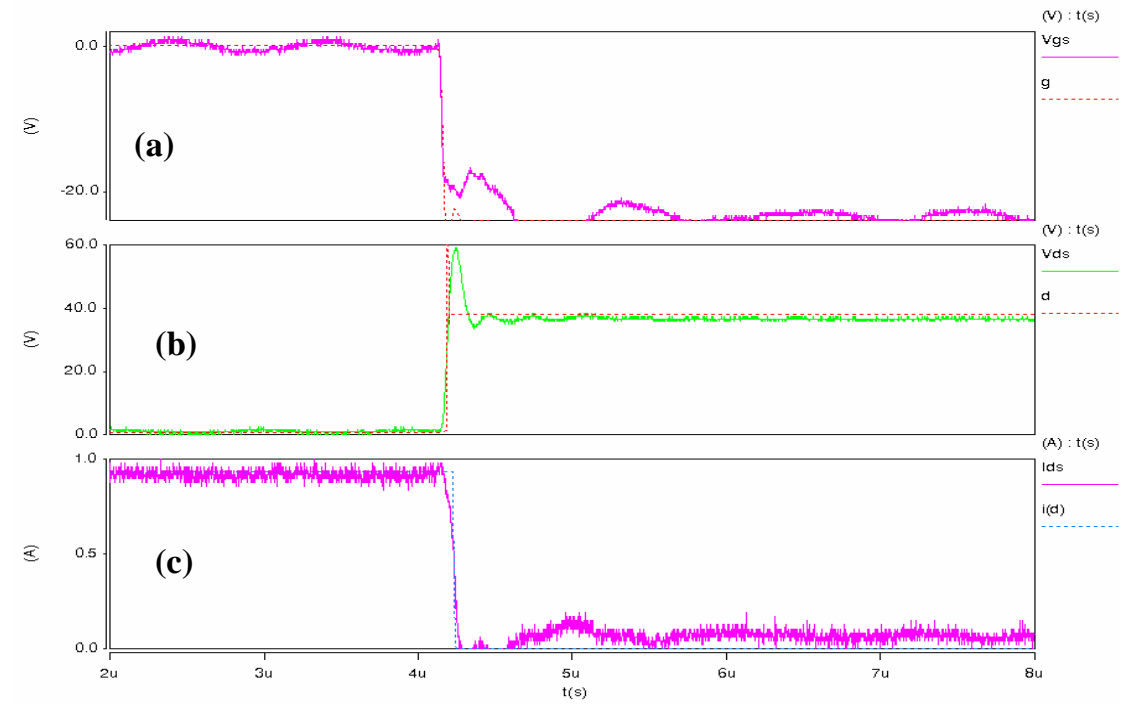

Figure 16. SiC JFET simulated (dashed) and measured (solid) turn-off waveforms at $25^{\circ} \mathrm{C}$ : (a) gate voltage, (b) drain voltage, and (c) drain current.

described in the model can accurately predict the switching characteristics of the SiC JFET. The turn-off waveforms are seen to be much faster compared to the turn-on times because the discharging of the junction and package capacitances happens over a shorter interval of time.

\section{Simulation of all-SiC inverter}

The SiC JFET and diode models were used to construct an all-SiC inverter. This is a technology demonstrator that shows a full power electronic system in $\mathrm{SiC}$ being successfully simulated. The validated models were used to construct the inverter system. The all-SiC inverter from Rockwell was first tested to ascertain the performance and collect test data for the inverter model validation.

A balanced three-phase $15 \Omega$ resistive load was used to simulate the operating conditions. The switching frequency was set to $10 \mathrm{kHz}$, and the output frequency was set to $50 \mathrm{~Hz}$. The inverter was simulated for different values of dc voltages and modulation indices. As explained before, the SiC JFETs used in the inverter were modeled and all the parameters were extracted. The 1200-V, 15-A SiC diode in the inverter was also modeled by the procedure explained previously. The results of the diode fitting are seen in Figures 17 and 18.

The efficiency of the all-SiC inverter was seen to range from 83.2 to $87.8 \%$.

\section{Inverter testing}

\section{Inductive load tests}

Operation. The gate signals to the inverter modules were checked with no load on the inverter. The inverters were connected to a three-phase R-L load. The operating current was controlled by varying the load resistance. The dc-link voltage was varied from 0 to a maximum bus voltage of $400 \mathrm{~V}$. The frequency of operation was fixed and the load was varied for a particular dc-link voltage. The load was increased in steps without exceeding the power rating of the inverter and also not exceeding the power rating of the load. The procedure was repeated by increasing the output frequency command in steps of $10 \mathrm{~Hz}$. 


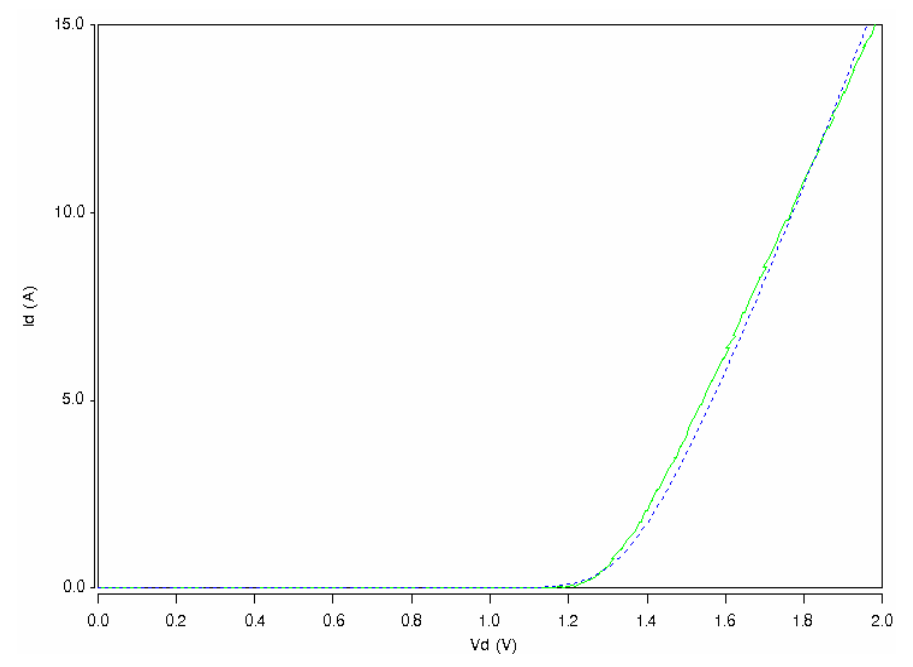

Figure 17. On-state validation of $\mathrm{SiC}$ diode at $25^{\circ} \mathrm{C}$ - measured (solid) and simulated (dashed).

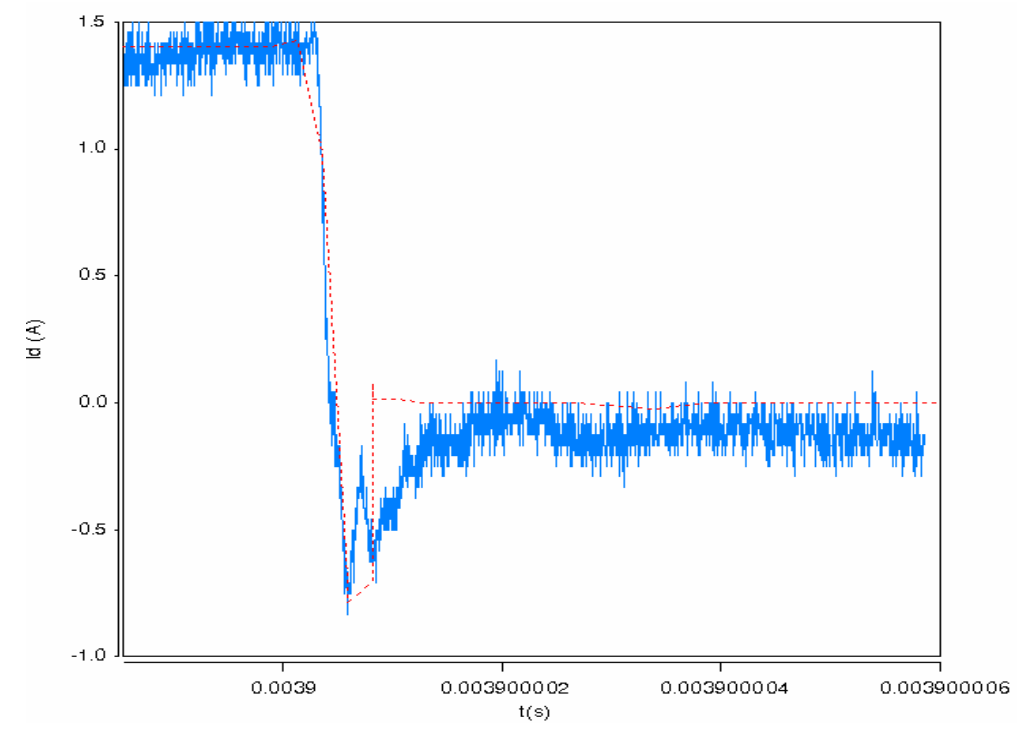

Figure 18. Reverse-recovery validation of $\mathrm{SiC}$ diode at $25^{\circ} \mathrm{C}$ - measured (solid) and simulated (dashed).

Results. The operating waveforms for the $\mathrm{SiC}$ inverter for a particular load setting are shown in Figure 19. The plot of efficiency curves for one of the different operating conditions is shown in Figure 20. It can be seen from the plot that the efficiencies of the Si inverter module are much higher than those of the $\mathrm{SiC}$ module.

To better understand why the all-SiC inverter efficiencies are worse than the all-Si inverter, the static characteristics of the IGBT, SiC JFET, and the diodes in the inverter modules were compared as shown in Figures 21 and 22 respectively.

The on-state voltage drop of the Si IGBT is much less compared to the SiC JFET at higher-current levels because of the higher on-resistance of the SiC JFET. At lower currents, however, the SiC JFET has a lower on-state voltage drop. The Si pn and SiC Schottky diodes, on the other hand, have similar static characteristics. Because of the higher on-state resistance of the SiC JFETs, the all-SiC inverter has higher conduction losses. 


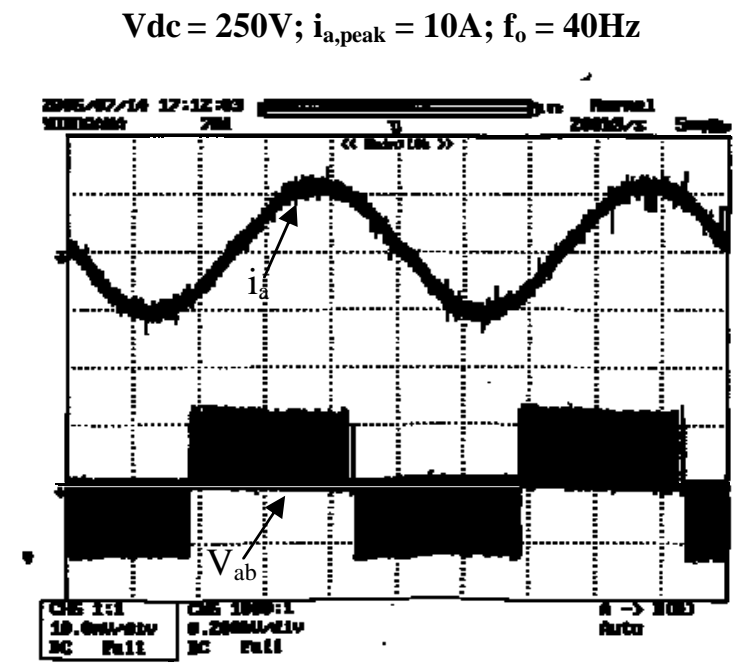

Figure 19. Operating waveforms of SiC module for $\mathrm{R}$-L load test.

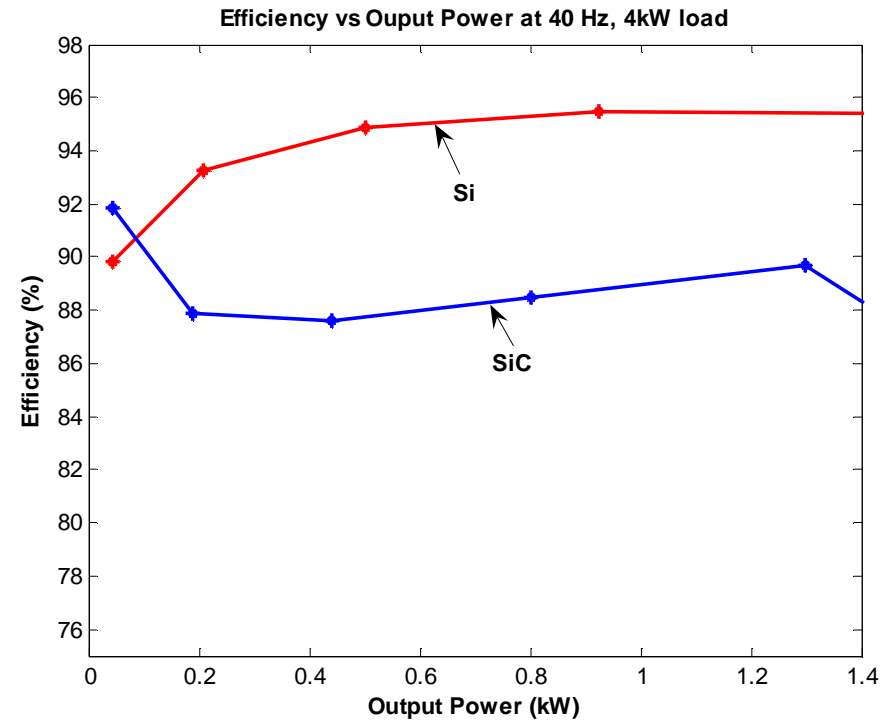

Figure 20. Efficiencies comparison plot at 40-Hz operation for R-L load test. 


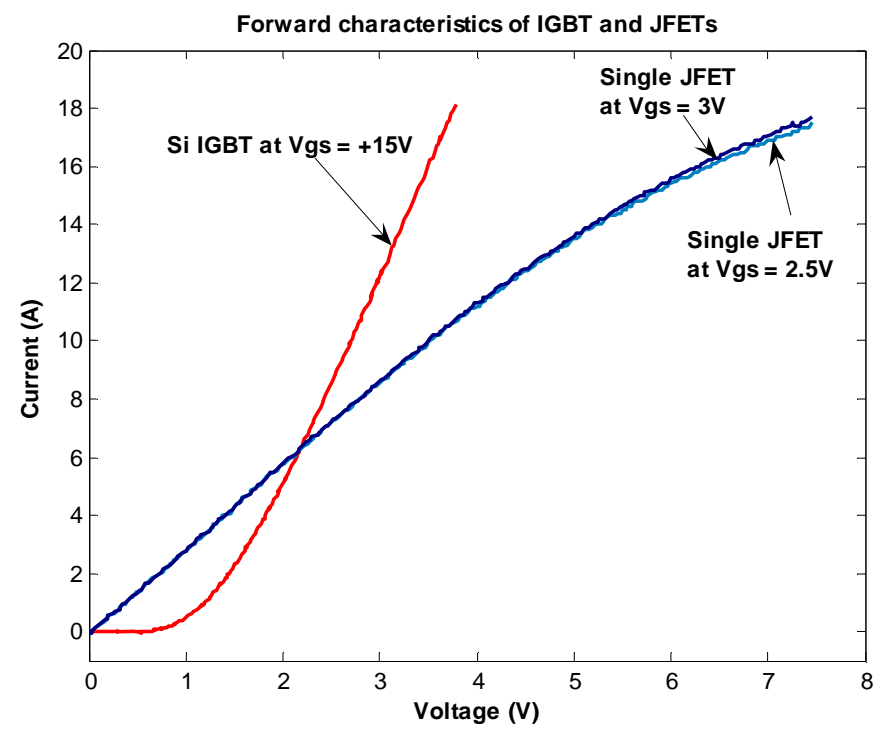

Figure 21. Forward characteristics of IGBT and JFET in the Si and SiC modules.

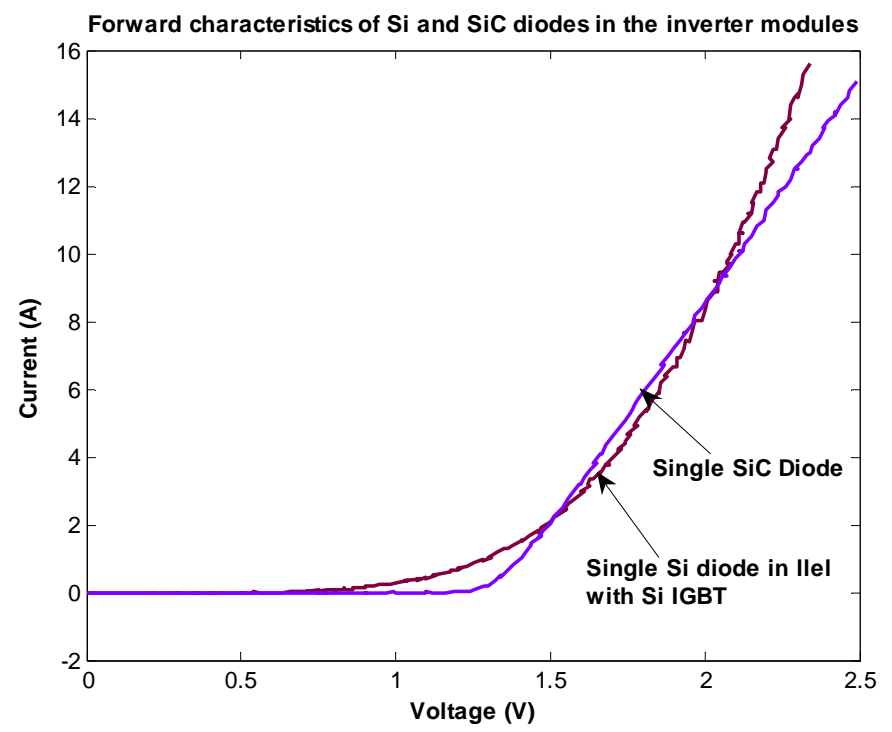

Figure 22. Forward characteristics of diodes in the $\mathrm{Si}$ and $\mathrm{SiC}$ modules. 


\section{Duration tests}

Underhood, reliable operation of power modules in hybrid electric vehicles is an important transportation requirement. Most common failures in power modules are due to power cycling over highoperating temperatures. To test the reliable operation of the SiC power devices, four different buck converters were developed with Si IGBTs, Si pn diodes, SiC JFETs, and SiC Schottky diodes. The devices in the converters were operated at different voltage and current levels and at different frequencies in order to subject them to thermal stress and study their behavior. The analysis of test data and results will be presented in the following sections.

Operation. The buck converters were fed from a single dc supply source, and the load current was varied by changing the input voltage. The operating current for each buck converter was limited to a maximum of 2 A because of the current ratings of the SiC JFETs. These JFETs were obtained through SiCED, a Siemens-based company. They were operated continuously for 7.5 hours every day for six months. During this time, their operation was closely observed and their case temperatures recorded. The temperature profiles have been filtered to get rid of the noise picked up by the thermocouples.

After experimenting with several test conditions, the operating voltage was fixed at $200 \mathrm{~V}$ for safe operation without device failure, and the duration tests were continued. The converters were operated at $10 \mathrm{kHz}, 50 \%$ duty cycle, and $200 \mathrm{~V}$. The temperature profiles recorded for each device for 7.5 hours are shown in Figure 23a. These profiles show that for the same main switch, the SiC diodes are operating at higher temperatures compared to the Si diodes. A previous ORNL simulation study [2] has shown that the conduction losses of SiC Schottky diodes dominate at lower switching frequencies and they are much higher than those of the Si pn diodes. The thermal profiles in Figure 23a confirm the results of that simulation study.

Figure $23 \mathrm{~b}$ shows that $\mathrm{SiC}$ JFETs, without heat sinks, operate at much lower temperatures compared to Si IGBTs, which implies that the SiC devices have lower losses compared to the Si IGBTs. The comparison of static characteristics of SiC JFET and Si IGBT is shown in Figure 24 in which it can be seen that in the current range, the JFET has lower conduction losses.

It is also interesting to observe in Figure 23b that the Si IGBT and the SiC JFET with the SiC diodes operate at lower temperatures compared to the ones that operate with the Si pn diodes. This is again attributed to the better reverse recovery characteristics of the SiC Schottky diodes.

Figures 25 and 26 show all the profiles obtained for different switching frequencies plotted together. It can be observed from these figures that the operating temperatures of the $\mathrm{SiC}$ diodes do not change much with increases in switching frequency. This is because the switching losses of SiC Schottky diodes are significantly lower than those of the Si pn diodes. However, the Si diodes have higher-switching losses and hence their operating temperatures rise as the frequency increases.

Since Si IGBTs are limited in their switching frequency, the buck converters with Si IGBTs were tested separately at lower-switching frequencies. The temperature profiles of the switches and diodes for a $200-\mathrm{V}, 50 \%$ duty cycle and in a frequency range of $15-25 \mathrm{kHz}$ are shown in Figure 27. This figure shows that the temperature profiles of the Si IGBTs with SiC Schottky diodes do not change much with switching frequency. However, the temperatures of Si IGBTs with Si diodes increase with switching frequency. This also shows that the SiC diodes have less effect on the main power switch because of the negligible reverse-recovery losses. Even though the temperatures of the Si IGBT in the all-Si converter increase as the switching frequency increases, the Si pn diode compared to the SiC Schottky diode still operates at a slightly lower temperature. 


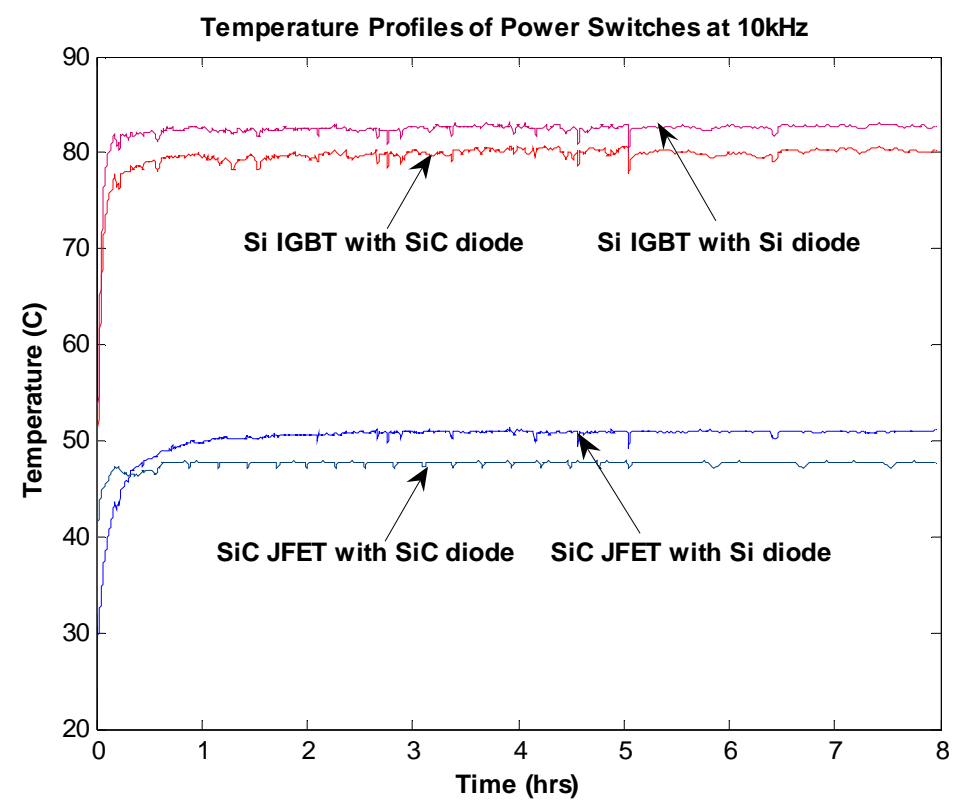

(a) Switches

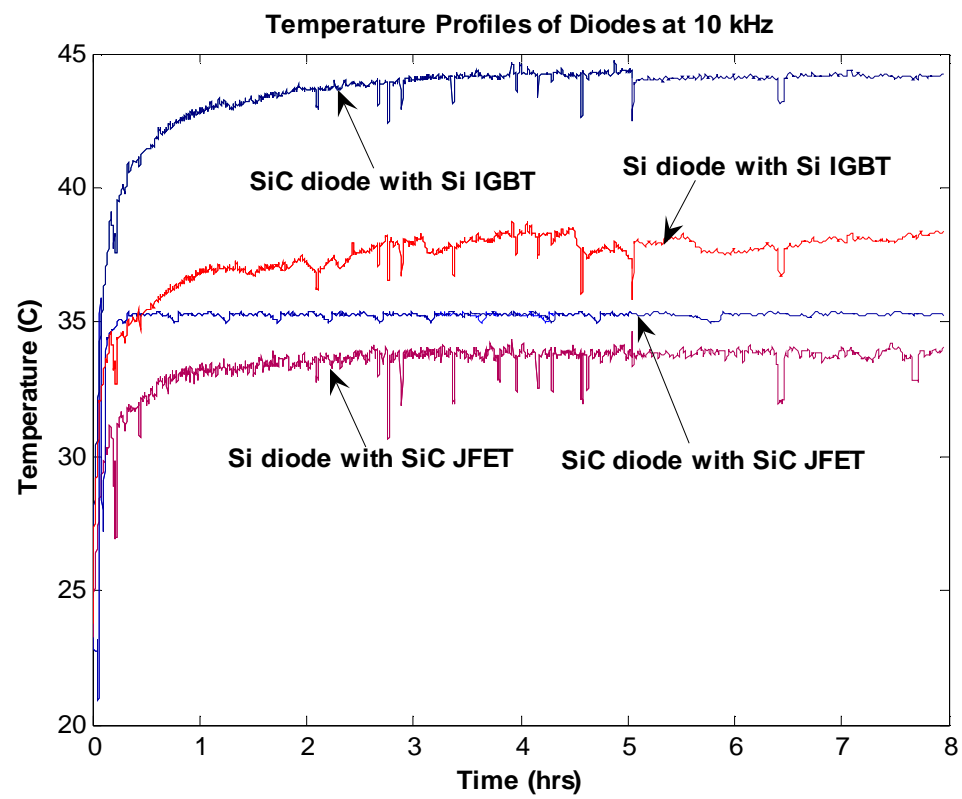

(b) Diodes

Figure 23. Temperature profile for $10-\mathrm{kHz}$ operation. 


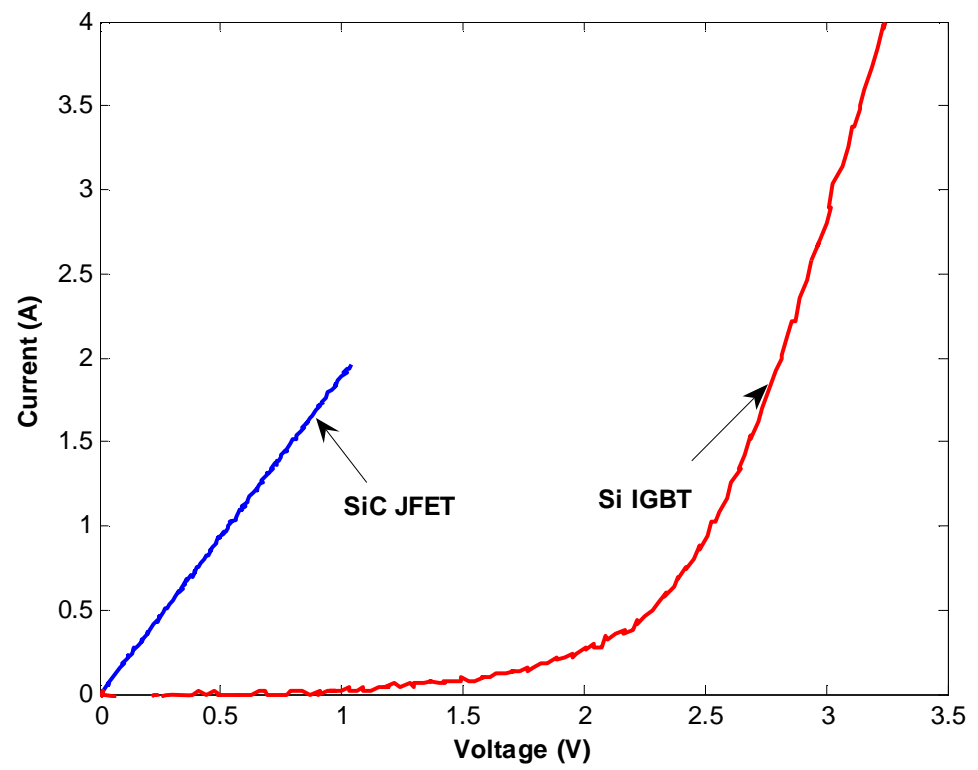

Figure 24. Comparison of static characteristics for Si IGBT and SiC JFET.

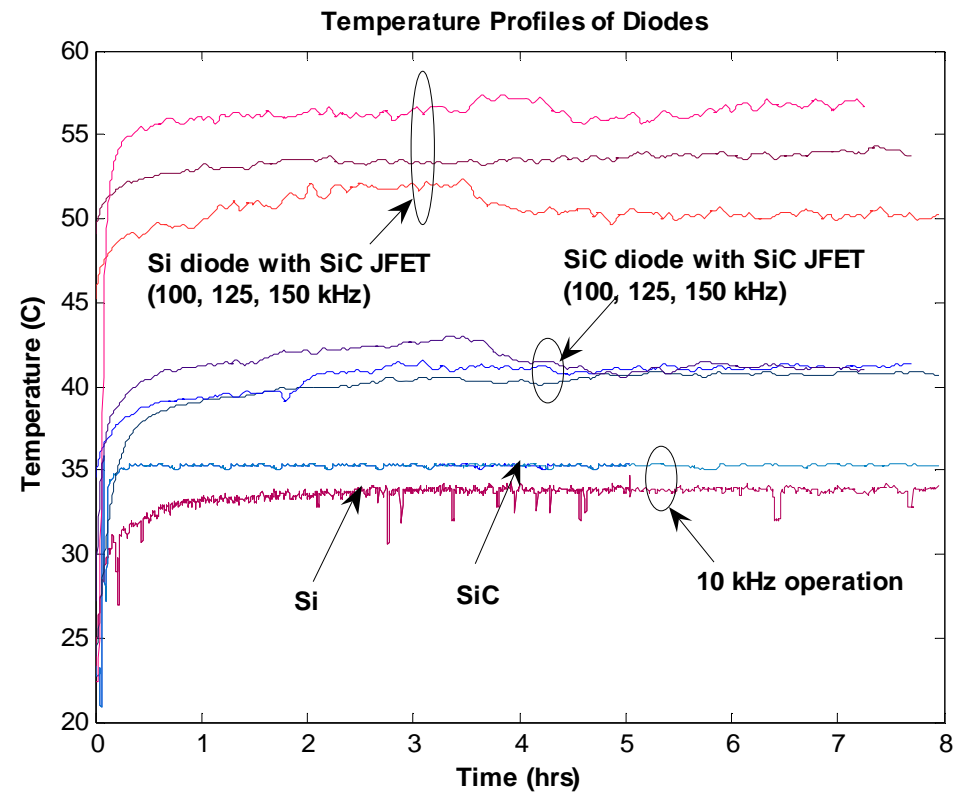

Figure 25. Temperature profile for diodes at different operating frequencies. 


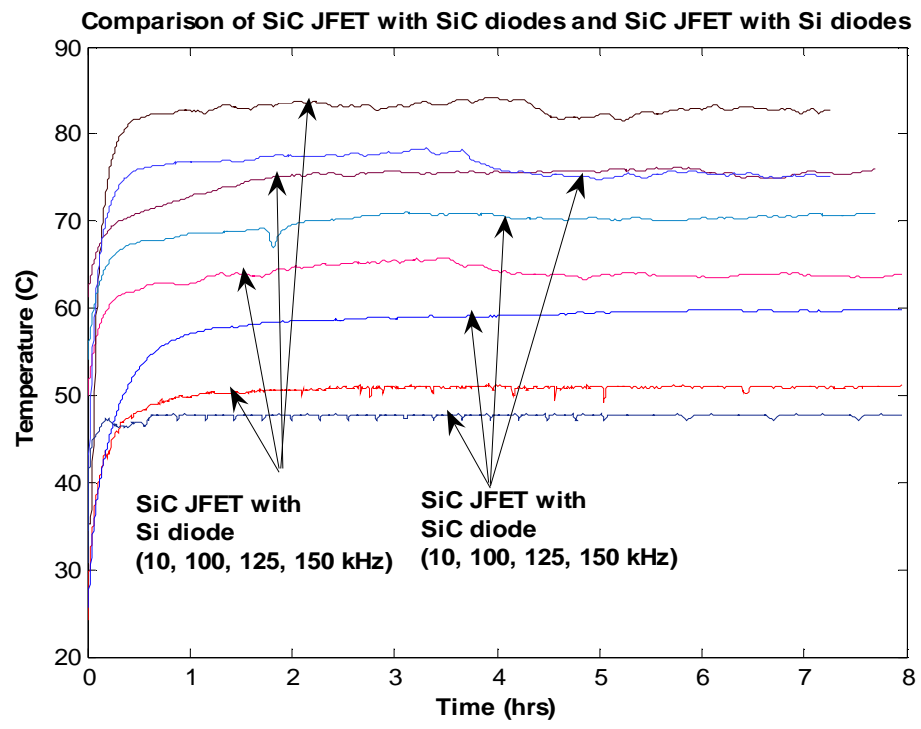

Figure 26. Temperature profile for switches at different operating frequencies.

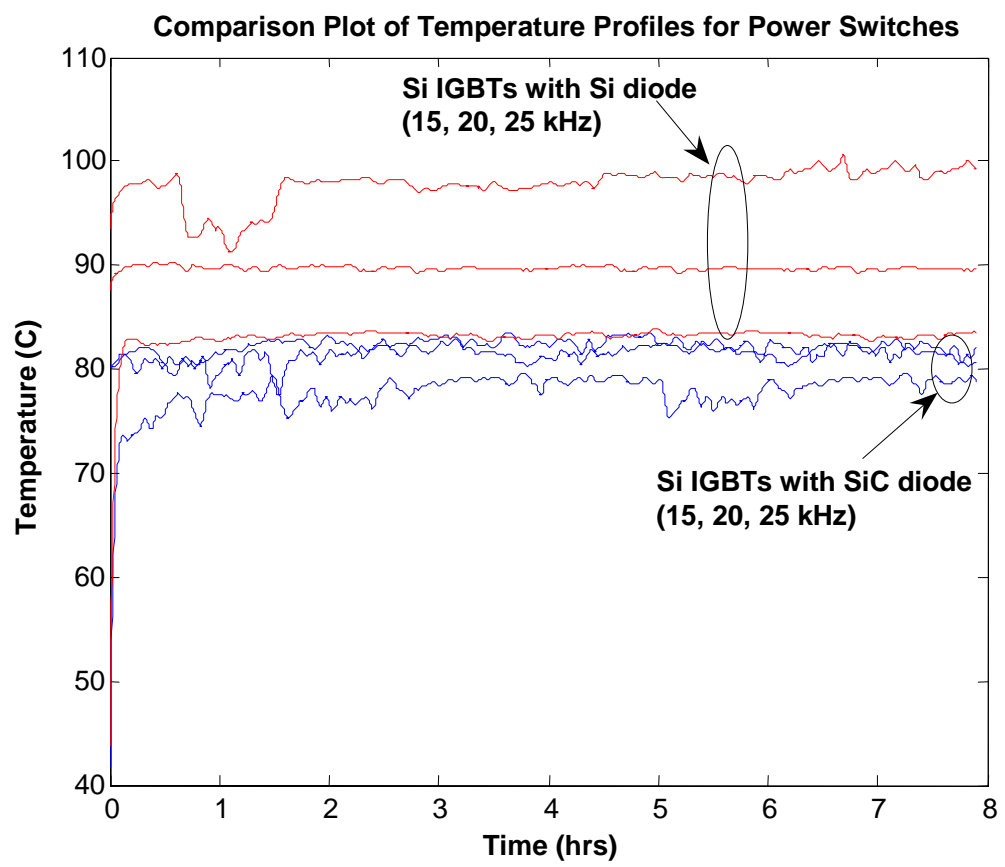

Figure 27. Plot of temperature profile for power switches at different frequencies

During the tests, several power switches failed at different operating conditions. The diodes never failed over several months of operation. Si IGBTs failed at certain operating conditions because of the thermal limits of the devices. Only case temperatures were measured; the junction temperatures would be much higher. Also, the devices were operated without any snubber circuit, thus the power switches were stressed even more than under normal operation.

SiC JFETs operated without any failures even at higher voltage levels and without heat sinks. However, the JFETs failed repeatedly after several days of operation. After investigating the devices that 
failed, it was found that gate-to-source of some devices were shorted while some had open drain-tosource failures. This shows that these experimental samples need more reliability improvement.

\section{Conclusion}

With the present SiC Schottky diode technology, when SiC Schottky diodes are used in traction drive inverters instead of Si pn diodes, the average losses of the inverter can be reduced by up to $33.6 \%$. The cost issue still remains to be a problem.

The 7.5-kW all-SiC inverter and buck converter tests showed that more research is required on $\mathrm{SiC}$ JFETs to improve their characteristics and reliability. Even though the reliability of SiC power switches is of concern, their performance is still promising. It is important to follow the present developments in $\mathrm{SiC}$ power-device technology closely because the pace of improvement has increased considerably. Better, less expensive, and more reliable $\mathrm{SiC}$ power devices are not too far away.

\section{Future Direction}

FY 2006-2007: Conduct research in high-temperature packaging for $\mathrm{SiC}$ power devices, as present device packaging cannot handle the temperatures at which $\mathrm{SiC}$ devices are capable of operating. External collaboration will be required. Conduct a feasibility study to build a $55-\mathrm{kW}$ all-SiC inverter by using the device and system-level simulation models developed based on the results from the inverter tests performed in FY05.

FY 2007-2008: Provided that the feasibility study shows that the $\mathrm{SiC}$ technology is ready for a 55$\mathrm{kW}$ all-SiC inverter, build this inverter and compare it to an all-Si inverter at full scale.

FY 2006-2008: Continue acquiring, testing, characterizing, and modeling new $\mathrm{SiC}$ devices.

\section{$\underline{\text { Publications }}$}

M. Chinthavali, L. M. Tolbert, B. Ozpineci, "4H-SiC GTO Thyristor Loss Model for HVDC Converter," pp. 1238-1243 in IEEE Industry Applications Society Annual Meeting, October 3-7, 2004, Seattle, Washington.

M. Chinthavali, B. Ozpineci, L. M. Tolbert, "High Temperature Characterization of SiC Power Electronic Devices," pp. 43-47 in IEEE Workshop on Power Electronics in Transportation, October 2122, 2004, Dearborn, Michigan.

M. Chinthavali, B. Ozpineci, L. M. Tolbert, "Enhancing Power Electronic Devices with Wide Bandgap Semiconductors," International Journal of High Speed Electronics, in press.

M. Chinthavali, B. Ozpineci, L. M. Tolbert, "High Temperature and High Frequency Performance Evaluation of 4H-SiC VJFET and Schottky Diodes," IEEE Applied Power Electronics Conference, March 6-10, 2005, Austin, Texas.

B. Ozpineci, M. Chinthavali, L. M. Tolbert, “A $55 \mathrm{~kW}$ Three-Phase Automotive Traction Inverter with SiC Schottky Diodes," IEEE Vehicle Power and Propulsion (VPP) Conference, September 7-9, 2005, Illinois Institute of Technology, Chicago, IL.

M. Chinthavali, B. Ozpineci, L. M. Tolbert, A. S. Kashyap, Wide Bandgap Electronics, ORNL TM report, October 2005.

\section{References}

1. B. Ozpineci, L. M. Tolbert, S. K. Islam, and F. Z. Peng, "Testing, Characterization, and Modeling of SiC Diodes for Transportation Applications," pp. 1673-1678 in IEEE Power Electronics Specialists Conference, Cairns, Australia, June 23-27, 2002.

2. L. M. Tolbert, B. Ozpineci, S. K. Islam, and F. Z. Peng, "Impact of SiC Power Electronic Devices for Hybrid Electric Vehicles," pp. 765-771 in SAE 2002 Transactions Journal of Passenger CarsElectronic and Electrical Systems, ISBN 0-7680-1291-0, 2003. 
3. M. Ruff, H. Mitlehner, and R. Helbig, "SiC Devices: Physics and Numerical Simulation," pp. 10401054 in IEEE Transactions on Electron Devices, June 1994.

4. D. Peters, H. Mitlehner, R. Elpelt, R. Schorner, and D. Stephani, "State of the Art Technological Challenges of SiC Power MOSFETs Designed for High Blocking Voltages," European Conference on Power Electronics and Applications, Toulouse, France, September 2-4, 2003.

5. S. H. Ryu, A. Agarwal, J. Richmond, J. Palmour, N. Saks, and J. Williams, "10 A, 2.4 kV Power DIMOSFETs in 4H-SiC," pp. 321-323 in IEEE Electron Device Letters, June 2002.

6. M. Chinthavali, B. Ozpineci, and L. M. Tolbert, "Enhancing Power Electronic Devices with Wide Bandgap Semiconductors," Advanced Workshop on 'Frontiers of Electronics' (WOFE'04), Aruba, December 17-22, 2004.

7. A. S. Kashyap, "Compact Modeling of Silicon Carbide Junction Field Effect Devices," M.S. Thesis, Department of Electrical Engineering, University of Arkansas, Fayetteville, Arkansas, 2005. 


\subsection{Integrated dc/dc Converter for Multi-Voltage Bus Systems}

Principal Investigator: Gui-Jia Su

Oak Ridge National Laboratory

National Transportation Research Center

2360 Cherahala Boulevard

Knoxville, TN 37932

Voice: 865-946-1330; Fax: 865-946-1400; E-mail: sugj@ornl.gov

DOE Technology Development Manager: Susan A. Rogers

Voice: 202-586-8997; Fax: 202-586-1600; E-mail: Susan.Rogers@ee.doe.gov

ORNL Program Manager: Mitch Olszewski

Voice: 865-946-1350; Fax: 865-946-1262; E-mail: olszewskim@ornl.gov

\section{Objectives}

- Develop integrated dc/dc converters for triple-voltage busses (14-V/42-V/high-voltage) to reduce component count, size, and cost.

- Produce converter prototypes and testing data that can demonstrate the converter's capability for bidirectional power flow control among the three busses and can be useful in setting FreedomCAR technical targets for $\mathrm{dc} / \mathrm{dc}$ converters.

\section{Approach}

The following approaches are taken to reduce the cost and size, and to increase the efficiency and power density, of dc/dc converters for multi-bus systems in hybrid electric vehicles (HEVs) and fuel cellpowered vehicles (FCVs).

- Minimize the number of switches and thus the associated gate drivers to reduce the components count and cost.

- Employ soft switching and synchronous rectification to increase efficiency and power density.

- Employ soft switching to lower electromagnetic interference (EMI) noise.

- Refine the topology to further reduce the components count.

- Use flexible topology—same circuit applicable to both dual- and triple-voltage systems.

\section{Major Accomplishments}

- A half-bridge-based dc/dc converter was developed that can interconnect the three voltage busses and can reduce component count by $50 \%$ compared with conventional technologies. A novel control scheme was also devised that can control the power flow among the three busses.

- For hardware demonstration and to produce test data for cost and performance evaluation, a 2-kW converter prototype was designed and built.

- Testing of the converter was successfully completed and a conversion efficiency of $92 \%$ was achieved over a wide load range.

- To further reduce the component count, a reduced-parts converter was designed and fabricated. Initial testing data have proved the concept and indicated it is a promising alternative topology. Further testing will be conducted to gather performance data. 


\section{$\underline{\text { Technical Discussion }}$}

\section{Introduction}

The 14-V electrical system in current automobiles has reached its capability limits and cannot meet the demands of future electrical loads. As HEVs and FCVs emerge, there is an increasing desire to replace more engine-driven mechanical and hydraulic systems with electrical systems to increase efficiency. Presently, 42-V systems are being proposed to cope with the increasing electrical loads. During the transition to the $42-\mathrm{V}$ system, most automobiles are expected to employ a $14-\mathrm{V} / 42-\mathrm{V}$ duallevel voltage system, in which a bi-directional dc/dc converter is required to connect the two voltage networks. Additionally, most battery-powered HEVs employ a high-voltage (200 500-V) bus for the traction motor drive. In HEVs with a 42-V alternator, a dc/dc converter supplied from the 42-V bus may be used to charge the high-voltage battery, as shown in Figure 1(a). On the other hand, for HEVs having a generator directly connected on the high-voltage bus, a dc/dc converter is typically required to charge the $14-\mathrm{V}$ and/or $42-\mathrm{V}$ batteries.

Furthermore, FCVs will require a bi-directional dc/dc converter to interconnect the fuel cell-powered high-voltage bus and the low-voltage busses for vehicle auxiliary loads. For the reasons mentioned earlier, both the $14-\mathrm{V}$ and $42-\mathrm{V}$ busses may be employed to power vehicle accessory loads, as shown in Figure 1(b). An energy storage device is also required for fuel cell start-up and for storing the energy captured by regenerative braking, because the fuel cells lack energy storage capability. One way to accomplish this is to use the vehicle $14-\mathrm{V}$ or $42-\mathrm{V}$ battery with the bi-directional dc/dc converter. During vehicle starting, the high-voltage bus is boosted up to around $300 \mathrm{~V}$ by the dc-dc converter drawing

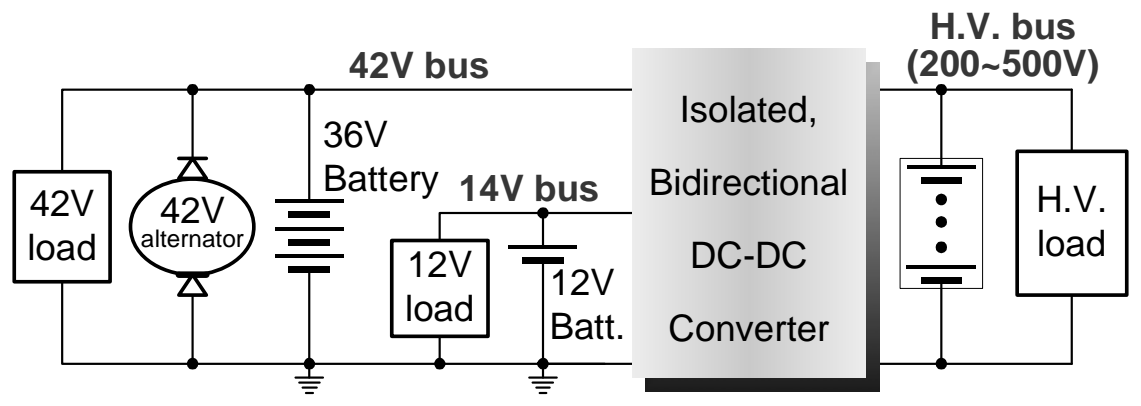

(a) Hybrid electric vehicles

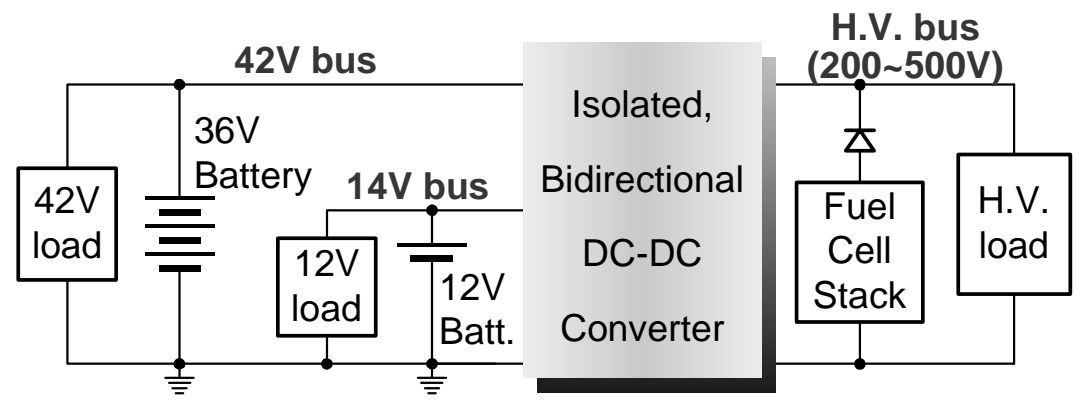

(b) Fuel cell-powered vehicles

Figure 1. A dc/dc converter interconnecting 14-V/42-V/high-voltage bus nets in HEVs and FCVs. 
power from the $14-\mathrm{V}$ or $42-\mathrm{V}$ battery. This high-voltage bus then supplies power for the fuel cell compressor motor expanding unit controller and brings up the fuel cell voltage, which in turn feeds back to the high-voltage bus to release the loading from the battery.

In summary, a triple-voltage bus system (14-V/42-V/high-voltage) will likely be employed in HEVs and FCVs, as shown in Figure 1, before all vehicle auxiliary electrical components are moved to the 42-V bus. While dc/dc converters are available to interconnect any two of the busses, to reduce component count, size, cost, and volume, it is desirable to employ an integrated $\mathrm{dc} / \mathrm{dc}$ converter to interconnect the three voltage busses instead of using two separate converters. Aside from bi-directional power control capability, the converter needs to provide galvanic isolation between the low- and high-voltage busses to meet safety requirements. Further, soft switching is preferred over hard switching because it offers a reduced level of EMI and switching losses.

In this project, a low-cost, soft-switched, isolated bi-directional dc/dc converter is being developed for interconnecting the three bus nets. The converter is based on a dual half-bridge topology and uses snubber capacitors and the transformer leakage inductance to achieve zero-voltage switching (ZVS). Therefore, no extra resonant components are required for ZVS, further reducing the component count. The inherent soft-switching capability and the low component count of the converter allow high powerdensity, efficient power conversion, and compact packaging. A novel power flow control scheme based on a combined duty ratio and phase shift angle control was also devised. A 2-kW prototype was designed, built, and tested to produce hardware demonstration and testing data that will be useful in setting FreedomCAR technical targets for dc/dc converters.

\section{dc/dc Converter for Triple Voltage Busses}

\section{Description of the dc/dc converter}

Figure 2 shows a schematic of the dc/dc converter, which consists mainly of dual half-bridge inverters/converters and a high-frequency transformer, $T r$. The high-frequency transformer provides the required galvanic isolation and voltage level matching between the $14-\mathrm{V}$ and $42-\mathrm{V}$ busses and the highvoltage bus, while the $14-\mathrm{V}$ and $42-\mathrm{V}$ busses share a common ground. The leakage inductance of the transformer is used as the intermediate energy storing and transferring element between the two lowvoltage busses and the high-voltage net. The snubber capacitors, $\mathrm{C}_{\mathrm{r} 1} \sim \mathrm{C}_{\mathrm{r} 4}$, resonate with the transformer leakage inductance to provide ZVS.

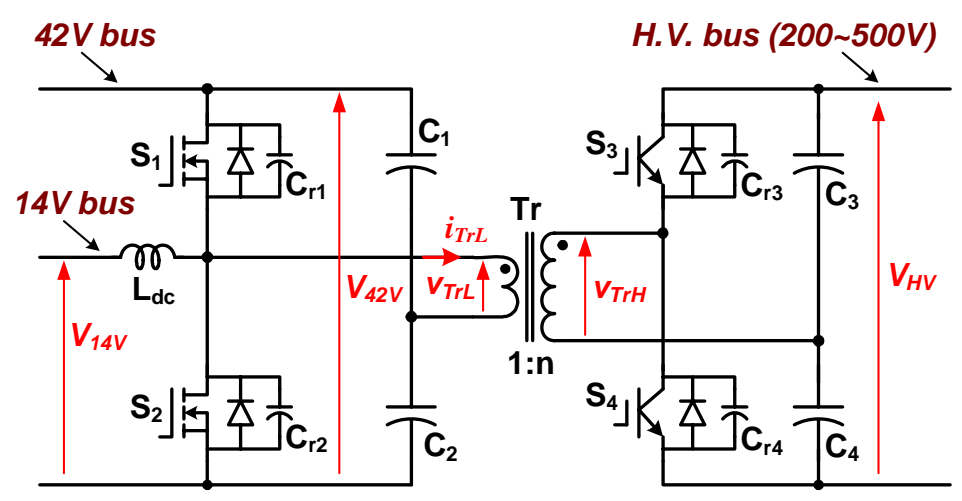

Figure 2. Schematic of the soft-switched bi-directional dc/dc converter.

Duty ratio control is used for power flow control between the $14-\mathrm{V}$ and $42-\mathrm{V}$ busses, making the two bus voltages, $V_{14 V}$ and $V_{42 V}$, track to each other by $V_{14 V}=d \bullet V_{42 V}$, where $d$ is the duty ratio of the switches $\mathrm{S}_{1}$ and $\mathrm{S}_{3}$. For $14-\mathrm{V} / 42-\mathrm{V}$ systems, the duty ration is fixed at $d=1 / 3$ for normal operation and can be changed 
to adjust the state of charge of the low-voltage batteries if necessary. In addition, a phase shift angle, $\phi$, between the transformer primary and secondary voltages is employed for power flow control between the $42-\mathrm{V}$ and high-voltage busses as shown in Figure 3, where $d=1 / 3$. The power transferred through the transformer can be expressed by

$$
P=\frac{V_{42 v} V_{H V}}{n} \cdot \frac{\phi}{2 \pi f_{s w} L_{s}} \cdot\left[\frac{2}{9}-\frac{\phi}{4 \pi}\right]
$$

where $n$ is transformer turns ratio, $L s$ is transformer leakage inductance, and $f s w$ is switching frequency.

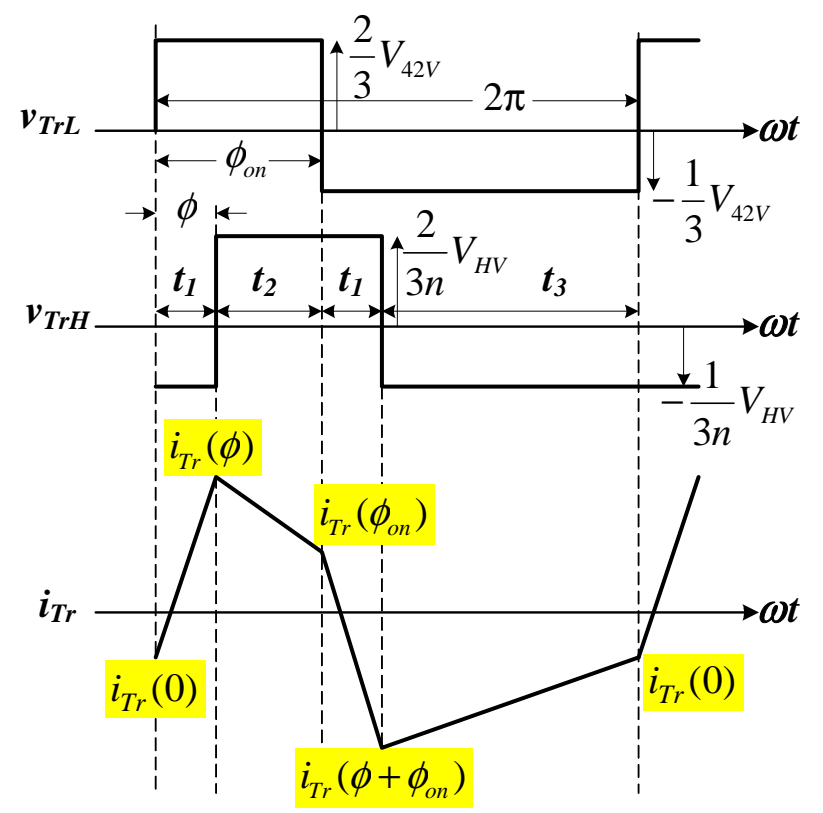

Figure 3. Ideal transformer voltage and current waveforms illustrating power flow control between the $42-\mathrm{V}$ and high-voltage busses at $d=1 / 3$.

For a given design, the maximum power is determined by

$$
P_{\max }=\frac{V_{42 v} V_{H V}}{n} \cdot \frac{2}{81 f_{s w} L_{s}} \text { at } \phi_{P_{\max }}=\frac{4 \pi}{9} .
$$

Figure 4 plots the power versus the phase shift angle at different voltage levels of $V_{H V}$ with $n=8$, $L s=0.16 \mu \mathrm{H}, f s w=40 \mathrm{kHz}$, and $V_{42 v}=42 \mathrm{~V}$ for a prototype design. 


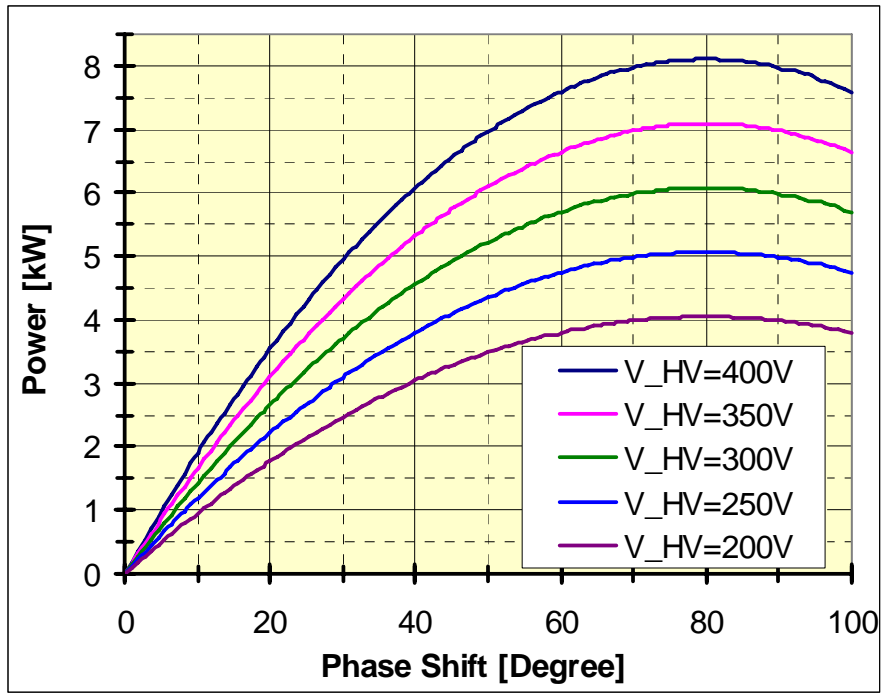

Figure 4. Power v.s. phase shift angle at various levels of $\mathrm{V}_{\mathrm{HV}}\left(n=8, L s=0.16 \mu \mathrm{H}, f s w=40 \mathrm{kHz}, V_{42 v}=42 \mathrm{~V}\right)$.

\section{Simulation and Experimental Results}

A 2-kW prototype was designed, built, and tested to verify the power flow control scheme and to evaluate its performance. Specifications of the prototype are listed below.

- Rating: $2 \mathrm{~kW}$ continous $/ 5 \mathrm{~kW}$ maximum

- Efficiency: $>92 \%$ over a wide load range

A detailed circuit simulation was first performed to verify the design goal, which is to guarantee at least $2 \mathrm{~kW}$ of power can be transferred through the transformer at a voltage range of 200 400V for the high-voltage bus. Figure 5 shows simulation results where power is transferred from the low-voltage busses to the high-voltage net, in (a) at $\mathrm{V}_{\mathrm{HV}}=200 \mathrm{~V}, 2.1 \mathrm{~kW}$ is provided to the high-voltage bus, while in (b) at $\mathrm{V}_{\mathrm{HV}}=400 \mathrm{~V}$, power is increased to $5.1 \mathrm{~kW}$. Figure 6 shows simulated waveforms when the power flow is reversed. The simulation results confirm the design goal of the power ratings.

A 2-kW prototype was then designed and built based on the analytical, simulation, and preliminary proof-of-concept testing results. Figure 7 shows a photo of the prototype, which is laid on a 0.375 -in. liquid-cooled heat sink with a footprint $7.5 \mathrm{in}$. wide by $13.5 \mathrm{in}$. long. It was intentionally laid loosely to provide spaces for easy probing access.

The high-voltage switches are implemented with two CoolMOS metal oxide semiconductor fieldeffect transistors (MOSFETs made by IXYS, which are rated at 600V/50A with an on-resistance of 35 $\mathrm{m} \Omega$. High-speed insulated-gate bipolar transistors (IGBTs) will also be tested. The low-voltage switches are MOSFETs made by International Rectifier, rated at 75V/90A with an on-resistance of $4.5 \mathrm{~m} \Omega$.

The inductor was fabricated using a Metglas ${ }^{\mathrm{TM}}$ amorphous,C-core (AMCC-32) with the following specifications:

- Winding: 16-mil-thick copper foil

- Inductance: $10 \mu \mathrm{H}$

- Current rating: $100 \mathrm{~A}$

The high-frequency transformer was fabricated using Ferroxcube E-cores, E65/32/27. Two different grades of soft ferrite, 3F3 and 3C94, will be tested for the transformer core. The primary winding uses a 10-mil-thick copper foil and has two turns, while the secondary winding uses 3 litz wires of 16 AWG in parallel and has 16 turns. The two windings are interleaved to minimize the leakage inductance. 


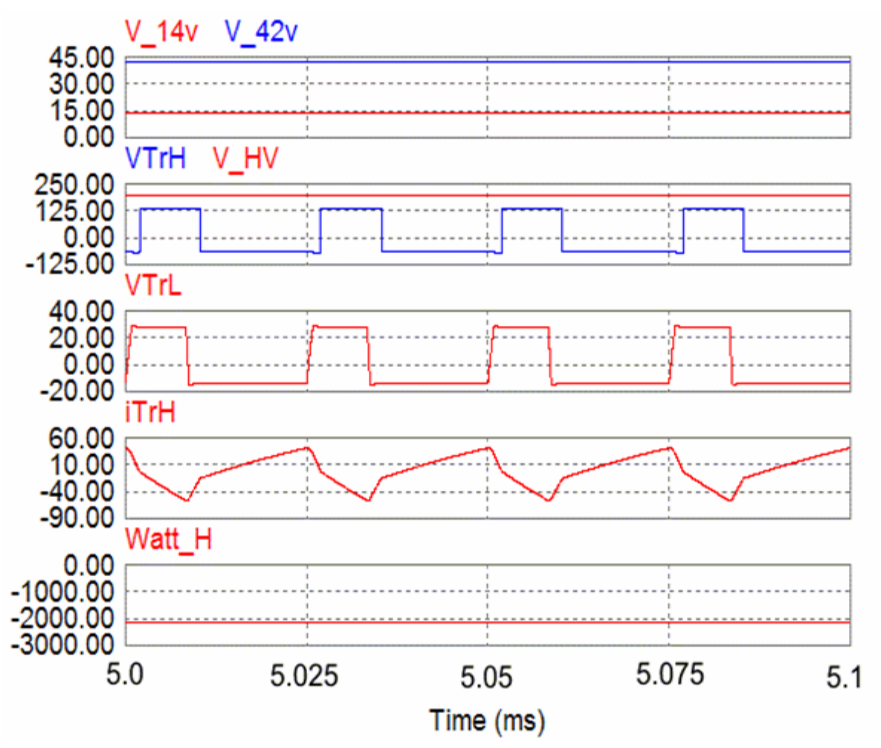

(a) $\mathrm{V}_{\mathrm{HV}}=200 \mathrm{~V}$, power transferred to the high-voltage bus: $2.1 \mathrm{~kW}$

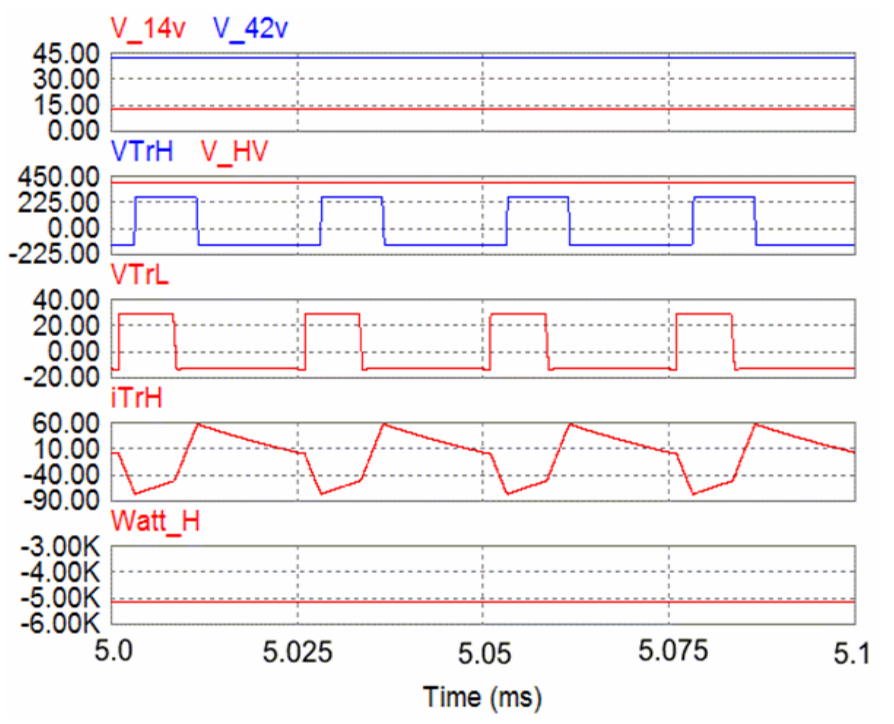

(b) $\mathrm{V}_{\mathrm{HV}}=400 \mathrm{~V}$, power transferred to the high-voltage bus: $5.1 \mathrm{~kW}$

Figure 5. Simulation results showing power is transferred from the low-voltage busses to the high-voltage net. 


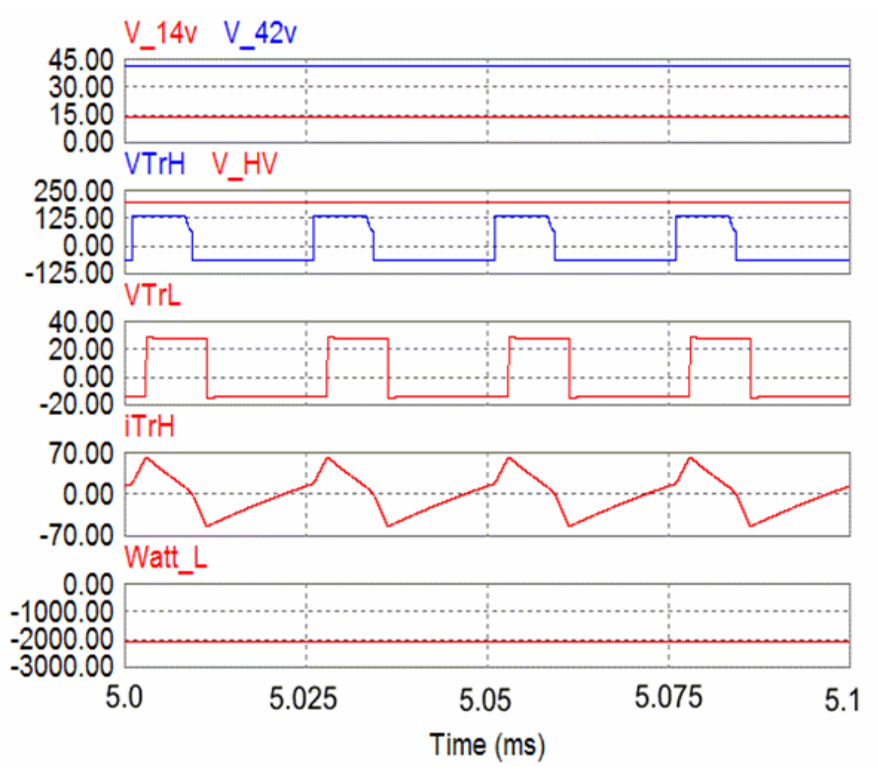

(a) $\mathrm{V}_{\mathrm{HV}}=200 \mathrm{~V}$, power transferred to the low-voltage busses: $2.0 \mathrm{~kW}$

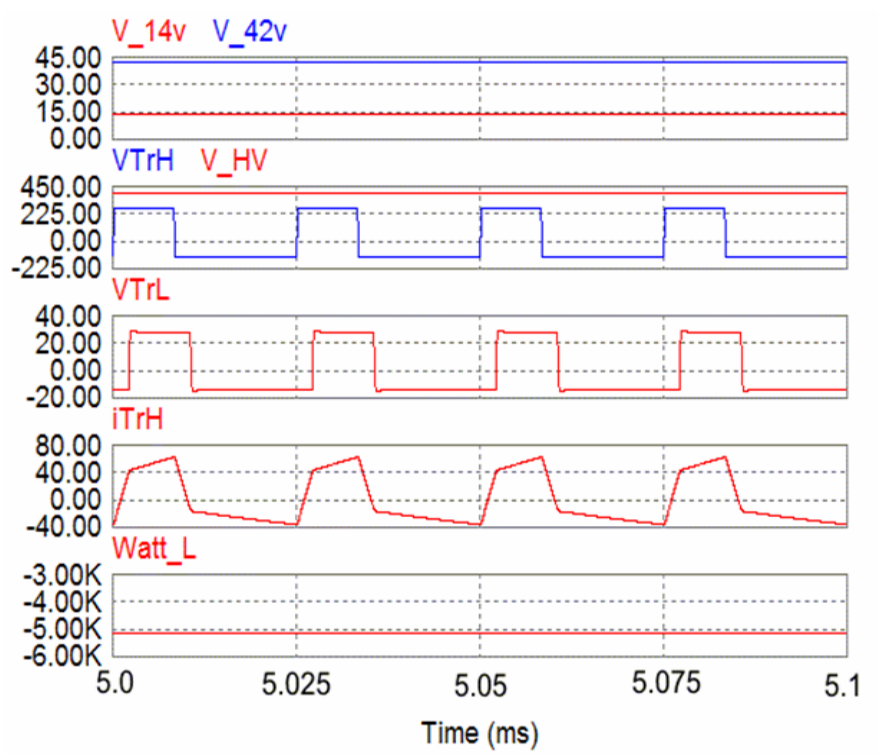

(b) $\mathrm{V}_{\mathrm{HV}}=400 \mathrm{~V}$, power transferred to the low voltage busses: $5.1 \mathrm{~kW}$

Figure 6. Simulation results illustrating power is transferred from the high-voltage net to the low-voltage busses. 


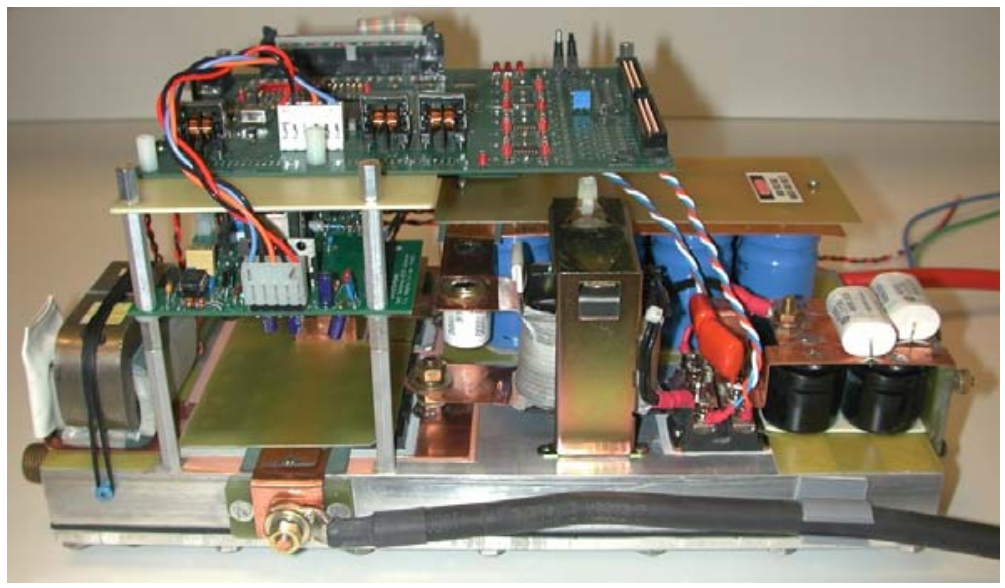

Figure 7. Photo of the prototype.

Figures 8, 9, and 10 show typical testing waveforms when power was transferred from the 42-V bus to the 14-V and high-voltage busses, from the high-voltage bus to the $14-\mathrm{V}$ and $42-\mathrm{V}$ busses, and from the $14-\mathrm{V}$ bus to the high-voltage and $42-\mathrm{V}$ busses, respectively. Figure 11 plots power conversion efficiency against the output power, indicating efficiencies of $92 \%$ were achieved over a wide load range.

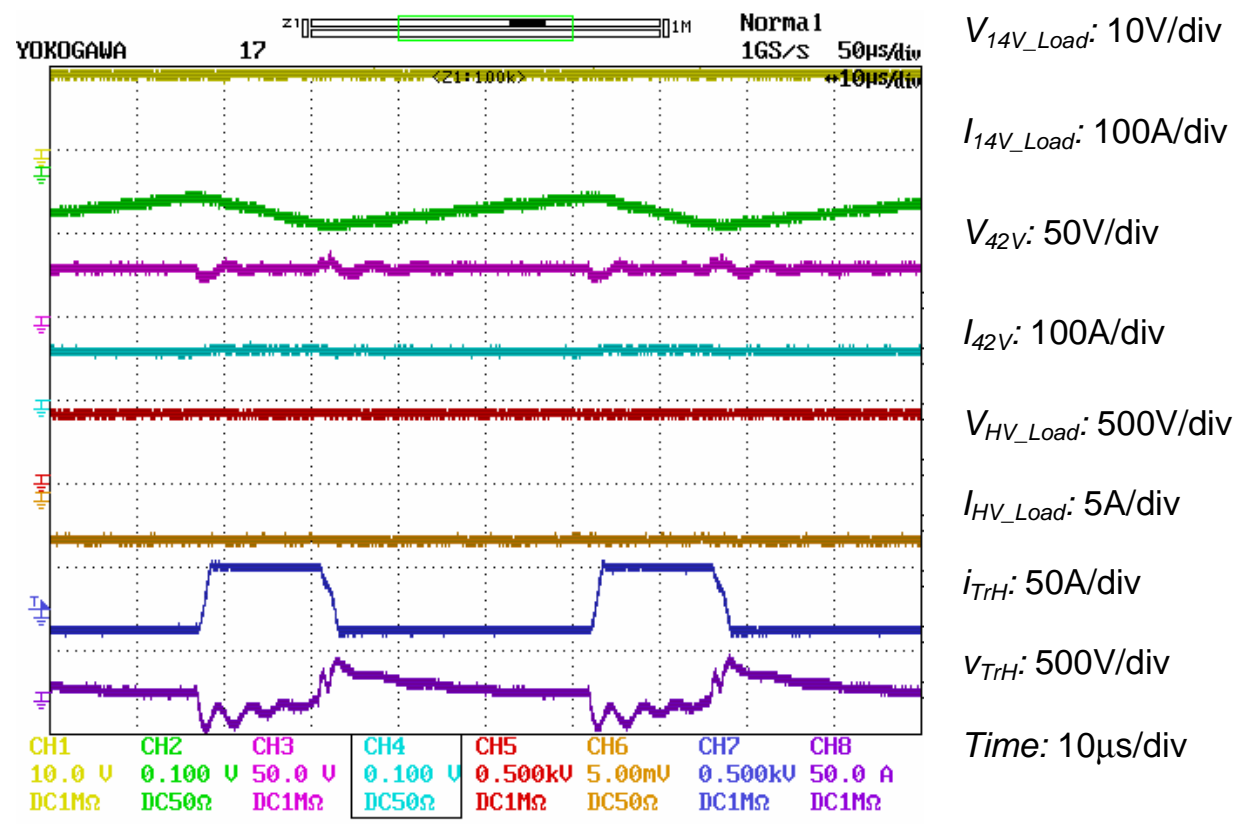

Figure 8. Typical testing waveforms for $42-\mathrm{V}$ to $14-\mathrm{V}$ and high-voltage power transfer. 


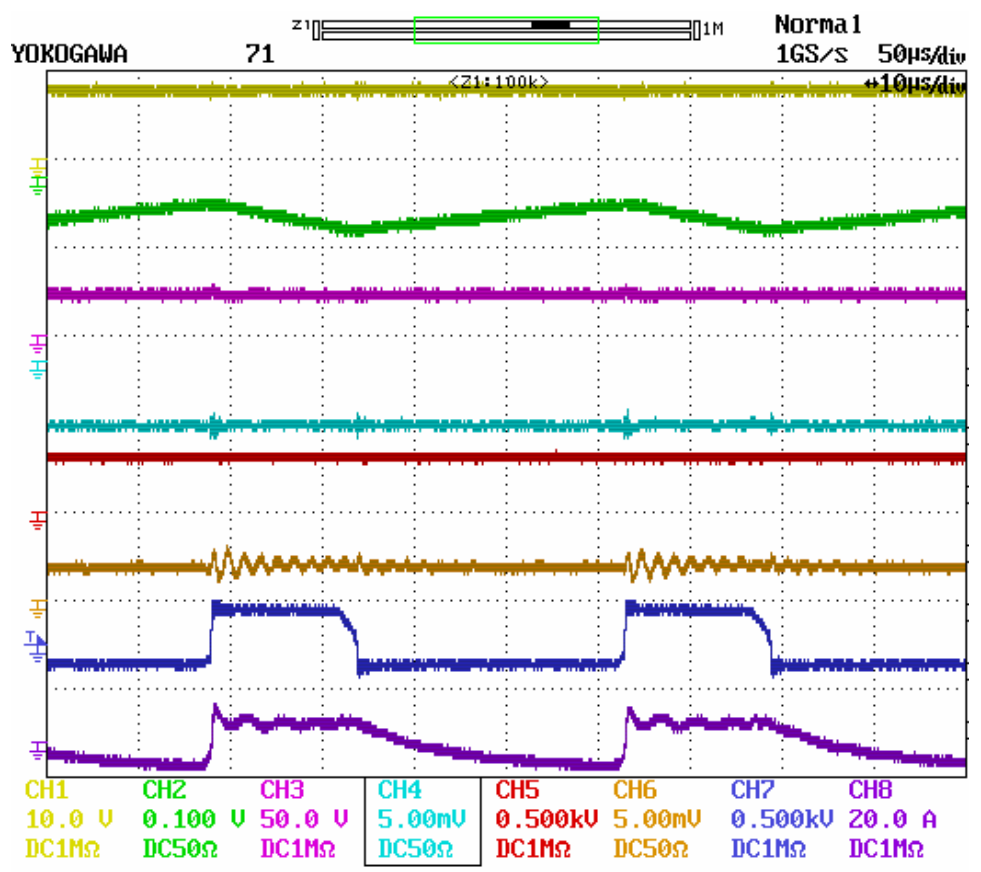

$V_{14 V_{L} \text { Load: }} 10 \mathrm{~V} / \mathrm{div}$

$I_{14 V_{\text {LLod: }}: 100 \mathrm{~A} / \mathrm{div}}$

$V_{42 V_{\text {LLad: }}: 50 \mathrm{~V} / \mathrm{div}}$

I42v_Load: $5 \mathrm{~A} / \mathrm{div}$

$V_{H V:} 500 \mathrm{~V} / \mathrm{div}$

$I_{\text {HV_Load: }}$ 5A/div

$i_{T r H}: 50 \mathrm{~A} / \mathrm{div}$

$v_{T r H}: 500 \mathrm{~V} / \mathrm{div}$

Time: $10 \mu \mathrm{s} / \mathrm{div}$

Figure 9. Typical testing waveforms for high-voltage to $14-\mathrm{V}$ and $42-\mathrm{V}$ power transfer.

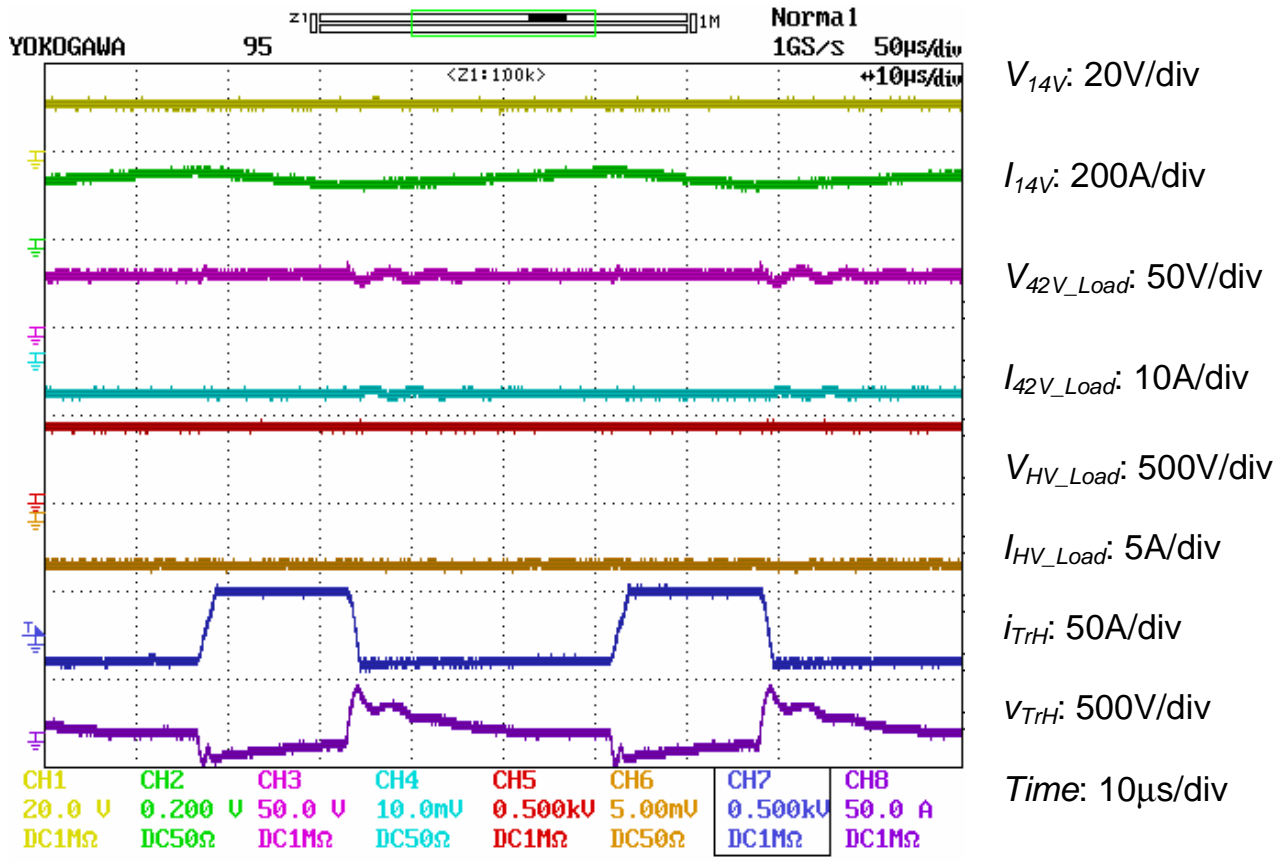

Figure 10. Typical testing waveforms for $14-\mathrm{V}$ to $42-\mathrm{V}$ and high-voltage power transfer. 


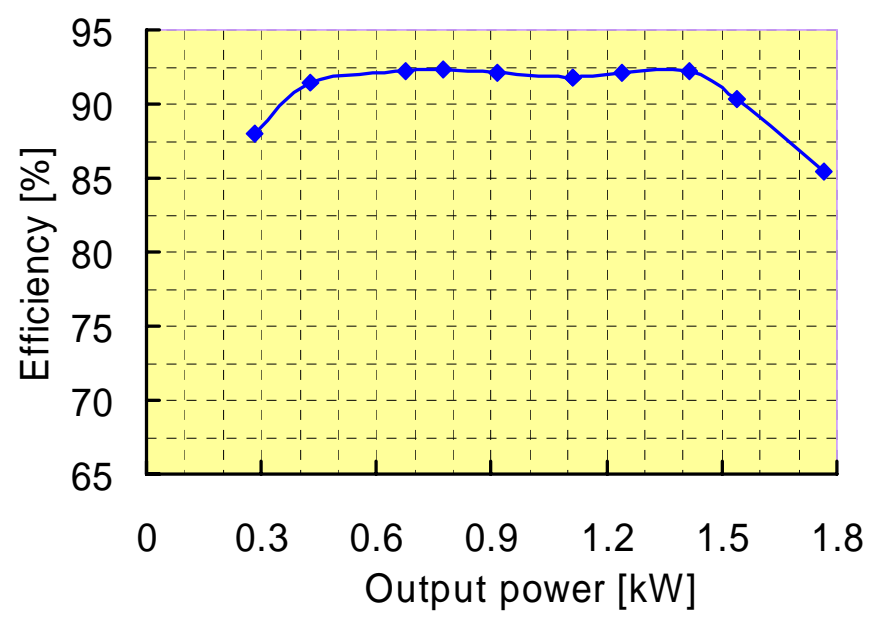

Figure 11. Efficiency chart.

\section{Reduced-Part-Count dc/dc Converter for Triple Voltage Busses}

To further reduce the component count, weight, and size of the converter, the inductor, $\mathrm{L}_{\mathrm{dc}}$ in Figure 2, which is the heaviest part except for the aluminum heat sink, has been eliminated. A 2-kW prototype was designed and fabricated. Figure 12 shows a photo of the reduced-part-count prototype. Initial testing was conducted for power transfer between the 42-V and high-voltage busses. Figures 13 and 14 show testing waveforms when power was transferred from the 42-V bus to the $14-\mathrm{V}$ and highvoltage busses at $1.1 \mathrm{~kW}$ and $1.4 \mathrm{~kW}$, respectively. Figure 15 illustrates waveforms when power was transferred from the high-voltage bus to the $14-\mathrm{V}$ and $42-\mathrm{V}$ busses at $0.54 \mathrm{~kW}$. The initial testing results clearly verified the bi-directional power flow control capability of the reduced part count version. Further testing will be conducted to collect data for cost and performance evaluations.

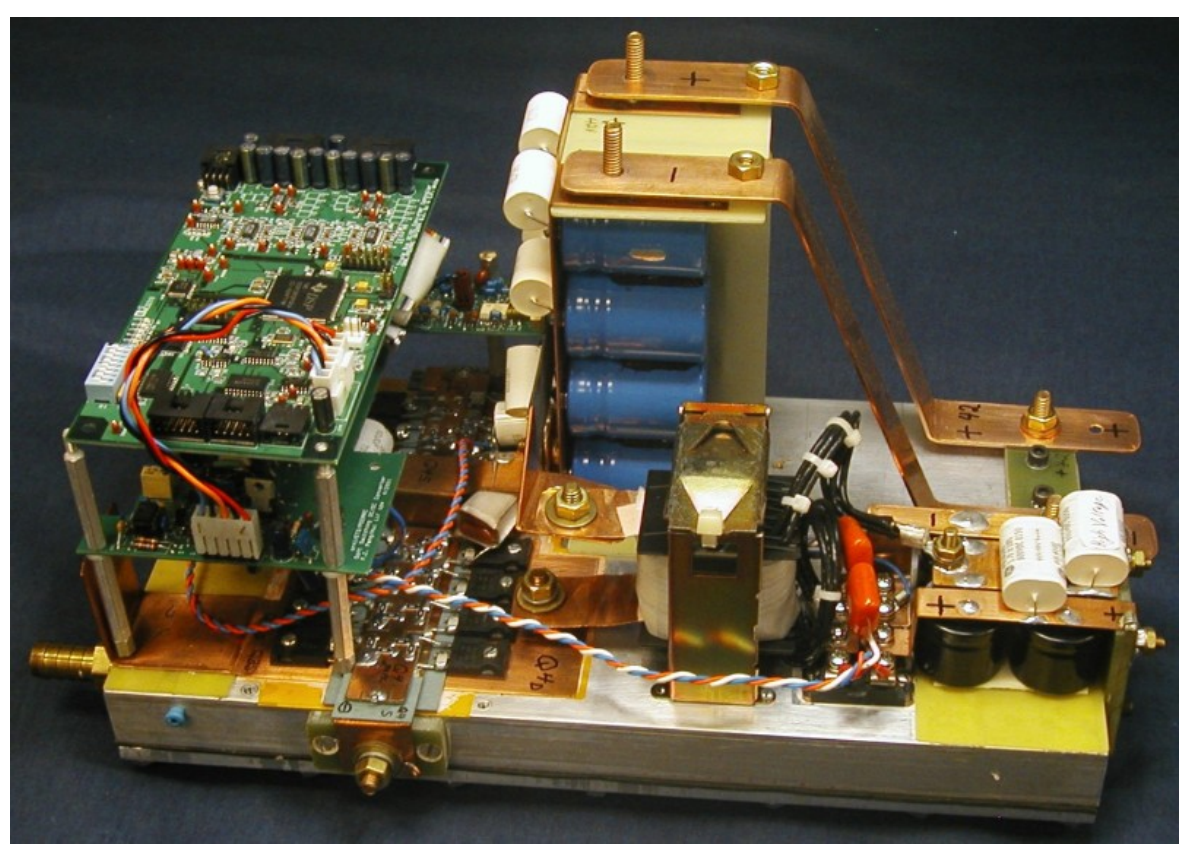

Figure 12. Photo of the reduced-part count prototype. 


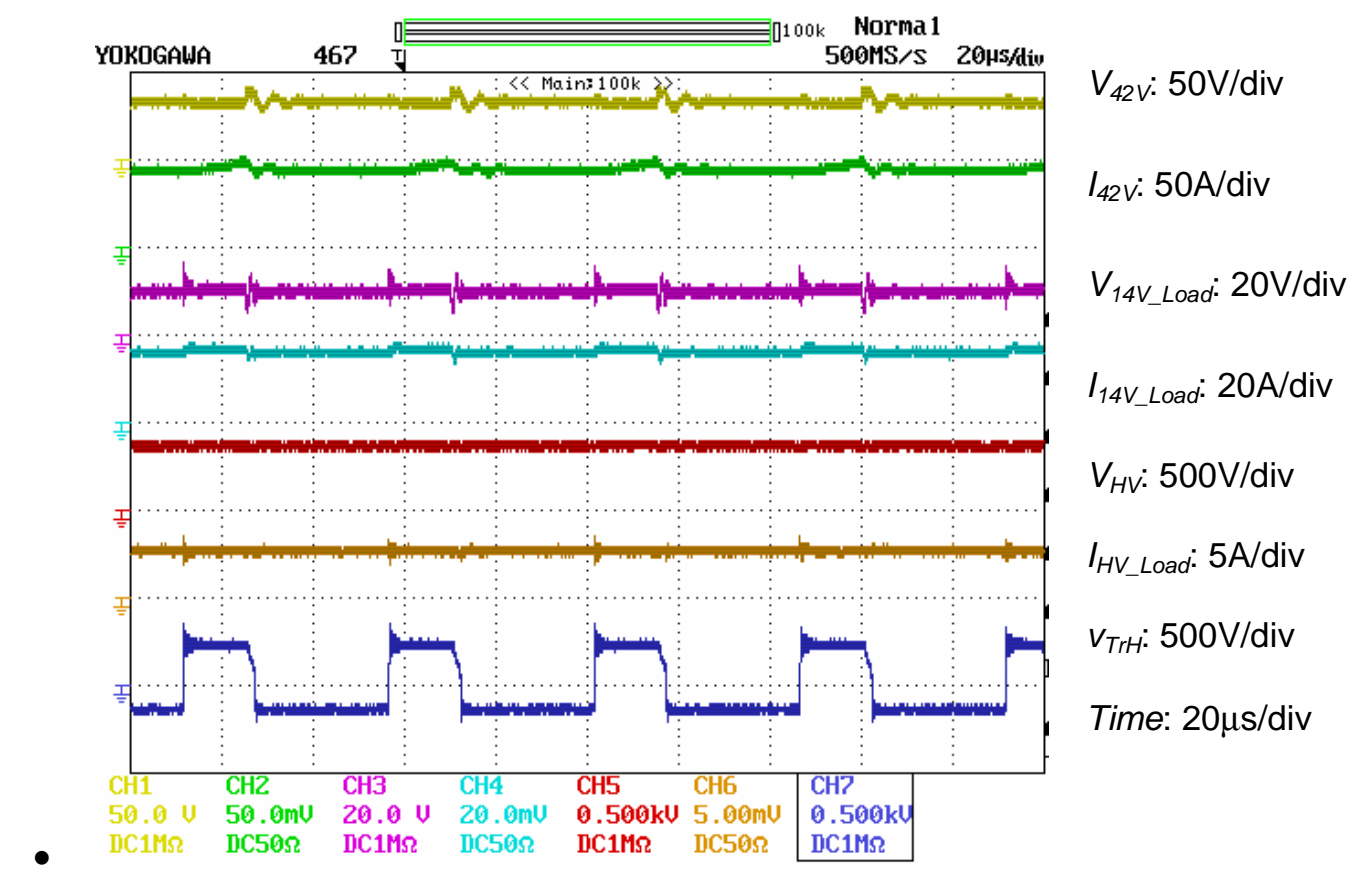

Figure 13. Testing waveforms for $42-\mathrm{V}$ to $14-\mathrm{V}$ and high-voltage power transfer at $1.1 \mathrm{~kW}$.

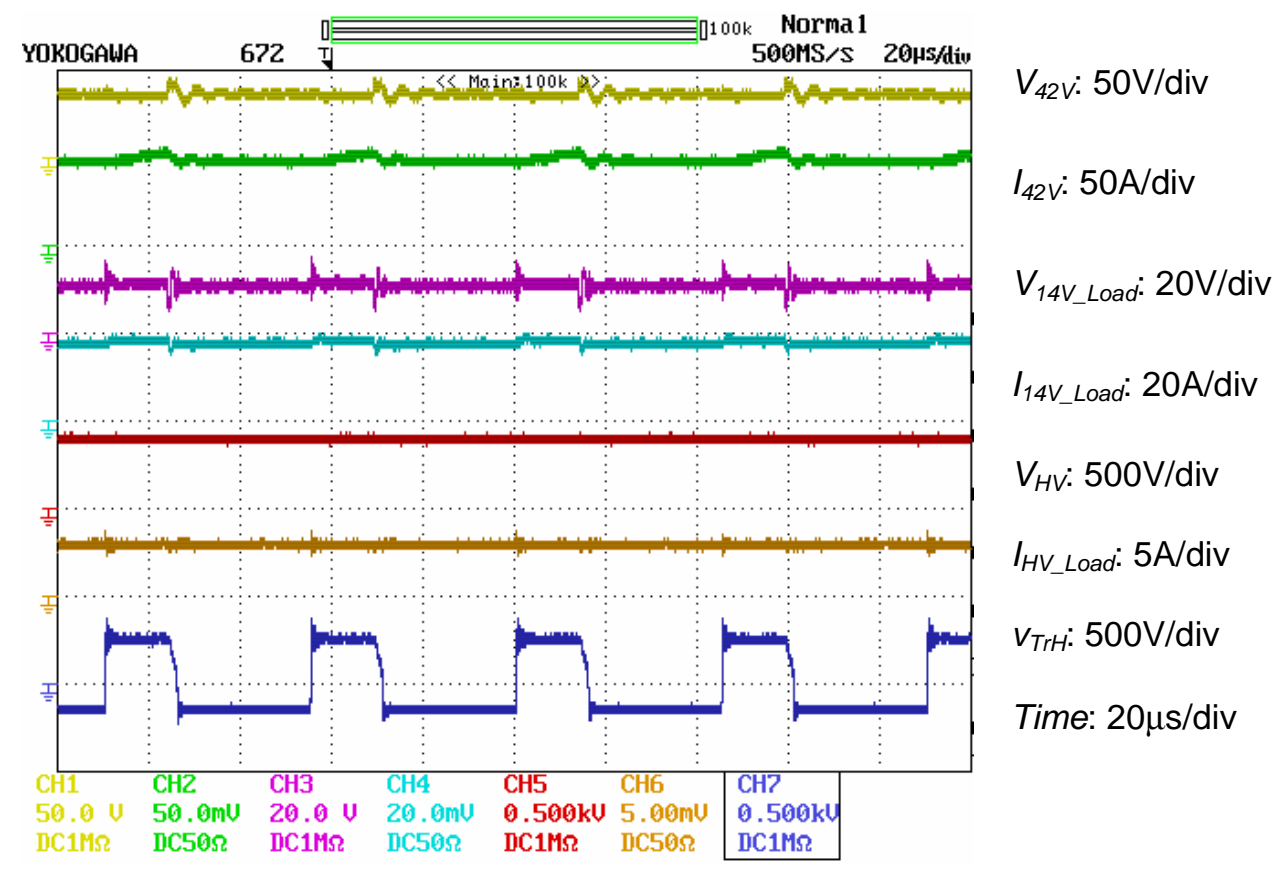

Figure 14. Testing waveforms for $42-\mathrm{V}$ to $14-\mathrm{V}$ and high-voltage power transfer at $1.4 \mathrm{~kW}$. 


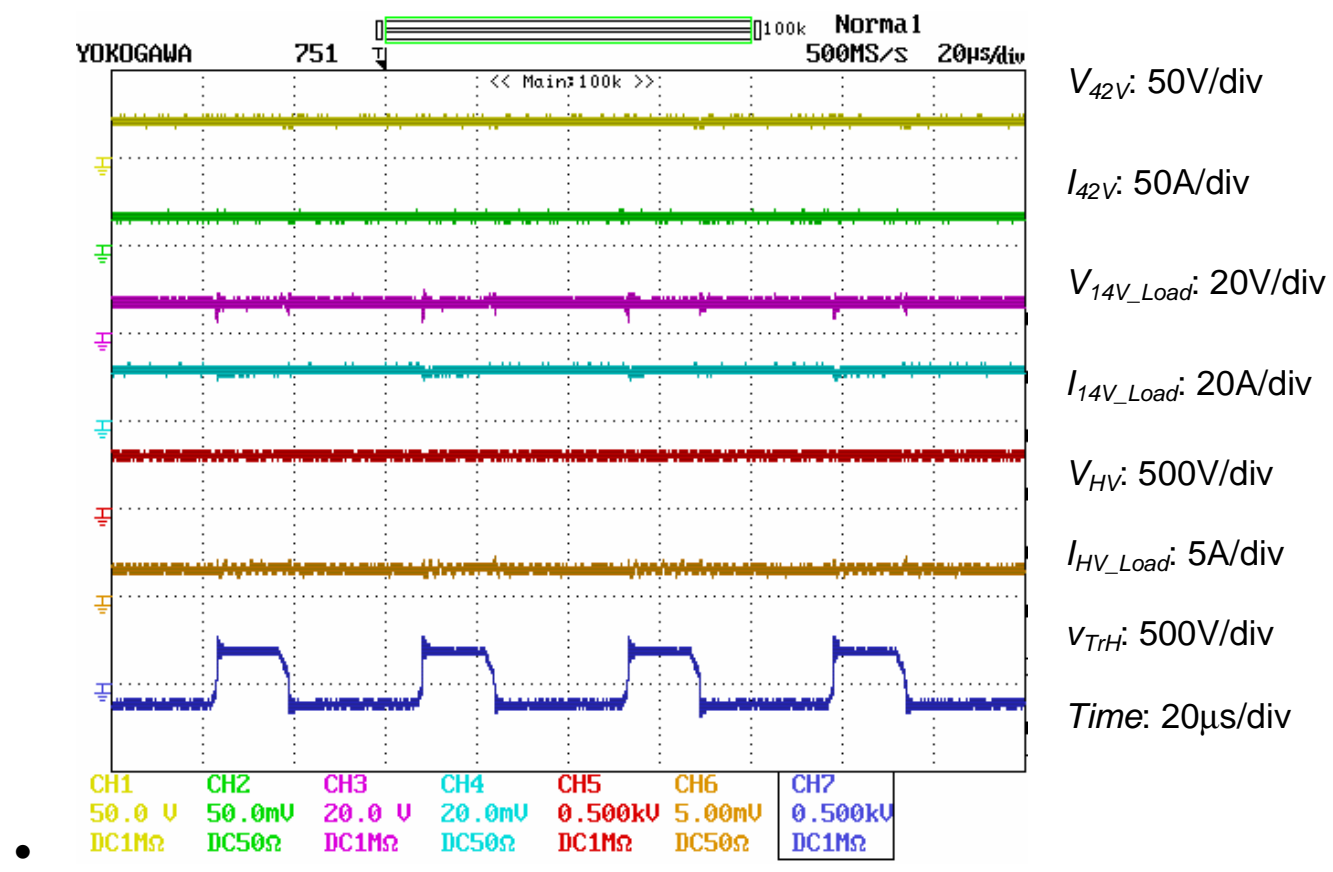

Figure 15. Testing waveforms for high-voltage to $42-\mathrm{V}$ and $14-\mathrm{V}$ power transfer at $0.54 \mathrm{~kW}$.

\section{Conclusions}

The developed integrated dc/dc converters for triple-voltage-bus (14-V/42-V/high-voltage) systems for HEV and FCV applications has the following features:

- Uses only four switching devices, leading to significant cost savings and higher power density.

- Employs soft switching and synchronous rectification, contributing to high efficiency and low EMI.

- Requires no auxiliary circuits or complex control dedicated for soft switching.

- Uses flexible power flow management as a result of the capability to transfer power among all three voltage busses by employing a combined duty ratio and phase shift angle control scheme.

Simulation and testing results on a $2-\mathrm{kW}$ prototype have confirmed the operating principles, demonstrated efficiencies of up to $92 \%$, and shown that the number of components can be reduced by 50 $\%$ over conventional technologies. The initial testing data on the reduced-part version proved the concept and indicated that it is a promising alternative to the first version. Future testing will provide data for detailed performance and cost analysis.

\section{Future Direction}

To increase power levels, a modular approach using the $2-\mathrm{kW}$ converter as a building block for higher power units will be explored.

\section{Publications}

"A Low Cost, Triple-Voltage Bus DC/DC Converter for Automotive Applications," pp. 1015-21 in IEEE Applied Power Electronics Conference and Exposition (APEC), vol. 2, March 6-10, 2005, Austin, Texas.

"A Small Signal Analysis of A Dual Half Bridge Isolated ZVS Bi-directional dc-dc Converter for Electrical Vehicle Applications," pp. 2777-82 in Proceedings of IEEE PESC'05, June 12-16, 2005, Recife, Brazil.

"A Low Cost, Triple-Voltage Bus DC/DC Converter for Automotive Applications," submitted to IEEE Transactions on Industry Applications. 


\subsection{Integrated Inverter for HEVs and Fuel Cell Powered Vehicles}

Principal Investigator: Gui-Jia Su

Oak Ridge National Laboratory

National Transportation Research Center

2360 Cherahala Boulevard

Knoxville, TN 37932

Voice:865-946-1330; Fax:865-946-1400;E-mail:sugj@ornl.gov

DOE Technology Development Manager: Susan A. Rogers

Voice:202-586-8997;Fax: 202-586-1600; E-mail:Susan.Rogers@ee.doe.gov

ORNL Program Manager: Mitch Olszewski

Voice:865-946-1350; Fax:865-946-1262; E-mail: olszewskim@ornl.gov

\section{Objectives}

- Develop integrated power conversion topologies to reduce the component count, size, and cost of power electronics systems for traction drives and compressor motor drives in hybrid electric vehicles (HEVs) and fuel cell-powered vehicles (FCVs) by reducing the cost and size of the compressor drive by $30 \%$.

- Produce a 75-kW/5-kW dual inverter prototype and demonstrate its capability to independently control three-phase main and two-phase auxiliary permanent magnet (PM) motors.

\section{Approach}

- In order to reduce the cost of a compressor drive in HEVs and FCVs, a two-phase motor and inverter was employed to reduce the manufacturing cost of the motor as a result of eliminating one stator phase winding. While a stand-alone two-phase inverter may require three or four phase legs, the number of phase legs was reduced to two by integrating the inverter into the three-phase inverter of the traction motor drive, yielding a 1/3 reduction in the number of switches and associated gate driver components compared with a three-phase inverter. The integration further enables sharing of the dc bus filter capacitors, gate drive power supplies, and control circuitry between the two inverters. In short, a 30\% reduction in compressor drive cost and size can be achieved through the use of this concept.

\section{Major Accomplishments}

- To demonstrate the size reduction of the compressor drive and to experimentally verify the capability of independently controlling two motors, a $75-\mathrm{kW} / 5-\mathrm{kW}$ dual inverter prototype was designed and built using the latest trench-gate insulated gate bipolar transistor (IGBT) power modules. A new digital signal processing (DSP) control board based on a Texas Instruments chip has also been designed and fabricated for controlling the IGBTs in the dual inverter. Testing of the inverter controlling two PM motors was completed successfully. A $30 \%$ reduction in the compressor drive's component cost and size was achieved compared with a three-phase alternative approach. 


\section{$\underline{\text { Technical Discussion }}$}

\section{Background}

Traditionally, in vehicles powered by internal combustion engines, the engine drives the compressor for heating, ventilating, and air-conditioning (HVAC). The efficiency of the air-conditioning system is not optimized, as the compressor is operated in repetitive run-and-stop modes according to the thermostat settings, and must follow engine speeds. Switching to a motor-driven compressor allows the use of continuously adjustable speed control to reduce air-conditioner energy consumption and enhance HVAC performance by changing the compressor speed according to the cooling/heating requirements. Because of their superior performance compared with their conventional engine-belt-driven counterparts, electricmotor-driven compressors are being deployed in automobiles with a 42-V power net and in HEVs where a high-voltage bus is readily available. Other advantages of electrically driven HVAC compressors are that

- The operating speed can be increased to a much higher level to substantially reduce the size of the motor and compressor.

- The packaging is more flexible, as the location is not restricted to the accessory drive side of the engine.

- Refrigerant leakage into the atmosphere is reduced because rotating seals are eliminated.

- Tailpipe emissions are reduced and fuel economy is improved because the electric compressor enables HEVs to shut off the engine during vehicle stops or at low vehicle speeds when engine power is not required.

Moreover, FCVs require an electrically-driven HVAC compressor.

Figure 1 shows an existing configuration in HEVs that employ two separate three-phase inverter drives for traction and compressor motors. To reduce the cost of the automotive accessory drives such as compressor motors, two-phase inverter-fed induction motor drives have been used to replace wound-field or PM dc motors for heating, ventilating, demisting, engine cooling, and water-pumping applications in the automotive industry. A two-phase motor can be controlled by either a four-leg inverter or a lower-cost two-leg inverter plus a split-capacitor leg, as illustrated in Figure 2. A three-phase motor fed by a threephase inverter typically requires a three-leg inverter employing six switches. Unlike a semiconductor switch leg, the split-capacitor leg does not need additional gate drive or control circuits. Capacitors, however, have their own drawbacks, such as lower reliability and a short service lifespan. These drawbacks become aggravated by the harsh environments expected in HEV/FCV applications. It is therefore desirable to eliminate the split-capacitor leg. 


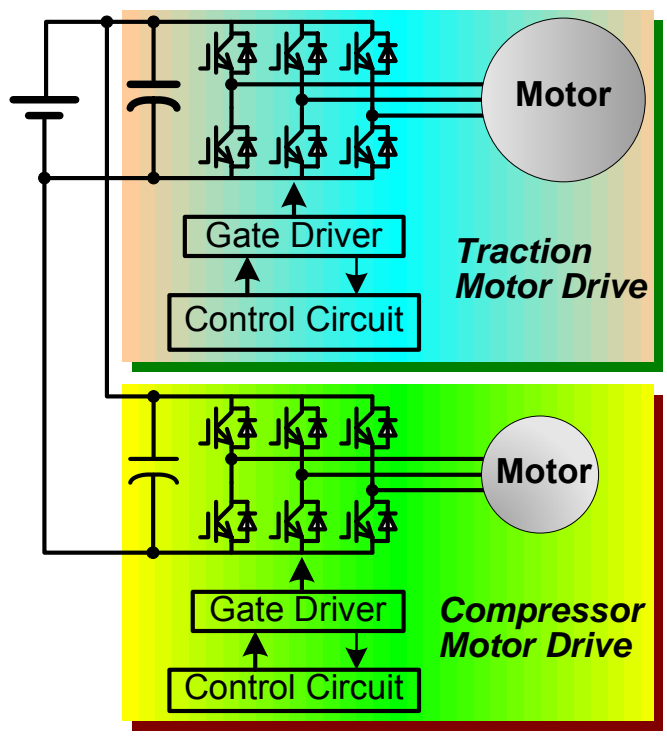

Figure 1. Existing configuration employing two separate three-phase inverter drives for traction and compressor motors in HEVs.

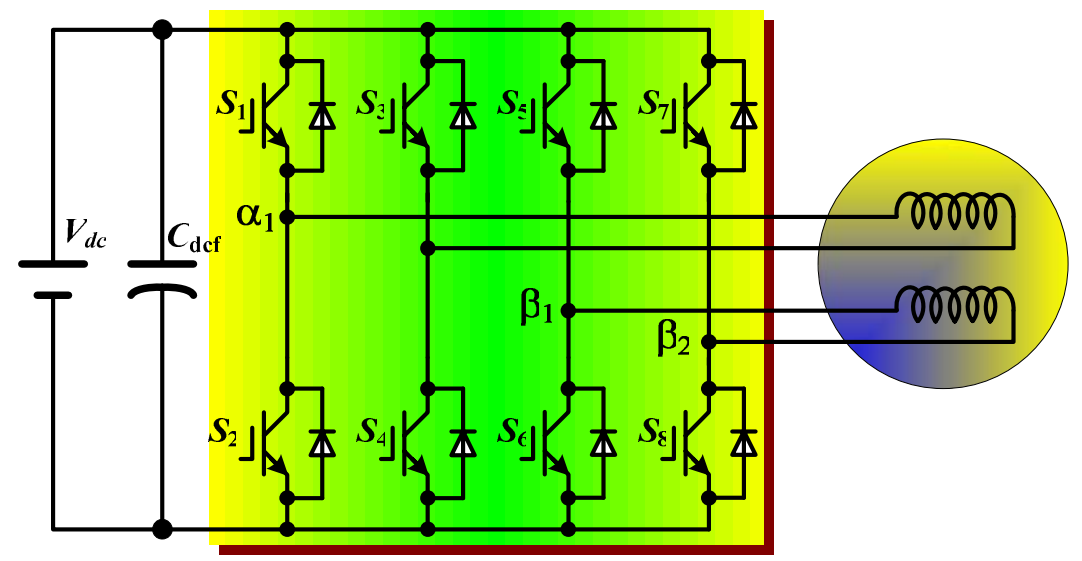

(a) Four-leg two-phase inverter

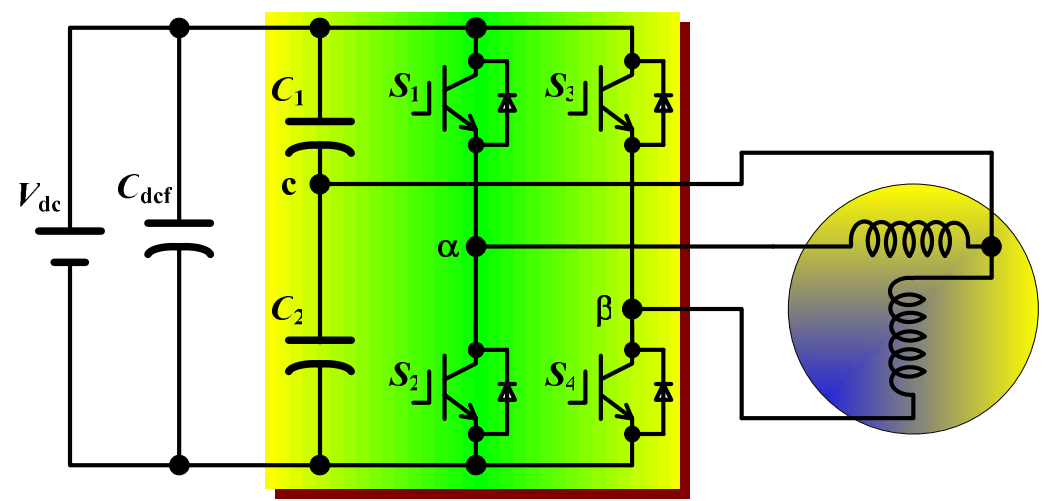

(b) Three-leg two-phase inverter

Figure 2. Possible two-phase motor drive configurations. 
In this project, an integrated dual inverter with five legs has been developed for speed control of a three-phase traction motor and a two-phase compressor motor to further reduce the compressor drive cost. The two-phase inverter is first integrated into the three-phase inverter for the traction motor, so the dc bus filter capacitors, gate drive power supplies, and control circuit can be shared. Furthermore, the splitcapacitor leg is eliminated by tying the common terminal of the two-phase motor to the neutral point of the three-phase traction motor. Integrating the compressor drive into the traction motor drive in this fashion results in a lower-cost, smaller-volume drive system. Both simulation studies and prototype testing with induction motors conducted in the previous year have verified the independent speed control of the two motors. While induction motors cost less, PM motors provide higher efficiency and power density. They also have different control requirements. This report concentrates on the design, fabrication, and testing of a new $75-\mathrm{kW} / 5-\mathrm{kW}$ dual inverter controlling two PM motors.

\section{Integrated Dual Inverter Topology}

\section{Description of the intergrated dual inverter}

Figure 3 shows the proposed five-leg integrated inverter for driving a three-phase traction motor and a two-phase compressor motor. The inverter consists of a dc source, $\mathrm{V}_{\mathrm{dc}}$; a filter capacitor, $\mathrm{C}_{\mathrm{dcf}}$; and five phase legs, $\mathrm{U}, \mathrm{V}$, and $\mathrm{W}$ for feeding the traction motor, and a and $\mathrm{b}$ for the compressor motor. The twophase motor has two windings, phase-a and phase-b; and the two phase windings are connected at one end to form a common terminal, $\mathrm{T}_{\text {com }}$, with the other ends remaining separated to form two independent phase terminals, $\mathrm{T}_{\mathrm{a}}$ and $\mathrm{T}_{\mathrm{b}}$.

The first three legs of the inverter- $\mathrm{U}, \mathrm{V}$, and $\mathrm{W}$, consisting of the switches $\mathrm{S} 1 \sim \mathrm{S} 6$ - form the threephase main inverter, which through pulse-width modulation (PWM) provides three sinusoidal currents to the three-phase motor. The remaining two legs, a and b, are connected to the independent phase terminals of the two-phase motor, $\mathrm{T}_{\mathrm{a}}$ and $\mathrm{T}_{\mathrm{b}}$, respectively, forming an auxiliary two-phase inverter. In addition, the common terminal, $\mathrm{T}_{\text {com }}$, is connected to the neutral point, $\mathrm{N}$, of the three-phase motor to eliminate the split-capacitor phase leg. The two phase legs, a and b, use PWM to provide two sinusoidal currents with a phase shift of 90 electrical degrees from the two-phase motor. The sum of the two-phase currents, $i_{a}$ and $\mathrm{i}_{\mathrm{b}}$, will split evenly into three parts; each part flows through one of the phase windings of the three-phase motor and the associated phase leg of the three-phase inverter as the return path.

Figure 4(a) shows an equivalent circuit of the integrated drive system, in which the inverter is represented by five voltage sources $-\mathrm{v}_{\mathrm{u}}, \mathrm{v}_{\mathrm{v}}, \mathrm{v}_{\mathrm{w}}, \mathrm{v}_{\mathrm{a}}$ and $\mathrm{v}_{\mathrm{b}}$ - corresponding to the five phase legs, $\mathrm{U}, \mathrm{V}, \mathrm{W}$, $a$, and $b$, respectively. All the voltage sources are referred to the midpoint of the dc source, $V_{d c}$. By connecting the common terminal, $\mathrm{T}_{\text {com }}$ to the neutral point, $\mathrm{N}$, of the three-phase motor, the sum of the two-phase currents, $i_{N}\left(=i_{a}+i_{b}\right)$, will split evenly into three parts. Each part will flow through one of the phase windings of the three-phase motor and the associated phase leg of the three-phase inverter as the return path, assuming a symmetrical three-phase motor and inverter. The two-phase motor currents are therefore zero-sequence components flowing in the three-phase stator and will have no effect on the operation of the three-phase motor because the zero-sequence currents will not produce torque, as shown in Figure 4(b). In other words, the torque-producing currents of the two motors can be controlled independently.

In Figure 4(b), the zero-sequence circuit (ZSC) of the three-phase stator is separated from the positive and negative sequence circuits, where $\mathrm{R}_{\mathrm{ms}}$ and $\mathrm{l}_{\mathrm{m} 0 \mathrm{~s}}$ represent the resistance and inductance of the ZSC, and $\mathrm{v}_{0}$ is the zero-sequence component of the three-phase voltage sources, $\mathrm{v}_{\mathrm{u}}, \mathrm{v}_{\mathrm{v}}$, and $\mathrm{v}_{\mathrm{w}}$, which may or may not exist depending on the PWM scheme. The zero-sequence voltage, $\mathrm{v}_{0}$, can be calculated by

$$
v_{0}=\frac{v_{u}+v_{v}+v_{w}}{3}
$$




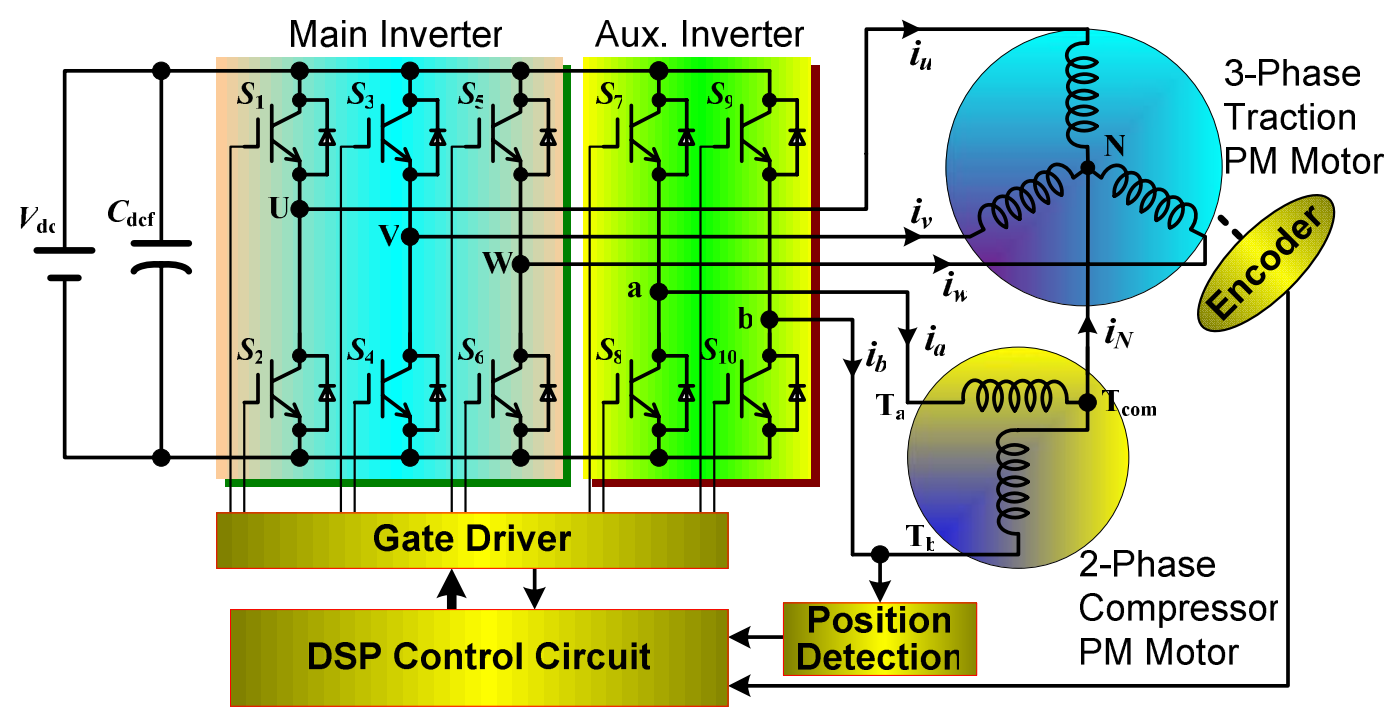

Figure 3. Proposed integrated inverter for driving a three-phase traction motor and a two-phase compressor motor.

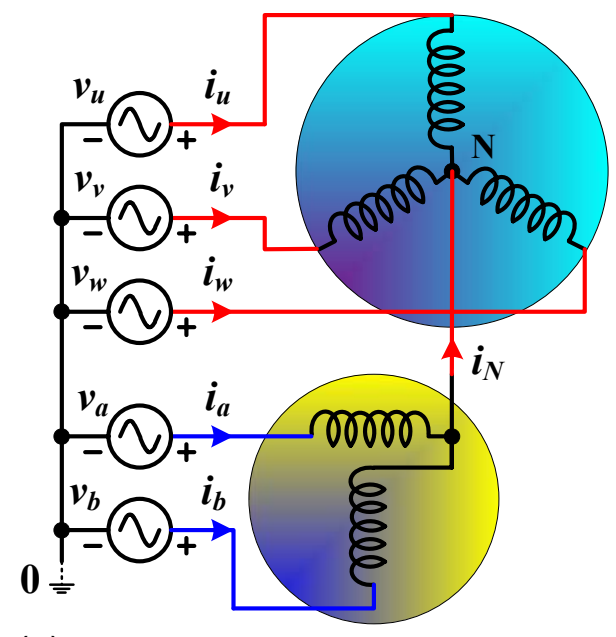

(a)

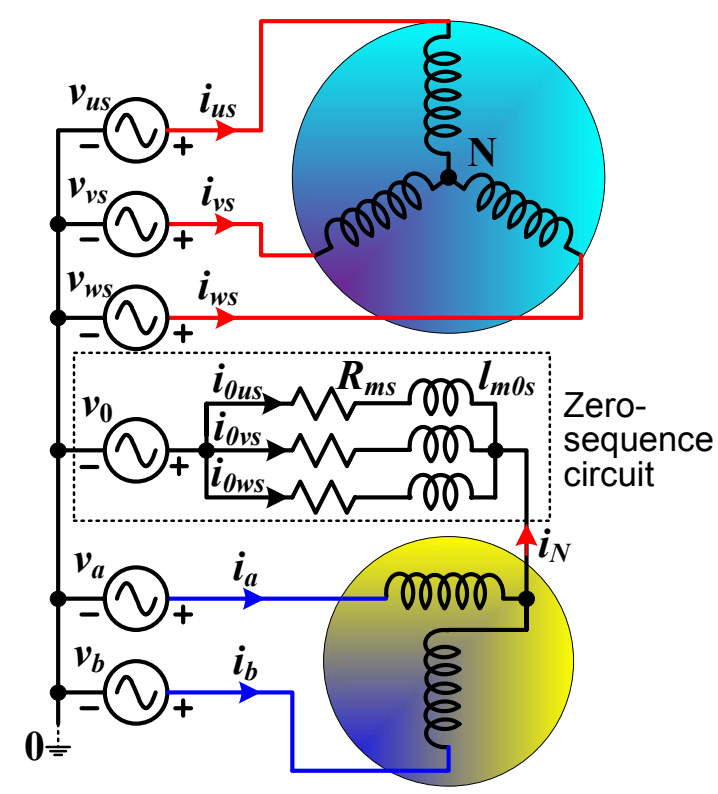

(b)

Figure 4. Equivalent circuits showing (a) inverter phase legs as voltage sources and (b) the zero-sequence circuit of the main motor as the current return path of the two-phase motor. 
The terms $\mathrm{v}_{\mathrm{us}}, \mathrm{v}_{\mathrm{vs}}$, and $\mathrm{v}_{\mathrm{ws}}$ are the phase voltages referenced to the zero-sequence voltage of the three phases $\mathrm{U}, \mathrm{V}$, and $\mathrm{W}$, respectively, and are expressed by

$$
\left\{\begin{array}{l}
v_{u s}=v_{u}-v_{0} \\
v_{v s}=v_{v}-v_{0} \\
v_{w s}=v_{w}-v_{0} .
\end{array}\right.
$$

The zero-sequence voltage component, $\mathrm{v}_{0}$, which could be generated by certain PWM strategies such as space vector modulation schemes, can be cancelled by injecting the same component into the modulation signals for the two-phase inverter so that $\mathrm{v}_{0}$ will not produce current in the circuit, as will be shown in the simulation and experimental results.

\section{Effects on the current rating of the main motor}

Because the stator windings of the three-phase motor are used as the current return paths of the twophase motor, the stator current rating may need be increased to accommodate the two-phase motor currents. However, the increase of the main motor current is negligible if the two-phase motor current is sufficiently small compared with that of the main motor, which is typical in the intended automotive applications, as shown below.

The phase- $U$ current of the main motor $i_{u}$ can be expressed by

$$
i_{u}=i_{u s}-\frac{i_{a}+i_{b}}{3}
$$

where $i_{\text {us }}$ is the required current if the three-phase motor is operated alone without connection to the two-phase motor. Because the two motor currents will usually have different frequencies, the $r_{\mathrm{ms}}$ value of the main motor phase current, $i_{u}$, can therefore be calculated by

$$
I_{u, r m s}=\sqrt{I_{u s, r m s}^{2}+\frac{2 I_{a, r m s}^{2}}{9}}
$$

where $I_{a, r m s}$ is the required rms current of the two-phase motor. For instance, given a $350-A_{r m s}$ traction motor and a $25-\mathrm{A}_{\mathrm{rms}}$ compressor motor, i.e., $\mathrm{I}_{\mathrm{us}, \mathrm{rms}}=350 \mathrm{~A}$ and $\mathrm{I}_{\mathrm{a}, \mathrm{rms}}=25 \mathrm{~A}$, the resulting traction motor current is

$$
\begin{aligned}
I_{u, r m s} & =\sqrt{350^{2}+\frac{2 \times 25^{2}}{9}} \\
& =350.2 \mathrm{Arms}
\end{aligned}
$$

giving a negligible increase of $0.2 \mathrm{~A}$, less than $0.06 \%$.

\section{Component count reduction}

Compared with the conventional system consisting of two separate three-phase inverters, one phaseleg including two switches and their associated gate drivers can be eliminated. Moreover, it is apparent that by integrating the two-phase auxiliary inverter into the main three-phase inverter, the dc bus filter capacitor and gate drive power supplies can be shared between the two inverters. Furthermore, a single control circuit typically based on a microprocessor or digital signal processor (DSP) with built-in motor control hardware—-such as a/a converters, PWM counters, and encoder interface circuitry—can be used to 
execute control algorithms for the two motors. With a proper control algorithm, the motors can be run in either motoring mode. i.e., providing power to the motor shaft, or generating mode, in which power is transferred from the motor shaft to the inverter dc source. In short, for the compressor drive, the inverter components including semiconductor switches and gate drive circuits can be reduced by more than onethird.

\section{Inverter Design, Test Setup and Experimental Results \\ Inverter design and test setup}

A prototype inverter rated at $75 \mathrm{~kW}$ for the traction drive and $5 \mathrm{~kW}$ for the compressor drive was designed and fabricated. The design uses the latest trench-gate 600-V/600- six-pack IGBT intelligent module from POWEREX and two 600-V/75-A dual-pack IGBTs, which are mounted on a water-cooled heat sink with a footprint of $12 \times 7 \mathrm{in}$., as shown in Figure 5. Four film capacitors rated at $600 \mathrm{~V} / 260 \mu \mathrm{F}$ are employed in the dc bus filter. A new DSP control board based on a TI chip, TMS320F2812, has been designed and fabricated for controlling the IGBTs. The DSP chip has two sets of PWM hardware and thus can control two motors simultaneously. Figure 6 shows a photo of the complete inverter assembly with the DSP control board, a main gate driver board underneath the DSP board, an auxiliary gate driver board for the dual-pack IGBTs, and a control power supply. The assembly has a footprint of $12 \mathrm{in}$. width $\times$ 11 in. depth and a volume of 385 in. $_{.3}$, of which over one-third, 149 in. $_{.3}$, is occupied by the capacitors.

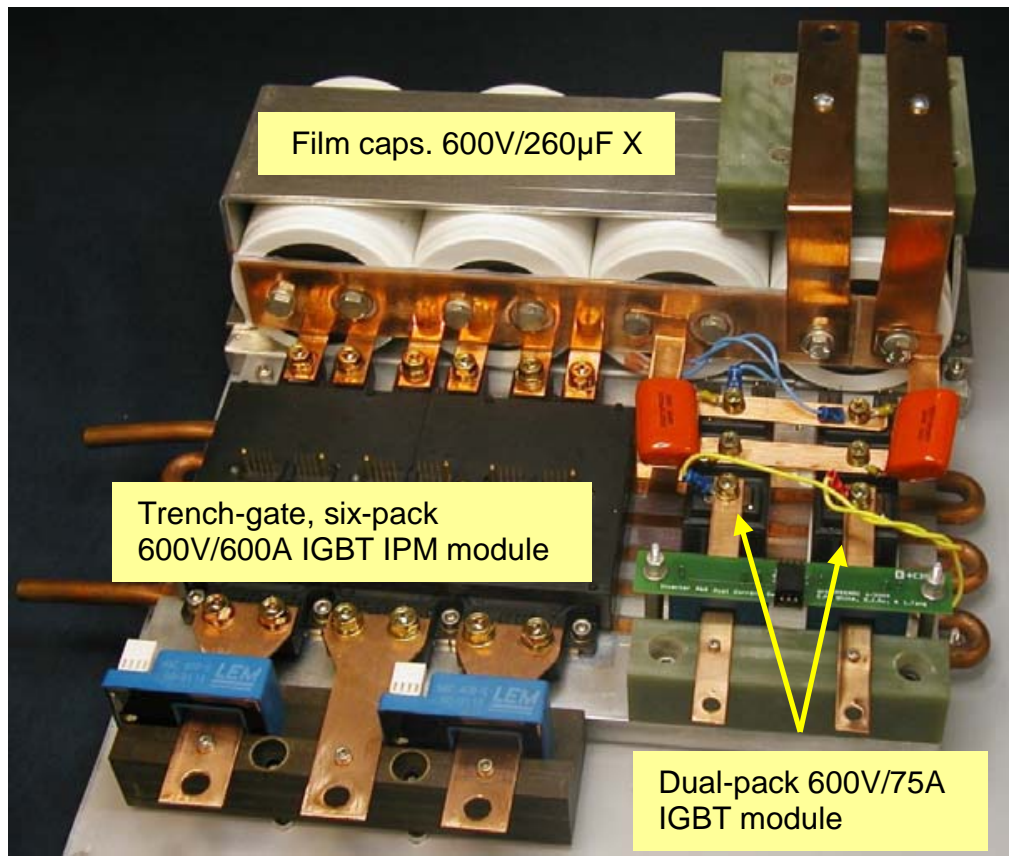

Figure 5. Dual inverter prototype without the gate driver and control boards. 


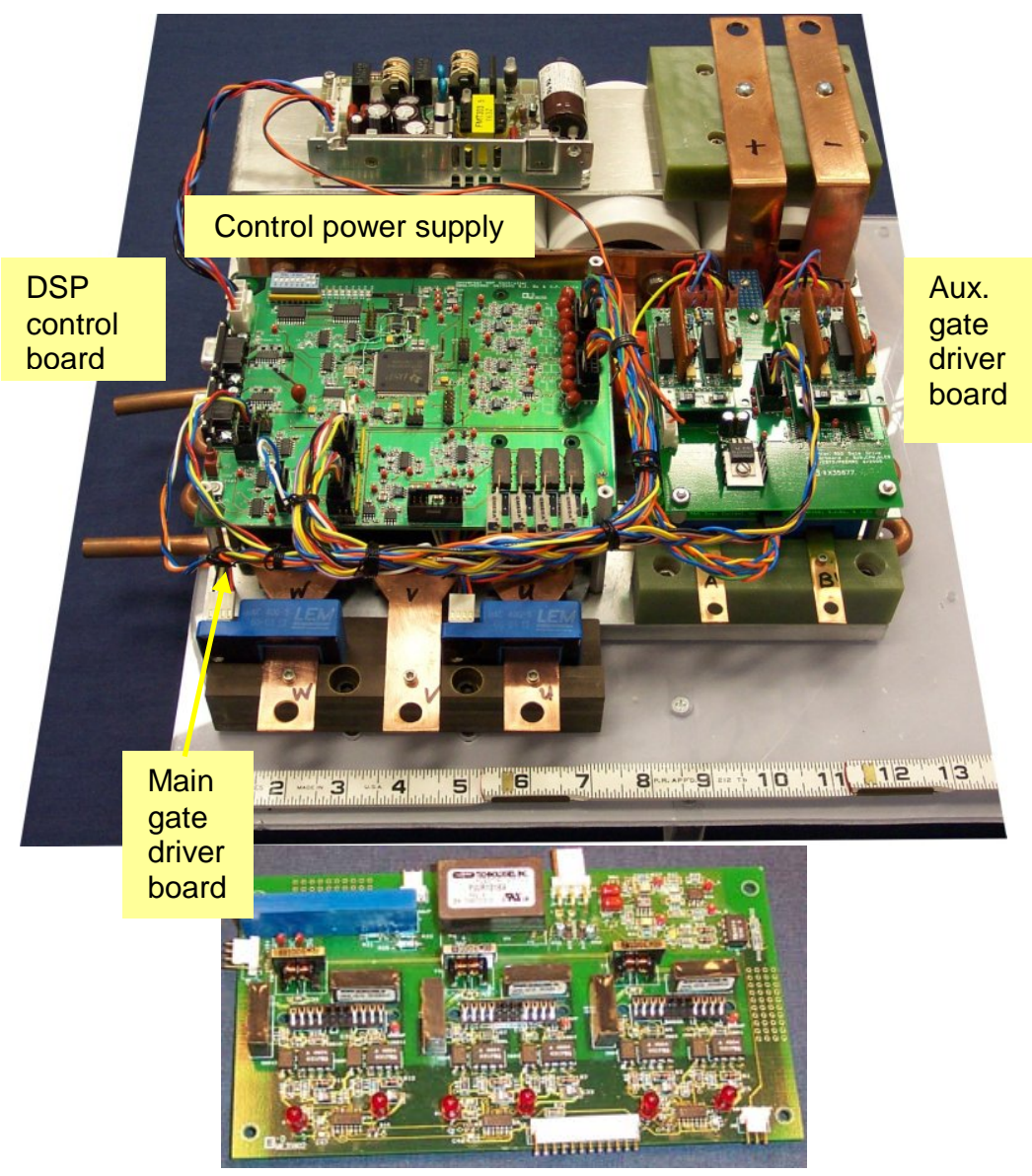

Figure 6. Dual inverter prototype-complete assembly.

Figures 7-9 show the test setup of the inverter and motors. For testing, a three-phase PM motor with eight poles, rated at $8.2 \mathrm{~kW}$ and $2000 \mathrm{rpm}$, was used as the traction motor because a PM motor with a power and speed range that meets FreedomCAR motor requirements was not readily available when the design and testing were conducted. For the compressor motor, a three-phase, eight-pole PM motor rated at $5.5 \mathrm{~kW}$ and $4000 \mathrm{rpm}$ was modified to form a two-phase motor, as shown in Figure 10(a), in which phase-b and phase-c windings are connected in series. This connection results in an equivalent asymmetrical two-phase PM motor with two orthogonal windings with a turns ratio of $\sqrt{3}: 1$, as can be seen from the magnetomotive force vectors shown in Figure 10(b). 


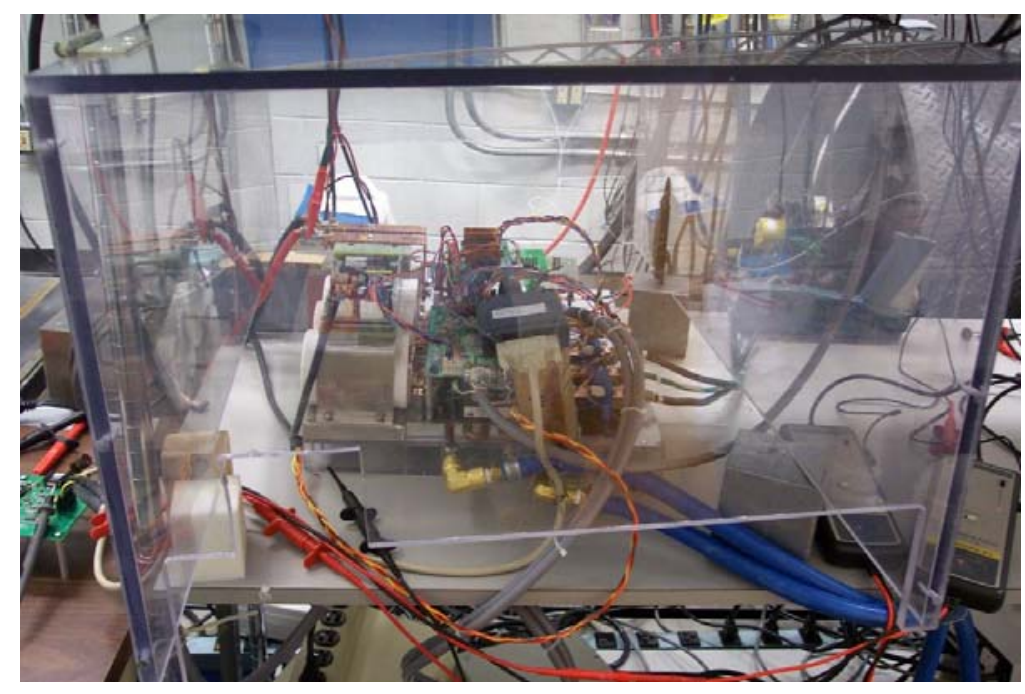

Figure 7. Dual inverter prototype connected for testing.

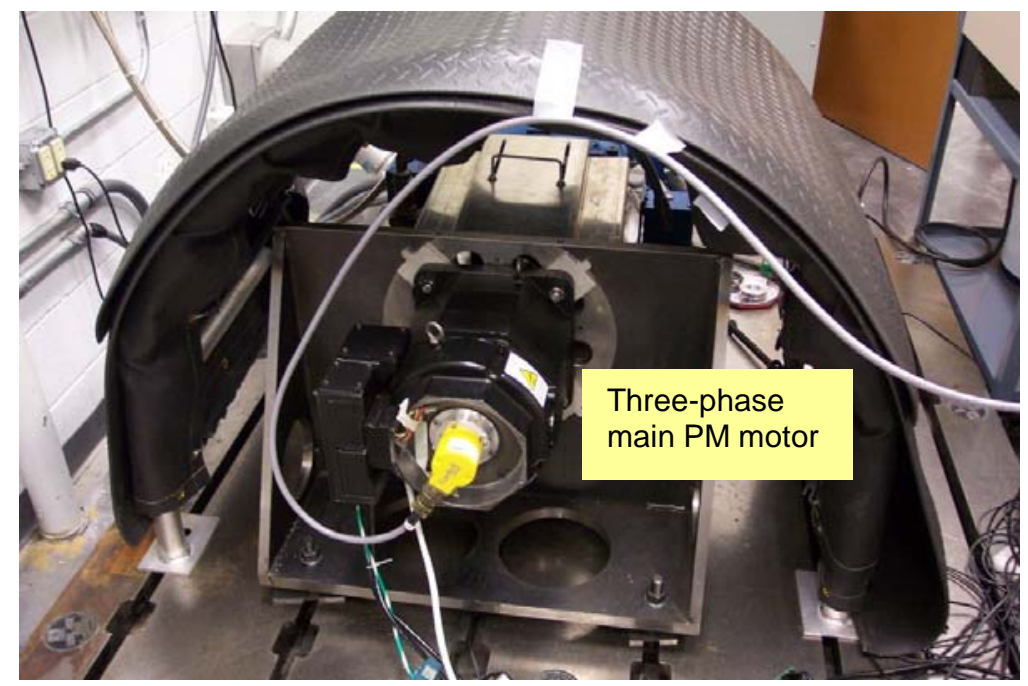

Figure 8. Three-phase main PM motor mounted on the motor test bed with a 100-HP dyne. 


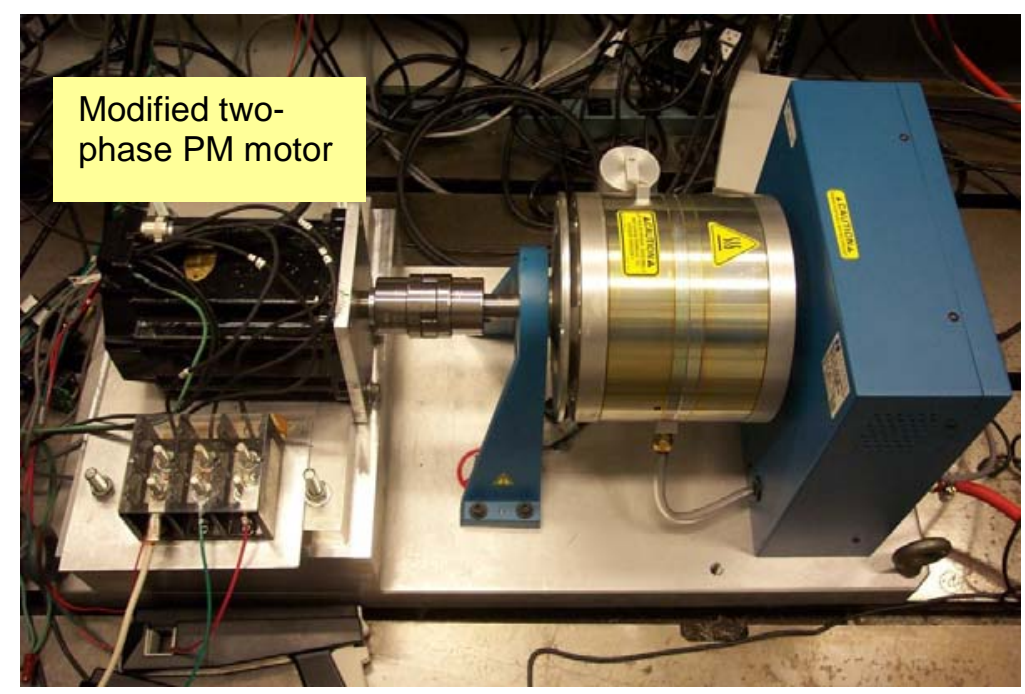

Figure 9. Two-phase PM motor mounted on a portable 5-HP eddy current dyne motor test bed.

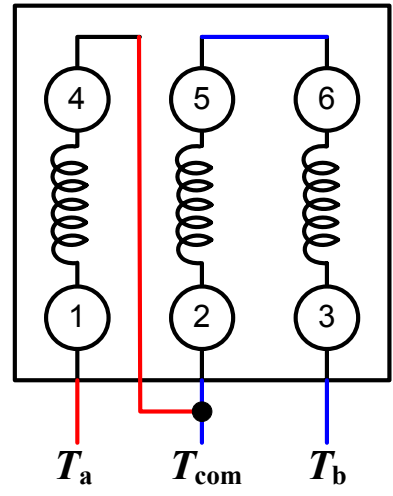

(a)

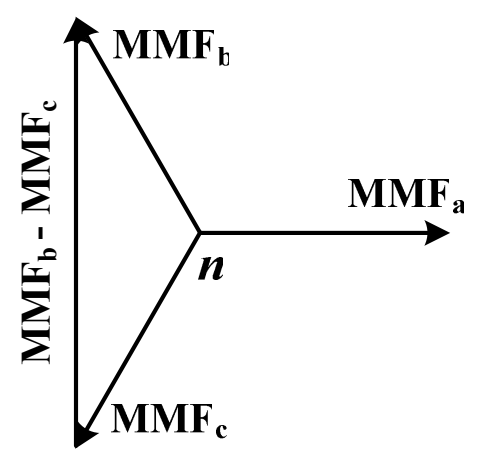

(b)

Figure 10. Modification of the three-phase motor into a two-phase one.

\section{Experimental results}

Extensive testing was conducted to verify the integrated drive operations. Figures 11-16 give testing waveforms at various load conditions, which clearly show that the speed of the two motors can be controlled independently. In Figures 11 and 12, the main motor was not running while the two-phase motor was loaded with $10 \mathrm{~N} \cdot \mathrm{m}$ at $505 \mathrm{rpm}$ and $7.5 \mathrm{~N} \cdot \mathrm{m}$ at $1000 \mathrm{rpm}$, respectively. In Figures 13 and 14, the two-phase motor was stopped while the main motor was loaded with the rated torque of $40 \mathrm{~N} \cdot \mathrm{m}$ at $500 \mathrm{rpm}$. In Figure 13, the speed was increased to $1852 \mathrm{rpm}$ and the load torque to $43 \mathrm{~N} \cdot \mathrm{m}$. Figure 14 shows the unit delivering the rated power of $8.2 \mathrm{~kW}$. In Figure 15, the main motor was loaded with 40 $\mathrm{N} \cdot \mathrm{m}$ at $750 \mathrm{rpm}$, while the two-phase motor was loaded with $12 \mathrm{~N} \cdot \mathrm{m}$ at $505 \mathrm{rpm}$. In Figure 16, the main motor was loaded with $40 \mathrm{~N} \cdot \mathrm{m}$ at $1000 \mathrm{rpm}$, while the two-phase motor was loaded with $12 \mathrm{~N} \cdot \mathrm{m}$ at 800 $\mathrm{rpm}$. It should be noted that because of their asymmetrical windings, the two-phase motor currents, $\mathrm{i}_{\mathrm{a}}$ and $\mathrm{i}_{\mathrm{b}}$, are not equal. 


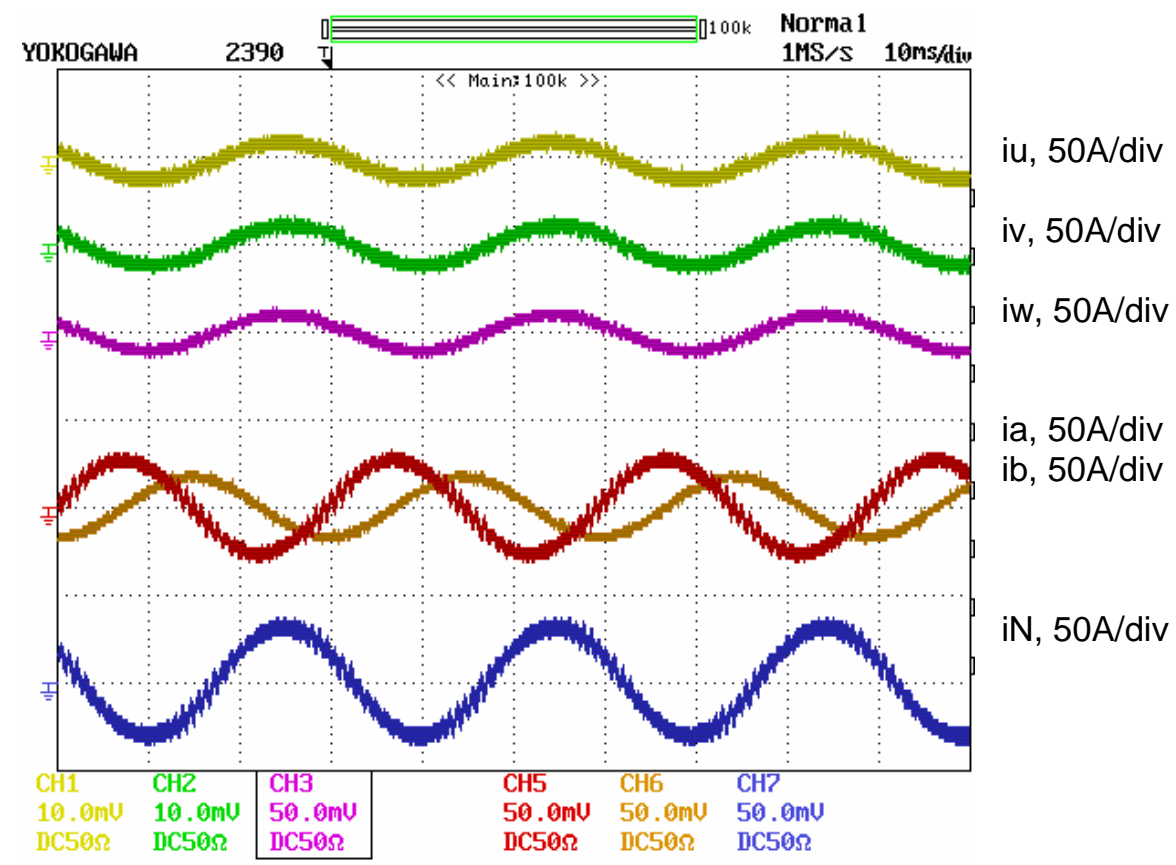

Figure 11. The main motor is not running while two-phase motor loaded with $10 \mathrm{~N} \cdot \mathrm{m}$ at $505 \mathrm{rpm} .10 \mathrm{~ms} / \mathrm{div}$.

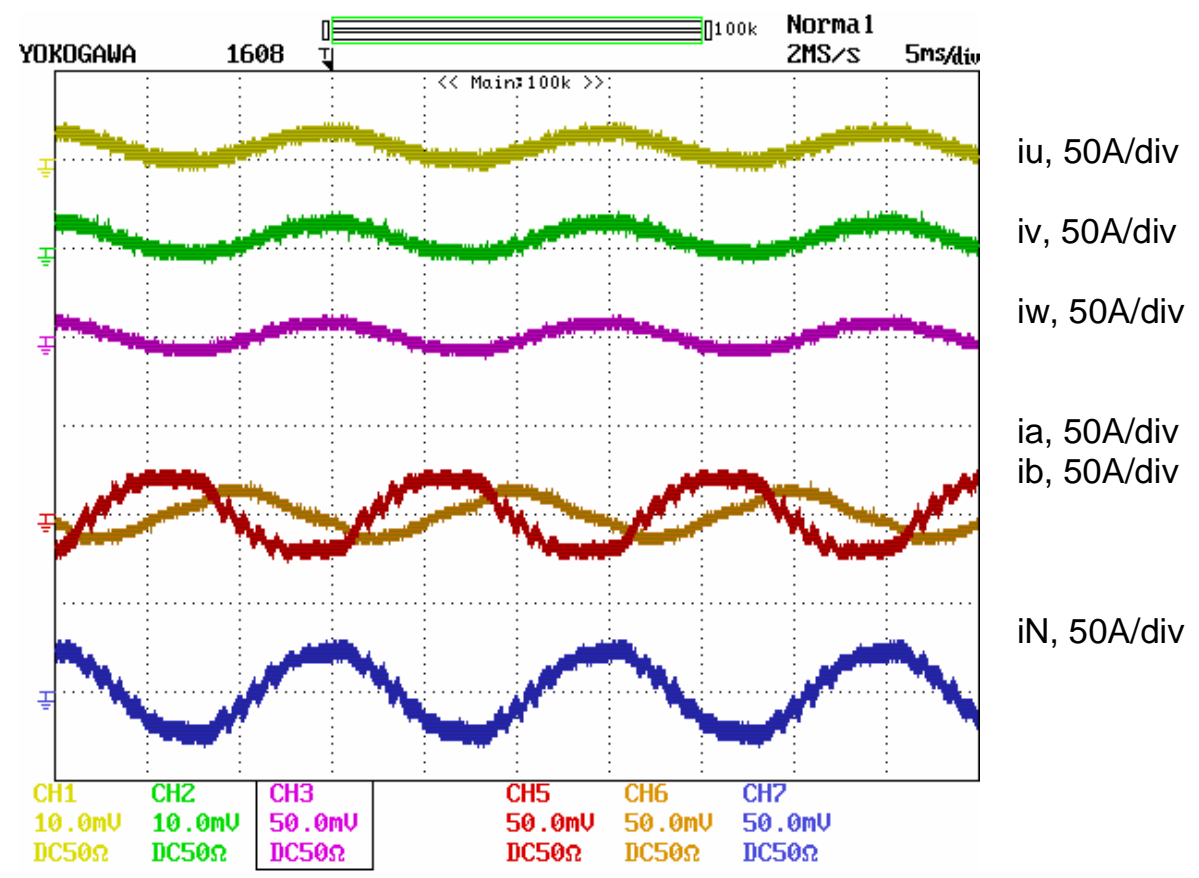

Figure 12. The main motor is not running while two-phase motor loaded with $7.5 \mathrm{~N} \cdot \mathrm{m}$ at $1000 \mathrm{rpm} .5 \mathrm{~ms} / \mathrm{div}$. 


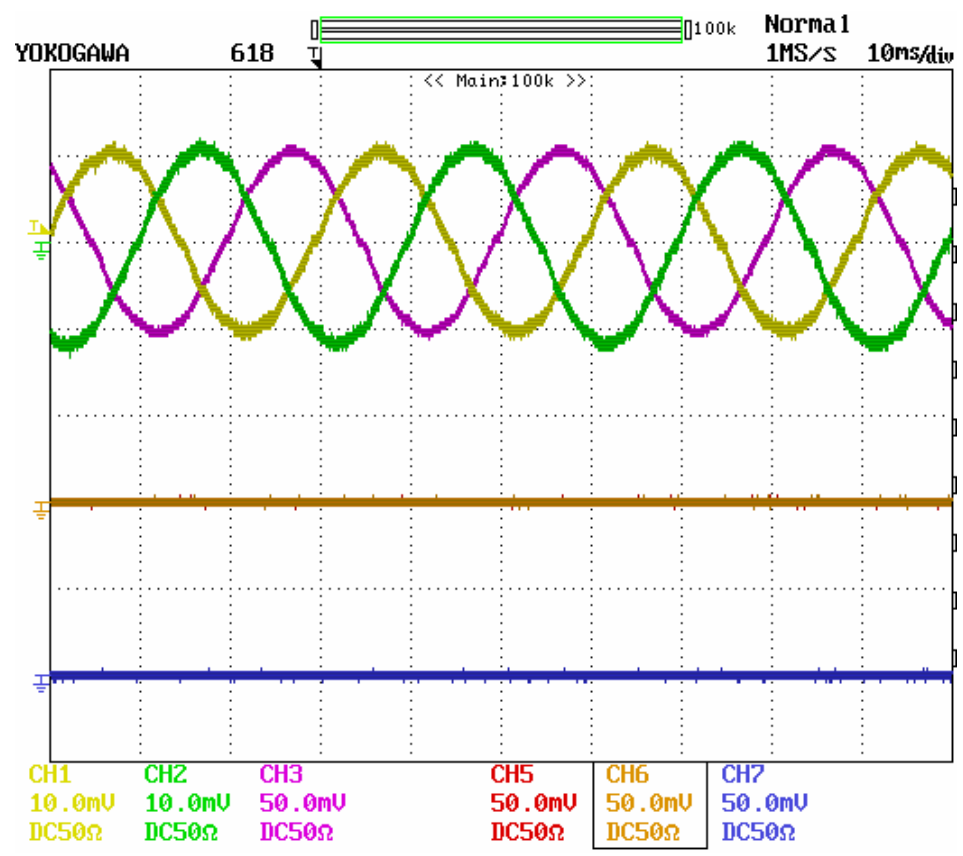

iu, 50A/div

iv, 50A/div

iw, 50A/div

ia, 50A/div

$\mathrm{ib}, 50 \mathrm{~A} / \mathrm{div}$

iN, 50A/div

Figure 13. The two-phase motor is not running while the main motor is loaded with $40 \mathrm{~N} \cdot \mathrm{m}$ at $500 \mathrm{rpm}$. 10ms/div.

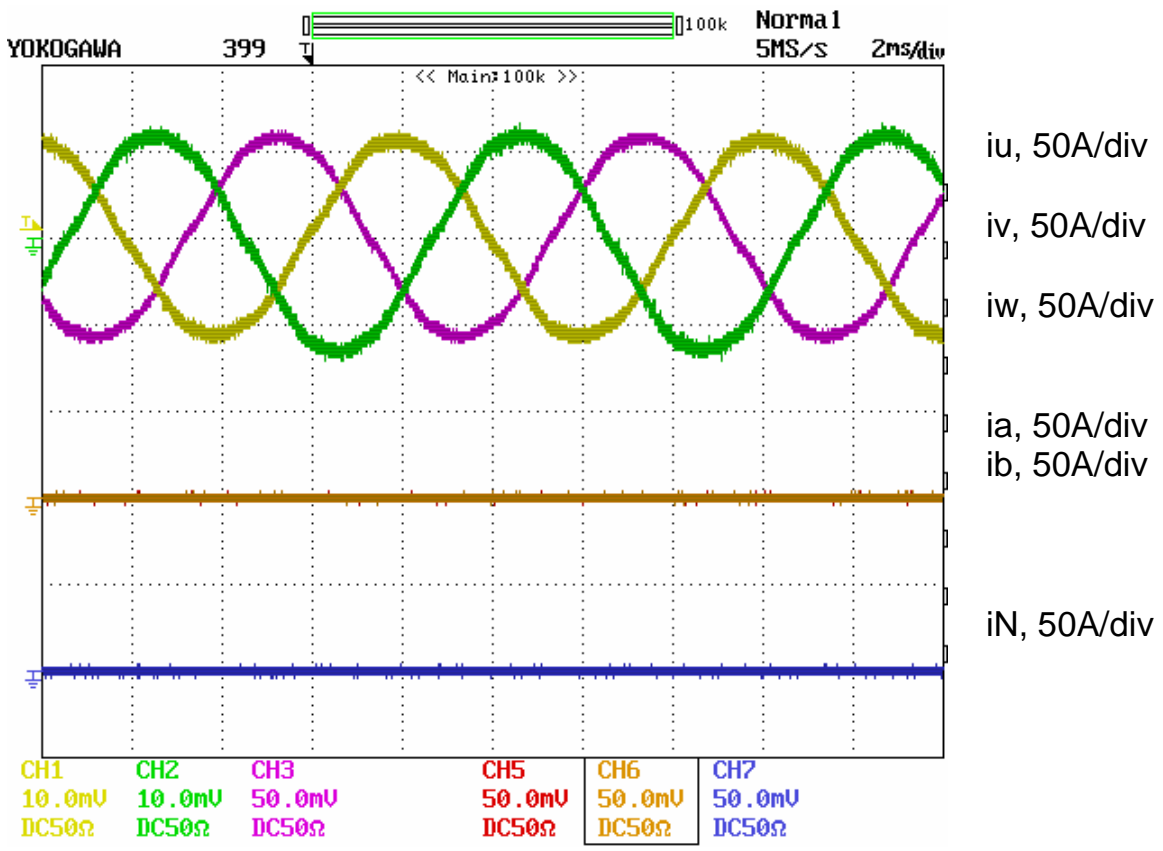

Figure 14. The two-phase motor is not running while the main motor loaded with $43 \mathrm{~N} \cdot \mathrm{m}$ at $1852 \mathrm{rpm}$. $2 \mathrm{~ms} / \mathrm{div}$. 


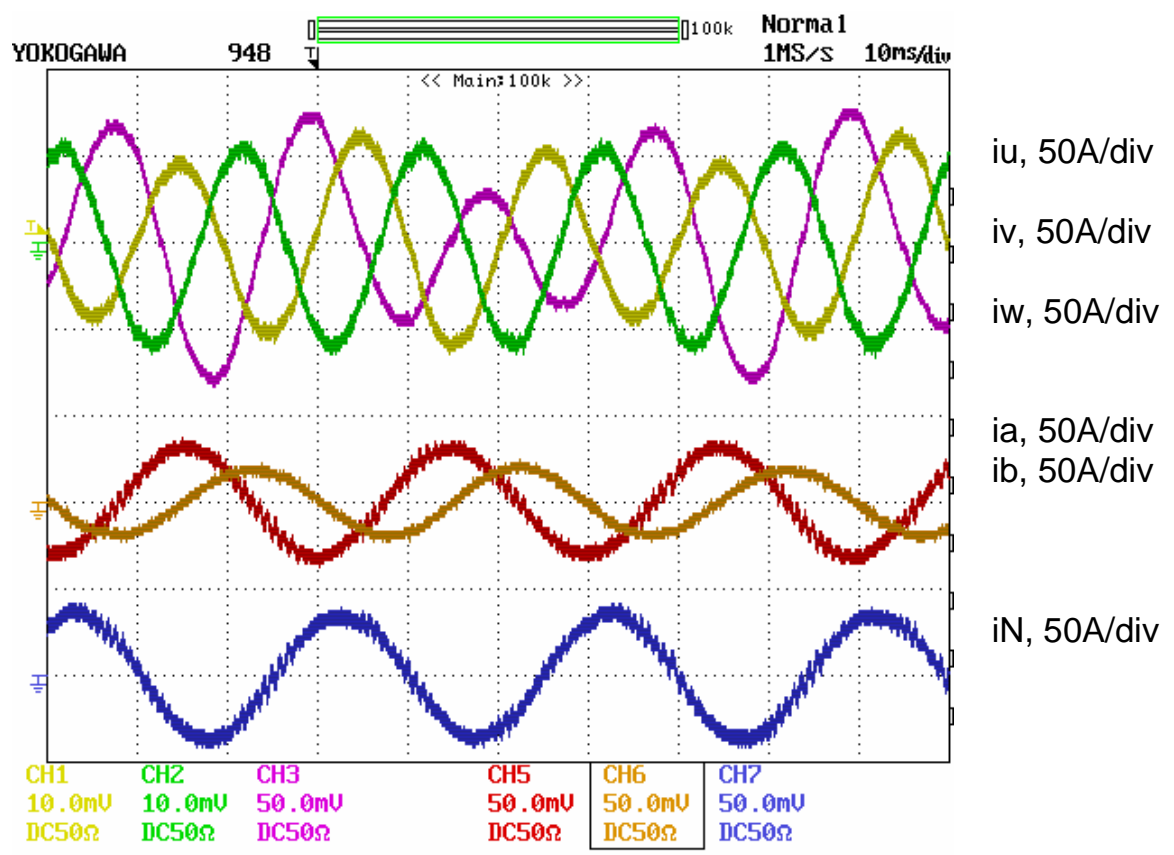

Figure 15. The main motor is loaded with $40 \mathrm{~N} \cdot \mathrm{m}$ at $750 \mathrm{rpm}$, and the two-phase motor is loaded with $12 \mathrm{~N} \cdot \mathrm{m}$ at $505 \mathrm{rpm} .10 \mathrm{~ms} / \mathrm{div}$.

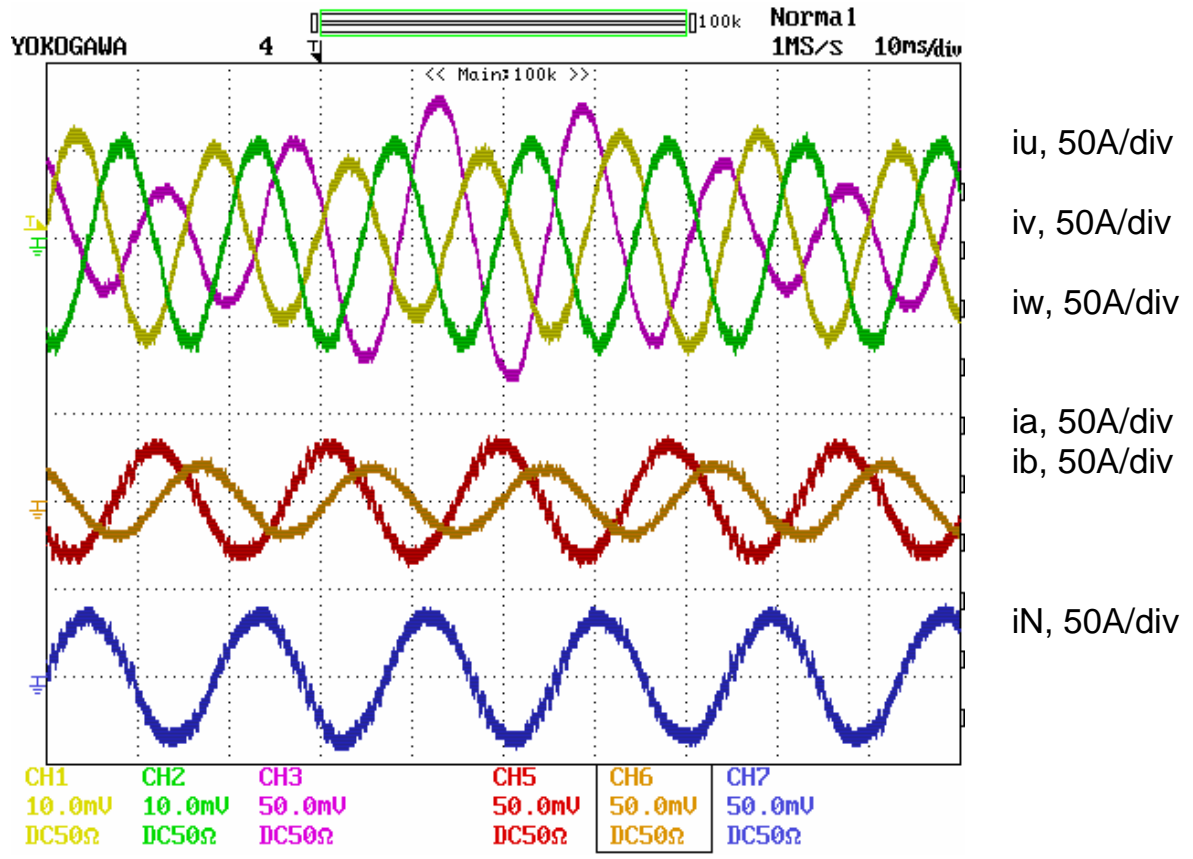

Figure 16. The main motor is loaded with $40 \mathrm{~N} \cdot \mathrm{m}$ at $1000 \mathrm{rpm}$, and the two-phase motor is loaded with $12 \mathrm{~N} \cdot \mathrm{m}$ at $800 \mathrm{rpm} .10 \mathrm{~ms} / \mathrm{div}$. 


\section{Conclusions}

The proposed integrated traction and compressor motor drive employing a five-leg inverter can significantly reduce the cost of the compressor motor drive in HEV/FCV applications. For the compressor drive, the inverter components, including semiconductor switches and gate drive circuits, can be reduced by more than one-third. An additional cost savings is due to the fact that the compressor drive does not require a separate control circuit; it is incorporated into the traction motor controller through software. Finally, the two-phase motor is ultimately cheaper to manufacture because one stator phase winding is eliminated.

The testing results show that

- The split-dc bus capacitors for a two-phase compressor motor drive can be eliminated by using the traction motor stator windings as the current return paths.

- Increase in the current rating of the main inverter switches and the traction motor due to the twophase motor current is negligible.

- The speed of the traction and compressor motors can be controlled independently. The test results on the independent control characteristics of the two motors and on the voltage waveforms agree fully with the analytical predictions.

- The fundamental components of the two motors have no influence on each other.

- The proposed dual inverter is applicable to induction motors, ac synchronous PM machines, and brushless dc motors.

\section{Future Direction}

Secure a PM motor that has power and speed ratings comparable to the FreedomCAR requirements and test the inverter up to its full power capability.

\section{$\underline{\text { Publications }}$}

"A Five-Leg Inverter for Driving a Traction Motor and a Compressor Motor," pp. 117-123 in Proceedings of the 8th IEEE Workshop on Power Electronics in Transportation (WPET2004), Novi, Michigan, Oct. 21-22, 2004.

"An Integrated Traction and Compressor Drive System for EV/HEV Applications," pp. 719-725 in Proceedings of the IEEE Applied Power Electronics Conference and Exposition (APEC), vol. 2, Austin, Texas, March 6-10, 2005.

\section{$\underline{\text { Patents }}$}

"Integrated Inverter for Driving Multiple Motors/Generators," Oak Ridge National Laboratory Invention Disclosure ID 1316, S-101905. 


\title{
4.4 Z-Source Power Converter for Fuel Cell-Powered Vehicles
}

\author{
Donald J. Adams \\ Oak Ridge National Laboratory \\ National Transportation Research Center \\ 2360 Cherahala Boulevard \\ Knoxville, Tennessee 37932 \\ Voice: (865) 946-1321; Fax: (865) 946-1262; adamsdj@ornl.gov \\ DOE Technology Development Manager: Susan A. Rogers \\ Voice: 202-586-8997; Fax: 202-586-1600; E-mail: Susan.Rogers@ee.doe.gov \\ ORNL Program Manager: Mitch Olszewski \\ Voice: 865-946-1350; Fax: 865-946-1262; E-mail: olszewskim@ornl.gov
}

\section{Objective}

- To develop and demonstrate a low-cost, high-efficiency, and reliable inverter for traction drives of fuel cell-powered vehicles.

\section{Approach}

- Investigate the current inverter systems for traction drives and propose a new Z-source inverter traction drive system.

- Perform a comprehensive comparison, model development, and computer simulation of the existing topologies versus the new Z-source inverter.

- Design and prototype a 55-kW Z-source inverter for IM traction drive to prove the concept.

- Perform testing and performance study of the Z-source inverter prototype.

\section{Major Accomplishments}

- In FY 2004, detailed specifications were developed for a Z-source inverter prototype for fuel cell vehicle traction-drive applications. A comprehensive cost comparison, model development, and computer simulation were performed, and a report was issued.

- During FY 2005, a 55-kW prototype was developed and built. Subsequent tests and evaluations at both Michigan State University (MSU) and Oak Ridge National Laboratory (ORNL) proved the viability of the technology to achieve improved efficiencies at reduced cost. Several papers were issued and are listed in the publications section of this report.

\section{Technical Discussion}

Today's power conversion technology is based on the two traditional converter topologies: voltagesource (V-source) and current-source (I-source) converters (or inverters depending on power flow directions). Both the V-source converter and I-source converter have conceptual and theoretical limitations and barriers that prevent economical and efficient solutions to many applications.

A new converter topology and theory called the Z-source power converter was recently invented at MSU. It can overcome some of the problems of the traditional V-source and I-source converters. The Zsource converter is comprised of an impedance network to couple the main converter circuit to the power source or load. The unique feature offered by the Z-source network is that, unlike the traditional V-source or I-source, it can be open and short circuited, which provides a mechanism for the main converter circuit to step up or step down voltage as desired. The Z-source network provides great flexibility for the source, 
main circuit, and load. Because of the wide voltage range of the fuel cell, the traditional inverter and the motor need to be oversized to accommodate the required large constant power speed range. The Z-source inverter could be a cheap and reliable solution for this application.

Great interest was shown by DOE, the national laboratories, and the FreedomCAR Technical Team in further development of the Z-source converter technology. In FY 2004, ORNL entered into an arrangement with MSU to collaborate and fund this development for automotive traction applications.

Currently, two types of inverters are used in fuel cell (FCV) and hybrid electric vehicle (HEV) traction drives: the traditional pulse width modulation (PWM) inverter and the dc/dc-boosted PWM inverter. For FCVs, the fuel cell voltage to the inverter decreases with an increase in power drawn from the fuel cell. Because of the wide voltage change and the limited voltage level of the battery and/or fuel cell stack, the conventional PWM inverter topology imposes high stresses on the switching devices and motor and limits the motor's constant power speed range (CPSR). The dc/dc-boosted PWM inverter topology can alleviate the stresses and limitations; however, it has other problems, such as the high cost and complexity associated with the two-stage power conversion. To demonstrate the superiority of the Zsource inverter for FCVs, a comprehensive analytical comparison of the Z-source inverter versus the two existing inverter topologies, performed using a $50-\mathrm{kW}$ (max) fuel cell stack as the prime energy source and a 34-kW Solectria AC55 induction motor as the traction drive motor, showed that the Z-source inverter can increase conversion efficiency by $1 \%$ over a wide load range, extend CPSR by 1.55 times, and minimize the switching device power rating (SDPR), a cost indicator, by $15 \%$. The three system configurations are shown in Figures 1-3.

In an inverter system, each switching device has to be selected according to the maximum voltage impressed and the peak and average current going through it. To quantify the voltage and current stress (or requirement) of an inverter system, SDPR is used. The SDPR of a switching device/cell is expressed as the product of voltage stress and current stress. The total SDPR of an inverter system is defined as the aggregate of the SDPRs of all the switching devices used in the circuit. Total SDPR is a measure of the total semiconductor device requirement and is thus an important cost indicator for an inverter system.

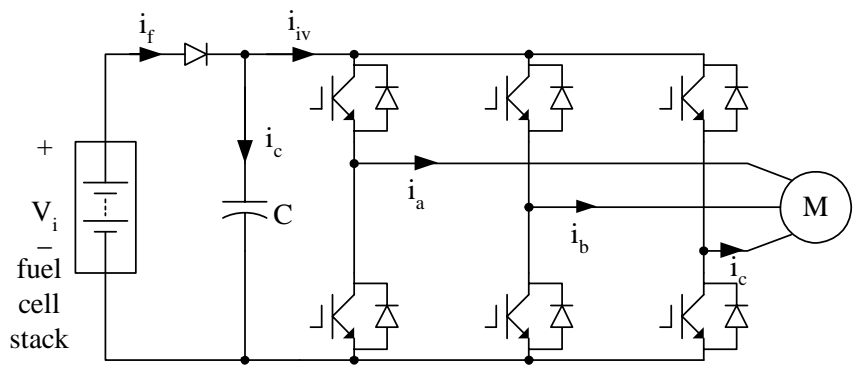

Figure 1. System configuration using conventional PWM inverter.

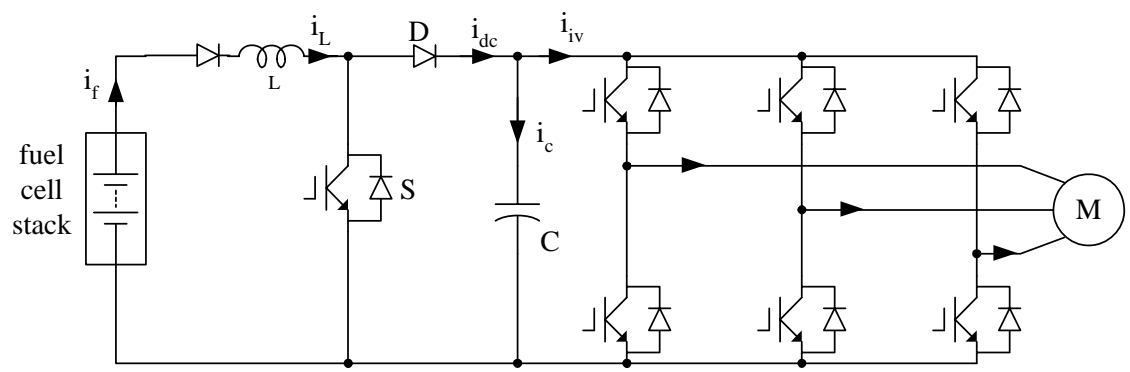

Figure 2. System configuration using dc/dc boost + PWM inverter. 


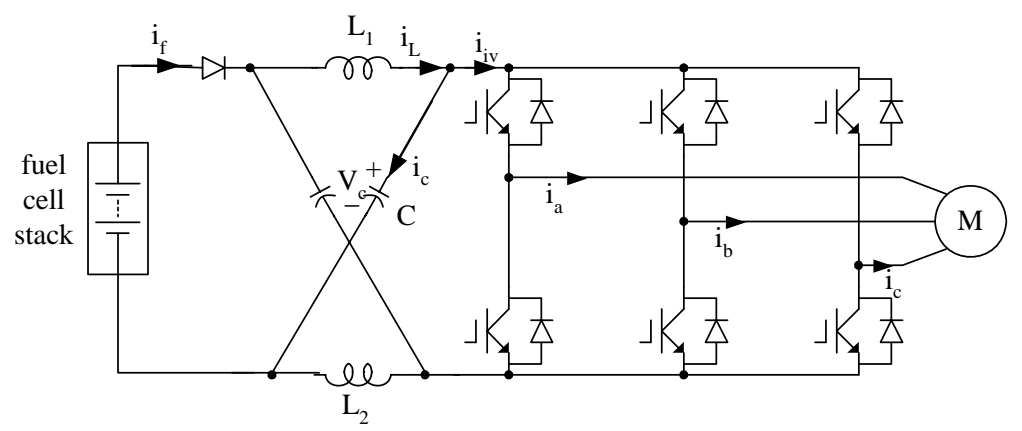

Figure 3. System configuration using the $\mathrm{Z}$ source inverter.

The specifications used in the design, analysis, and prototype development were as follows:

- Continuous power: $30 \mathrm{~kW}$

- Peak power: $55 \mathrm{~kW}$ for 18 seconds

- Inverter efficiency: $>97 \%$ at $30 \mathrm{~kW}$

- Input fuel cell voltage: $0-420 \mathrm{~V} \mathrm{dc}$

$\bullet$

As seen in Table 1, the Z-source inverter's average SDPR is the lowest of the three, and the conventional PWM inverter's SDPRs are the highest in both average and peak values. The average SDPR also indicates thermal requirements and conversion efficiency.

Table 1. Switching device power comparison

\begin{tabular}{|l|c|c|}
\hline Inverter systems & $\begin{array}{c}\text { Total average SDPR } \\
(\mathrm{kVA})\end{array}$ & $\begin{array}{c}\text { Total peak SDPR } \\
(\mathrm{kVA})\end{array}$ \\
\hline PWM inverter & 238 & 747 \\
\hline PWM plus boost dc/dc & 225 & 528 \\
\hline Z-source inverter & 199 & 605 \\
\hline
\end{tabular}

The SDPR numbers are the theoretical ratings of semiconductors required by the three inverter topologies. However, commercially available integrated power modules (IPMs) [or insulated gate bipolar transistors (IGBTs)] are limited in terms of voltage and current ratings. Assuming that the voltage rating is limited to $600 \mathrm{~V}$, and the current rating is chosen to be two times the average current stress of each inverter topology, the selected devices and price quotes from a distributor are listed in Table 2. Again, the Z-source has the lowest price among the three inverters. In addition, because it has fewer components, a higher mean time between failures can be expected, which leads to better reliability. Table 3 shows the comparisons of passives required in the inverter systems to provide a further indication of size and cost.

Efficiency is an important criterion for any power converter. High efficiency can reduce thermal requirements and cost. An efficiency comparison was conducted based on the following conditions: the conventional inverter is always operating at a modulation index of 1 , the $\mathrm{dc} / \mathrm{dc}$ boost plus PWM inverter boosts the dc voltage to $420 \mathrm{~V}$, and the $\mathrm{Z}$-source inverter outputs the maximum obtainable voltage while keeping the switch voltage under $420 \mathrm{~V}$. The same motor model is used to calculate the motor loss.

Table 2. Actual price comparison

\begin{tabular}{|c|c|c|}
\hline Inverter systems & $\begin{array}{l}\text { Selected devices } \\
\text { (Number of pieces) }\end{array}$ & Total price \\
\hline PWM inverter & PM400DSA060, 600V/400A dual pack (3) & $\begin{array}{l}\$ 269.60 * 3 \\
=\$ \mathbf{8 0 8 . 8 0}\end{array}$ \\
\hline $\begin{array}{l}\text { PWM plus boost } \\
\mathrm{dc} / \mathrm{dc}\end{array}$ & $\begin{array}{l}\text { PM400DSA060, 600V/400A dual pack (1) plus } \\
\text { PM200CL060, 600V/200A } 6 \text { pack (1) }\end{array}$ & $\begin{array}{l}\$ 240+\$ 269.60 \\
=\$ \mathbf{5 0 9 . 6 0}\end{array}$ \\
\hline Z-source inverter & PM300CL060, 600V/300A 6 pack (1) & $\$ 308.88$ \\
\hline
\end{tabular}


Table 3. Required passive components

\begin{tabular}{|l|c|c|c|c|c|c|}
\hline \multicolumn{1}{|c|}{$\begin{array}{c}\text { Inverter } \\
\text { systems }\end{array}$} & $\begin{array}{c}\text { Number of } \\
\text { inductors }\end{array}$ & $\begin{array}{c}\text { Inductance } \\
(\mu \mathrm{H})\end{array}$ & $\begin{array}{c}\text { Average } \\
\text { inductor } \\
\text { current }(\mathrm{A})\end{array}$ & $\begin{array}{c}\text { Number of } \\
\text { capacitors }\end{array}$ & $\begin{array}{c}\text { Capacitance } \\
(\mu \mathrm{F})\end{array}$ & $\begin{array}{c}\text { Capacitor } \\
\text { rms ripple } \\
\text { current }(\mathrm{A})\end{array}$ \\
\hline $\begin{array}{l}\text { Conventional } \\
\text { PWM } \\
\text { inverter }\end{array}$ & 0 & N/A & N/A & 1 & 667 & 106 \\
\hline $\begin{array}{l}\text { dc/dc boost } \\
+ \text { PWM }\end{array}$ & 1 & 510 & 200 & 1 & 556 & 124 \\
\hline $\begin{array}{l}\text { Z-source } \\
\text { inverter }\end{array}$ & $2(1)$ & 384 & 200 & 2 & 420 & 115 \\
\hline
\end{tabular}

Switching devices were selected for each inverter topology to calculate their losses. The switches for the main inverters were FUJI IPM 6MBP300RA060; the switch for the dc/dc boost converter was FUJI $2 \mathrm{MBI} 300 \mathrm{~N}-060$. The operating conditions are listed in Table 4. The calculated efficiencies of inverters, as well as of the inverter plus the motor, are shown in Figures 4 and 5, respectively. Based on this simulated comparison, the Z-source inverter provides the highest efficiency.

Table 4. Operating conditions at different power levels

\begin{tabular}{|c|c|c|c|c|c|c|}
\hline \multicolumn{2}{|c|}{ Power rating } & $\begin{array}{c}50 \mathrm{~kW} \\
56 \mathrm{kVA}\end{array}$ & $\begin{array}{c}40 \mathrm{~kW} \\
47 \mathrm{kVA}\end{array}$ & $\begin{array}{c}30 \mathrm{~kW} \\
38 \mathrm{kVA}\end{array}$ & $\begin{array}{c}20 \mathrm{~kW} \\
27 \mathrm{kVA}\end{array}$ & $\begin{array}{c}10 \mathrm{~kW} \\
14 \mathrm{kVA}\end{array}$ \\
\hline \multirow{2}{*}{ Fuel cell voltage (V) } & 250 & 280 & 305 & 325 & 340 \\
\hline \multirow{2}{*}{ Motor current (A) } & Conventional PWM inverter & 209.4 & 158.5 & 115.9 & 77.3 & 39.7 \\
\cline { 2 - 7 } & dc/dc boost +PWM inverter & 124.7 & 105.6 & 84.2 & 59.9 & 32.1 \\
\cline { 2 - 7 } & Z-source inverter & 129.5 & 105.3 & 81.1 & 56.2 & 29.6 \\
\hline
\end{tabular}

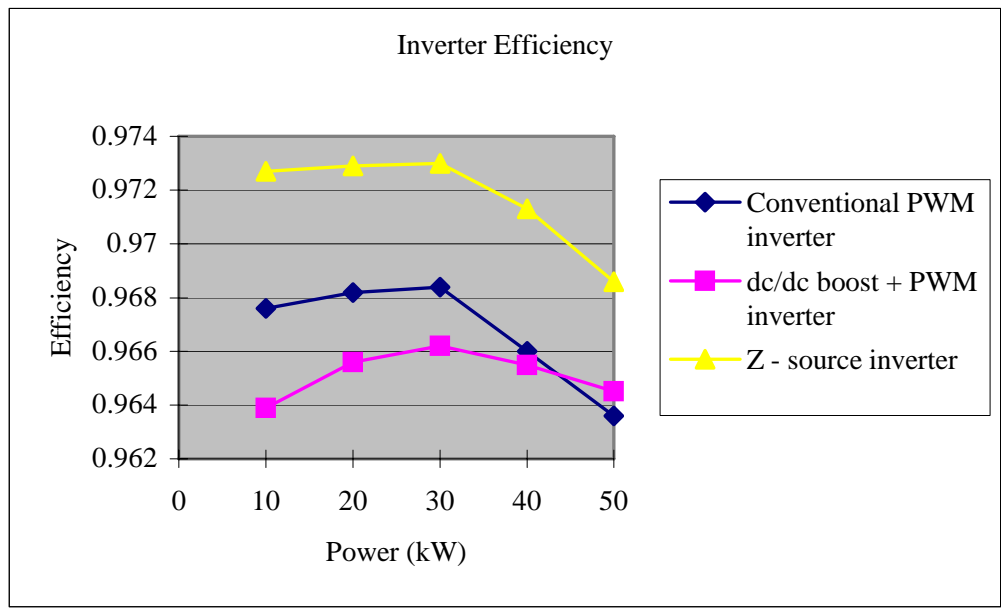

Figure 4. Calculated efficiency of inverters. 


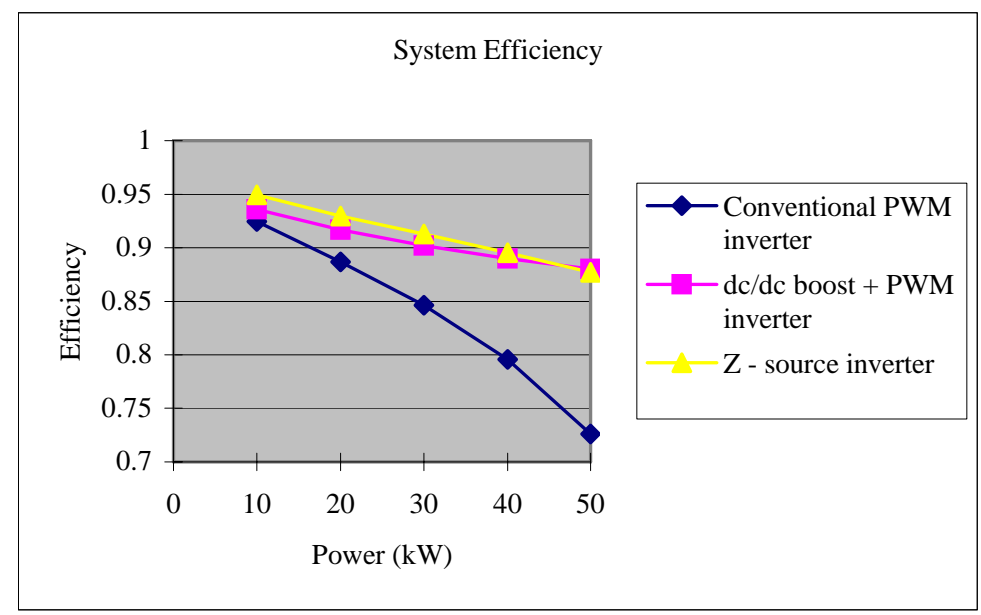

Figure 5. Calculated efficiency of inverters plus motor.

The comprehensive comparison results show that the Z-source inverter can increase inverter conversion efficiency by $1 \%$ over the two existing systems and inverter-motor system efficiency by 2 to $15 \%$ over the conventional PWM inverter. The Z-source also reduces the SDPR by $15 \%$, which leads to cost reduction. Moreover, the CPSR is greatly extended (1.55 times) compared with the system driven by the conventional PWM inverter. Thus the Z-source inverter system can minimize stresses and motor size and increase output power greatly. Along with these promising results, the Z-source inverter offers a simplified single-stage power conversion topology and higher reliability because shoot-through can no longer destroy the inverter. The existing two inverter systems suffer the shoot-through reliability problem. In summary, the Z-source inverter is very promising for this application.

A prototype was developed at MSU and was shipped to ORNL after initial evaluation and troubleshooting. Figure 6 is a photograph of the completed prototype.

The inverter efficiency was measured during the test at ORNL. There were two types of loads-an RL load and a dynamometer. For the RL load in normal operation mode without boost, the modulation index of the inverter is kept constant, and the output voltage increases with the increase of the input voltage. During boost mode, the output voltage is controlled by a potentiometer controlling the modulation index and shoot-through duty ratio. Figure 7(a) shows the measured efficiency with the RL

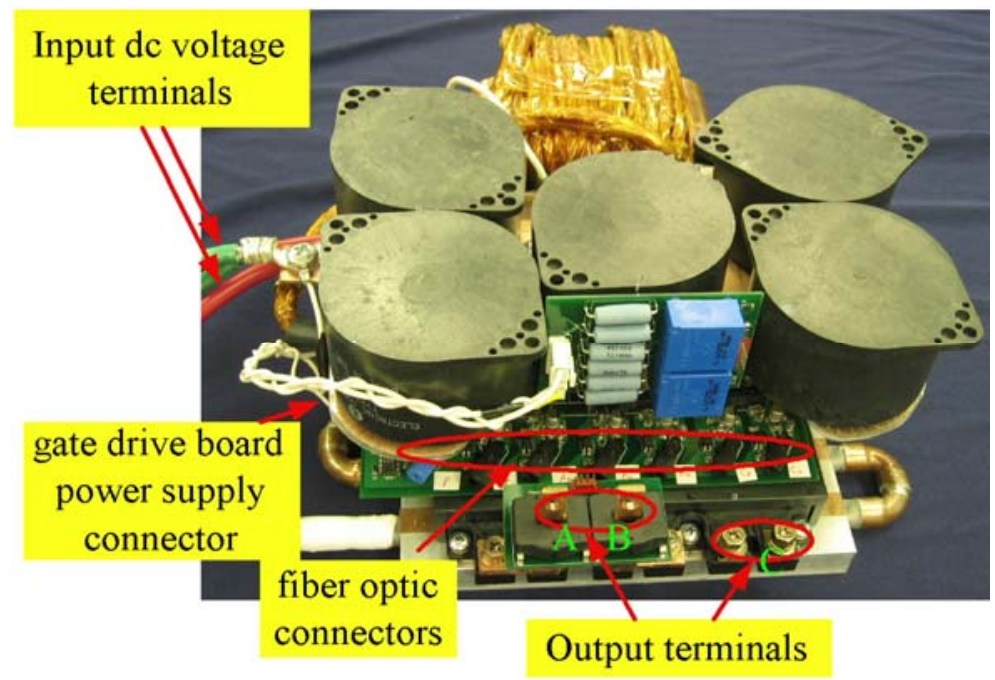

Figure 6. Final Z-source inverter prototype as tested. 
load. The point marked with a dark cross is the test point during boost; others are all during normal mode without shoot-through. For the test using the dynamometer as the load, the output voltage is always kept proportional to the output frequency to keep the magnetizing current constant (or constant V/F control); thus, the output power can be changed by adjusting the load torque, adjusting the input voltage, or adjusting the boost factor during boost mode. The inverter efficiency with different input voltages and different power levels is shown in Figure 7(b). Note that the inverter efficiency is defined against the output apparent power of the inverter. The points with dark marks are the testing points with boost, and the others are in normal operation modes without shoot-through. The efficiency was above $97 \%$ for most operation points, which achieved the $97 \%$ efficiency goal. More noticeably, the efficiency became much higher than $97 \%$ at medium and low power, which is extremely beneficial to vehicles, according to most driving cycles.

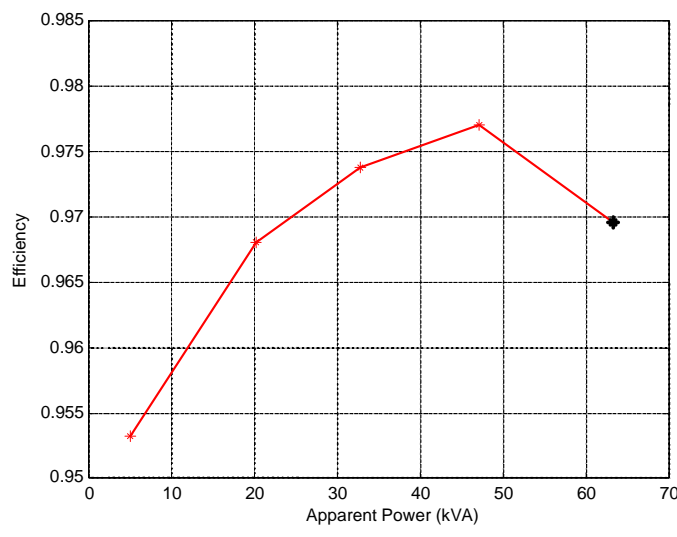

(a) Inductive load

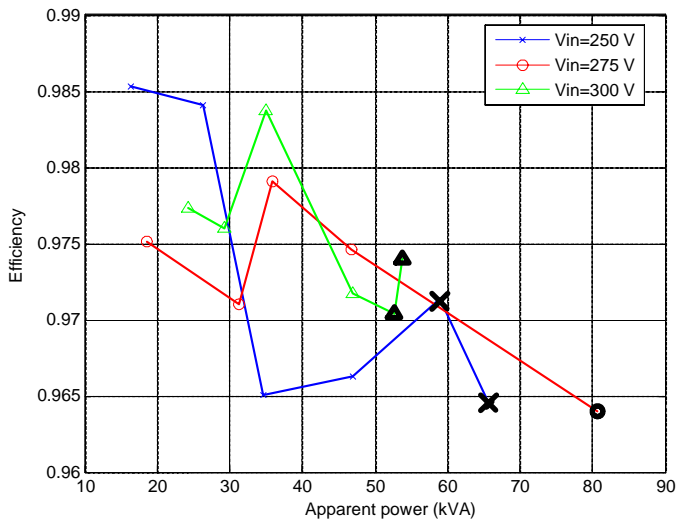

(b) Dyno load

Figure 7. Measured inverter efficiency.

FreedomCAR has a set of specifications and goals for the traction drive inverter to meet. However, it should be noted that the FreedomCAR has not yet set up clear goals for the dc-dc boost converter that are used for FCVs and HEVs. The Z-source inverter prototype has both inverter and dc-dc boost converter functions (i.e., equivalent to the traditional two stages of power conversion: inverter plus dc-dc boost converter), thus making comparison difficult. A comparison of the prototype to the initial goals of FreedomCAR is shown in Table 5. The numbers in the parentheses of the "FreedomCAR goals" column are the corresponding goals for the combined total system of inverter and dc-dc boost converter, assuming that the goals for the dc-dc boost converter are the same as the inverter. The power density numbers in the parentheses of the "Prototype" column are based on the actual peak power capacity of $80 \mathrm{~kW}$ that was successfully tested at ORNL, while the outside numbers are based on the design value of $55 \mathrm{~kW}$.

As can be seen from the table, the most important goals - the efficiency, power, and current ratingsare all well met and exceeded. The power density goals are well met by the prototype based on the actual peak power capacity of $80 \mathrm{~kW}$ for the combined dc-dc boost converter and inverter system goals. The only problem is the coolant temperature goal of $105^{\circ} \mathrm{C}$, which was not possible for this prototype with the semiconductor device's maximum junction temperature limit of $125^{\circ} \mathrm{C}$. Future higher-temperature devices and more advanced circuit topology may make this goal possible. A commercially available heatsink is used in the prototype for cost consideration, which contributes almost half of the inverter weight and a significant portion of the inverter volume. The capacitors used are commercial products, which have a cylinder shape with legs that are difficult for packaging. Significant void space exists in the prototype, thus making the volume unnecessarily larger. The size and the weight of the prototype can be further reduced with custom-designed heatsink and capacitor shapes. Once again, all the goals (efficiency, 
Table 5. Comparison of the prototype and the FreedomCAR goals

\begin{tabular}{|l|c|c|}
\hline Requirement & $\begin{array}{c}\text { FreedomCAR goals } \\
\text { Inverter (inverter+dc-dc) }\end{array}$ & Prototype \\
\hline Specific power @ peak load (kW/kg) & $>12(6)^{*}$ & $4.7(6.8)^{\dagger}$ \\
\hline Volumetric power density (kW/1) & $>12()^{*}$ & $4.7(6.8)^{\dagger}$ \\
\hline Efficiency, 10-100\% speed, 20\% rated torque (\%) & $>97(93)^{*}$ & $>97 \%$ \\
\hline Peak power (kW) & $55(5)^{*}$ & 30 \\
\hline Continuous power (kW) & $30(3)^{*}$ & 300 \\
\hline Maximum current, rms (A) & $300(30)^{*}$ & 77 \\
\hline Coolant inlet temperature & $105(10)^{*}$ & 300 \\
\hline
\end{tabular}

* Indicates the goals for the total combined system of inverter and dc-dc boost converter assuming the dc-dc boost converter has the same goals as the inverter.

$\dagger$ Shows the prototype power density values based on the actual peak power capacity of $80 \mathrm{~kW}$ that was tested, while the value of 4.7 is based on the designed peak power of $55 \mathrm{~kW}$.

power, and current) that are related to the technology are well met and exceeded. The volume and weight goals that are strongly related to engineering aspects can be met through better engineering and customdesigned parts for full utilization of the space.

\section{Conclusion}

This report has summarized the results and findings of the work conducted on the Z-source inverter for FCVs. The major work completed in this project includes (1) development of a low-cost, highefficiency traction drive Z-source inverter for FCVs; (2) a detailed comparison of the Z-source inverter with traditional inverter topologies; (3) the design and fabrication of a 55-kW Z-source inverter; and (4) testing and demonstration of the Z-source inverter prototype.

First, a unique Z-source inverter was developed for FCVs. This inverter is especially suited for a fuel cell that requires unidirectional current/power flow and has a wide voltage range. At present, there are two existing topologies for FCVs: the traditional PWM inverter and the dc/dc-boosted PWM inverter. The traditional PWM inverter topology uses the fuel cell voltage directly, which results in a limited CPSR for the motor and a freeze-start problem for the fuel cell. The dc/dc-boosted PWM inverter topology suffers from high cost, low converter efficiency, and complexity. The specially developed Z-source inverter can solve these problems.

Second, a comprehensive comparison was conducted of the Z-source inverter and the two existing inverter topologies mentioned. The comparison results showed that the Z-source inverter is highly suitable for FCVs, having a lower cost (20\% lower semiconductor ratings), higher conversion efficiency (1\% higher), a wider CPSR (1.68 times that of the traditional PWM inverter), and greater reliability (no need for dead time).

A 55-kW Z-source inverter was designed and fabricated. The detailed design process included circuit design, 3-D design, and thermal design. To reduce the switching loss of the inverter, a new PWM scheme for shoot-through was introduced.

Finally, lab testing was conducted at MSU and ORNL separately. Testing results confirmed the theoretical analysis and comparison results obtained and showed that the inverter had higher efficiency (up to more than 98\%) and low harmonic distortion (i.e., low current and torque ripples).

A self-boost phenomenon of the Z-source inverter when the traction motor was operating at low speed, low power, and low power factor was observed during the test. This self-boost function was initially deemed a problem; however, it turned out to be a needed function for successful fuel cell startup, especially for freeze-starts. In addition, this self-boost can be totally controllable when a battery is incorporated into the inverter system. A detailed analysis of the self-boost phenomenon and a control method using a battery was provided and confirmed by simulation.

In summary, the results of the project have shown high feasibility and many unique features of Zsource inverter for FCVs. 


\section{Future Direction}

This project is complete. Follow-on work has been recommended to address the issues and possibilities of using the Z-source inverter in more near-term bridging technologies such as in more conventional HEVs with a combination of an internal combustion engine and batteries rather than a fuel cell.

\section{$\underline{\text { Publications }}$}

Fang Z. Peng, "Z-Source Inverter," pp.775-781 in Proc. of IEEE IAS 2002.

Fang Z. Peng, "Z-Source Inverter," IEEE Transactions on Industry Applications, 39(2), March-April 2003, pp. 504-510.

Fang Z. Peng, Xiaoming Yuan, Xupeng Fang, and Zhaoming Qian, "Z-Source Inverter for Adjustable Speed Drives," IEEE Power Electronics Letters, 1(2), June 2003, pp. 33-35.

Fang Z. Peng, "Z-Source Inverter for Adjustable Speed Drives," IEEE/PESC Aachen, 2004, pp. 249-254.

Fang Z. Peng, Miaosen Shen, and Zhaoming Qian, "Maximum Boost Control of the Z-Source Inverter," IEEE/PESC Aachen, 2004, pp. 255-260.

Xupeng Fang, Zhaoming Qian, Qi Gao, Bin Gu, Fang. Z. Peng, and Xiaoming Yuan, "Current Mode Z-Source Inverter-Fed ASD system,” IEEE/PESC Aachen, 2004, pp. 2805-2809.

Miaosen Shen, Jin Wang, Alan Joseph, Fang Z. Peng, Leon M. Tolbert, and Donald J. Adams, "Maximum Constant Boost Control of the Z-Source Inverter," IEEE/IAS, Seattle 2004, pp. 142-147. Miaosen Shen, Alan Joseph, Jin Wang, Fang Z. Peng, and Donald J. Adams, "Comparison of Traditional Inverter and Z-Source Inverter for Fuel Cell Vehicles," IEEE WPET 2004, pp. 125-132.

Fang Z. Peng, "Z-Source Converters and Their Motor Drive Applications," International Conference on Electric Machines and Systems, invited paper, no. 630-M08-052, Jeju, Korea, Nov. 1-3, 2004.

F. Z. Peng, M. Shen, and A. Joseph, "Z-Source Inverters, Controls, and Motor Drive Applications," KIEE International Transactions on Electrical Machinery and Energy Conversion Systems, vol. 5-B 2005, pp. 6-12.

Yi Huang, Miaosen Shen, and Fang Z. Peng, “A Z-source Inverter for Residential Photovoltaic Systems," International Power Electronics Conference, Niigata, Japan, 2005.

Fang Z. Peng, Alan Joseph, Jin Wang, Miaosen Shen, Lihua Chen, Zhiguo Pan, Eduardo Ortiz, and Yi Huang, "Z-Source Inverter for Motor Drives," to be published in IEEE Transactions on Power Electronics, 20(4), 2005.

Fang Z. Peng, Miaosen Shen, and Zhaoming Qian, "Maximum Boost Control of the Z-Source Inverter," to be published in IEEE transactions on Power Electronics, 20(4), 2005.

Miaosen Shen, Alan Joseph, Jin Wang, Fang Z. Peng, and Donald J. Adams, "Comparison of Traditional Inverters and Z-Source Inverter," to be presented at IEEE PESC 2005.

Miaosen Shen, Fang Z. Peng, "Study of the Z-Source Inverter," to be presented at IEEE IAS 2005.

Kent Holland, Miaosen Shen, and Fang Z. Peng, "Z-Source Inverter Control for Traction Drive of Fuel Cell-Battery Hybrid Vehicles," to be presented at IEEE IAS 2005.

Kent Holland and Fang Z. Peng, "Control Strategy for Fuel Cell Vehicle Traction Drive Systems Using the Z-Source Inverter," to be presented at IEEE VPPC 2005.

Yi Huang, Miaosen Shen, and Fang Z. Peng, "A Z-Source Inverter for Residential Photovoltaic Systems," submitted to IEEE Transactions on Power Electronics.

Miaosen Shen, Jin Wang, Alan Joseph, Fang Z. Peng, Leon M. Tolbert, and Donald J. Adams, "Constant Boost Control of the Z-Source Inverter to Minimize Current Ripple and Voltage Stress," submitted to IEEE Transactions on Industry Applications.

Xu Peng Fang, Zhao Ming Qian, Qi Gao, Bin Gu, Miaosen Shen, and Fang Zheng Peng, "Z Source PWM AC/AC Converters," submitted to IEEE Power Electronics Letters. 


\section{Patents}

The Z-Source converter intellectual property pre-dates this project and is owned by MSU. The patent application was filed on June 10, 2003, assigned application number 10/458,564, and is titled "Impedance Source Power Converter."

\section{References}

1. F. Z. Peng, Li Hui, Su Gui-Jia, and J. S. Lawler, "A New ZVS Bidirectional DC-DC Converter for Fuel Cell and Battery Application," IEEE Transactions on Power Electronics, 19(1), Jan. 2004, pp. 54-65.

2. S. E. Gay, Gao Hongwei, and M. Ehsani, "Fuel Cell Hybrid Drive Train Configurations and Motor Drive Selection," presented at Vehicular Technology Conference, 2002, 2(24-28), Sept. 2002, pp. 007-1010.

3. Tadaichi Matsumoto, Nobuo Watanabe, Hiroshi Sugiura, and Tetsuhiro Ishikawa, "Development of Fuel-Cell Hybrid Vehicle," presented at Fuel Cell Power for Transportation 2002 Conference, SAE 2002 World Congress, March 2000, ref: 2002-01-0096.

4. B. D'Souza, H. Rawlins, J. Machuca, C. Larson, M. Shuck, B. Shaffer, T. Maxwell, M. Parten, D. Vines, and J. Jones, "Texas Tech University Develops Fuel Cell Powered Hybrid Electric Vehicle for FutureCar Challenge 1998," presented at International Congress \& Exposition, March 1999, Detroit, MI, SAE 1999, Ref: 1999-01-0612.

5. J. Adams, W. Yang, K. Oglesby, and K. Osborne, "The Development of Ford's P2000 Fuel Cell Vehicle," presented at Society of Automotive Engineers 2000 World Congress, March 2000, Detroit, MI, SAE 2000, Ref: 2000-01-1061.

6. D. Tran and M. Cummins, "Development of the Jeep Commander 2 Fuel Cell Hybrid Electric Vehicle," presented at Future Transportation Technology Conference \& Exposition, August 2001, Costa Mesa, CA, SAE 2001, Ref: 2001-01-2508.

7. Kent Holland, "Z-Source Inverter Control for Traction Drive of Fuel Cell-Battery Hybrid Vehicles," Masters Thesis, Michigan State University, August 2005.

8. F. Z. Peng, "Z-Source Inverter," IEEE Transactions on Industry Applications, 39(2), pp. 504-510, March/April 2003.

9. Kent Holland, Miaosen Shen, and Fang Z. Peng, "Z-Source Inverter Control for Traction Drive of Fuel Cell—Battery Hybrid Vehicles," in Proceedings of IEEE IAS, 2005.

10. Miaosen Shen, Jin Wang, Alan Joseph, Fang Z. Peng, Leon M. Tolbert, and Donald J. Adams, "Maximum Constant Boost Control of the Z source Inverter," in Proceedings of IEEE IAS '04, 2004.

11. "General Considerations: IGBT \& IPM Modules," Powerex Application Notes for IPM PM600CLA060, A10-A27, Powerex Corporation.

12. Fang Z. Peng, Miaosen Shen, Zhaoming Qian, "Maximum Boost Control of the Z-Source Inverter," IEEE Transactions on Power Electronics, 20(4), July 2005. 


\title{
4.5 dc-dc Converter for Fuel Cell and Hybrid Vehicle
}

\author{
Principal Investigator: Laura Marlino \\ Oak Ridge National Laboratory \\ National Transportation Research Center \\ 2360 Cherahala Boulevard \\ Knoxville, TN 37932 \\ Voice: 865-946-1245; Fax: 865-946-1262; E-mail: marlinold@ornl.gov
}

DOE Technology Development Manager: Susan A. Rogers

Voice:202-586-8997; Fax:202-586-1600; E-mail: susan.rogers@ee.doe.gov

ORNL Program Manager:Mitch Olszewski

Voice:865-946-1350; Fax: 865-946-1262; E-mail: olszewskim@ornl.gov

\section{Objectives}

- Develop and fabricate a 5-kW dc-dc converter with a baseline $14-\mathrm{V}$ output capability for fuel cell and - hybrid vehicles to satisfy the following requirements:

- Higher efficiency (92\%)

- High coolant temperature capability $\left(105^{\circ} \mathrm{C}\right)$

— High reliability (15 years/150,000miles)

- Smaller volume (5 1)

- Lower weight $(6 \mathrm{~kg})$

— Lower cost $(\$ 75 / \mathrm{kW})$

\section{Approach}

The key technical challenge for these converters is the $105^{\circ} \mathrm{C}$ coolant temperature requirement. The power switches and magnetics must be designed to sustain high operating temperatures reliably, without a large cost/mass/volume penalty. During FY 2005, a 5-kW prototype was developed by Ballard Power Systems and initial testing was performed.

The following key technologies are being used to break through technical barriers to achieve a hightemperature, high-power-density, and lower-cost design.

\section{Converter topology}

A novel interleaved dc-dc converter topology (Figure 1) was proposed for this high power conversion. The key merits of the converter are

- Lower RMS current stresses on components due to interleaving

- Reduced ripple current on capacitors due to interleaving

- Lower power losses due to low Rds_on and soft-switching

- Smaller magnetics due to high switching frequency

- Low electromagnetic interference as a result of integrated power devices and magnetics

\section{Integrated module-based dc-dc converter}

The project uses Ballard's Class 10,000 clean-room power semiconductor packaging facility to develop and apply custom packaging processes and materials that address size, heat, and inductancerelated burdens while reducing component cost. The custom packaging process reduces size by increasing the density of the electronic assemblies. Custom packaging materials will lower heat generation by reducing thermal interface layers and facilitating the integration of advanced cooling methods. 


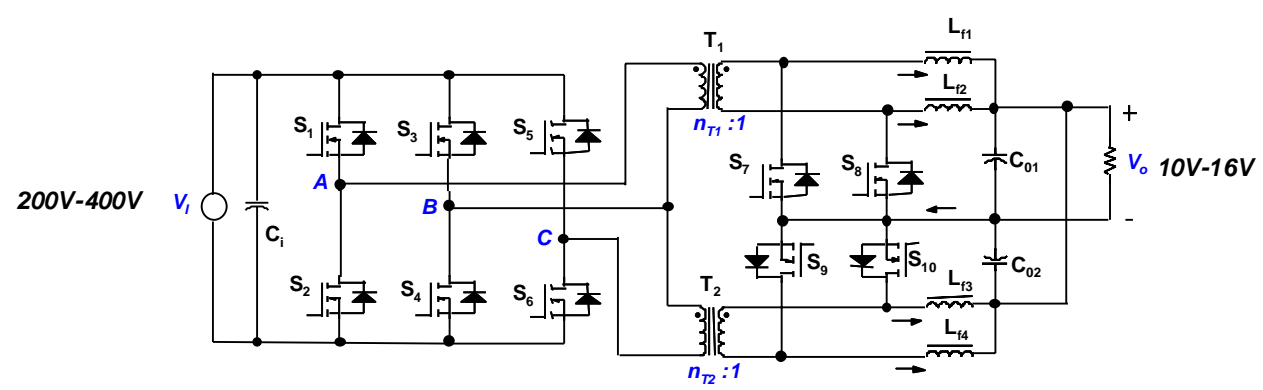

Figure 1. The novel interleaved dc-dc converter topology.

The major advantages of the power module based dc-dc converter are

- Enhanced thermal performance

- Reduced number of devices

- Increased reliability

- Higher level of integration

3. Planar magnetics with enhanced cooling

This converter has also been designed using planar magnetics, a technology that Ballard believes is critical for reliable and cost-effective high-volume production of such products. The benefits from this technology are

- Lower leakage inductance as a result of shorter winding termination and smaller circuit paths

- Elimination of discrete contacts' ohmic loss

- Reduction of ohmic loss as a result of shorter conduction paths

- Lower ac loss due to flat winding structure

- Higher core window utilization ratio

- Smaller core volume and weight

- Higher surface-to-volume ratio for improved heat conduction

- Direct cooling of core by direct contact to heat sink

- Higher power density

\section{Major Accomplishments}

The project is divided into four major phases: Phase 1: key technologies prove-out; Phase 2: fullfunction alpha design and test; Phase 3: final beta prototype design; Phase 4: final build, DV test, delivery and report. Ballard Power Systems has achieved the following during FY 2005.

1. Key technologies proved out

A $2.5-\mathrm{kW}$ segmented dc-dc power module was developed at the beginning of FY 2005 to verify key technologies adopted in this project. Figures 2, 3, and 4 show the preliminary power module and the test results. Through this first design iteration, the following technologies have been verified and implemented:

- $\quad$ DBC transformer winding design and manufacture feasibility

- Key variables affecting the performance

- Wirebond diagram and Rds_on packaging efficiency

- Non-interleave planar transformer vs interleaved planar transformer

- Metal oxide semiconductor field effect transistor die selection with low Qrr

- Snubber circuit optimization

- Transformer ratio

- Power module fabrication process with larger DBCs and lead frames 


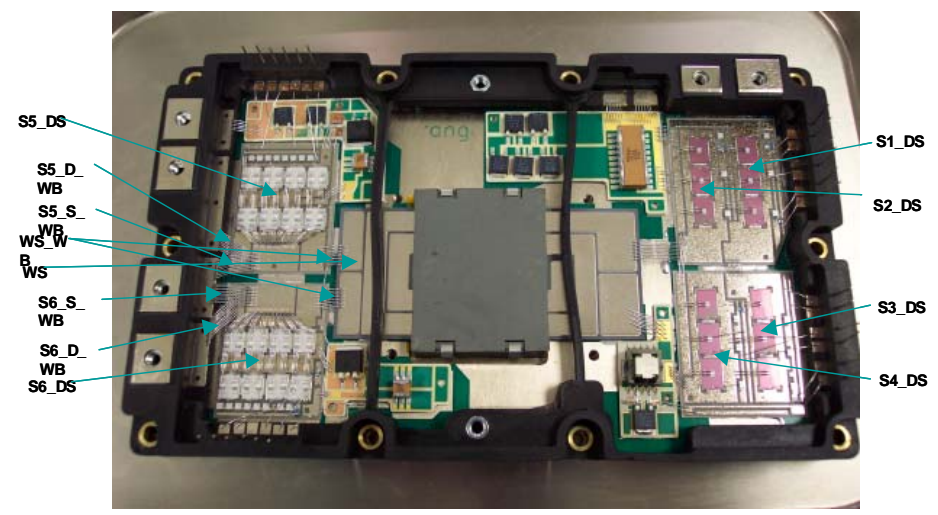

Figure 2. A segmented preliminary $2.5-\mathrm{kW}$ dc-dc power module.

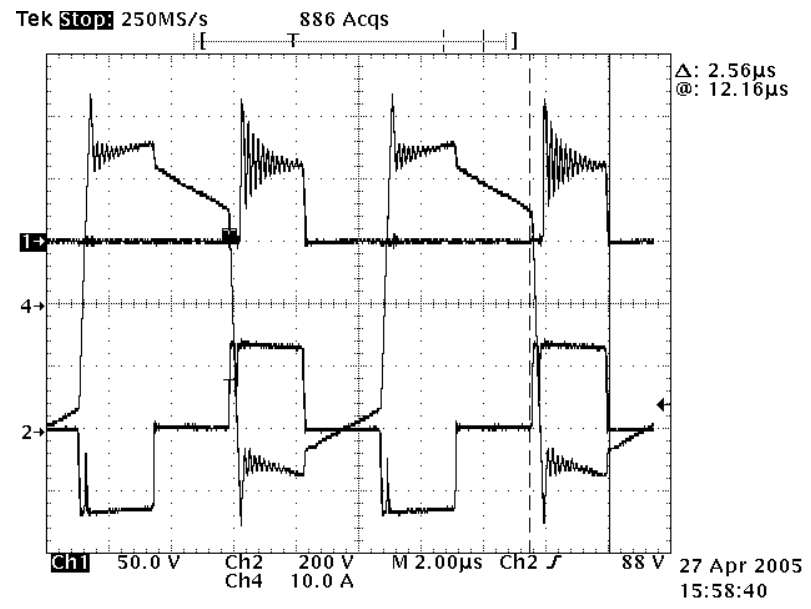

Figure 3. Tested waveforms at $\mathrm{Vi}=300 \mathrm{~V}, \mathrm{Vo}=13.35 \mathrm{~V} / 197 \mathrm{~A}, \mathrm{Po}=2627 \mathrm{~W}, \mathrm{I} \_r r=35 \mathrm{~A}$.

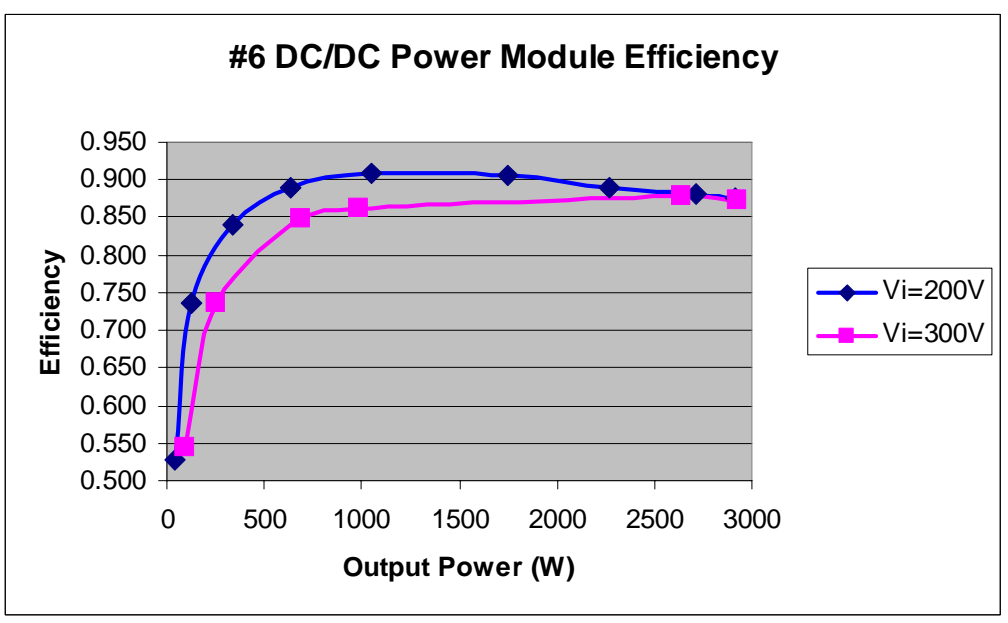

Figure 4. Efficiency mapping of the segmented dc-dc power module. 
2. Fully-functional, alpha unit prototype design and fabrication:

A 5-kW converter with the selected topology (Figure 1)was designed during this phase. The following major tasks were accomplished:

- Power module design, including DBC, lead frame, and module baseplate

- dc/dc converter packaging: housing plate, seal, busbar, etc

- Control board, erasable programmable logic device program, and simulation

- High-temperature components selection

- Design release and components purchasing

- Power module process development

- Fabrication and assembly

Figures 5 and 6 show the designed alpha $5-\mathrm{kW}$ power module and completed dc-dc converter. This design level incorporated the lesson learned from the $2.5-\mathrm{kW}$ segmented power module (described earlier), with the goal to verify full electrical function against the specification. To reduce prototyping time, the lead frame was made from urethane and the baseplate was made from aluminum. The final design will use plastic and AlSiC, respectively.

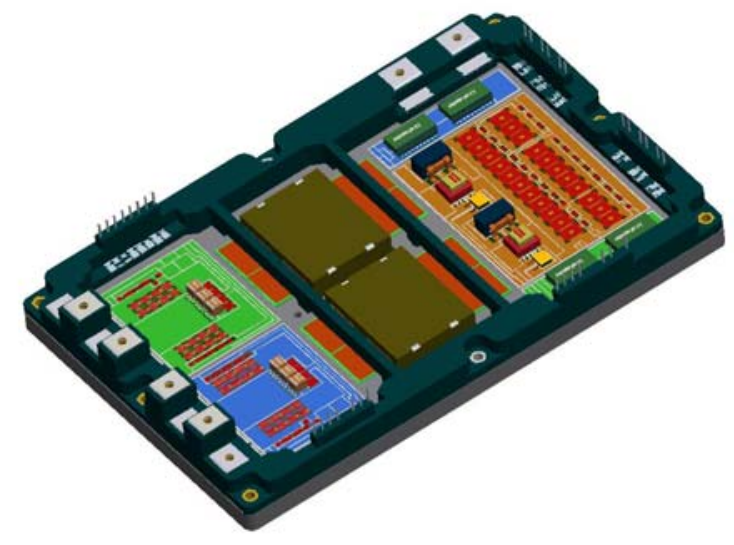

Figure 5. A 5-kW full functional dc-de power module-alpha design.

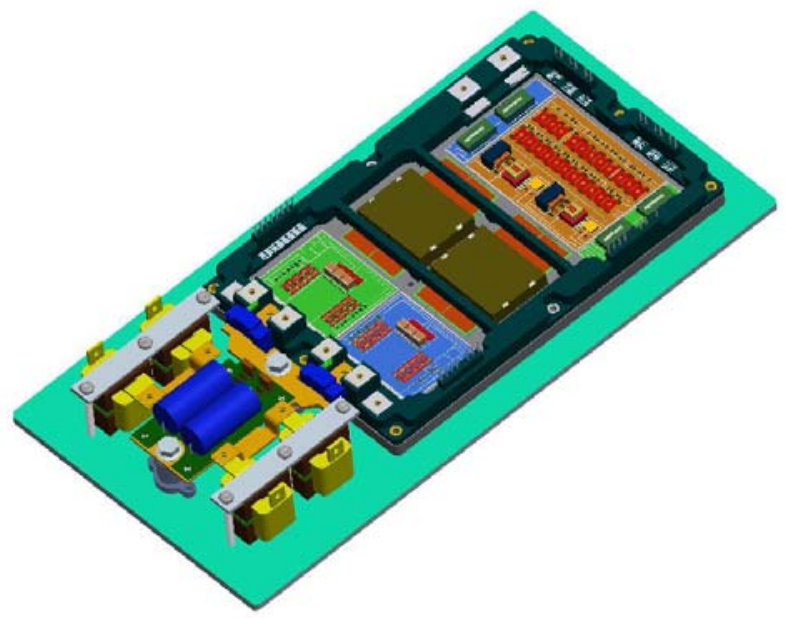

Figure 6. A completed 5-kW dc-dcconverter-alpha design. 


\section{High-volume cost estimation}

Based on the bill of material generated in the alpha version dc-dc converter design, a unit cost at high volume has been estimated, as illustrated in Table 1 . The current cost estimate for this module is \$545/per unit, $45 \%$ higher than the DOE target of $\$ 375 /$ per unit. The power module is the major cost in the dc-dc converter, composing 59\% of the total cost. Table 2 and Figure 7 contain a detailed study of the power module cost.

Table 1. Alpha dc-dc converter bill of material and cost estimation at $1000 \mathrm{~K}$ volume.

\begin{tabular}{|c|c|c|c|c|c|c|c|}
\hline$\#$ & Level & Part Description & Tooling Cost & Tooling Type & $\begin{array}{c}\text { Part Unit Price } \\
(@ 1 \mathrm{KK})\end{array}$ & \# Per Uint & Part Cost \\
\hline 1 & 1 & ORNL 5kW DC-DC Power Module & $\$ 166,000$ & & $\$ 320$ & 1 & $\$ 320$ \\
\hline 2 & 1 & LV Busbar Positive & $\$ 15,000$ & Stamping die & $\$ 2$ & 1 & $\$ 2$ \\
\hline 3 & 1 & LV Busbar Positive & $\$ 15,000$ & Stamping die & $\$ 2$ & 1 & $\$ 2$ \\
\hline 4 & 1 & Converter Housing & $\$ 80,000$ & Die cast & $\$ 15$ & 1 & $\$ 15$ \\
\hline 5 & 1 & Housing Cover & $\$ 50,000$ & Stamping die & $\$ 8$ & 1 & $\$ 8$ \\
\hline 6 & 1 & LV Studs & $\$ 30,000$ & Insert mold & $\$ 5$ & 2 & $\$ 10$ \\
\hline 7 & 1 & HV Connector & & & $\$ 20$ & 1 & $\$ 20$ \\
\hline 8 & 1 & Inductor & $\$ 10,000$ & & $\$ 9$ & 4 & $\$ 35$ \\
\hline 9 & 1 & LV Capacitor Board Assembly & & & $\$ 10$ & 1 & $\$ 10$ \\
\hline 10 & 1 & Signal Connector & & & $\$ 8$ & 1 & $\$ 8$ \\
\hline 11 & 1 & Control Board & 5000 & & $\$ 80$ & 1 & $\$ 80$ \\
\hline 12 & 1 & Current Sensor & & & $\$ 8$ & 2 & $\$ 16$ \\
\hline 13 & $x$ & MFG, F\&T & & & & & $\$ 18$ \\
\hline & & \multicolumn{2}{|c|}{ Tooling Cost } & & \multicolumn{2}{|c|}{ Part Cost Per Unit @1000K } & $\$ 545$ \\
\hline & & & & & \multicolumn{2}{|l|}{ DOE Target } & $\$ 375$ \\
\hline
\end{tabular}

Table 2. dc-dc power module cost spreadsheet

\begin{tabular}{|r|l|r|r|}
\hline 1 & Lead Frame & $\$ 10.00$ & $3 \%$ \\
\hline 2 & AlSic Baseplate & $\$ 50.00$ & $17 \%$ \\
\hline 3 & DBC Substrates & $\$ 57.86$ & $20 \%$ \\
\hline 4 & DBC XFMR Wingding & $\$ 36.50$ & $13 \%$ \\
\hline 5 & HV mosfets & $\$ 22.00$ & $8 \%$ \\
\hline 6 & LV mosfets & $\$ 32.00$ & $11 \%$ \\
\hline 7 & Chip Resistors & $\$ 10.80$ & $4 \%$ \\
\hline 8 & HV Caps & $\$ 32.00$ & $11 \%$ \\
\hline 9 & LV Caps & $\$ 12.00$ & $4 \%$ \\
\hline & Sub Total & $\$ 263.16$ & $90 \%$ \\
\cline { 2 - 4 } & Total Cost & $\$ 291.00$ & $100 \%$ \\
\hline
\end{tabular}

4. Alpha prototype test results

Figure 8 shows the ORNL alpha dc-dc converter test setup. The tests are still ongoing. Currently the converter attains $5.0-\mathrm{kW}$ output (full power) and achieves a peak efficiency of $95 \%$. From the test waveform shown in Figure 9, the Irr loss is eliminated compared with the segmented dc-dc converter tested earlier this year. The alpha unit efficiency map is shown in Figure 10. Comparing these results with the segmented dc-dc converter efficiencies found in Figure 4, overall efficiency in the alpha design has been improved by $5 \%$.

The measured volume of the alpha unit approaches $6.5 \mathrm{~L}$, approximately $1.5 \mathrm{~L}$ over the DOE target of $5 \mathrm{~L}$. The measured pressure drop of this module is approximately 2.3 psi, exceeding the DOE target by 1.57 psi. 


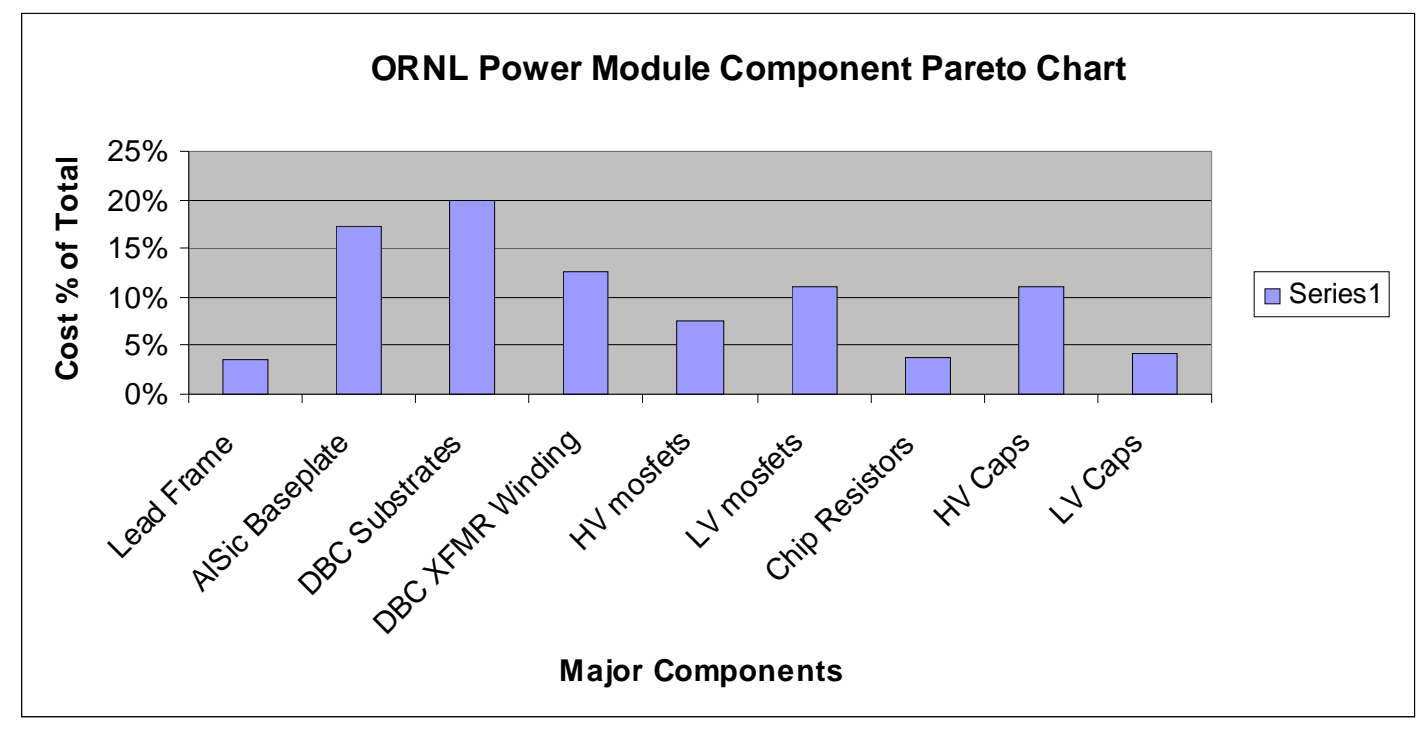

Figure 7. dc-dc power module cost distribution.

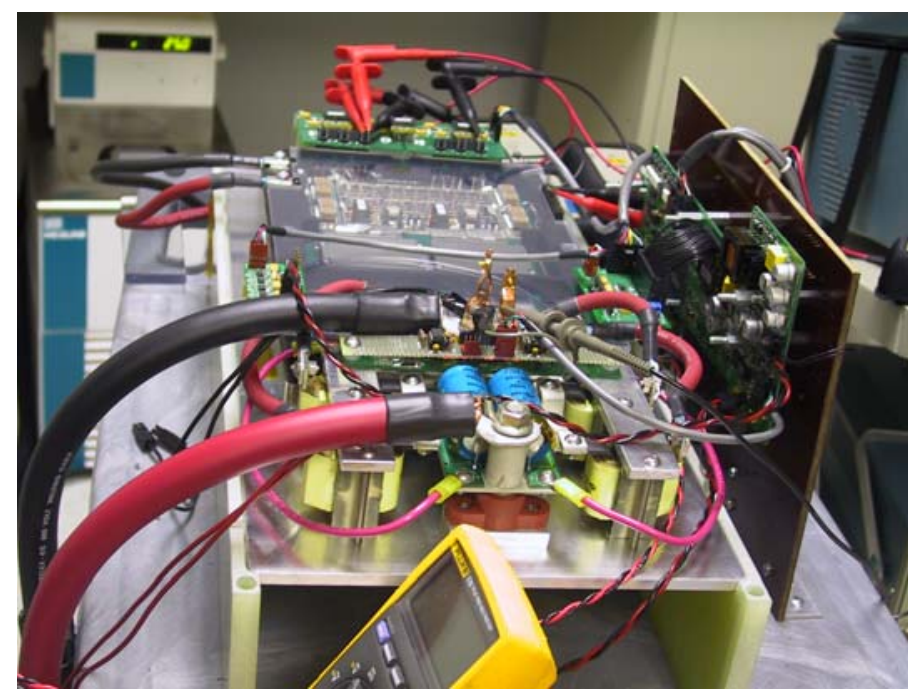

Figure 8. Alpha dc-dc converter under test. 


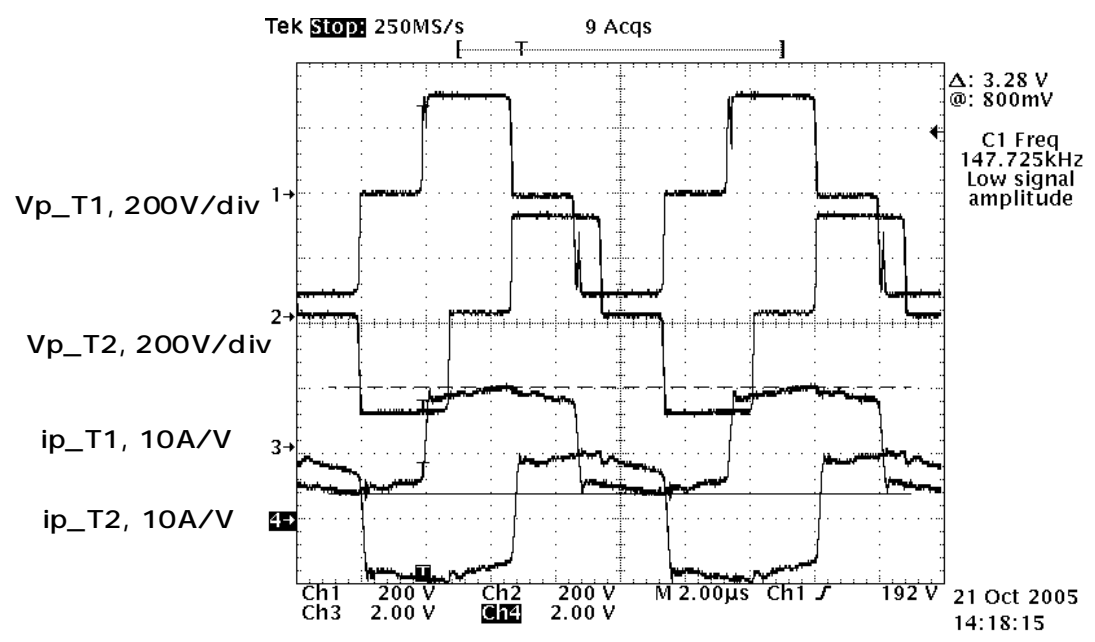

Figure 9. Alpha unit tested waveforms at $\mathrm{Vi}=300.6 \mathrm{~V}, \mathrm{Vo}=13.31 \mathrm{~V}, \mathrm{Po}=5.1 \mathrm{~kW}, \eta=93.3 \%$.

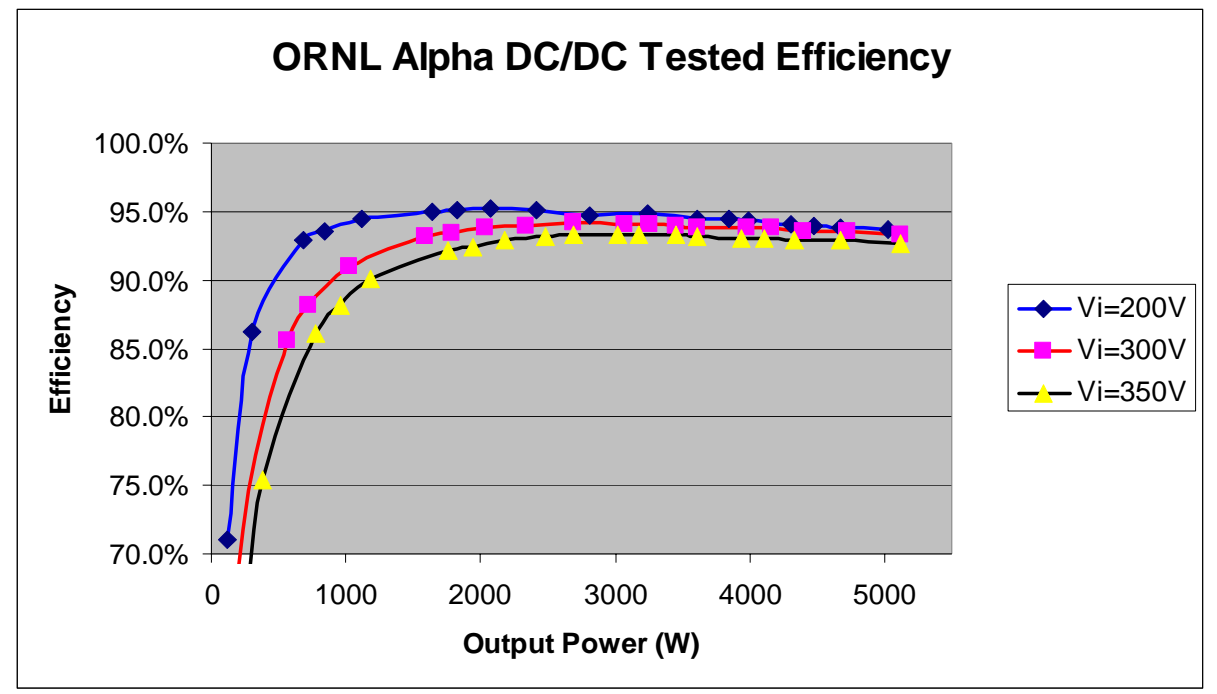

Figure 10. Alpha unit tested efficiency at $\mathrm{Vi}=200 \mathrm{~V}, 300 \mathrm{~V}, 350 \mathrm{~V}$ and up to $5 \mathrm{~kW}$.

\section{Technical Discussion}

The alpha-level prototype of the 5-kW dc-dc converter successfully verified the operation of the proposed topology and the improvement of the power module layout. The efficiency was improved by $5 \%$ compared with the preliminary design and achieved a peak efficiency of $95 \%$, exceeding the DOE target of $92 \%$. However, several issues require further improvement for the final beta level design:

- High-volume cost target must be met.

- Power density needs improvement, toward the DOE target.

- Large DBC size $(87 \times 112 \mathrm{~mm})$ exceeds the supplier's zone of comfort and has reliability implications (thermal cycling).

- Large lead frame results in excessive tooling costs.

- Large module baseplate approaches the limit of production facility capabilities.

- Pressure drop is higher than the target. 


\section{Conclusions}

- The key technologies have been proven.

- Alpha converter prototype has been designed.

- All components have been received and the system has been assembled.

- High-volume cost estimates have been developed.

- Testing has achieved full power output $(5 \mathrm{~kW})$.

- Efficiency has been improved by 5\% compared with the preliminary design.

- Volume and pressure drop of the alpha design have been tested.

- The up-to-date status from the alpha design is summarized in Table 3.

Table 3. Up-to-date status summary from the alpha design

\begin{tabular}{|l|l|l|l|}
\hline & Alpha Design & DOE Goal & $\%$ Target \\
\hline Output Power & $5.1 \mathrm{~kW}$ & $5 \mathrm{~kW}$ & $102 \%$ \\
\hline Efficiency & $93 \%$ & $92 \%$ & $101 \%$ \\
\hline Cost Estimation & $\$ 545$ & $\begin{array}{l}\$ 375 \text { total, } \\
(\$ 75 / \mathrm{kW})\end{array}$ & $69 \%$ \\
\hline $\begin{array}{l}\text { Coolant } \\
\text { Temperature }\end{array}$ & 90 Deg C & 105 Deg C & $86 \%$ \\
\hline Volume & $6.5 \mathrm{Liter}$ & $5 \mathrm{Liter}$ & $77 \%$ \\
\hline Weight & $7.6 \mathrm{~kg}$ & $6 \mathrm{~kg}$ & $79 \%$ \\
\hline $\begin{array}{l}\text { Coolant } \\
\text { Pressure Drop }\end{array}$ & $2.3 \mathrm{PSI}$ & $\begin{array}{l}0.73 \mathrm{PSI} \\
(5 \mathrm{kPa})\end{array}$ & $32 \%$ \\
\hline
\end{tabular}

\section{Future Direction}

This project will conclude in FY 2006. In order to finalize the beta level (final) design, the following steps will be completed during FY 2006:

- Perform beta design addressing gap areas toward the final targets.

- Reduce costs by further simplifying design and fabrication requirements.

- Reduce DBC size to improve reliability.

- Reduce module baseplate size to improve manufacturability.

- Reduce converter packaging volume.

- Reduce pressure drop through baseplate flow channel optimization.

- Develop/refine DV test plan.

\section{Patents}

“A Interleaved High Power DC/DC Converter," PAT0589-01US, filed June 4, 2004.

"Integration of Planar Magnetics Transformer and Power Switching Devices in a Liquid-cooled High Power DC/DC Converter," PAT0588-02US, filed October 12, 2004. 


\subsection{Semikron Automotive Integrated Power Module Testing}

Principal Investigator: Sam Nelson

Oak Ridge National Laboratory

National Transportation Research Center

2360 Cherahala Boulevard

Knoxville, TN 37932

Voice: 865-946-1327; Fax: 865-946-1262; E-mail:neslonsscjr@ornl.gov

DOE Technology Development Manager: Susan A. Rogers

Voice: 202-586-8997; Fax: 202-586-1600; E-mail: Susan.Rogers@ee.doe.gov

ORNL Program Manager: Mitch Olszewski

Voice: 865-946-1350; Fax: 865-946-1262; E-mail: olszewskim@ornl.gov

\section{$\underline{\text { Objectives }}$}

The objective of the Semikron Automotive Integrated Power Module (AIPM) testing task is to evaluate the electrical performance of the high-voltage Semikron AIPM. Electrical tests were performed on the unit at Oak Ridge National Laboratory (ORNL) during January, 2005. The objective of the electrical tests includes the determination of continuous power capability, peak power capability, and efficiency at various power levels.

\section{Approach}

The approach used in this task was to perform standard electrical tests on the Semikron unit. The unit was tested with an inductive load to evaluate its performance across a range of input voltages (i.e., minimal, nominal, and maximum), output power levels, and output frequencies. The unit was also tested while driving a load motor (Solectria motor) in a dynamometer test cell at the National Transportation Research Center (NTRC).

\section{Major Accomplishments}

The Semikron AIPM unit was successfully tested during January 2005 at ORNL. The Semikron AIPM inverter demonstrated the ability to operate at the continuous (i.e., 30-kW) and peak (i.e., 55-kW) power levels with an efficiency of 0.97 when supplied with $70^{\circ} \mathrm{C}$ coolant at $2.5 \mathrm{gpm}$ during induction load testing. The inverter operated at the continuous power level for $60 \mathrm{~min}$ during the induction load tests with an average efficiency of 0.972. During dynamometer testing, driving a load motor, the inverter had efficiencies that ranged from 0.94 to 0.97 when operated at continuous power levels.

\section{Technical Discussion}

The Semikron inverter was tested during January 2005 at ORNL. The initial testing of the inverter was performed in room L101 of the NTRC with an inductive/resistive load. The load was connected in a Y-arrangement with an inductor and a resistor connected in series for each phase leg. Figure 1 shows a top view of the Semikron AIPM unit being load tested. Figure 2 shows the notebook computer containing the controller area network (CAN) bus controller program, the Yokogawa power meter, and the power supply for the Semikron controller. The Semikron inverter is in the background in Figure 2. Figure 3 shows a more detailed view of the CAN bus display. 


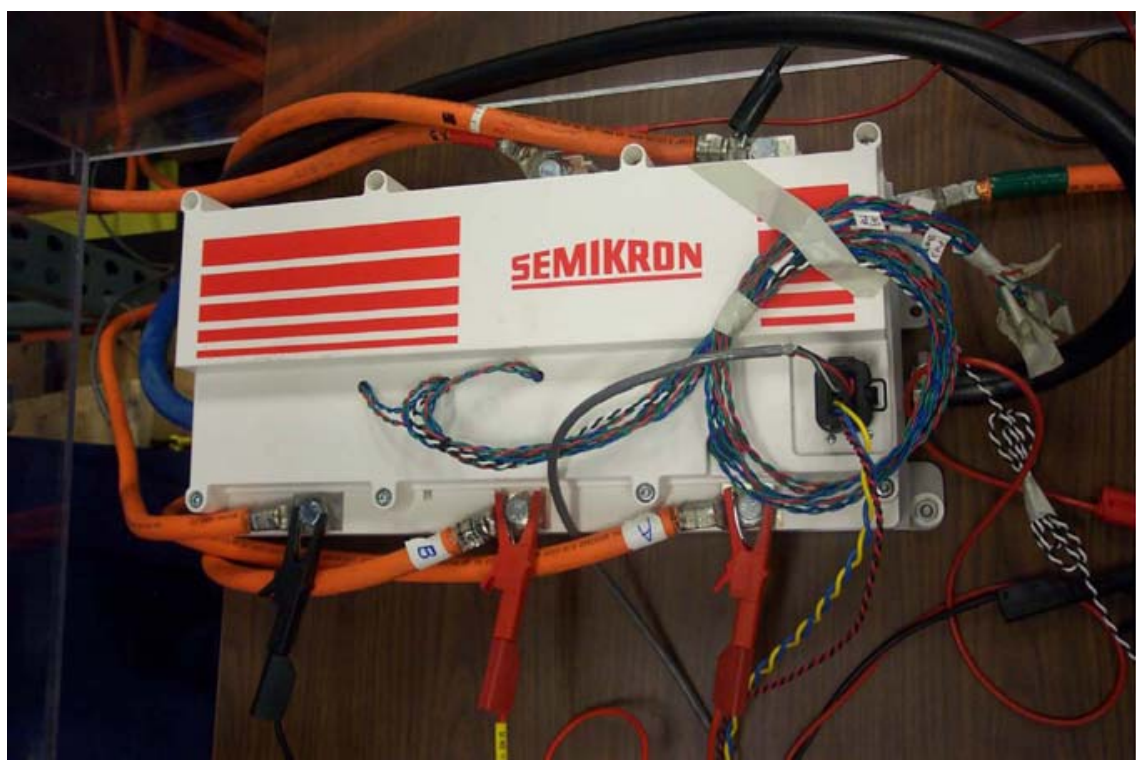

Figure 1. Top view of Semikron AIPM unit.

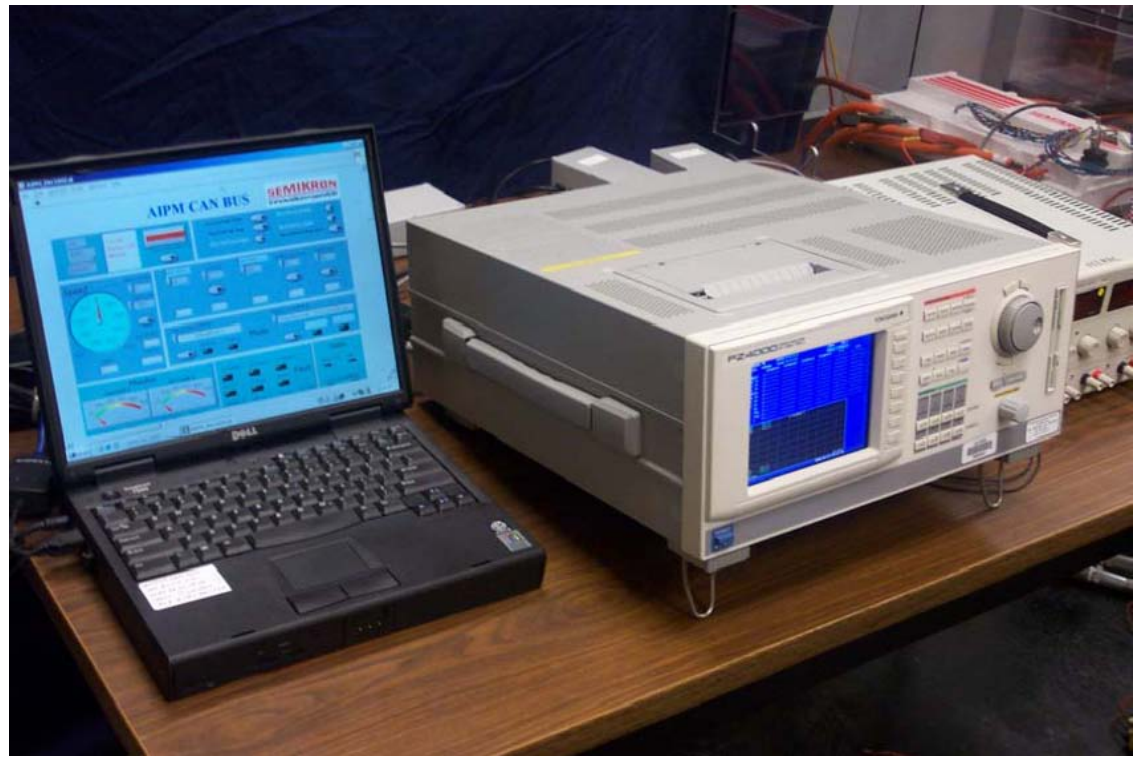

Figure 2. Control computer, power meter, power supply, and Semikron unit.

Table 1 lists the test results when the inverter was operated at $50 \mathrm{~Hz}$ at six different power levels with a $200-\mathrm{Vdc}$ link for short-duration testing. The unit was operated at each test point for about 1 min with $20^{\circ} \mathrm{C}$ coolant at a flow rate of $2.5 \mathrm{gpm}$. The average efficiency for the six data points is 0.966 , and the efficiency increased with increased power levels.

Table 2 displays the test results when the inverter was operated with a 250-Vdc link for short-duration testing with $20^{\circ} \mathrm{C}$ coolant at a 2.5 -gpm flow. The unit had an average efficiency of 0.962 when operated with an output frequency of $10 \mathrm{~Hz}$ at seven different power levels. The inverter had an average efficiency of 0.976 when operated at five different output frequencies (i.e., 10, 20,30,50, and $60 \mathrm{~Hz}$ ) with a requested dc link current of $80 \mathrm{~A}$. The average efficiency during the load testing with a $250-\mathrm{Vdc}$ link was 0.968 . 


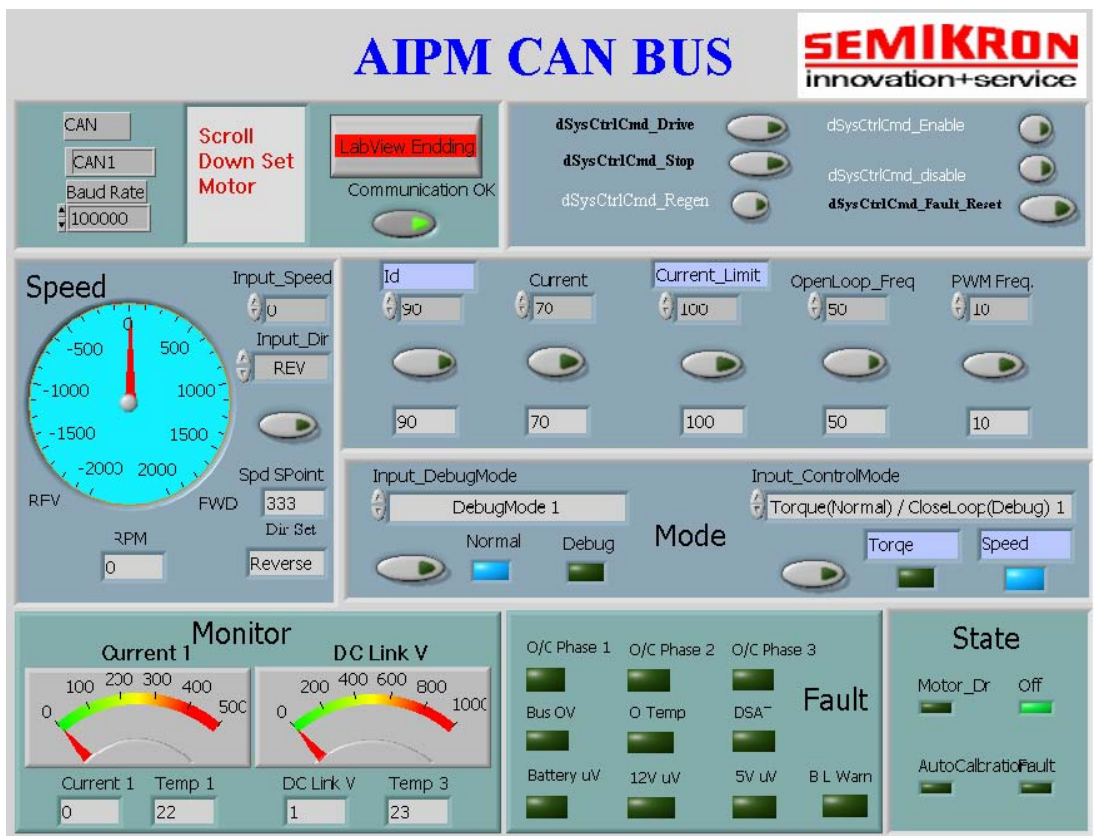

Figure 3. CAN bus display.

Table 1. Short-duration testing of Semikron inverter with 200-Vdc link

\begin{tabular}{cccccccccc}
\hline dc link & Freq & Idc & Vac & Iac & Pin & Pout & Effic. & Cool. & Flow \\
\hline$(\mathbf{V d c})$ & $(\mathbf{H z})$ & $(\mathbf{A})$ & $\left(\mathbf{V}_{\mathbf{L L}}\right)$ & $\left(\mathbf{A}_{\mathbf{r m s}}\right)$ & $(\mathbf{k W})$ & $(\mathbf{k W})$ & & $(\mathbf{C})$ & $(\mathbf{g p m})$ \\
200 & 50 & 7.5 & 87.5 & 24.9 & 1.51 & 1.43 & 0.947 & 20 & 2.5 \\
200 & 50 & 16.5 & 106.3 & 34.0 & 3.3 & 3.15 & 0.955 & 20 & 2.5 \\
200 & 50 & 28.1 & 123.1 & 43.3 & 5.71 & 5.54 & 0.970 & 20 & 2.5 \\
200 & 50 & 43.3 & 137.0 & 53.6 & 8.79 & 8.56 & 0.974 & 20 & 2.5 \\
200 & 50 & 61.5 & 149.4 & 64.5 & 12.46 & 12.14 & 0.974 & 20 & 2.5 \\
200 & 50 & 74.9 & 156.8 & 70.8 & 15.13 & 14.8 & 0.978 & 20 & 2.5 \\
\hline
\end{tabular}

Table 2. Short-duration testing of Semikron inverter with 250 -Vdc link

\begin{tabular}{cccccccccc}
\hline dc link & Freq & Idc & Vac & Iac & Pin & Pout & Effic. & Cool. & Flow \\
\hline$(\mathbf{V d c})$ & $(\mathbf{H z})$ & $(\mathbf{A})$ & $\left(\mathbf{V}_{\mathbf{L L}}\right)$ & $\left(\mathbf{A}_{\text {rms }}\right)$ & $(\mathbf{k W})$ & $(\mathbf{k W})$ & & $(\mathbf{C})$ & $(\mathbf{g p m})$ \\
250 & 10 & 6.09 & 94.18 & 22.7 & 1.53 & 1.44 & 0.941 & 20 & 2.5 \\
250 & 10 & 13.19 & 114 & 33.7 & 3.3 & 3.13 & 0.948 & 20 & 2.5 \\
250 & 10 & 22.99 & 131.3 & 44.4 & 5.75 & 5.54 & 0.963 & 20 & 2.5 \\
250 & 10 & 35.19 & 146 & 54.2 & 8.79 & 8.5 & 0.967 & 20 & 2.5 \\
250 & 10 & 50.32 & 159.4 & 64.0 & 12.56 & 12.21 & 0.972 & 20 & 2.5 \\
250 & 10 & 67.62 & 171.7 & 74.0 & 16.88 & 16.45 & 0.975 & 20 & 2.5 \\
250 & 10 & 89.51 & 183.6 & 84.8 & 22.35 & 21.61 & 0.967 & 20 & 2.5 \\
250 & 20 & 89.99 & 184.9 & 85.5 & 22.47 & 21.84 & 0.972 & 20 & 2.5 \\
250 & 30 & 90.7 & 107.5 & 86.4 & 22.66 & 22.28 & 0.983 & 20 & 2.5 \\
250 & 50 & 87.08 & 190.4 & 84.7 & 21.71 & 21.21 & 0.977 & 20 & 2.5 \\
250 & 60 & 86.56 & 194.6 & 84.7 & 21.67 & 21.26 & 0.981 & 20 & 2.5 \\
\hline
\end{tabular}


Table 3 shows the test results when the unit was operated with a 400- and 450-Vdc link for shortduration testing. With a $400-\mathrm{Vdc}$ link, the unit was operated with an output frequency of $10 \mathrm{and} 50 \mathrm{~Hz}$ with the same requested output current. The unit had a higher efficiency $(0.986)$ when operated at $50 \mathrm{~Hz}$. The unit was tested with a $450-\mathrm{Vdc}$ link with an output frequency of 10,50 , and $70 \mathrm{~Hz}$ and the same requested output current. The efficiency exceeded the efficiency goal (i.e., 0.97) at 50 and $70 \mathrm{~Hz}$ but was below specification at $10 \mathrm{~Hz}(0.939)$.

Table 3. Short-duration testing of Semikron inverter with 400- and 450-Vdc link

\begin{tabular}{cccccccccc}
\hline dc link & Freq & Idc & Vac & Iac & Pin & Pout & Effic. & Cool. & Flow \\
\hline$(\mathbf{V d c})$ & $(\mathbf{H z})$ & $(\mathbf{A})$ & $\left(\mathbf{V}_{\mathbf{L L}}\right)$ & $\left(\mathbf{A}_{\mathbf{r m s}}\right)$ & $(\mathbf{k W})$ & $(\mathbf{k W})$ & & $(\mathbf{C})$ & $(\mathbf{g p m})$ \\
400 & 10 & 35.6 & 294.7 & 37.7 & 14.22 & 13.95 & 0.981 & 20 & 2.5 \\
400 & 50 & 35.8 & 295.9 & 36.7 & 14.3 & 14.1 & 0.986 & 20 & 2.5 \\
& & & & & & & & & \\
450 & 10 & 40.5 & 331.6 & 40.5 & 18.34 & 17.22 & 0.939 & 20 & 2.5 \\
450 & 50 & 40.9 & 335.4 & 41.4 & 18.53 & 18.09 & 0.976 & 20 & 2.5 \\
450 & 70 & 45.5 & 337.1 & 42.5 & 18.66 & 18.36 & 0.984 & 20 & 2.5 \\
\hline
\end{tabular}

Table 4 displays the test results when the unit was operated with a $325-\mathrm{Vdc}$ link for short-duration testing with $70^{\circ} \mathrm{C}$ coolant and a flow rate of $2.5 \mathrm{gpm}$. The unit was operated at output frequencies of 10 , 20,50,75, and $100 \mathrm{~Hz}$ with the same requested dc link currents of 20,40, 60, 80, and $100 \mathrm{~A}$ during the test. The Semikron inverter demonstrated efficiencies near or above the target efficiency (i.e., 0.97) when operated at a reasonable load. In general, the higher efficiencies were demonstrated when the unit was operated with an output frequency of $20 \mathrm{~Hz}$. Figure 4 plots efficiency vs output power for the inverter based on the test results in Table 4.

The inverter was tested at continuous output power levels (i.e., $30 \mathrm{~kW}$ ) for $60 \mathrm{~min}$ with $70^{\circ} \mathrm{C}$ coolant at a flow rate of $2.5 \mathrm{gpm}$. Figure 5 shows the test data from the power meter and the current waveforms for the inverter. The inverter was operating with an efficiency of 0.972 at the end of the 60-min test. Phase A, phase B, and the dc link currents are displayed in the Figure 5 plot. The two-wattmeter method was used to measure the inverter's output per the Blondel theorem. Element 1 measured the input; elements 2 and 3 measured the output of phases A and B. Element 4 was not used in the test. Sum A and $\mathrm{B}$ are the summations of the dc input and the output of the inverter, respectively. Table 5 displays test data from the continuous power test.

The inverter was operated at power levels greater than the continuous power level for short durations with $70^{\circ} \mathrm{C}$ coolant. The inverter demonstrated a peak power capability of $57.7 \mathrm{~kW}$ with an efficiency of 0.976 when operated with a dc link of $400 \mathrm{~V}$. The inverter produced $38.6 \mathrm{~kW}$ of output power when operated with a $325-\mathrm{Vdc}$ link and $41.2 \mathrm{~kW}$ when operated with a $335 \mathrm{Vdc}$ link. Figure 6 shows the power meter readings and the current waveforms at peak power conditions. The inverter's input and output power was $59.17 \mathrm{~kW}$ and $57.74 \mathrm{~kW}$, respectively, which provides an efficiency of 0.976 . Table 6 summarizes the test results for short-duration testing at power levels greater than $30 \mathrm{~kW}$. 
Table 4. Short-duration testing of Semikron inverter with 325-Vdc link

\begin{tabular}{cccccccccc}
\hline dc link & Freq & Idc & Vac & Iac & Pin & Pout & Effic. & Cool. & Flow \\
\hline$(\mathbf{V d c})$ & $(\mathbf{H z})$ & $(\mathbf{A})$ & $\left(\mathbf{V}_{\mathbf{L L}}\right)$ & $\left(\mathbf{A}_{\mathbf{r m s}}\right)$ & $(\mathbf{k W})$ & $(\mathbf{k W})$ & & $(\mathbf{C})$ & $(\mathbf{g p m})$ \\
325 & 10 & 5.4 & 108.2 & 27.0 & 1.8 & 1.6 & 0.892 & 70 & 2.5 \\
325 & 10 & 18.6 & 150.3 & 45.5 & 6.0 & 5.7 & 0.944 & 70 & 2.5 \\
325 & 10 & 40.1 & 182.4 & 65.2 & 13.0 & 12.4 & 0.957 & 70 & 2.5 \\
325 & 10 & 69.4 & 209.6 & 85.3 & 22.5 & 21.8 & 0.971 & 70 & 2.5 \\
325 & 10 & 104.4 & 232.6 & 104.0 & 33.7 & 32.8 & 0.973 & 70 & 2.5 \\
& & & & & & & & & \\
325 & 20 & 5.5 & 107.9 & 27.7 & 1.8 & 1.6 & 0.898 & 70 & 2.5 \\
325 & 20 & 18.7 & 150.3 & 45.9 & 6.0 & 5.8 & 0.957 & 70 & 2.5 \\
325 & 20 & 39.37 & 183.8 & 65.1 & 12.81 & 12.4 & 0.968 & 70 & 2.5 \\
325 & 20 & 69.7 & 210.1 & 85.7 & 22.4 & 22.0 & 0.983 & 70 & 2.5 \\
325 & 20 & 104.7 & 232.9 & 104.1 & 33.7 & 32.9 & 0.976 & 70 & 2.5 \\
& & & & & & & & & \\
325 & 50 & 5.1 & 110.9 & 26.9 & 1.6 & 1.5 & 0.909 & 70 & 2.5 \\
325 & 50 & 18.2 & 155.6 & 45.4 & 5.9 & 5.6 & 0.956 & 70 & 2.5 \\
325 & 50 & 39.8 & 188.9 & 65.3 & 12.8 & 12.3 & 0.964 & 70 & 2.5 \\
325 & 50 & 69.3 & 217.6 & 85.3 & 22.3 & 21.6 & 0.969 & 70 & 2.5 \\
325 & 50 & 105.9 & 242.2 & 105.5 & 34.0 & 33.2 & 0.976 & 70 & 2.5 \\
& & & & & & & & & \\
325 & 75 & 5.2 & 110.8 & 27.2 & 1.7 & 1.5 & 0.873 & 70 & 2.5 \\
325 & 75 & 18.3 & 163.3 & 45.5 & 5.9 & 5.6 & 0.959 & 70 & 2.5 \\
325 & 75 & 39.6 & 198.1 & 65.3 & 12.7 & 12.3 & 0.972 & 70 & 2.5 \\
325 & 75 & 68.9 & 228.5 & 85.4 & 22.1 & 21.4 & 0.970 & 70 & 2.5 \\
325 & 75 & 105.1 & 253.5 & 104.7 & 33.7 & 32.8 & 0.973 & 70 & 2.5 \\
325 & 100 & 5.0 & 122.5 & 26.4 & 1.6 & 1.4 & 0.894 & 70 & 2.5 \\
325 & 100 & 18.3 & 172.2 & 45.1 & 5.9 & 5.6 & 0.949 & 70 & 2.5 \\
325 & 100 & 39.4 & 209.8 & 64.8 & 12.6 & 12.3 & 0.969 & 70 & 2.5 \\
325 & 100 & 68.3 & 241.0 & 84.5 & 21.9 & 21.3 & 0.974 & 70 & 2.5 \\
325 & 100 & 84.2 & 257.2 & 94.0 & 27.4 & 26.7 & 0.974 & 70 & 2.5 \\
\hline & & & & & & & & &
\end{tabular}




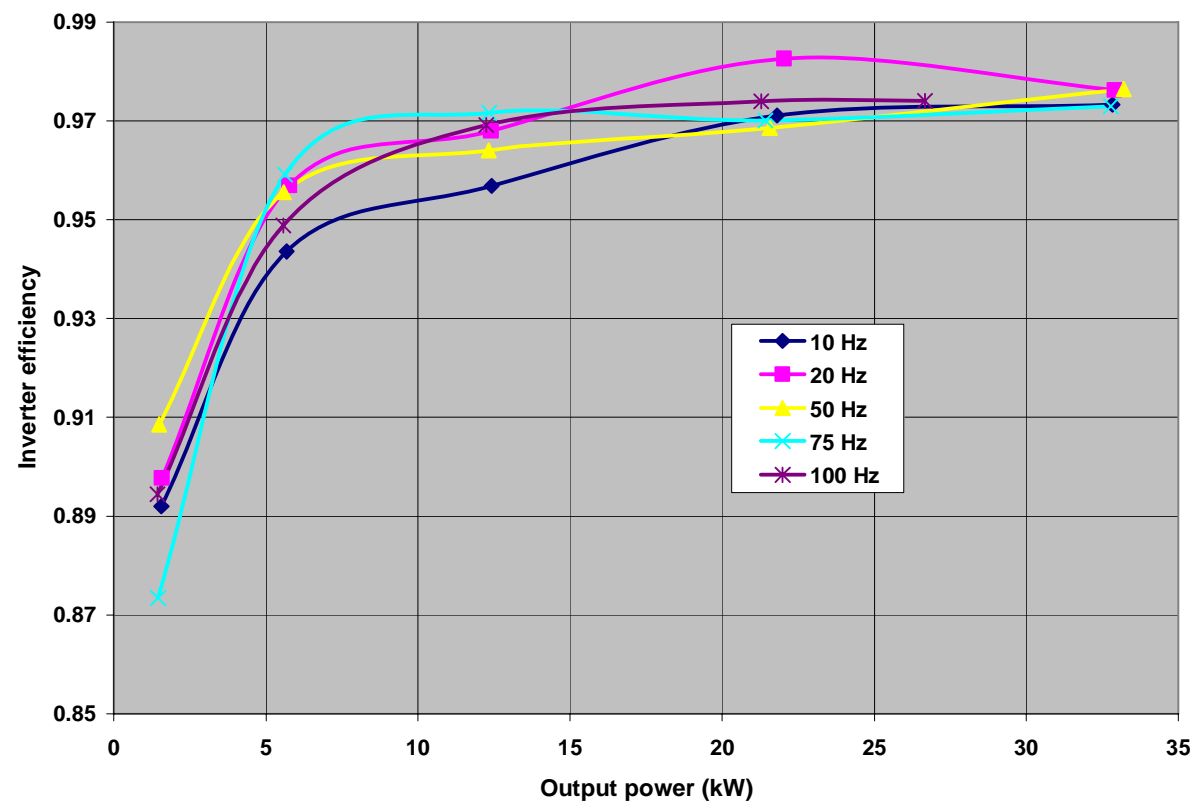

Figure 4. Inverter efficiency vs output power with $70^{\circ} \mathrm{C}$ coolant.

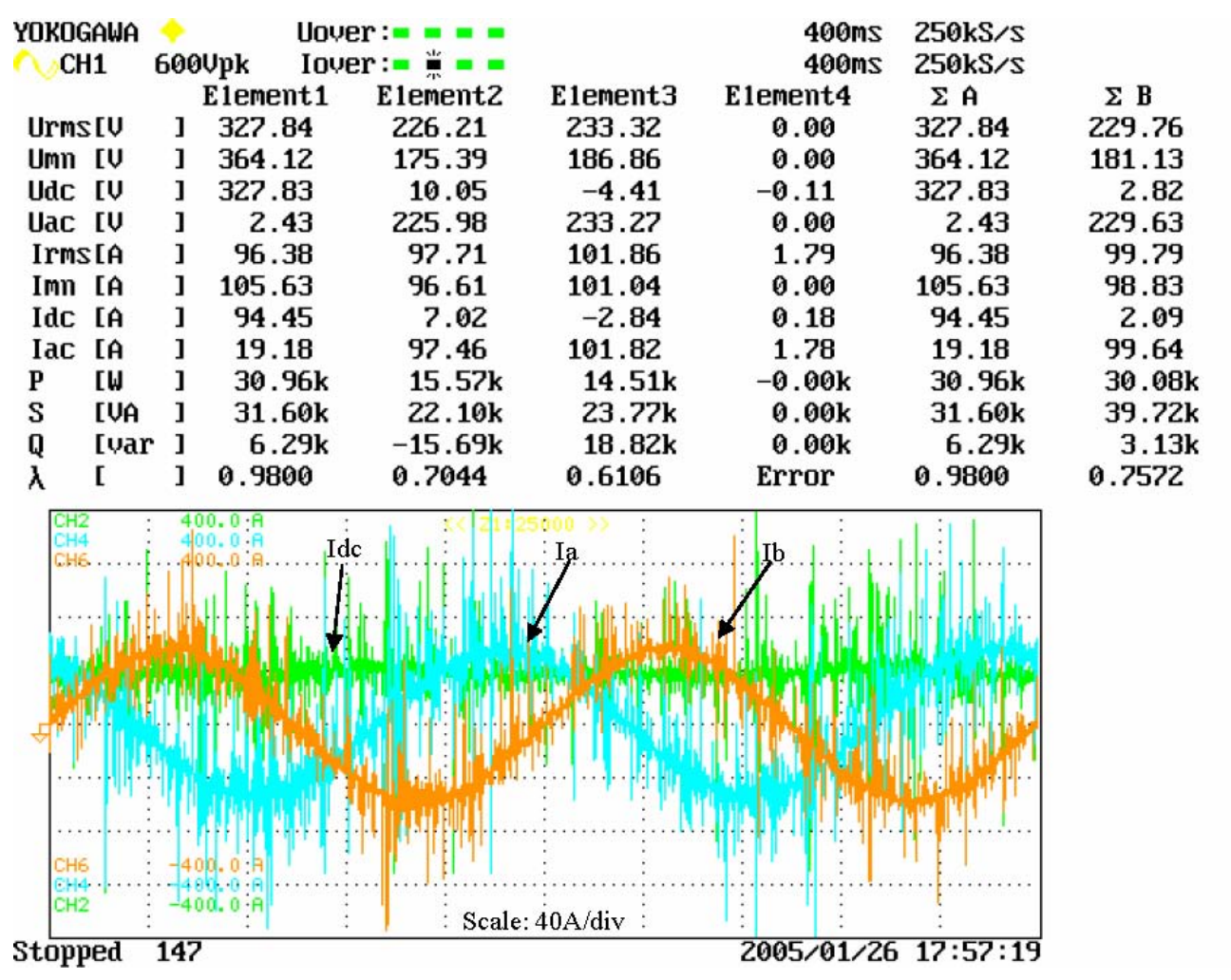

Figure 5. Power meter readings and current waveforms at end of continuous power test with $70^{\circ} \mathrm{C}$ coolant. 
Table 5. Continuous power testing of Semikron inverter with 325-Vdc link

\begin{tabular}{ccccccccccc}
\hline dc link & Freq & Idc & Vac & Iac & Pin & Pout & Effic. & Cool. & Flow & Time \\
\hline$(\mathbf{V d c})$ & $(\mathbf{H z})$ & $(\mathbf{A})$ & $\left(\mathbf{V}_{\mathbf{L L}}\right)$ & $\left(\mathbf{A}_{\mathbf{r m s}}\right)$ & $(\mathbf{k W})$ & $(\mathbf{k W})$ & & $(\mathbf{C})$ & $(\mathbf{g p m})$ & $(\mathbf{m})$ \\
325 & 20 & 94.99 & 229.6 & 99.4 & 30.9 & 30.1 & 0.975 & 70 & 2.5 & 5 \\
325 & 20 & 95.79 & 228.8 & 99.9 & 31.0 & 30.2 & 0.976 & 70 & 2.5 & 30 \\
325 & 20 & 94.47 & 229.8 & 99.5 & 31.0 & 30.0 & 0.967 & 70 & 2.5 & 45 \\
325 & 20 & 94.45 & 229.8 & 99.8 & 31.0 & 30.1 & 0.972 & 70 & 2.5 & 60 \\
\hline
\end{tabular}

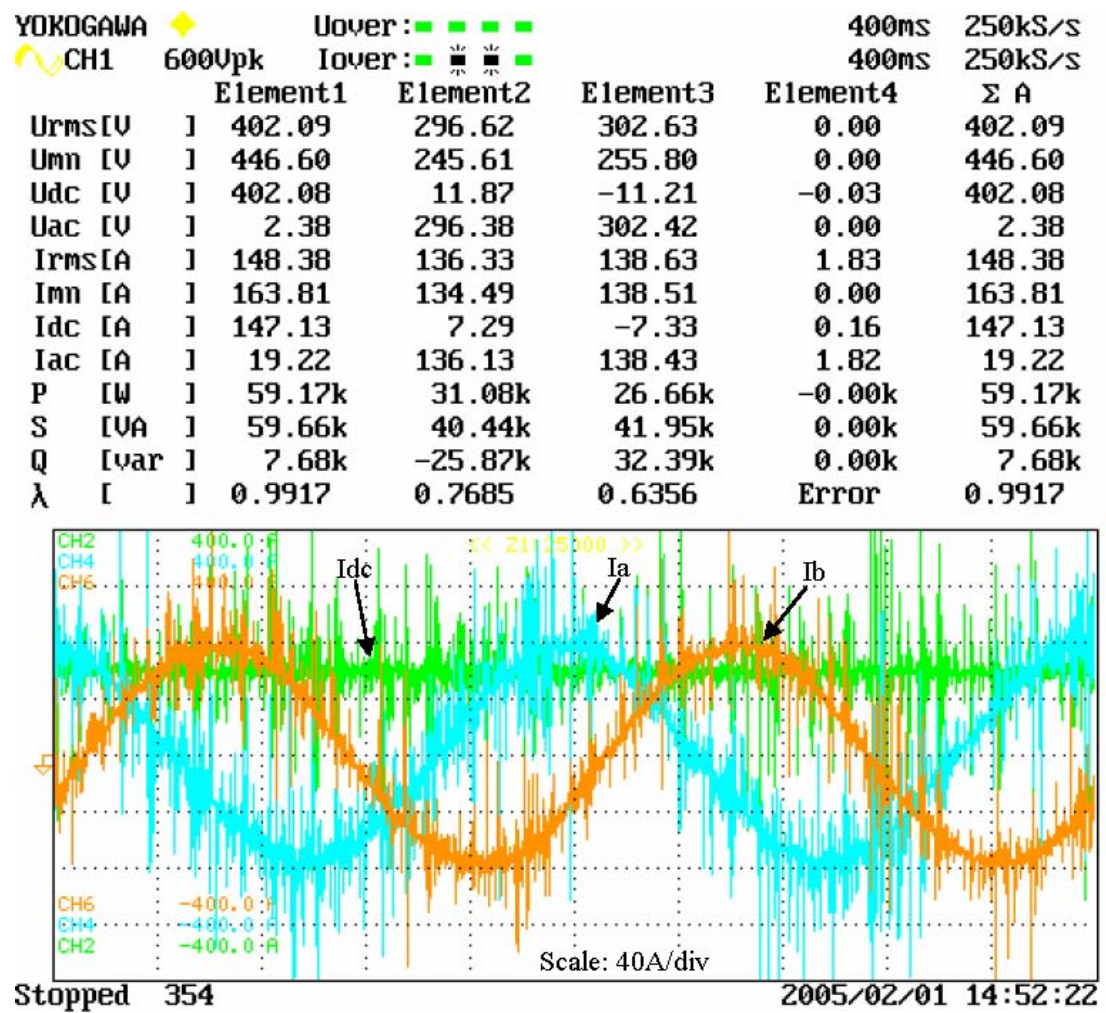

Figure 6. Power meter readings and current waveforms at end of peak power test with $70^{\circ} \mathrm{C}$ coolant.

Table 6. Testing of Semikron inverter at greater than continuous power levels

\begin{tabular}{cccccccccc}
\hline dc link & Freq & Idc & Vac & Iac & Pin & Pout & Effic. & Cool. & Flow \\
\hline$(\mathbf{V d c})$ & $(\mathbf{H z})$ & $(\mathbf{A})$ & $\left(\mathbf{V}_{\mathbf{L L}}\right)$ & $\left(\mathbf{A}_{\text {rms }}\right)$ & $(\mathbf{k W})$ & $(\mathbf{k W})$ & & $(\mathbf{C})$ & $(\mathbf{g p m})$ \\
325 & 20 & 118.7 & 242.1 & 111.2 & 38.6 & 37.7 & 0.977 & 70 & 2.5 \\
335 & 30 & 122.5 & 122.7 & 114.9 & 41.2 & 40.0 & 0.970 & 70 & 2.5 \\
400 & 20 & 147.1 & 299.6 & 137.5 & 59.2 & 57.7 & 0.976 & 70 & 2.5 \\
\hline
\end{tabular}




\section{Dynamometer testing}

The inverter was tested while driving a Solectria motor in the 100-HP dynamometer cell at ORNL. The Solectria motor is a four-pole induction motor with a base speed of $2500 \mathrm{rpm}$. Dynamometer testing was performed with a dc link voltage of $325 \mathrm{~V}$ and with the inverter being supplied with $70^{\circ} \mathrm{C}$ coolant at a flow rate of $2.5 \mathrm{gpm}$. The inverter supplied electrical energy to drive the induction motor from 750 to $2500 \mathrm{rpm}$ with a torque load of 100,150 , and $200 \mathrm{Nm}$.

Figure 7 plots the inverter efficiency vs motor speed during the test. The highest efficiency (0.97) was obtained at $2500 \mathrm{rpm}$ with a load of $150 \mathrm{Nm}$ on the Solectria motor. Table 7 presents the test data for the tests at 100, 150, and $200 \mathrm{Nm}$. The higher inverter efficiency was obtained near the base speed on the motor at the higher torque loads. The inverter meets the efficiency goal at a speed of $2500 \mathrm{rpm}$.

The inverter was tested at continuous power $(30-\mathrm{kW})$ conditions with the load motor speed ranging from 1000 to $2400 \mathrm{rpm}$. Figure 8 shows the tests results at seven different motor speeds with the inverter supplying $30 \mathrm{~kW}$ of electrical power. The inverter had an efficiency of 0.97 at $2400 \mathrm{rpm}$ with an output of $30 \mathrm{~kW}$. Figure 9 shows the power meter readings and current waveforms during the continuous power test on the dyne.

Table 8 summarizes weight and volume measurements, power density measurements, and goals for the Semikron AIPM. The unit has a peak power requirement of $55 \mathrm{~kW}$. At that value the unit had a peak specific power density of 7.1 peak $\mathrm{kW} / \mathrm{kg}$, exceeding the goal of 5 peak $\mathrm{kW} / \mathrm{kg}$. The unit had a peak volumetric power density of 8 peak $\mathrm{kW} / \mathrm{L}$, which failed to meet the goal of 12 peak $\mathrm{kW} / \mathrm{L}$.

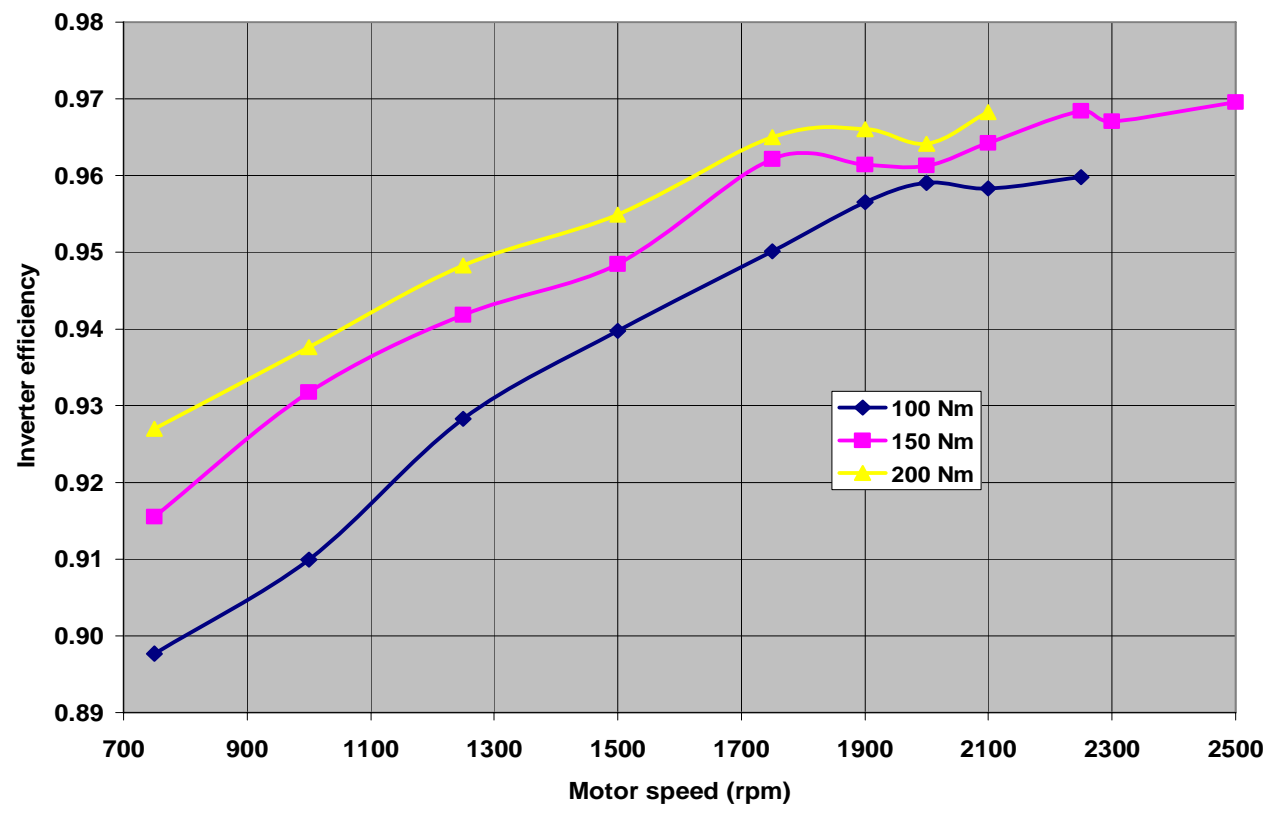

Figure 7. Inverter efficiency vs motor speed with $70^{\circ} \mathrm{C}$ coolant. 
Table 7. Dynamometer testing of Semikron inverter-speed vs inverter efficiency

\begin{tabular}{cccccccccc}
\hline dc link & Speed & Torq & Idc & Vac & Iac & Pin & Pout & Mech & AIPM \\
\hline$(\mathbf{V d c})$ & $(\mathbf{r p m})$ & $(\mathbf{N m})$ & $(\mathbf{A})$ & $\left(\mathbf{V}_{\mathbf{L L}}\right)$ & $\left(\mathbf{A}_{\mathbf{r m s}}\right)$ & $(\mathbf{k W})$ & $(\mathbf{k W})$ & $(\mathbf{k W})$ & $\mathbf{E f f i c}$ \\
325 & 750 & 100 & 34.3 & 145.7 & 155.0 & 11.2 & 10.1 & 7.8 & 0.898 \\
325 & 1000 & 100 & 43.7 & 167.9 & 166.6 & 14.3 & 13.0 & 10.4 & 0.910 \\
325 & 1250 & 100 & 49.5 & 104.4 & 157.2 & 16.3 & 15.1 & 13.1 & 0.928 \\
325 & 1500 & 100 & 58.4 & 202.4 & 162.2 & 19.3 & 18.1 & 15.6 & 0.940 \\
325 & 1750 & 100 & 65.2 & 211.7 & 134.5 & 21.5 & 20.4 & 18.3 & 0.950 \\
325 & 1900 & 100 & 70.6 & 222.5 & 140.5 & 23.2 & 22.2 & 19.9 & 0.957 \\
325 & 2000 & 100 & 72.7 & 222.6 & 130.0 & 23.9 & 23.0 & 20.9 & 0.959 \\
325 & 2100 & 100 & 78.0 & 235.3 & 144.3 & 25.7 & 24.6 & 21.9 & 0.958 \\
325 & 2250 & 100 & 82.5 & 241.3 & 142.7 & 27.1 & 26.0 & 23.5 & 0.960 \\
& & & & & & & & & \\
325 & 750 & 150 & 49.5 & 147.5 & 178.0 & 16.2 & 14.9 & 11.8 & 0.916 \\
325 & 1000 & 150 & 62.0 & 169.8 & 189.1 & 20.7 & 19.2 & 15.7 & 0.932 \\
325 & 1250 & 150 & 72.1 & 184.9 & 180.5 & 23.7 & 22.4 & 19.6 & 0.942 \\
325 & 1500 & 150 & 85.5 & 203.0 & 184.4 & 28.2 & 26.7 & 23.5 & 0.948 \\
325 & 1750 & 150 & 97.2 & 208.7 & 161.3 & 32.0 & 30.8 & 27.4 & 0.962 \\
325 & 1900 & 150 & 105.7 & 223.7 & 169.6 & 34.7 & 33.4 & 31.4 & 0.961 \\
325 & 2000 & 150 & 109.2 & 211.1 & 165.6 & 35.9 & 34.5 & 31.4 & 0.961 \\
325 & 2100 & 150 & 115.9 & 234.3 & 170.1 & 38.1 & 36.7 & 33.0 & 0.964 \\
325 & 2250 & 150 & 121.5 & 233.7 & 162.4 & 39.9 & 38.6 & 35.2 & 0.968 \\
325 & 2300 & 150 & 127.2 & 251.2 & 190.3 & 41.9 & 40.5 & 36.1 & 0.967 \\
325 & 2500 & 150 & 140.5 & 232.1 & 178.1 & 46.1 & 44.7 & 40.0 & 0.970 \\
& & & & & & & & & \\
325 & 750 & 200 & 19.2 & 149.3 & 206.5 & 21.8 & 20.2 & 15.7 & 0.927 \\
325 & 1000 & 200 & 83.7 & 170.8 & 215.8 & 27.4 & 25.7 & 20.9 & 0.938 \\
325 & 1250 & 200 & 96.5 & 185.8 & 209.9 & 31.7 & 30.1 & 26.1 & 0.948 \\
325 & 1500 & 200 & 114.0 & 204.0 & 215.6 & 37.5 & 35.8 & 31.4 & 0.955 \\
325 & 1750 & 200 & 132.2 & 212.9 & 204.1 & 43.4 & 41.9 & 36.7 & 0.965 \\
325 & 1900 & 200 & 141.9 & 221.9 & 205.0 & 46.6 & 45.0 & 39.8 & 0.966 \\
325 & 2000 & 200 & 147.8 & 217.2 & 210.2 & 48.5 & 46.8 & 42.0 & 0.964 \\
325 & 2100 & 200 & 155.9 & 234.0 & 206.0 & 51.1 & 49.5 & 43.9 & 0.968 \\
\hline & & & & & & & & & \\
& & & & & & & \\
& & & & & \\
325 &
\end{tabular}




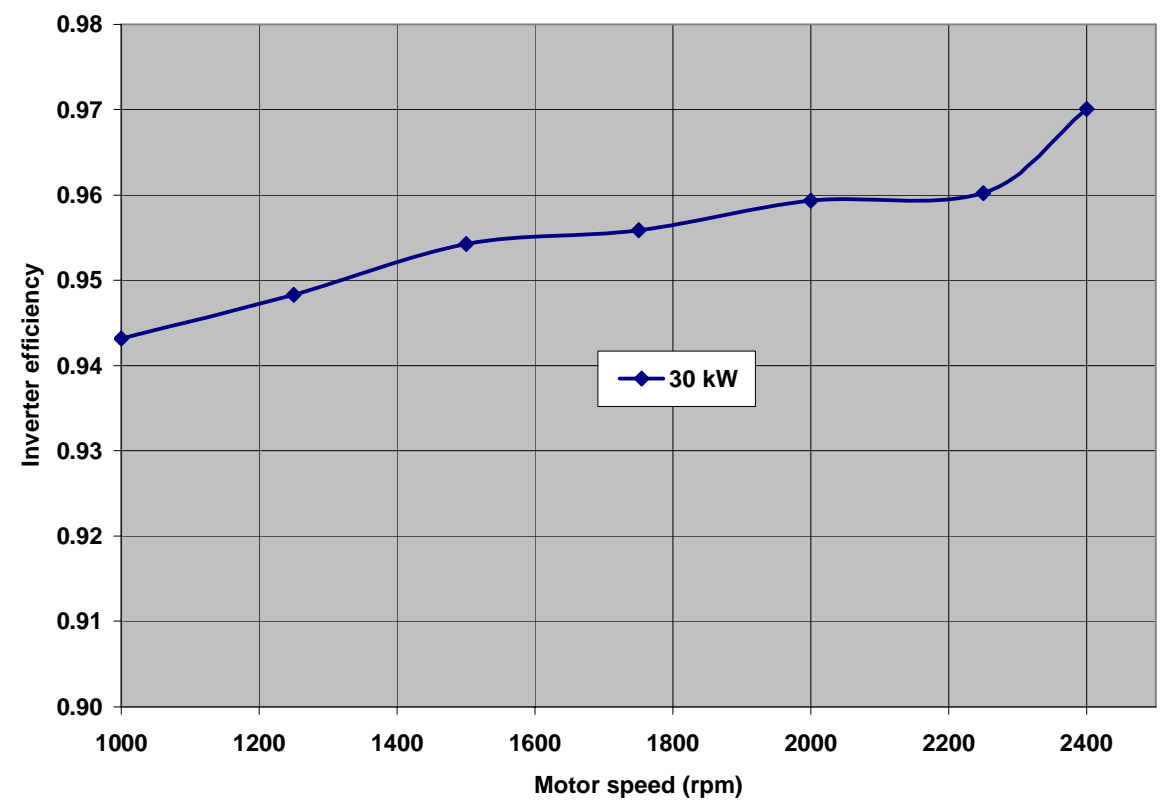

Figure 8. Semikron inverter efficiency vs speed at continuous power with $70^{\circ} \mathrm{C}$ coolant.

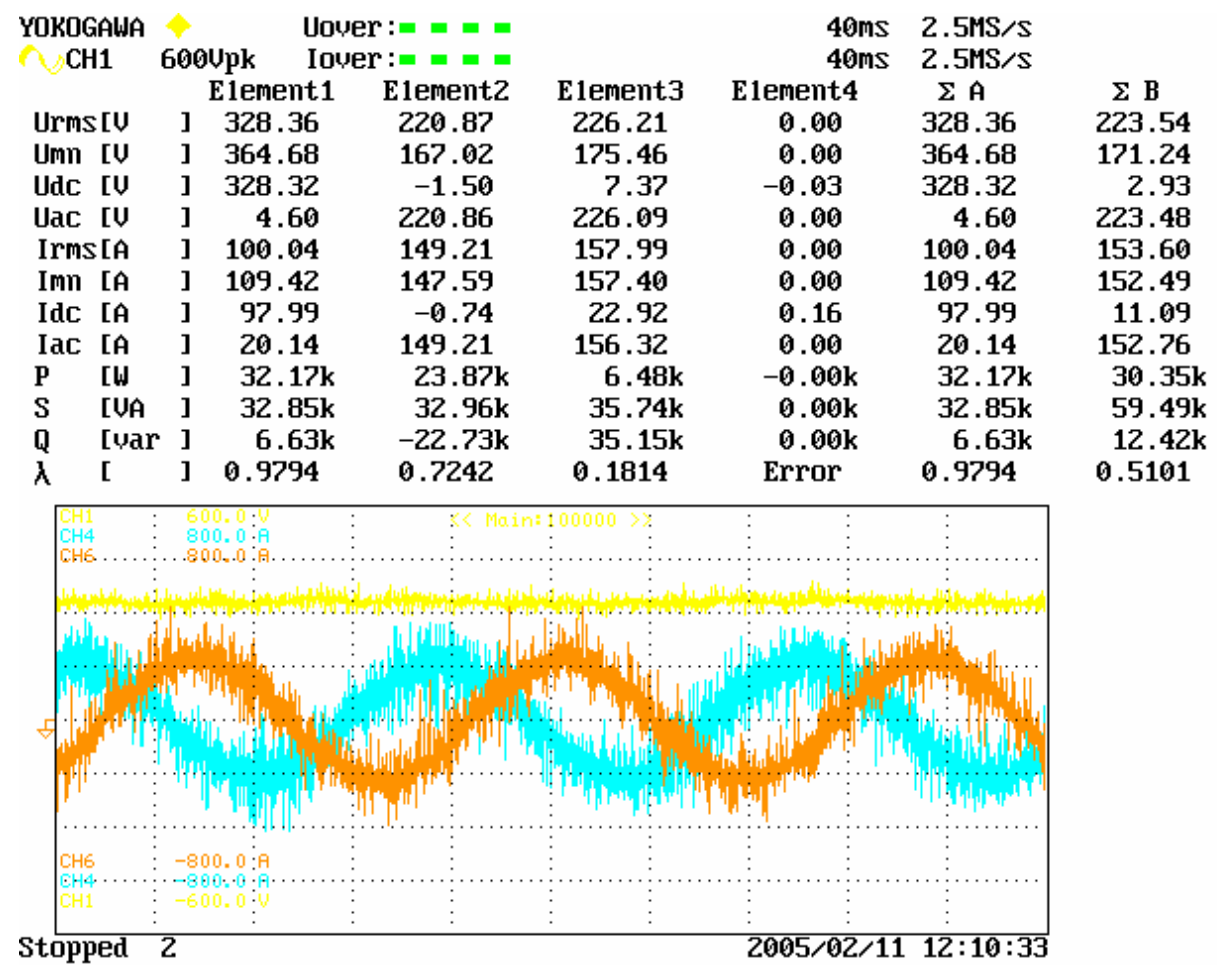

Figure 9. Power meter readings and current waveforms during continuous power test on dyne with $70^{\circ} \mathrm{C}$ coolant. 
Table 8. Specific power density summary

\begin{tabular}{lcc}
\hline Characteristic & Semikron measurement & FreedomCAR Goal \\
\hline Weight (kg) & 7.7 & NA \\
Volume (L) & 6.9 & NA \\
Peak power (kW) & 55 & 55 \\
$\begin{array}{l}\text { Peak specific power density } \\
\text { (peak kW/kg) }\end{array}$ & 7.1 & 5 \\
$\begin{array}{l}\text { Peak volumetric power density } \\
\text { (peak kW/L) }\end{array}$ & 8 & 12 \\
\hline
\end{tabular}

\section{Conclusion}

The AIPM unit demonstrated the ability to operate at continuous and peak power levels with an efficiency of 0.97 . The unit had a specific power density of $7.1 \mathrm{~kW} / \mathrm{kg}$, exceeding the goal of $5 \mathrm{~kW} / \mathrm{kg}$, based upon the FreedomCAR-specified $55-\mathrm{kW}$ peak power rating. The unit had a volumetric power density of 8 peak kW/L, failing to meet the FreedomCAR goal of $12 \mathrm{~kW} / \mathrm{L}$.

The Semikron AIPM has a capability of operation at a peak power rating of $160 \mathrm{~kW}$ with coolant supplied at $25^{\circ} \mathrm{C}$. At that rating the unit has a volumetric power density of 23 peak $\mathrm{kW} / \mathrm{L}$ and a power density of 21 peak $\mathrm{kW} / \mathrm{kg}$.

\section{Future Direction}

Testing of the Semikron unit in January 2005 completes this task. Other inverter development and/or test activity will continue as needed.

\section{Publications}

Testing of the Semikron Validation AIPM Unit at ORNL_January 2005, ORNL/TM-2005/44. 
ORNL/TM-2005/264

\section{Distribution}

\section{Internal}

1. D. J. Adams

2. C. W. Ayers

3. J. M. Bailey

4. J. B. Campbell

5. C. L. Coomer

6. K. P. Gambrell

7. J. S. Hsu

8. L. D. Marlino

9. J. W. McKeever

10. S. C. Nelson, Jr.
11. M. Olszewski
12. P. J. Olszewski
13. P. J. Otaduy
14. B. Ozpineci
15. L. E. Seiber
16. R. H. Staunton
17. G. J. Su
18. L. M. Tolbert
19. R. H. Wiles
20. Laboratory Records

\section{External}

21. T. Q. Duong, U.S. Department of Energy, EE-2G/Forrestal Building, 1000 Independence Avenue, S.W., Washington, D.C. 20585.

22. R. R. Fessler, BIZTEK Consulting, Inc., 820 Roslyn Place, Evanston, Illinois 60201-1724.

23. K. Fiegenschuh, Ford Motor Company, Scientific Research Laboratory, 2101 Village Road, MD2247, Dearborn, Michigan 48121.

24. V. Garg, Ford Motor Company, 15050 Commerce Drive, North, Dearborn, Michigan 48120-1261.

25. E. Jih, Ford Motor Company, Scientific Research Laboratory, 2101 Village Road, MD-1170, Rm. 2331, Dearborn, Michigan 48121.

26. A. Lee, Daimler Chrysler, CIMS 484-08-06, 800 Chrysler Drive, Auburn Hills, Michigan 483262757.

27. F. Liang, Ford Motor Company, Scientific Research Laboratory, 2101 Village Road, MD1170, Rm. 2331/SRL, Dearborn, Michigan 48121.

28. M. W. Lloyd, Energetics, Inc., 7164 Columbia Gateway Drive, Columbia, Maryland 21046.

29. M. Mehall, Ford Motor Company, Scientific Research Laboratory, 2101 Village Road, MD-2247, Rm. 3317, Dearborn, Michigan 48124-2053.

30. N. Olds, USCAR, nolds@uscar.org

31. J. Rogers, Chemical and Environmental Sciences Laboratory, GM R\&D Center, 30500 Mound Road, Warren, Michigan 48090-9055.

32. S. A. Rogers, U.S. Department of Energy, EE-2G/Forrestal Building, 1000 Independence Avenue, S.W., Washington, D.C. 20585.

33. G. S. Smith, General Motors Advanced Technology Center, 3050 Lomita Boulevard, Torrance, California 90505.

34. E. J. Wall, U.S. Department of Energy, EE-2G/Forrestal Building, 1000 Independence Avenue, S.W., Washington, D.C. 20585.

35. B. Welchko, General Motors Advanced Technology Center, 3050 Lomita Boulevard, Torrance, California 90505.

36. P. G. Yoshida, U.S. Department of Energy, EE-2G/Forrestal Building, 1000 Independence Avenue, S.W., Washington, D.C. 20585 